Uma sequência exata relacionada a uma extensão de anéis e uma representação parcial

\author{
Josefa Itailma da Rocha
}

TESE APRESENTADA

Instituto de MATEMÁtica E Estatística

Universidade DE SÃo PaUlo

PARA
OBTENÇÁO DO TÍTULO

DOUTOR EM CIÊNCIAS

Programa: Matemática

Orientador: Prof. Dr. Mikhailo Dokuchaev

São Paulo, dezembro de 2017 


\section{Uma sequência exata relacionada a uma extensão de anéis e uma representação parcial}

Esta versão da tese contém as correções e alterações sugeridas pela Comissão Julgadora durante a defesa da versão original do trabalho, realizada em 27/02/2018. Uma cópia da versão original está disponível no Instituto de Matemática e Estatística da Universidade de São Paulo.

Comissão Julgadora:

- Prof ${ }^{\mathrm{a}}$. Dr Mikhailo Dokuchaev (orientador) - IME-USP

- Prof. Dr. Javier Sánchez Serdá - IME-USP

- Prof. Dr. Fernando Raul Abadie Vicens - Universidad de la República

- Prof. Dr. Héctor Edonis Pinedo Tapia - Universidad Industrial de Santander

- Prof. Dr. Mykola Khrypchenko - UFSC 


\section{Resumo}

ROCHA, J. I. Uma sequência exata relacionada a uma extensão de anéis e uma representação parcial. 2017. $114 \mathrm{f}$. Tese (Doutorado) - Instituto de Matemática e Estatística, Universidade de São Paulo, São Paulo, 2017.

Para uma extensão de Galois de anéis comutativos, Chase-Harrison-Rosenberg construíram uma sequência exata de sete termos que envolve o grupo de Picard, o grupo de Brauer relativo e grupos de cohomologias. Essa sequência é vista como uma generalização de dois fatos importantes da teoria galoisiana de corpos, a saber, o Teorema 90 de Hilbert e o isomorfismo de grupo de Brauer relativo com o segundo grupo de cohomologia. A sequência foi generalizada por Miyashita para o contexto de anéis não comutativos com unidade. Mais tarde, El Kaoutit e Gomez-Torrencillas generalizaram o resultado de Miyashita para uma extensão de anéis não comutativos e não unitais, apenas com um conjunto de unidades locais. A sequência de Chase-Harrison-Rosenberg também foi considerada para ações parciais por Dokuchaev, Paques e Pinedo, que construíram uma versão para uma extensão de Galois parcial de anéis comutativos. Nesta tese, elaboramos uma versão da sequência no contexto de ações parciais para uma extensão de anéis não comutativos com unidade. A sequência apresentada aqui generaliza a sequência dada por Miyashita.

Palavras-chave: ações parciais, representação parcial, produto cruzado generalizado parcial. 


\section{Abstract}

ROCHA. J.I. An exact sequence related to an extension of rings and a partial representation . 2017. 114 f. Tese (Doutorado) - Instituto de Matemática e Estatística, Universidade de São Paulo, São Paulo, 2010.

For a Galois extension of commutative rings, Chase-Harrison-Rosenberg constructed a seven terms exact sequence which involves the Picard group, the relative Brauer group and cohomology groups. The sequence can be viewed as a generalization of two important facts of Galois theory of fields: the Hilbert 90 Theorem and the isomorphism of the relative Brauer group with the second cohomology group. The sequence was generalized by Miyashita for the context of non-commutative unital rings. Later, El Kaoutit and Gomez-Torrencillas extended the result of Miyashita for an extension of non-unital non-commutative rings with local units. The Chase-Harrison-Rosenberg sequence was also considered for partial actions by Dokuchaev, Paques e Pinedo, who constructed a version for a partial Galois extension of commutative rings. In this thesis, we elaborate a vesrion of the sequence in the context of partial actions for an extension of non-commutative unital rings. Our sequence generalizes the sequence given by Miyashita.

Keywords: partial actions, partial representations, partial generalized crossed products 


\section{Sumário}

1 Introdução $r$

2 Conceitos Básicos $\quad 5$

2.1 Módulos projetivos e o grupo de Picard . . . . . . . . . . . . . . . 5

2.2 O semigrupo de Picard generalizado . . . . . . . . . . . . . . . . . . . . . 20

$2.3 R$-bimódulos similares . . . . . . . . . . . . . . . . . . . . . . 22

$2.4 \quad \mathrm{O}$ grupo $\mathcal{P}(S / R) \ldots \ldots \ldots \ldots \ldots \ldots \ldots \ldots \ldots$

2.5 Uma sequência exata auxiliar . . . . . . . . . . . . . . . . . . . . . . . 28

3 Fundamentos sobre ações e representações parciais 31

3.1 Ações parciais, representações parciais e cohomologia parcial. . . . . . . . . . . . . . . . . 31

3.2 Representação Parcial Unital . . . . . . . . . . . . . . . . . . . . . . . 35

3.2.1 Ação parcial sobre $\mathcal{Z}$ e sobre $\mathbf{P i c S}_{\mathcal{Z}}(R) \ldots \ldots \ldots \ldots \ldots$

3.3 Produto cruzado generalizado parcial . . . . . . . . . . . . . . . . . . . . . . . 42

3.3.1 Representações parciais unitais em $\mathcal{S}_{R}(S) \ldots \ldots \ldots \ldots$. . . . . . . . . 56

$3.4 \mathrm{O}$ grupo $\mathcal{C}(\Theta / R) \ldots \ldots \ldots \ldots \ldots \ldots \ldots \ldots$

4 A sequência exata de sete termos $\quad 81$

4.1 A primeira sequência exata . . . . . . . . . . . . . . . . . . . 81

4.2 A segunda sequência exata . . . . . . . . . . . . . . . . . . . 86

$4.3 \mathrm{O}$ grupo $\mathcal{B}(\Theta / R)$ e a terceira sequência exata . . . . . . . . . . . . . . . . . . 104

4.4 O grupo $\overline{H^{1}}\left(G, \alpha^{*}, \mathbf{P i c S}_{0}(R)\right)$ e a quarta sequência exata . . . . . . . . . . . . . . . 104

4.5 A quinta sequência exata . . . . . . . . . . . . . . . . . . . . 107 


\section{Capítulo 1}

\section{Introdução}

O conceito de extensão Galoisiana de anéis comutativos foi introduzido em 1960 por M. Auslander e O. Goldman no artigo [1], no qual eles estabeleceram os fundamentos de extensões separáveis de anéis comutativos e definiram o grupo de Brauer de um anel comutativo. Em 1965, S. U. Chase, D.K. Harrison e A. Rosenberg desenvolveram a teoria de Galois de anéis comutativos em [2]. Usando cohomologia de Amitsur e sequências espectrais, Chase e Harrison construíram em [3] uma sequência exata de sete termos a qual foi aplicada em [2] para uma extensão de Galois $R \subseteq S$ de anéis comutativos com unidade e grupo de Galois $G$ obtendo a seguinte sequência exata:

$$
\begin{aligned}
1 \longrightarrow & H^{1}(G, \mathcal{U}(S)) \longrightarrow \operatorname{Pic}(R) \longrightarrow \operatorname{Pic}(S)^{G} \longrightarrow H^{2}(G, \mathcal{U}(S)) \longrightarrow B(S / R) \\
\longrightarrow & H^{1}(G, \operatorname{Pic}(S)) \longrightarrow H^{3}(G, \mathcal{U}(S)),
\end{aligned}
$$

onde $\mathcal{U}(S)$ denota o grupo dos inversíveis do anel $S$, Pic $(S)$ o grupo de Picard de $S$ e $B(S / R)$ o grupo de Brauer relativo de $S$ sobre $R$, ou seja, o grupo das $R$-álgebras de Azumaya que cindem por $S$. A sequência de Chase-Harrison-Rosenberg pode ser vista como uma generalização de dois fatos fundamentais de cohomologia galoisiana de corpos, que são o Teorema de Hilbert 90 e o Teorema do produto cruzado. O primeiro afirma que se $R \subseteq S$ é uma extensão de corpos de Galois, então $H^{1}(G, S)$ é trivial. Já o segundo diz que existe um isomorfismo entre o segundo grupo de cohomologias e o grupo de Brauer relativo da extensão $R \subseteq S$. A exatidão da sequência de acima implica nas seguintes generalizações para uma extensão de anéis comutativos com unidade:

Teorema 90 de Hilbert: Seja $R \subseteq S$ é uma extensão de Galois tal que $\mathbf{P i c}(R)=\{1\}$, então $H^{1}(G, \mathcal{U}(S))=\{1\}$.

Teorema do Produto cruzado: Seja $R \subseteq S$ é uma extensão de Galois com $\mathbf{P i c}(S)=\{1\}$, então $H^{2}(G, \mathcal{U}(S)) \simeq B(S / R)$.

Em 1968, no artigo [4], foi dada uma construção da sequência de Chase-Harrison-Rosenberg fazendo uso de produtos cruzados generalizados. A sequência de Chase-Harrison-Rosenberg também foi considerada no contexto de anéis não comutativos. Em 1973, motivado por [4] e [2], Y. Miyashita construiu no artigo [5] uma sequência exata de sete termos para uma extensão de anéis não comutativos com unidade que generaliza a sequência em [3]. Em 2012 a sequência de Miyashita foi generalizada por L. El. Koutit e J. Gómez-Torrecillas em [6] e [7]. Nestes trabalhos foi construída uma sequência exata de sete termos para uma extensão de anéis não comutativos e não unitais, apenas com um conjunto de unidades locais. 
Uma versão da sequência de Chase-Harrison-Rosenberg no contexto analítico/topológico foi elaborada por D. Crocker, I. Raeburn and D. Williams em [8].

O conceito de ações parciais surgiu na teoria de álgebra de operadores. No artigo de R. Exel [9] é introduzida a noção de uma ação parcial torcida de um grupo localmente compacto em uma $C^{*}$-álgebra e produto cruzado correspondente a essa ação e é estabelecida a associatividade desse produto cruzado. Em [10] é dada uma correspondência biunívoca entre as ações parciais de um grupo $G$ em conjunto e as ações globais de um semigrupo inverso construído a partir do grupo $G$ (ver [10]). Os trabalhos de Exel inspiraram diversos outros estudos em torno de ações parciais. Em particular, na tese de F. Abadie [11] (vide também o artigo [12]) foi iniciada a pesquisa do problema de globalização de uma ação parcial $\alpha$, i.e., a questão de existência de uma ação (global) $\beta$ de tal forma que $\alpha$ seja uma restrição de $\beta$. Uma tal ação $\beta$, com uma restrição natural que serve para garantir unicidade, é chamada de ação envolvente para $\alpha$. O estudo de ações parciais em um contexto puramente algébrico foi iniciado em 2005 por M. Dokuchaev e R. Exel em [13]. Nesse trabalho é associado a uma ação parcial um produto cruzado algébrico e obtido um resultado sobre sua associatividade. Também é dado um critério para existência de uma ação envolvente para uma ação parcial de um grupo sobre uma álgebra associativa com unidade (ver [13, Teorema 4.5]). Em 2007, M. Dokuchaev, A. Paques e M. Ferrero generalizaram em [14] a teoria de Galois de Chase, Harrison e Rosenberg [2]. Em [14] é assumido que a ação parcial admite uma ação envolvente. Nesse trabalho são dadas definições equivalentes para uma extensão de Galois de anéis comutativos, relação entre a extensão galoisina parcial de anéis comutativos e a extensão galoisiana envolvente e um Teorema fundamental. Posteriormente, em 2015, M. Dokuchaev e M. Khrypchenko desenvolveram em [15] a teoria de cohomologia parcial de grupos.

A sequência de Chase-Harrison-Rosenberg é considerada também no contexto de ações parciais. Em 2015, usando a teoria de Galois parcial e de cohomologia parcial, M. Dokuchaev, A. Paques e H. Pinedo construíram em [16] uma sequência de sete termos para uma extensão de Galois parcial de anéis comutativos. A exatidão da sequência construída em [16] foi provada em [17].

Neste trabalho, construímos uma versão no contexto de ações parciais da sequência exata de sete termos dada por Miyashita em [5] para uma extensão de anéis não comutativos com unidade.

O trabalho está organizado da seguinte forma. No Capítulo 2 encontram-se os conceitos básicos para o desenvolvimento do trabalho. Na Seção 2.1 demonstramos alguns resultados sobre módulos projetivos finitamente gerados e grupo de Picard sobre um anel não comutativo. Esses fatos são conhecidos e são usados em [18] e [5], por exemplo, mas suas demonstrações não são encontradas com tanta facilidade. Para anéis comutativos, esses resultados e suas demonstrações são encontradas por exemplo em [19], [20] e [21]. Na Seção 2.2 definimos o semigrupo de Picard de um anel não comutativo. Esse semigrupo foi definido em [16] para anéis comutativos. Nas seções seguintes são apresentados alguns resultados e definições de [5]. Algumas demonstrações também foram acrescentadas nessas seções, pois em [5] são omitidos vários detalhes que são importantes para o desenvolvimento do nosso trabalho.

No Capítulo 3 relembramos o conceito de ações parciais e representações parciais. Na Proposição 3.1.3 construímos uma ação parcial a partir de uma representação parcial de $G$ sobre um monoide $S$. Na Seção 3.2 definimos representação parcial unital de $G$ sobre $\mathbf{P i c S}(R)$ e mostramos suas propriedades. A partir de uma representação parcial unital construímos uma ação parcial sobre o centro do anel $R$ (Proposição 3.2.4), e uma ação parcial sobre $\mathbf{P i c S}(R)$ que é um caso particular da ação parcial construída na Proposição 3.1.3. Na Seção 3.3 definimos o produto cruzado generalizado parcial que é um anel associativo com unidade e pode ser visto como uma extensão do anel $R$. Na Observação 3.3.13 mostramos como construir um produto cruzado generalizado parcial a partir de uma extensão de anéis $R \subseteq S$ e uma representação parcial unital sobre o monoide dos $R$-subbmodulos de $S$ (ver Proposição 3.3.11). Na seção seguinte definimos o grupo $\mathcal{C}(\Theta / R)$, onde $\Theta: G \longrightarrow \operatorname{PicS}(R)$ é uma representação parcial unital fixada, que é formado por classes de isomorfismo de produtos cruzados generalizados parciais que satisfazem algumas condições (ver Proposição 3.4.1). Para encerrar a seção destacamos o subgrupo $\mathcal{C}_{0}(\Theta / R)$ e mostramos, no Teorema 3.4.3, o isomorfismo desse grupo com um segundo grupo de cohomologia parcial. 
No Capítulo 4 construímos a sequência exata de sete termos, no contexto parcial, que generaliza a sequência em [5, Teorema 2.12]. Usamos o produto cruzado generalizado parcial associado a uma extensão de anéis com mesma unidade e uma representação parcial unital para construir os morfismos e os grupos que aparecem na sequência. A exatidão da sequência é provada diretamente. 


\section{Capítulo 2}

\section{Conceitos Básicos}

Neste Capítulo vamos apresentar alguns resultados que serão usados no nosso trabalho. Em todo o texto $R$ denotará um anel (não comutativo) com unidade e $\mathcal{Z}$ o seu centro. Na primeira seção vamos demostrar alguns resultados sobre módulos projetivos finitamente gerados e grupo de Picard sobre um anel não comutativo. Esses resultados são conhecidos e, para anéis comutativos, suas demonstrações são facilmente encontradas nos textos básicos sobre módulos como por exemplo [19], [22], [20] e [21]. Já no caso de anéis não comutativos, essas demonstrações não são encontradas com tanta facilidade. Na Seção 2.2 definimos o semigrupo de Picard generalizado de um anel não comutativo $R$. Esse semigrupo foi introduzido em [16] para o contexto de anéis comutativos. Nas seções seguintes apresentamos algumas definições e resultados de [5] que serão usados ao longo do texto.

\subsection{Módulos projetivos e o grupo de Picard}

Seja $R$ um anel com unidade. Dizemos que um $R$-módulo à direita $M$ é unital se $m \cdot 1=m$, para todo $m \in M$. Neste caso, temos que $M R=M$ e que a aplicação $M \otimes_{R} R \longrightarrow M$, dada pela ação de $R$ em $M$, é um isomorfismo de $R$-módulos. Analogamente definimos $R$-módulos unitais à esquerda. Em todo o trabalho, estamos considerando apenas $R$-módulos unitais.

Definição 2.1.1. Um R-módulo à direita $P$ é chamado projetivo se dado qualquer diagrama

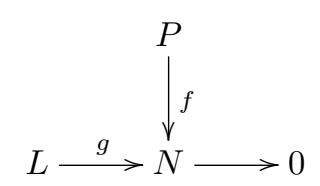

de R-módulos à direita, onde $g$ é sobrejetivo, existe um homomorfismo de $R$-módulos $f^{*}: P \longrightarrow L$ tal que o diagrama

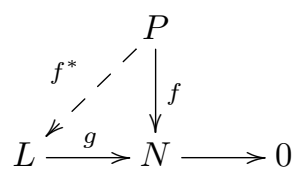

é comutativo.

Proposição 2.1.2. Seja $P$ um R-módulo à direita. São equivalentes:

(i) P é projetivo. 
(ii) Qualquer sequência exata $0 \longrightarrow M \longrightarrow N \longrightarrow P \longrightarrow 0$ cinde.

(iii) $P$ é somando direto de um R-módulo livre.

Demonstração. [22, Proposição 3.10].

Uma caracterização importante dos módulos projetivos é dada no Lema a seguir:

Lema 2.1.3 (Lema da Base dual). Seja $M$ um R-módulo à direita. Então, $M$ é projetivo se, e somente se, existem conjuntos $\left\{m_{i}\right\}_{i \in I} \subset M e\left\{f_{i}\right\}_{i \in I} \subset \operatorname{Hom}_{R}(M, R)$ tais que para qualquer $m \in M, f_{i}(m)=0$ a menos de um número finito de $i \in I$ e

$$
\sum_{i \in I} m_{i} f_{i}(m)=m
$$

A coleção $\left\{f_{i}, m_{i}\right\}_{i \in I}$ é chamada base dual de $M$.

Demonstração. [20, Lema 2.9].

No caso em que temos módulos finitamente gerados, o Lema da base tem a seguinte versão:

Corolário 2.1.4. Um R-módulo à direita $M$ é projetivo e finitamente gerado se, e somente se, existem $m_{i} \in M$ e $f_{i} \in \operatorname{Hom}_{R}(M, R) i=1,2, \ldots, n$, tais que para todo $m \in M$

$$
\sum_{i=1}^{n} m_{i} f_{i}(m)=m
$$

Se $R$ é comutativo, então todo $R$-módulo $M$ (à direita ou à esquerda) pode ser visto como um $R$ bimódulo (central) via a ação central: $r m=m r$, para todo $r \in R$ e $m \in M$. Para anéis não comutativos essa construção não é possível, ou seja, um $R$-módulo à direita nem sempre pode ser visto também como um $R$-módulo à esquerda. A partir daqui vamos trabalhar com $R$-bimódulos.

Sejam $M$ e $N$ dois $R$-bimódulos, então $\operatorname{Hom}\left(M_{R}, N_{R}\right)$ e $\operatorname{Hom}\left({ }_{R} M,_{R} N\right)$ são $R$-bimódulos via:

$$
(r \cdot f)(m)=r f(m) \text { e }(f \cdot r)(m)=f(r m), \quad f \in \operatorname{Hom}\left(M_{R}, N_{R}\right), m \in M, r \in R,
$$

e

$$
(r \cdot g)(m)=g(m r) \text { e }(g \cdot r)(m)=g(m) r, \quad g \in \operatorname{Hom}\left({ }_{R} M,_{R} N\right), m \in M, r \in R .
$$

Para um $R$-bimódulo $M$, vamos denotar ${ }^{*} M=\operatorname{Hom}\left(M_{R}, R_{R}\right)$ e $M^{*}=\operatorname{Hom}\left({ }_{R} M,_{R} R\right)$. Esses conjuntos são $R$-bimódulos com as estruturas definidas em (2.1) e (2.2).

Lema 2.1.5. Seja $P$ um R-bimódulo.

(i) Se P é R-módulo à direita projetivo e finitamente gerado com base dua $\left\{p_{i}, f_{i}\right\}_{i=1,2, \ldots, n}$, então ${ }^{*} P$ é $R$-módulo à esquerda projetivo e finitamente gerado com base dual $\left\{f_{i}, \Phi_{i}\right\}_{i=1,2, \ldots, n}$ onde

$$
\begin{aligned}
\Phi_{i}:{ }^{*} P & \longrightarrow R \\
f & \longmapsto f\left(p_{i}\right)
\end{aligned} .
$$

(ii) Se $P$ é $R$-módulo à esquerda projetivo e finitamente gerado com base dual $\left\{g_{i}, p_{i}\right\}_{i=1,2, \ldots, n}$, então $P^{*}$ é $R$-módulo à direita projetivo e finitamente gerado com base dual $\left\{g_{i}, \Psi_{i}\right\}$, onde

$$
\begin{aligned}
\Psi_{i}: P^{*} & \longrightarrow R \\
g & \longmapsto g\left(p_{i}\right)
\end{aligned}
$$


Demonstração. (i) Dado $r \in R$ temos

$$
\Phi_{i}(r \cdot f)=(r \cdot f)\left(p_{i}\right)=r f\left(p_{i}\right)=r \Phi_{i}(f) .
$$

Logo, $\Phi_{i} \in\left({ }^{*} P\right)^{*}=\operatorname{Hom}\left(R^{*} P,{ }_{R} R\right)$. Além disso, para todo $f \in{ }^{*} P$ temos

$$
\begin{aligned}
\sum_{i=1}^{n}\left(\Phi_{i}(f) \cdot f_{i}\right)(p) & =\sum_{i=1}^{n} \Phi_{i}(f) f_{i}(p)=\sum_{i=1}^{n} f\left(p_{i}\right) f_{i}(p) \\
& =\sum_{i=1}^{n} f\left(p_{i} f_{i}(p)\right)=f(p),
\end{aligned}
$$

para todo $p \in P$. Portanto, $\left\{\Phi_{i}, f_{i}\right\}_{i=1,2, \ldots, n}$ é uma base dual de ${ }^{*} P$ como $R$-módulo à esquerda.

(ii) Análogo ao item (i).

Lema 2.1.6. Seja $P$ um R-bimódulo. Então:

(i) Se P é um R-módulo à direita projetivo e finitamente gerado, então

$$
\begin{aligned}
\varphi: \quad P \otimes_{R}{ }^{*} P & \longrightarrow \\
p \otimes f & \longrightarrow\left(p^{\prime} \mapsto p f\left(P_{R}\right)\right),
\end{aligned}
$$

é um isomorfismo de R-bimódulos.

(ii) Se P é R-módulo à esquerda projetivo e finitamente gerado, então

$$
\begin{aligned}
\varphi^{\prime}: \quad P^{*} \otimes_{R} P & \longrightarrow \operatorname{End}\left({ }_{R} P\right) \\
f \otimes p & \longmapsto\left(p^{\prime} \mapsto f\left(p^{\prime}\right) p\right),
\end{aligned}
$$

é um isomorfismo de R-bimódulos.

Demonstração. (i) Claramente $\varphi$ está bem definida e é $R$-linear à esquerda. Por outro lado, dado $r \in R$, temos

$$
\varphi(p \otimes f \cdot r)\left(p^{\prime}\right)=p(f \cdot r)\left(p^{\prime}\right)=p f\left(r p^{\prime}\right)=\varphi(p \otimes f)\left(r p^{\prime}\right)=(\varphi(p \otimes f) \cdot r)\left(p^{\prime}\right),
$$

para todo $p^{\prime} \in P$. Logo, $\varphi$ é $R$-linear à direita e portanto $R$-bilinear. Seja $\left\{p_{i}, f_{i}\right\}_{i=1,2, \ldots, n}$ uma base dual de $P$. Dado $f \in \operatorname{End}\left(P_{R}\right)$, temos que $\sum_{i=1}^{n} f\left(p_{i} w\right) \otimes f_{i} \in P \otimes_{R}{ }^{*} P$ e

$$
\varphi\left(\sum_{i=1}^{n} f\left(p_{i}\right) \otimes f_{i}\right)\left(p^{\prime}\right)=\sum_{i=1}^{n} f\left(p_{i}\right) f_{i}\left(p^{\prime}\right)=\sum_{i=1}^{n} f\left(p_{i} f_{i}\left(p^{\prime}\right)\right)=f\left(p^{\prime}\right),
$$

para todo $p^{\prime} \in P$. Logo, $\varphi\left(\sum_{i=1}^{n} f\left(p_{i}\right) \otimes f_{i}\right)=f$ e portanto $\varphi$ é sobrejetora.

Vejamos que $\varphi$ é injetora. Sejam $p_{l}^{\prime} \in P$ e $f_{l}^{\prime} \in{ }^{*} P, \operatorname{com} l=1,2, \ldots, k$, tais que $\sum_{l=1}^{k} p_{l}^{\prime} f_{l}^{\prime}(p)=0$, para todo $p \in P$. Então,

$$
\sum_{l=1}^{k} p_{l}^{\prime} \otimes f_{l}^{\prime}=\sum_{l, i} p_{i} f_{i}\left(p_{l}^{\prime}\right) \otimes f_{l}^{\prime}=\sum_{l, i} p_{i} \otimes f_{i}\left(p_{l}^{\prime}\right) \cdot f_{l}^{\prime}=0
$$


pois

$$
\left(\sum_{l=1}^{k} f_{i}\left(p_{l}^{\prime}\right) \cdot f_{l}^{\prime}\right)(p)=\sum_{l=1}^{k} f_{i}\left(p_{l}^{\prime}\right) f_{l}^{\prime}(p)=\sum_{l=1}^{k} f_{i}\left(p_{l}^{\prime} f_{l}^{\prime}(p)\right)=0,
$$

para todo $p \in P$. Portanto, $\varphi$ é um isomorfismo de $R$-bimódulos.

(ii) Análogo ao item (i).

Lema 2.1.7. Sejam $P$ e $Q$ R-bimódulos. Se $P$ e $Q$ são $R$-módulos projetivos finitamente gerados à direita (à esquerda), então $P \otimes_{R} Q$ é um $R$-módulo projetivo finitamente gerado à direita (à esquerda).

Demonstração. Sejam $P$ e $Q R$-bimódulos que são projetivos e finitamente gerados como $R$-módulos à direita. Vamos considerar $\left\{f_{i}, p_{i}\right\}_{i=1,2, \ldots, n}$ uma base dual para $P$ e $\left\{g_{j}, q_{j}\right\}_{j=1,2, \ldots, m}$ uma base dual para Q. Defina

$$
\begin{aligned}
F_{i, j}: P \otimes_{R} Q & \longrightarrow R \\
p \otimes q & \longmapsto g_{j}\left(f_{i}(p) q\right) .
\end{aligned}
$$

Claramente $F_{i, j}$ está bem definida e é $R$-linear à direita, ou seja, $F_{i, j} \in{ }^{*}\left(P \otimes_{R} Q\right)$. Além disso, temos

$$
\begin{aligned}
\sum_{i, j=1}^{n, m}\left(p_{i} \otimes q_{j}\right) F_{i, j}(p \otimes q) & =\sum_{i, j=1}^{n, m}\left(p_{i} \otimes q_{j}\right) g_{j}\left(f_{i}(p) q\right)=\sum_{i, j=1}^{n, m} p_{i} \otimes q_{j} g_{j}\left(f_{i}(p) q\right) \\
& =\sum_{i=1}^{n} p_{i} \otimes f_{i}(p) q=\sum_{i=1}^{n} p_{i} f_{i}(p) \otimes q=p \otimes q,
\end{aligned}
$$

para todo $p \in P$ e $q \in Q$. Logo, $\left\{F_{i, j}, p_{i} \otimes q_{j}\right\}_{i, j}$ é uma base dual para $P \otimes_{R} Q$. Portanto, $P \otimes_{R} Q$ é um $R$-módulo à direita projetivo e finitamente gerado.

Lema 2.1.8. Sejam $P$ e $Q$ R-bimódulos.

(i) Se $P$ e $Q$ são projetivos e finitamente gerados como $R$-módulos à direita, então

$$
\begin{aligned}
\eta:{ }^{*} P \otimes_{R}{ }^{*} Q & \longrightarrow \\
f \otimes g & { }^{*}\left(Q \otimes_{R} P\right) \\
& (q \otimes p \mapsto f(g(q)) p)
\end{aligned}
$$

é um isomorfismo de R-bimódulos.

(ii) Se $P$ e $Q$ são projetivos e finitamente gerados como R-módulos à esquerda, então

$$
\begin{aligned}
\eta^{\prime}: P^{*} \otimes_{R} Q^{*} & \longrightarrow\left(Q \otimes_{R} P\right)^{*} \\
f \otimes g & \longmapsto(q \otimes p \mapsto g(q f(p)))
\end{aligned}
$$

é um isomorfismo de R-bimódulos.

Demonstração. (i) Vejamos primeiro que $\eta$ é um morfismo de $R$-bimódulos bem definido. Dado $r \in R$, temos

$$
\eta(f \otimes g)(q \otimes p r)=f(g(q) p r)=f(g(q) p) r=\eta(f \otimes g)(q \otimes p) r .
$$

Ou seja, $\eta(f \otimes g) \in^{*}\left(Q \otimes_{R} P\right)$. Temos também

$$
\eta(f \cdot r \otimes g)(q \otimes p)=(f \cdot r)(g(q) p)=f(r g(q) p)=\eta(f \otimes r \cdot g)(q \otimes p),
$$


para todo $r \in R, p \in P$ e $q \in Q$. Logo, $\eta$ é $R$-balanceada e portanto bem definida. Claramente, $\eta$ é $R$-linear à esquerda. Por outro lado,

$$
\eta(f \otimes g \cdot r)(q \otimes p)=f((g \cdot r)(q) p)=f(g(r q) p)=(\eta(f \otimes g) \cdot r)(q \otimes p),
$$

para $r \in R$. Assim, $\eta$ é $R$-linear à direita. Portanto, $\eta$ é um morfismo de $R$-bimódulos bem definido.

Sejam $\left\{p_{i}, f_{i}\right\}_{i=1,2, \ldots, n}$ base dual para $P$ e $\left\{q_{j}, g_{j}\right\}_{j=1,2, \ldots, m}$ base dual de $Q$. Vamos mostrar que

$$
\begin{array}{cl}
{ }^{*}\left(Q \otimes_{R} P\right) & \longrightarrow{ }^{*} P \otimes_{R}{ }^{*} Q \\
F & \longmapsto \\
\sum_{i, j} F\left(q_{j} \otimes p_{i}\right) \cdot f_{i} \otimes g_{j}
\end{array}
$$

é a inversa de $\eta$. De fato, dado $F \in{ }^{*}\left(Q \otimes_{R} P\right)$, temos

$$
\begin{aligned}
\eta\left(\sum_{i, j} F\left(q_{j} \otimes p_{i}\right) \cdot f_{i} \otimes g_{j}\right)(q \otimes p) & =\sum_{i, j} F\left(q_{j} \otimes p_{i}\right) f_{i}\left(g_{j}(q) p\right)=\sum_{i, j} F\left(q_{j} \otimes p_{i} f_{i}\left(g_{j}(q) p\right)\right) \\
& =\sum_{j} F\left(q_{j} \otimes g_{j}(q) p\right)=\sum_{j} F\left(q_{j} g_{j}(q) \otimes p\right)=F(q \otimes p),
\end{aligned}
$$

para todo $p \in P$ e $q \in Q$.

Por outro lado, dados $f \in{ }^{*} P$ e $g \in{ }^{*} Q$, temos

$$
\begin{aligned}
f \otimes g & \longmapsto(q \otimes p \mapsto f(g(q) p)) \longmapsto \sum_{i, j} f\left(g\left(q_{j}\right) p_{i}\right) f_{i} \otimes g_{j} \\
& =\sum_{i, j}\left(f \cdot g\left(q_{j}\right)\right)\left(p_{i}\right) f_{i} \otimes g_{j}=\sum_{j} f \cdot g\left(q_{j}\right) \otimes g_{j} \\
& =\sum_{j} f \otimes g\left(q_{j}\right) \cdot g_{j}=f \otimes g .
\end{aligned}
$$

Portanto, $\eta$ é isomorfismo de $R$-bimódulos.

(ii) Análogo ao item (i).

Um $R$-módulo $M$ à direita é dito plano se para qualquer sequência exata de $R$-módulos à esquerda

$$
0 \longrightarrow N^{\prime} \longrightarrow N \longrightarrow N^{\prime \prime} \longrightarrow 0
$$

temos que a sequência

$$
0 \longrightarrow M \otimes_{R} N^{\prime} \longrightarrow M \otimes_{R} N \longrightarrow M \otimes_{R} N^{\prime \prime} \longrightarrow 0
$$

é exata. Em particular, se $f: N^{\prime} \longrightarrow N$ é um isomorfismo de $R$-módulos à esquerda, então $M \otimes f$ : $M \otimes_{R} N^{\prime} \longrightarrow M \otimes_{R} N$ também é isomorfismo de grupos abelianos. Analogamente definimos $R$-módulo à esquerda plano.

Proposição 2.1.9. Todo módulo projetivo é plano.

Demonstração. [21, Proposição 19.16]. 
Os idempotentes centrais do anel $R$ tem um papel importante nesse trabalho. Vamos destacar aqui um isomorfismo trivial que será usando no próximos Capítulos. Sejam e um idempotente central em $R$ e $M$ um $R$-bimódulo. Denotamos $e M=\{m \in M ; e m=m\}$. Como $e$ é central em $R$, então $e M$ é um $R$-subbimódulo de $M$. Além disso, temos o isomorfismo de $R$-bimódulos

$$
\begin{aligned}
& R e \otimes_{R} M \longrightarrow e M \\
& r \otimes m \longmapsto r m
\end{aligned}
$$

cujo o inverso é dado por

$$
\begin{aligned}
e M & \longrightarrow R e \otimes_{R} M \\
m & \longmapsto e \otimes m .
\end{aligned}
$$

Analogamente, $M e=\{m \in M ; m e=m\}$ é um $R$-subbimódulo de $M$ e temos o isomorfismo de $R$ bimódulos

$$
\begin{aligned}
M \otimes_{R} R e & \longrightarrow M e \\
m \otimes r e & \longmapsto
\end{aligned}
$$

Sejam $M$ e $N R$-bimódulos, $F: M \longrightarrow N$ um isomorfismo de $R$-bimódulo e $e$ um idempotente central em $R$. A restrição de $F$ ao $R$-subbimódulo $e M$ também é um isomorfismo de $R$-bimódulos entre $e M$ e $e N$. Por abuso de notação, vamos denotar essa restrição também por $F$.

Vamos agora definir o grupo de Picard para um anel não comutativo. Essa definição é feita através de $R$-bimódulos inversíveis e pode ser encontrada em [23].

Definição 2.1.10. Um R-bimódulo $P$ é dito ser inversível se existem um $R$-bimódulo $Q$ e isomorfismos R-bilineares

$$
Q \otimes_{R} P \stackrel{\mathfrak{r}}{\longrightarrow} R \stackrel{\mathfrak{l}}{\longleftarrow} P \otimes_{R} Q
$$

O R-bimódulo $Q$ é chamado de inverso de $P$.

A próxima proposição, que está feita em um contexto um pouco mais geral, mostra que podemos escolher os isomorfismos $\mathfrak{r}$ e $\mathfrak{l}$ acima de tal forma que $\mathfrak{l} \otimes P=P \otimes \mathfrak{r}$ e $\mathfrak{r} \otimes Q=Q \otimes \mathfrak{l}$, ou seja, de tal forma que $(R, R, P, Q, \mathfrak{l}, \mathfrak{r})$ forme um contexto de Morita.

Proposição 2.1.11. Sejam $R$ e $S$ anéis, $P$ um $R$-S-bimódulo e $Q$ um $S$-R-bimódulo com

$$
\mathfrak{l}: P \otimes_{S} Q \longrightarrow R \text { e } \mathfrak{r}: Q \otimes_{R} P \longrightarrow S
$$

isomorfismos de R-bimódulos e S-bimódulos, respectivamente. Então, os funtores

$$
\begin{aligned}
& -\otimes_{R} P: \bmod -R \longrightarrow \bmod -S \\
& -\otimes_{S} Q: \bmod -S \longrightarrow \bmod -R
\end{aligned}
$$

formam uma equivalência de categorias. Além disso, podemos escolher os isomorfismos $\mathfrak{l}$ e $\mathfrak{r}$ de tal forma que os diagramas abaixo sejam comutativos
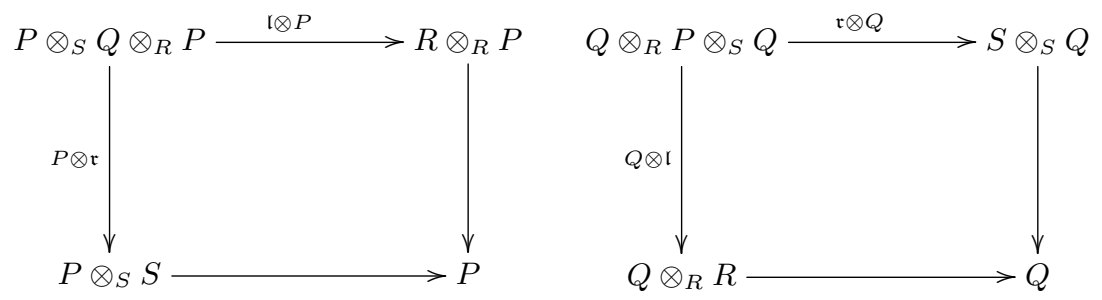

Ou seja, tal que

$$
\mathfrak{l}(p \otimes q) p^{\prime}=p \mathfrak{r}\left(q \otimes p^{\prime}\right) \quad e \quad \mathfrak{r}(q \otimes p) q^{\prime}=q \mathfrak{l}\left(p \otimes q^{\prime}\right),
$$

para todos $p, p^{\prime} \in P$ e $q, q^{\prime} \in Q$. 
Demonstração. Sejam $M, M^{\prime} \in \bmod -R$ e $\phi \in \operatorname{Hom}_{R}\left(M, M^{\prime}\right)$. É fácil ver que o diagrama

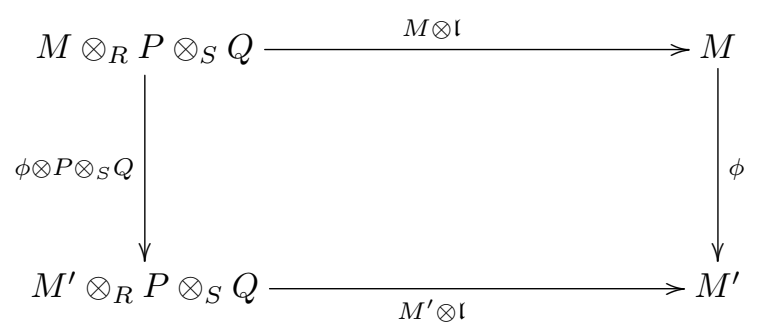

é comutativo. Logo, $-\otimes_{R} P \otimes_{S} Q \simeq I d_{\text {mod-R }}$. Analogamente, mostra-se que $-\otimes_{S} Q \otimes_{R} P \simeq I d_{\text {mod-S }}$. Portanto, temos uma equivalência de categorias.

Em particular, como $P \otimes_{S} Q \in \bmod -R$ e $Q \otimes_{R} P \in \bmod -S$ temos os diagramas comutativos
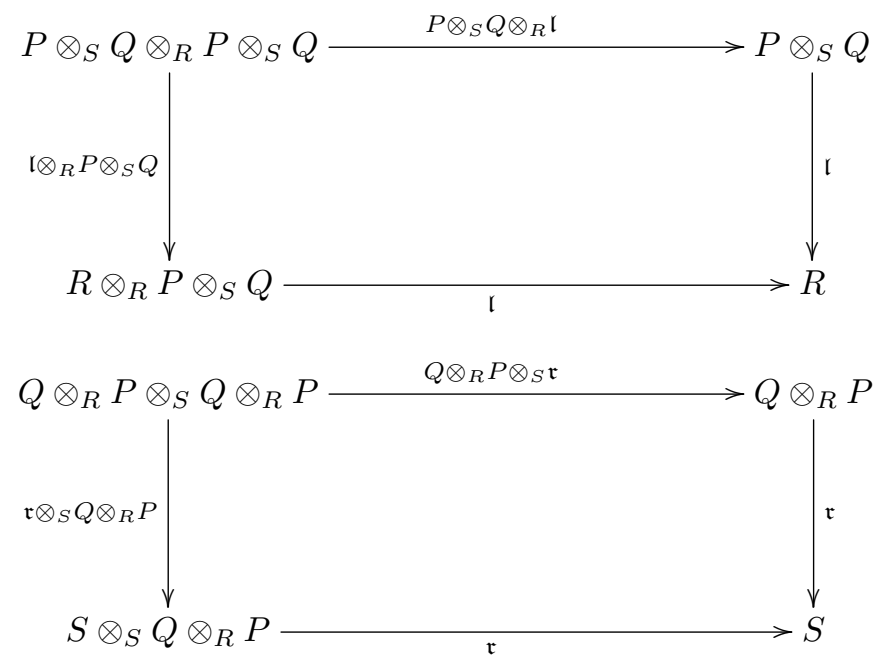

Então,

$$
P \otimes_{S} Q \otimes \mathfrak{l}=\mathfrak{l} \otimes_{R} P \otimes_{S} Q \text { e } Q \otimes_{R} P \otimes_{S} \mathfrak{r}=\mathfrak{r} \otimes_{S} Q \otimes_{R} P
$$

Segue que:

$$
\begin{aligned}
& \left(P \otimes_{S} \mathfrak{r}\right) \circ\left(P \otimes_{S} Q \otimes_{R} \mathfrak{l} \otimes_{R} P\right)=\left(\mathfrak{l} \otimes_{R} P\right) \circ\left(P \otimes_{S} \mathfrak{r} \otimes_{S} Q \otimes_{R} P\right), \\
& \left(\mathfrak{r} \otimes_{S} Q\right) \circ\left(Q \otimes_{R} \mathfrak{l} \otimes_{R} P \otimes_{S} Q\right)=\left(Q \otimes_{R} \mathfrak{l}\right) \circ\left(\mathfrak{r} \otimes_{S} Q \otimes_{R} P \otimes_{S} Q\right) .
\end{aligned}
$$

De fato, vamos verificar que vale (2.8). Sejam $p, p^{\prime}, p^{\prime \prime} \in P$ e $q, q^{\prime} \in Q$. Então,

$$
\begin{array}{ll} 
& \left(P \otimes_{S} \mathfrak{r}\right) \circ\left(P \otimes_{S} Q \otimes_{R} \mathfrak{l} \otimes_{R} P\right)\left(p \otimes q \otimes p^{\prime} \otimes q^{\prime} \otimes p^{\prime \prime}\right) \\
\stackrel{(2.7)}{=} & \left(P \otimes_{S} \mathfrak{r}\right) \circ\left(\mathfrak{l} \otimes_{R} P \otimes_{S} Q \otimes_{R} P\right)\left(p \otimes q \otimes p^{\prime} \otimes q^{\prime} \otimes p^{\prime \prime}\right) \\
= & \left(P \otimes_{S} \mathfrak{r}\right)\left(\mathfrak{l}(p \otimes q) p^{\prime} \otimes q^{\prime} \otimes p^{\prime \prime}\right) \\
= & \mathfrak{l}(p \otimes q) p^{\prime} \mathfrak{r}\left(q^{\prime} \otimes p^{\prime \prime}\right) \\
= & \left(\mathfrak{l} \otimes_{R} P\right)\left(p \otimes q \otimes p^{\prime} \mathfrak{r}\left(q^{\prime} \otimes p^{\prime \prime}\right)\right) \\
= & \left(\mathfrak{l} \otimes_{R} P\right) \circ\left(P \otimes_{S} Q \otimes_{R} P \otimes_{S} \mathfrak{r}\right)\left(p \otimes q \otimes p^{\prime} \otimes q^{\prime} \otimes p^{\prime \prime}\right) .
\end{array}
$$

Analogamente, mostra-se que vale (2.9).

Considere

$$
\widetilde{\mathfrak{l}}=\mathfrak{l} \circ\left(P \otimes_{S} \mathfrak{r} \otimes_{S} Q\right) \circ\left(P \otimes_{S} Q \otimes_{R} \mathfrak{l}^{-1}\right)
$$


Vejamos que substituindo $\mathfrak{l}$ por $\widetilde{\mathfrak{l}}$ os diagramas em (2.5) são comutativos:

$$
\begin{aligned}
\widetilde{\mathfrak{l}} \otimes_{R} P & =\left(\mathfrak{l} \circ\left(P \otimes_{S} \mathfrak{r} \otimes_{S} Q\right) \circ\left(P \otimes_{S} Q \otimes_{R} \mathfrak{l}^{-1}\right)\right) \otimes_{R} P \\
& =\left(\mathfrak{l} \otimes_{R} P\right) \circ\left(P \otimes_{S} \mathfrak{r} \otimes_{S} Q \otimes_{R} P\right) \circ\left(P \otimes_{S} Q \otimes_{R} \mathfrak{l}^{-1} \otimes_{R} P\right) \\
& \stackrel{(2.8)}{=}\left(P \otimes_{S} \mathfrak{r}\right) \circ\left(P \otimes_{S} Q \otimes_{R} \mathfrak{l} \otimes_{R} P\right) \circ\left(P \otimes_{S} Q \otimes_{R} \mathfrak{l}^{-1} \otimes_{R} P\right) \\
& =P \otimes_{S} \mathfrak{r} .
\end{aligned}
$$

Analogamente,

$$
\begin{aligned}
& Q \otimes_{R} \tilde{l}=Q \otimes_{R}\left(\mathfrak{l} \circ\left(P \otimes_{S} \mathfrak{r} \otimes_{S} Q\right) \circ\left(P \otimes_{S} Q \otimes_{R} \mathfrak{l}^{-1}\right)\right) \\
& =\left(Q \otimes_{R} \mathfrak{l}\right) \circ\left(Q \otimes_{R} P \otimes_{S} \mathfrak{r} \otimes_{S} Q\right) \circ\left(Q \otimes_{R} P \otimes_{S} Q \otimes_{R} \mathfrak{l}^{-1}\right) \\
& \stackrel{(2.7)}{=}\left(Q \otimes_{R} \mathfrak{l}\right) \circ\left(\mathfrak{r} \otimes_{S} Q \otimes_{R} P \otimes_{S} Q\right) \circ\left(Q \otimes_{R} P \otimes_{S} Q \otimes_{R} \mathfrak{l}^{-1}\right) \\
& \stackrel{(2.9)}{=}\left(\mathfrak{r} \otimes_{S} Q\right) \circ\left(Q \otimes_{R} \mathfrak{l} \otimes_{R} P \otimes_{S} Q\right) \circ\left(Q \otimes_{R} P \otimes_{S} Q \otimes_{R} \mathfrak{l}^{-1}\right) \\
& \stackrel{(2.7)}{=}\left(\mathfrak{r} \otimes_{S} Q\right) \circ\left(Q \otimes_{R} P \otimes_{S} Q \otimes_{R} \mathfrak{l}\right) \circ\left(Q \otimes_{R} P \otimes_{S} Q \otimes_{R} \mathfrak{l}^{-1}\right) \\
& =\mathfrak{r} \otimes_{S} Q .
\end{aligned}
$$

Portanto, os diagramas (2.5) são comutativos.

Analogamente, considerando $\widetilde{\mathfrak{r}}=\mathfrak{r} \circ\left(Q \otimes_{R} \mathfrak{l} \otimes_{R} P\right) \circ\left(Q \otimes_{R} P \otimes_{S} \mathfrak{r}^{-1}\right)$ temos que

$$
\mathfrak{l} \otimes_{R} P=P \otimes_{S} \widetilde{\mathfrak{r}} \quad \text { e } \quad Q \otimes_{R} \mathfrak{l}=\widetilde{\mathfrak{r}} \otimes_{S} Q
$$

Denotamos por $\operatorname{Pic}(R)$ o conjunto das classes de isomorfismo dos $R$-bimódulos inversíveis. Então, $\operatorname{Pic}(R)$ é um grupo, com a operação induzida pelo produto tensorial sobre $R$ e elemento neutro $[R]$, chamado grupo de Picard do anel $R$. Se $R$ é uma $K$-álgebra, onde $K$ é um anel comutativo, então denotamos por $\mathbf{P i c}_{K}(R)$ o subgrupo formado pelos elementos de $\mathbf{P i c}(R)$ que são centrais como $K$ módulos, ou seja,

$$
\operatorname{Pic}_{K}(R)=\{[P] \in \operatorname{Pic}(R) ; k p=p k \text { para todo } k \in K \text { e } p \in P\} .
$$

O próximo resultado, que é citado em [23], caracteriza os elementos do grupo de Picard.

Proposição 2.1.12. Seja $P$ um R-bimódulo. São equivalentes:

(i) P é inversivel.

(ii) $P$ é um $R$-módulo à direita projetivo, finitamente gerado e gerador e $R \simeq \operatorname{End}\left(P_{R}\right)$ é um isomorfismo de anéis via a ação à esquerda de $R$ sobre $P$.

(iii) $P$ é um $R$-módulo à esquerda projetivo, finitamente gerado e gerador e $R \simeq \operatorname{End}\left({ }_{R} P\right)$ é um isomorfismo de anéis via a ação à direita de $R$ sobre $P$.

Demonstração. $(i) \Rightarrow($ ii $)$ Seja $Q$ o inverso de $P$ e

$$
Q \otimes_{R} P \stackrel{\mathfrak{r}}{\longrightarrow} R \stackrel{\mathfrak{r}}{\longleftarrow} P \otimes_{R} Q
$$


Como $\mathfrak{l}$ é um isomorfismo, então existem $p_{i} \in P$ e $q_{i} \in Q$, com $i=1,2, \ldots, n$, tais que

$$
\mathfrak{l}\left(\sum_{i=1}^{n} p_{i} \otimes q_{i}\right)=1
$$

Defina

$$
\begin{aligned}
f_{i}: P & \longrightarrow R \\
p & \longmapsto \mathfrak{r}\left(q_{i} \otimes p\right)
\end{aligned} \in \operatorname{Hom}\left(P_{R}, R_{R}\right)
$$

para $i=1,2, \ldots, n$. Então, para todo $p \in P$ temos

$$
\sum_{i=1}^{n} p_{i} f_{i}(p)=\sum_{i=1}^{n} p_{i} \mathfrak{r}\left(q_{i} \otimes p\right) \stackrel{(2.6)}{=} \sum_{i=1}^{n} \mathfrak{r}\left(p_{i} \otimes q_{i}\right) p \stackrel{(2.10)}{=} 1 p=p .
$$

Logo, $P_{R}$ é um $R$-módulo projetivo e finitamente gerado.

Por $\mathfrak{r}$ ser isomorfismo existem $q_{j}^{\prime} \in Q$ e $p_{j}^{\prime} \in P$, com $j=1,2, \ldots, m$, tais que

$$
\mathfrak{r}\left(\sum_{j=1}^{m} q_{j}^{\prime} \otimes p_{j}^{\prime}\right)=1
$$

Defina $f_{j}^{\prime} \in \operatorname{Hom}\left(P_{R}, R_{R}\right)$ por

$$
\begin{aligned}
f_{j}^{\prime}: \quad P & \longrightarrow R \\
p & \longmapsto \mathfrak{r}\left(q_{j}^{\prime} \otimes p\right)
\end{aligned} \in \operatorname{Hom}\left(P_{R}, R_{R}\right) .
$$

Então,

$$
\sum_{j=1}^{m} f_{j}^{\prime}\left(p_{j}^{\prime}\right)=\sum_{j=1}^{m} \mathfrak{r}\left(q_{j}^{\prime} \otimes p_{j}^{\prime}\right) \stackrel{(2.11)}{=} 1
$$

Portanto, $P_{R}$ é um gerador.

Considere agora

$$
\begin{aligned}
\varphi: \quad & \longrightarrow \operatorname{End}\left(P_{R}\right) \\
r & \longmapsto(p \mapsto r p) .
\end{aligned}
$$

Claramente $\varphi$ está bem definida. Seja $r \in \operatorname{ker}(\varphi)$, então $r p=0$, para todo $p \in P$. Temos

$$
r=r 1 \stackrel{(2.10)}{=} r \mathfrak{l}\left(\sum_{i=1}^{n} p_{i} \otimes q_{i}\right)=\mathfrak{l}\left(\sum_{i=1}^{n} r p_{i} \otimes q_{i}\right)=0 .
$$

Logo, $\varphi$ é injetora. Considere agora $f \in \operatorname{End}\left(P_{R}\right)$, então $r=\sum_{i=1}^{n} \mathfrak{l}\left(f\left(p_{i}\right) \otimes q_{i}\right) \in R$ e temos

$$
\begin{aligned}
\varphi(r)(p) & =\sum_{i=1}^{n} \mathfrak{l}\left(f\left(p_{i}\right) \otimes q_{i}\right) p \stackrel{(2.6)}{=} \sum_{i=1}^{n} f\left(p_{i}\right) \mathfrak{r}\left(q_{i} \otimes p\right)=\sum_{i=1}^{n} f\left(p_{i} \mathfrak{r}\left(q_{i} \otimes p\right)\right) \\
& \stackrel{(2.6)}{=} \sum_{i=1}^{n} f\left(\mathfrak{l}\left(p_{i} \otimes q_{i}\right) p\right) \stackrel{(2.10)}{=} f(1 p)=f(p),
\end{aligned}
$$

para todo $p \in P$. Logo, $\varphi(r)=f$ e portanto $\varphi$ é um isomorfismo.

$($ ii $) \Rightarrow(i)$ Considere o $R$-bimódulo ${ }^{*} P=\operatorname{Hom}\left(P_{R}, R_{R}\right)$. Como $P_{R}$ é projetivo e finitamente gerado, segue do Lema 2.1.6 que $P \otimes_{R}{ }^{*} P \simeq R$ como $R$-bimódulos. Basta então construir um isomorfismo de ${ }^{*} P \otimes_{R} P \simeq R$. 
Por $P_{R}$ ser um gerador, existem $f_{i} \in{ }^{*} P$ e $p_{i} \in P$, com $i=1,2, \ldots, n$ tais que

$$
\sum_{i=1}^{n} f_{i}\left(p_{i}\right)=1
$$

A aplicação

$$
\begin{aligned}
\mathfrak{r}:{ }^{*} P \otimes_{R} P & \longrightarrow R \\
f \otimes p & \longmapsto f(p) .
\end{aligned}
$$

está bem definida e é $R$-bilinear. De fato, dado $r \in R$ temos que

$$
\mathfrak{r}(f \cdot r \otimes p)=(f \cdot r)(p)=f(r p)=\mathfrak{r}(f \otimes r p),
$$

para todo $r \in R, p \in P$ e $f \in{ }^{*} P$. Logo, $\mathfrak{r}$ está bem definida. Agora, para todo $r \in R, p \in P$ e $f \in{ }^{*} P$ temos

$$
\begin{gathered}
\mathfrak{r}(r \cdot f \otimes p)=(r \cdot f)(p)=r f(p)=r \mathfrak{r}(f \otimes p) . \\
\mathfrak{r}(f \otimes p r)=f(p r)=f(p) r=\mathfrak{r}(f \otimes p) r .
\end{gathered}
$$

Portanto, $\mathfrak{r}$ é $R$-bilinear.

Dado $r \in R$, temos

$$
\mathfrak{r}\left(\sum_{i=1}^{n} f_{i} \otimes p_{i} r\right)=\sum_{i=1}^{n} f_{i}\left(p_{i} r\right)=\sum_{i=1}^{n} f_{i}\left(p_{i}\right) \stackrel{(2.12)}{=} 1 r=r
$$

Logo, $\mathfrak{r}$ é sobrejetora. Vejamos que $\mathfrak{r}$ é injetora. Sejam $p_{l}^{\prime} \in P$ e $f_{l}^{\prime} \in{ }^{*} P$, com $l=1,2, \ldots, k$, tais que $\sum_{l=1}^{k} f_{l}^{\prime}\left(p_{l}^{\prime}\right)=0$. Defina $\theta_{l, i}: P_{R} \longrightarrow P_{R} \in \operatorname{End}\left(P_{R}\right)$ por $\theta_{l, i}(p)=p_{l}^{\prime} f_{i}(p)$, para todo $p \in P$, com $i=1,2, \ldots, n$ e $l=1,2, \ldots, k$. Como $\operatorname{End}\left(P_{R}\right) \simeq R$, via a ação à esquerda de $R$ sobre $P$, então existe $a_{l, i} \in R$ tal que $\theta_{l, i}(p)=a_{l, i} p$, para todo $p \in P$. Então, temos

$$
\begin{aligned}
\sum_{l=1}^{k} f_{l}^{\prime} \otimes p_{l}^{\prime} & =\sum_{l=1} f_{l}^{\prime} \otimes p_{l}^{\prime} 1=\sum_{l, i} f_{l}^{\prime} \otimes p_{l}^{\prime} f_{i}\left(p_{i}\right)=\sum_{l, i} f_{l}^{\prime} \otimes \theta_{l, i}\left(p_{i}\right) \\
& =\sum_{l, i} f_{l}^{\prime} \otimes a_{l, i} p_{i}=\sum_{l, i} f_{l}^{\prime} \cdot a_{l, i} \otimes p_{i}=0,
\end{aligned}
$$

pois, para todo $p \in P$, temos

$$
\begin{aligned}
\left(\sum_{l=1}^{k} f_{l}^{\prime} \cdot a_{l, i}\right)(p) & =\sum_{l=1}^{k} f_{l}^{\prime}\left(a_{l, i} p\right)=\sum_{l=1}^{k} f_{l}^{\prime}\left(\theta_{l, i}(p)\right) \\
& =\sum_{l=1}^{k} f_{l}^{\prime}\left(p_{l}^{\prime} f_{i}(p)\right)=\sum_{l=1}^{k} f_{l}^{\prime}\left(p_{l}^{\prime}\right) f_{i}(p)=0 .
\end{aligned}
$$

Portanto, $\mathfrak{r}$ é um isomorfismo de $R$-bimódulos.

$($ i $) \Leftrightarrow($ iii $)$ é completamente análogo.

Para referência futura, vamos destacar os isomorfismos construídos na Proposição 2.1.12:

Observação 2.1.13. Segue da prova da Proposição 2.1.12 que se $[P] \in \mathbf{P i c}(R)$, então existem isomorfismos de R-bimódulos 


$$
\begin{aligned}
& { }^{*} P \otimes_{R} P \stackrel{\mathfrak{r}}{\longrightarrow} R \quad R \quad P \otimes_{R}{ }^{*} P \stackrel{\mathfrak{l}}{\longrightarrow} \operatorname{End}\left(P_{R}\right) \simeq R \\
& f \otimes p \quad \longmapsto f(p)^{\prime}, \quad p \otimes f \quad \longmapsto \quad\left(p^{\prime} \mapsto p f\left(p^{\prime}\right)\right) \\
& P \otimes_{R} P^{*} \stackrel{\mathfrak{l}^{\prime}}{\longrightarrow} R \quad R \quad P^{*} \otimes_{R} P \stackrel{\stackrel{\mathfrak{r}^{\prime}}{\longrightarrow}}{\longrightarrow} \operatorname{End}\left({ }_{R} P\right) \simeq R \\
& p \otimes f \quad \longmapsto f(p) \quad e \quad f \otimes p \quad \longmapsto \quad\left(p^{\prime} \mapsto f\left(p^{\prime}\right) p\right)
\end{aligned}
$$

Observação 2.1.14. Se $[P] \in \mathbf{P i c}(R)$, por [24, Teorema 1.1], ${ }^{*} P \simeq P^{*}$ como R-bimódulos. Pela demonstração da Proposição 2.1.12, temos que $\left[{ }^{*} P\right]=\left[P^{*}\right]=[P]^{-1}$ em $\mathbf{P i c}(R)$. Em geral, vamos denotar $[P]^{-1}=\left[P^{-1}\right]$ e vamos considerar os isomorfismos $P \otimes_{R} P^{-1} \stackrel{\mathfrak{l}}{\longrightarrow} R \stackrel{\mathfrak{r}}{\longleftarrow} P^{-1} \otimes_{R} P$ satisfazendo as igualdades em (2.6).

Observação 2.1.15. Se R é comutativo $e[P] \in \mathbf{P i c}_{R}(R)$, então $P$ é um $R$-módulo projetivo, finitamente gerado e fiel. Por [19, Lema I.5.1] a existência do isomorfismo $R \simeq \operatorname{End}(P)$ é equivalente à rank $k_{R}(P)=1$. Portanto,

$\operatorname{Pic}_{R}(R)=\{[P] ; P$ é R-módulo (central) projetivo e finitamente gerado com posto 1$\}$.

É bem conhecido (ver, por exemplo, [20, página 35]) que para uma extensão de anéis comutativos $R \subseteq S$, se $[P] \in \mathbf{P i c}(R)$, então $\left[P \otimes_{R} S\right] \in \mathbf{P i c}(S)$. Nos próximos resultados vamos mostrar uma condição para que o mesmo também seja válido para extensão de anéis não comutativos. O próximo Lema foi apresentado em [25] (ver Equações 5.1 e 5.2) e dá uma condição para construir estrutura de $S$-bimódulo a partir de um $R$-bimódulo $P$ e um $S$-bimódulo $M$.

Lema 2.1.16. Sejam $R \subseteq S$ uma extensão de anéis com mesma unidade e $P$ um $R$-bimódulo. Denote por $\mu$ a multiplicação em $S$.

(i) Seja $\rho: S \otimes_{R} P \longrightarrow P \otimes_{R} S$ uma aplicação R-bilinear tal que

$$
\begin{gathered}
(P \otimes \mu) \circ(\rho \otimes S) \circ(S \otimes \rho)=\rho \circ(\mu \otimes P) \\
\rho(1 \otimes p)=p \otimes 1, \quad \text { para todo } p \in P,
\end{gathered}
$$

Se $M$ é um $S$-módulo unital à esquerda, então $P \otimes_{R} M$ tem uma estrutura de $S$-módulo à esquerda dada por

$$
s *(p \otimes m)=\sum_{i=1}^{n} p_{i} \otimes s_{i} m,
$$

onde $\rho(s \otimes p)=\sum_{i=1}^{n} p_{i} \otimes s_{i}$. Além disso, se $M$ é um $S$-bimódulo unital, então $P \otimes_{R} M$ é um $S$-bimódulo unital, onde a estrutura de S-módulo à direita è dada pela estrutura de $M$.

(ii) Seja $\rho^{\prime}: P \otimes_{R} S \longrightarrow S \otimes_{R} P$ uma aplicação R-bilinear tal que

$$
\begin{gathered}
(\mu \otimes P) \circ\left(S \otimes \rho 1^{\prime}\right) \circ\left(\rho^{\prime} \otimes S\right)=\rho^{\prime} \circ(P \otimes \mu) \\
\rho(p \otimes 1)=1 \otimes p, \quad \text { para todo } p \in P,
\end{gathered}
$$

Se $M$ é um $S$-módulo unital à direita, então $M \otimes_{R} R P$ tem uma estrutura de $S$-módulo à direita dada por

$$
(m \otimes p) * s=\sum_{i=1}^{n} m s_{i} \otimes p_{i},
$$

onde $\rho^{\prime}(p \otimes s)=\sum_{i=1}^{n} s_{i} \otimes p_{i}$. Além disso, se $M$ é um $S$-bimódulo unital, então $M \otimes_{R} P$ é um S-bimódulo unital, onde a estrutura de S-módulo à direita è dada pela estrutura de $M$. 
Demonstração. (i) Dados $p \in P$ e $s, s^{\prime} \in S$ denote

$$
\rho(s \otimes p)=\sum_{i=1}^{n} p_{i} \otimes s_{i} \text { e } \rho\left(s^{\prime} \otimes p_{i}\right)=\sum_{j=1}^{m} p_{i, j} \otimes s_{j}^{\prime} .
$$

Então,

$$
s^{\prime} *(s *(p \otimes m))=s^{\prime} *\left(\sum_{i=1}^{n} p_{i} \otimes s_{i} m\right)=\sum_{i, j} p_{i, j} \otimes s_{j}^{\prime}\left(s_{i} m\right) .
$$

Por (2.13) temos

$$
\begin{aligned}
\rho\left(s^{\prime} s \otimes p\right) & =\rho \circ(\mu \otimes P)\left(s^{\prime} \otimes s \otimes p\right) \\
& =(P \otimes \mu) \circ(\rho \otimes S) \circ(S \otimes \rho)\left(s^{\prime} \otimes s \otimes p\right) \\
& =\sum_{i=1}^{n}(P \otimes \mu) \circ(\rho \otimes S)\left(s^{\prime} \otimes p_{i} \otimes s_{i}\right) \\
& =\sum_{i, j}(P \otimes \mu)\left(p_{i, j} \otimes s_{j}^{\prime} \otimes s_{i}\right) \\
& =\sum_{i, j} p_{i, j} \otimes s_{j}^{\prime} s_{i} .
\end{aligned}
$$

Então,

$$
\left(s^{\prime} s\right) *(p \otimes m)=\sum_{i, j} p_{i, j} \otimes\left(s_{j}^{\prime} s_{i}\right) m=\sum_{i, j} p_{i, j} \otimes s_{j}^{\prime}\left(s_{i} m\right)=s^{\prime} *(s *(p \otimes m)) .
$$

Além disso, por (2.14) temos

$$
1 *(p \otimes m)=p \otimes 1 m=p \otimes m .
$$

Logo, $P \otimes_{R} M$ é um $S$-módulo à esquerda.

Suponha que $M$ seja um $S$-bimódulo e considere em $P \otimes_{R} M$ a ação à direita induzida pela de $M$, ou seja, $(p \otimes m) * s=p \otimes m s$, para todo $m \in M, p \in P$ e $s \in S$. Assim, dados $s, s^{\prime} \in S, p \in P$ e $m \in M$, denote $\rho(s \otimes p)=\sum_{i=1}^{n} p_{i} \otimes s_{i}$, então

$$
\begin{aligned}
(s *(p \otimes m)) * s^{\prime} & =\left(\sum_{i=1}^{n} p_{i} \otimes s_{i} m\right) * s^{\prime}=\sum_{i=1}^{n} p_{i} \otimes\left(s_{i} m\right) s^{\prime} \\
& =\sum_{i=1}^{n} p_{i} \otimes s_{i}\left(m s^{\prime}\right)=s *\left(p \otimes m s^{\prime}\right) \\
& =s *\left((p \otimes m) * s^{\prime}\right) .
\end{aligned}
$$

Portanto, $P \otimes_{R} M$ é um $S$-bimódulo unital.

(ii) Análogo ao item $(i)$.

Exemplo 2.1.17. Seja $P$ é um $\mathcal{Z}$-bimódulo central. Como $R$ é um $\mathcal{Z}$-bimódulo central, então existe um isomorfismo de $\mathcal{Z}$-bimódulos $\rho: R \otimes_{\mathcal{Z}} P \longrightarrow P \otimes_{\mathcal{Z}} R$ definido por $\rho(r \otimes p)=p \otimes r$. Vejamos que $\rho$ satisfaz as condições do Lema 2.1.16. Sejam $r, r^{\prime} \in R$ e $p \in P$, então:

$$
\begin{aligned}
(P \otimes m) \circ(\rho \otimes R) \circ(R \otimes \rho)\left(r \otimes r^{\prime} \otimes p\right) & =(P \otimes m) \circ(\rho \otimes R)\left(r \otimes p \otimes r^{\prime}\right) \\
& =(P \otimes m)\left(p \otimes r \otimes r^{\prime}\right) \\
& =p \otimes r r^{\prime} \\
& =\rho\left(r r^{\prime} \otimes p\right) \\
& =\rho \circ(m \otimes P)\left(r \otimes r^{\prime} \otimes p\right) .
\end{aligned}
$$


Logo, $\rho$ satisfaz (2.13). A condições (2.14) segue diretamente da definição de $\rho$. Pelo Lema 2.1.16, para qualquer R-bimódulo $M$, o produto tensorial $P \otimes_{\mathcal{Z}} M$ tem estrutura de R-bimódulo definida por

$$
r_{1} *(p \otimes m) * r_{2}=p \otimes r_{1} m r_{2},
$$

para todo $p \in P, m \in M$ e $r_{1}, r_{2} \in R$.

Lema 2.1.18. Sejam $R \subseteq S$ uma extensão de anéis com mesma unidade, $P$ um R-bimódulo unital e $\rho: S \otimes_{R} P \longrightarrow P \otimes_{R} S$ um morfismo de R-bimódulos que satisfaz as condições (2.13) e (2.14). Seja $M$ um $S$-bimódulo, então existe um isomorfismo de $S$-bimódulos

$$
\begin{aligned}
& \eta: \quad\left(P \otimes_{R} S\right) \otimes_{S} M \longrightarrow P \otimes_{R} M \\
& \left(p \otimes_{R} s\right) \otimes_{S} m \longmapsto p \otimes_{R} s m,
\end{aligned}
$$

onde $P \otimes_{R} S$ e $P \otimes_{R} M$ são $S$-bimódulos com as ações dados no Lema 2.1.16.

Demonstração. Claramente $\eta$ está bem definida e é $S$-linear à direita. Dados $s, s^{\prime} \in S, p \in P$ e $m \in M$, denote $\rho\left(s^{\prime} \otimes_{R} p\right)=\sum_{i=1}^{n} p_{i} \otimes_{R} s_{i}^{\prime}$. então,

$$
\begin{aligned}
\eta\left(s^{\prime} *\left(\left(p \otimes_{R} s\right) \otimes_{S} m\right)\right) & =\eta\left(\sum_{i=1}^{n} p_{i} \otimes_{R} s_{i}^{\prime} s \otimes_{S} m\right)=\sum_{i=1}^{n} p_{i} \otimes_{R}\left(s_{i}^{\prime} s\right) m \\
& =\sum_{i=1}^{n} p_{i} \otimes_{R} s_{i}^{\prime}(s m)=s^{\prime} *\left(p \otimes_{R} s m\right) \\
& =s^{\prime} \eta\left(\left(p \otimes_{R} s\right) \otimes_{S} m\right) .
\end{aligned}
$$

Logo, $\eta$ é $S$-bilinear. Sua inversa é dada por:

$$
\begin{aligned}
\eta^{-1}: P \otimes_{R} M & \longrightarrow\left(P \otimes_{R} S\right) \otimes_{S} M \\
p \otimes_{R} m & \longmapsto p \otimes_{R} 1 \otimes_{S} m .
\end{aligned}
$$

De fato, sejam $p \in P, m \in M$ e $s \in S$, então

$$
\eta^{-1}\left(\eta\left(p \otimes_{R} s \otimes_{S} m\right)\right)=\eta^{-1}\left(p \otimes_{R} s m\right)=p \otimes_{R} 1 \otimes_{S} s m=p \otimes_{R} s \otimes_{S} m .
$$

Por outro lado,

$$
\eta\left(\eta^{-1}\left(p \otimes_{R} m\right)\right)=\eta\left(p \otimes_{R} 1 \otimes_{S} m\right)=p \otimes_{R} 1 m=p \otimes_{R} m .
$$

Portanto, $\eta$ é isomorfismo de $S$-bimódulos.

Lema 2.1.19. Sejam $R \subseteq S$ uma extensão de anéis com mesma unidade, $[P] \in \operatorname{Pic}(R)$ e $\rho: S \otimes_{R} P \longrightarrow$ $P \otimes_{R} S$ um isomorfismo de R-bimódulos tal que $\rho$ satisfaz as condições do Lema 2.1.16. Denote $\left[P^{-1}\right]$ o inverso de $[P]$ em $\mathbf{P i c}(R)$ e $P \otimes_{R} P^{-1} \stackrel{\mathfrak{l}}{\longrightarrow} R \stackrel{\mathfrak{r}}{\longleftarrow} P^{-1} \otimes_{R} P$ isomorfismos de R-bimódulos.

(i) Considere o isomorfismo de R-bimódulos $\bar{\rho}: S \otimes_{R} P^{-1} \longrightarrow P^{-1} \otimes_{R} S$ induzido por $\bar{\rho}=P^{-1} \otimes \rho^{-1} \otimes$ $P^{-1}$, ou seja,

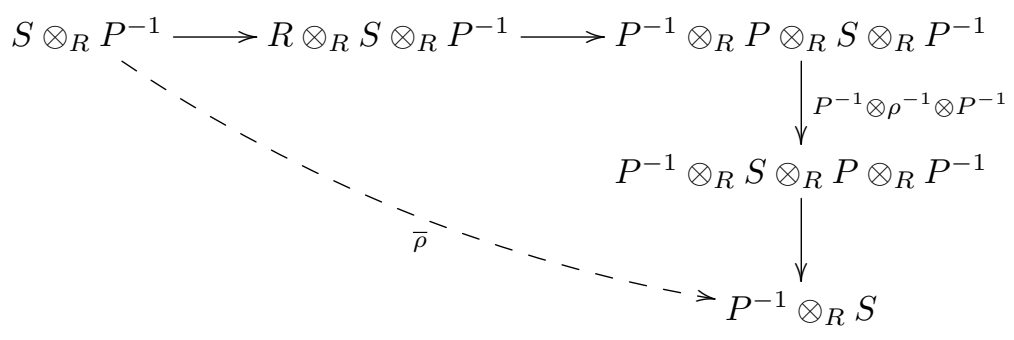


Suponha que $\bar{\rho}$ satisfaz as condições do Lema 2.1.16 e considere $P^{-1} \otimes_{R} S$ com a estrutura de $S$-bimódulo definida por $\bar{\rho}$ como no Lema 2.1.16. Seja $\Psi: P \otimes_{R}\left(P^{-1} \otimes_{R} S\right) \longrightarrow S$ o isomorfismo definido por

$$
\Psi: P \otimes_{R}\left(P^{-1} \otimes_{R} S\right) \stackrel{P \otimes \bar{\rho}^{-1}}{\longrightarrow} P \otimes_{R}\left(S \otimes_{R} P^{-1}\right) \stackrel{\rho^{-1} \otimes P^{-1}}{\longrightarrow} S \otimes_{R} P \otimes P^{-1} \stackrel{S \otimes \mathfrak{l}}{\longrightarrow} S,
$$

ou seja, $\Psi=(S \otimes \mathfrak{l}) \circ\left(\rho^{-1} \otimes P^{-1}\right) \circ\left(P \otimes \bar{\rho}^{-1}\right)$, então

$$
\Psi(p \otimes \bar{p} \otimes s)=\mathfrak{l}(p \otimes \bar{p}) s
$$

para todo $s \in S, p \in P$ e $\bar{p} \in P^{-1}$.

(ii) Sejam $s \in S, p \in P$ e $\bar{p} \in P^{-1}$. Considere $\sum_{k=1}^{l} \mathfrak{l}\left(p_{k} \otimes \bar{p}_{k}\right)=1$ e denote $\rho(s \otimes p)=\sum_{i=1}^{n} p_{i} \otimes s_{i} e$ $\rho^{-1}\left(p_{k} \otimes s_{i}\right)=\sum_{j=1}^{m} s_{i, j} \otimes \bar{p}_{k, j}$. Então,

$$
s \mathfrak{l}(p \otimes \bar{p})=\sum_{i, j, k} \mathfrak{l}\left(p_{i} \otimes \bar{p}_{k}\right) s_{i, j} \mathfrak{l}\left(p_{k, j} \otimes \bar{p}\right) .
$$

(iii) $\Psi$ é isomorfismo de S-bimódulos.

(iv) $\left[P \otimes_{R} S\right] \in \mathbf{P i c}(S)$

Demonstração. Dados $s \in S$ e $\bar{p} \in P^{-1}$. Considere $p_{l} \in P$ e $\bar{p}_{l} \in P^{-1}$, com $l=1,2, \ldots, n$, tais que $\sum_{l=1}^{n} \mathfrak{r}\left(\bar{p}_{l} \otimes p_{l}\right)=1$. Denotando $\rho^{-1}\left(p_{l} \otimes s\right)=\sum_{i=1}^{m} s_{i} \otimes p_{l, i}$, temos

$$
\bar{\rho}(s \otimes \bar{p})=\sum_{l, i} \bar{p}_{l} \otimes s_{i} \mathfrak{l}\left(p_{l, i} \otimes \bar{p}\right)
$$

Sua inversa é dada por

$$
\bar{\rho}^{-1}(\bar{p} \otimes s)=\sum_{k, j} \mathfrak{r}\left(\bar{p} \otimes p_{k, j}\right) s_{j} \otimes \bar{p}_{k}
$$

onde $\sum_{k=1}^{l} \mathfrak{l}\left(p_{k} \otimes \bar{p}_{k}\right)=1$ e $\rho\left(s \otimes p_{k}\right)=\sum_{j=1}^{m} p_{k, j} \otimes s_{j}$

(i) Sejam $s \in S, p \in P$ e $\bar{p} \in P^{-1}$. Considere $\sum_{k=1}^{n} \mathfrak{l}\left(p_{k} \otimes \bar{p}_{k}\right)=1$ e denote $\rho\left(s \otimes p_{k}\right)=\sum_{j=1}^{m} p_{k, j} \otimes s_{j}$. 
Então,

$$
\begin{aligned}
& p \otimes \bar{p} \otimes s \quad \stackrel{P \otimes \bar{\rho}^{-1}}{\longmapsto} \quad \sum_{k, j} p \otimes \mathfrak{r}\left(\bar{p} \otimes p_{k, j}\right) s_{j} \otimes \bar{p}_{k} \\
& \stackrel{\rho^{-1} \otimes P^{-1}}{\longmapsto} \sum_{k, j} \rho^{-1}\left(p \mathfrak{r}\left(\bar{p} \otimes p_{k, j}\right) \otimes s_{j}\right) \otimes \bar{p}_{k} \\
& =\quad \sum_{k, j} \rho^{-1}\left(\mathfrak{l}(p \otimes \bar{p}) p_{k, j} \otimes s_{j}\right) \otimes \bar{p}_{k} \\
& =\quad \sum_{k, j} \mathfrak{l}(p \otimes \bar{p}) \rho^{-1}\left(p_{k, j} \otimes s_{j}\right) \otimes \bar{p}_{k} \\
& =\quad \sum_{k} \mathfrak{r}(p \otimes \bar{p}) s \otimes p_{k} \otimes \bar{p}_{k} \\
& \stackrel{S \otimes \mathfrak{l}}{\longmapsto} \quad \sum_{k} \mathfrak{l}(p \otimes \bar{p}) s \mathfrak{l}\left(p_{k} \otimes \bar{p}_{k}\right) \\
& =\quad \mathfrak{l}(p \otimes \bar{p}) s .
\end{aligned}
$$

Logo,

$$
\Psi(p \otimes \bar{p} \otimes s)=(S \otimes \mathfrak{l}) \circ\left(\rho^{-1} \otimes P^{-1}\right) \circ\left(P \otimes \bar{\rho}^{-1}\right)(p \otimes \bar{p} \otimes s)=\mathfrak{l}(p \otimes \bar{p}) s .
$$

(ii) Vamos calcular $\Psi^{-1}(s \mathfrak{l}(p \otimes \bar{p}))$ : observe que $\Psi^{-1}=(P \otimes \bar{\rho}) \circ\left(\rho \otimes P^{-1}\right) \circ\left(S \otimes \mathfrak{l}^{-1}\right)$. Então, se $\sum_{k=1}^{n} \mathfrak{r}\left(p_{k} \otimes \bar{p}_{k}\right)=1$, temos

$$
\begin{aligned}
s \mathfrak{l}(p \otimes \bar{p}) & \stackrel{S \otimes \mathfrak{r}^{-1}}{\longmapsto} \sum_{k=1}^{l} s \mathfrak{l}(p \otimes \bar{p}) \otimes p_{k} \otimes \bar{p}_{k}=\sum_{k=1}^{l} s \otimes \mathfrak{l}(p \otimes \bar{p}) p_{k} \otimes \bar{p}_{k} \\
& =\sum_{k=1}^{l} s \otimes p \mathfrak{r}\left(\bar{p} \otimes p_{k}\right) \otimes \bar{p}_{k}=\sum_{k=1}^{l} s \otimes p \otimes \mathfrak{r}\left(\bar{p} \otimes p_{k}\right) \bar{p}_{k} \\
& =\sum_{k=1}^{l} s \otimes p \otimes \bar{p} \mathfrak{l}\left(p_{k} \otimes \bar{p}_{k}\right)=s \otimes p \otimes \bar{p} \\
& \stackrel{\rho \otimes P^{-1}}{\longmapsto} \sum_{i=1}^{n} p_{i} \otimes s_{i} \otimes \bar{p} \stackrel{P \otimes \bar{\rho}}{\longmapsto} \sum_{i, j, k} p_{i} \otimes \bar{p}_{k} \otimes s_{i, j} \mathfrak{l}\left(p_{k, j} \otimes \bar{p}\right) .
\end{aligned}
$$

Ou seja, $\Psi^{-1}(s \mathfrak{l}(p \otimes \bar{p}))=\sum_{i, j, k} p_{i} \otimes \bar{p}_{k} \otimes s_{i, j} \mathfrak{l}\left(p_{k, j} \otimes \bar{p}\right)$. Portanto,

$$
\begin{aligned}
s \mathfrak{l}(p \otimes \bar{p}) & =\Psi \Psi^{-1}(s \mathfrak{l}(p \otimes \bar{p}))=\Psi\left(\sum_{i, j, k} p_{i} \otimes \bar{p}_{k} \otimes s_{i, j} \mathfrak{l}\left(p_{k, j} \otimes \bar{p}\right)\right) \\
& =\sum_{i, j, k} \mathfrak{l}\left(p_{i} \otimes \bar{p}_{k}\right) s_{i, j} \mathfrak{l}\left(p_{k, j} \otimes \bar{p}\right) .
\end{aligned}
$$

(iii) Basta verificar que $\Psi$ é $S$-linear à esquerda. Sejam $s, s^{\prime} \in S, p \in P$ e $\bar{p} \in P^{-1}$. Sejam $p_{k} \in P$ e $\bar{p}_{k} \in P^{-1}$, tais que $\sum_{k=1}^{l} \mathfrak{l}\left(p_{k} \otimes \bar{p}_{k}\right)=1$ e denote $\rho\left(s^{\prime} \otimes p\right)=\sum_{i=1}^{n} p_{i} \otimes s_{i}^{\prime}$ e $\rho^{-1}\left(p_{k} \otimes s_{i}^{\prime}\right)=\sum_{j=1}^{l} s_{i, j}^{\prime} \otimes p_{k, j}$. 
Então, $\bar{\rho}\left(s_{i}^{\prime} \otimes \bar{p}\right)=\sum_{k, l} \bar{p}_{k} \otimes s_{i, j}^{\prime} \mathfrak{l}\left(p_{k, j} \otimes \bar{p}\right)$. Assim,

$$
\begin{aligned}
\Psi\left(s^{\prime} *(p \otimes(\bar{p} \otimes s))\right) & =\Psi\left(\sum_{i=1}^{n} p_{i} \otimes s_{i}^{\prime} *(\bar{p} \otimes s)\right) \\
& =\Psi\left(\sum_{i, j, k} p_{i} \otimes \bar{p}_{k} \otimes s_{i, j}^{\prime} \mathfrak{l}\left(p_{k, j} \otimes \bar{p}\right) s\right) \\
& =\sum_{i, j, k} \mathfrak{l}\left(p_{i} \otimes \bar{p}_{k}\right) s_{i, j}^{\prime} \mathfrak{l}\left(p_{k, j} \otimes \bar{p}\right) s \\
\stackrel{(2.17)}{=} & s^{\prime} \mathfrak{l}(p \otimes \bar{p}) s=s^{\prime} \Psi(p \otimes \bar{p} \otimes s) .
\end{aligned}
$$

(iv) Pelo Lema 2.1.18 e pelo item (iii) temos os isomorfismos de $S$-bimódulos

$$
\left(P \otimes_{R} S\right) \otimes_{S}\left(P^{-1} \otimes_{R} S\right) \simeq P \otimes_{R}\left(P^{-1} \otimes_{R} S\right) \simeq S
$$

Analogamente, $\left(P^{-1} \otimes_{R} S\right) \otimes_{S}\left(P \otimes_{R} S\right) \simeq S$ como $S$-bimódulos. Portanto, $\left[P \otimes_{R} S\right] \in \mathbf{P i c}(S)$.

\subsection{O semigrupo de Picard generalizado}

Seja $R$ um anel com unidade. Dizemos que um $R$-bimódulo $P$ é parcialmente inversível se

(i) $P$ é um $R$-módulo projetivo e finitamente gerado como módulo à direita e à esquerda;

(ii) As aplicações

$$
\begin{aligned}
& R \longrightarrow \operatorname{End}\left(P_{R}\right) \\
& r \longmapsto(p \mapsto r p)
\end{aligned} \quad \text { e } \quad \begin{aligned}
& R \\
& r
\end{aligned} \quad \begin{aligned}
& \operatorname{End}\left({ }_{R} P\right) \\
& (p \mapsto p r)
\end{aligned}
$$

são sobrejetoras.

Vamos denotar por $\mathbf{P i c S}(R)$ o conjunto das classes de isomorfismo de $R$-bimódulos parcialmente inversíveis, ou seja,

$\operatorname{PicS}(R)=\{[P], P$ é um $R$-bimódulo parcialmente inversível. $\}$.

Proposição 2.2.1. $\operatorname{PicS}(R)$ é um monoide com o produto $[P][Q]=\left[P \otimes_{R} Q\right]$.

Demonstração. Pelo Lema 2.1.7, temos que $P \otimes_{R} Q$ é projetivo e finitamente gerado com $R$-módulo à direita e à esquerda. 
Considere $\psi$ dado pela cadeia de morfismos de $R$-bimódulos

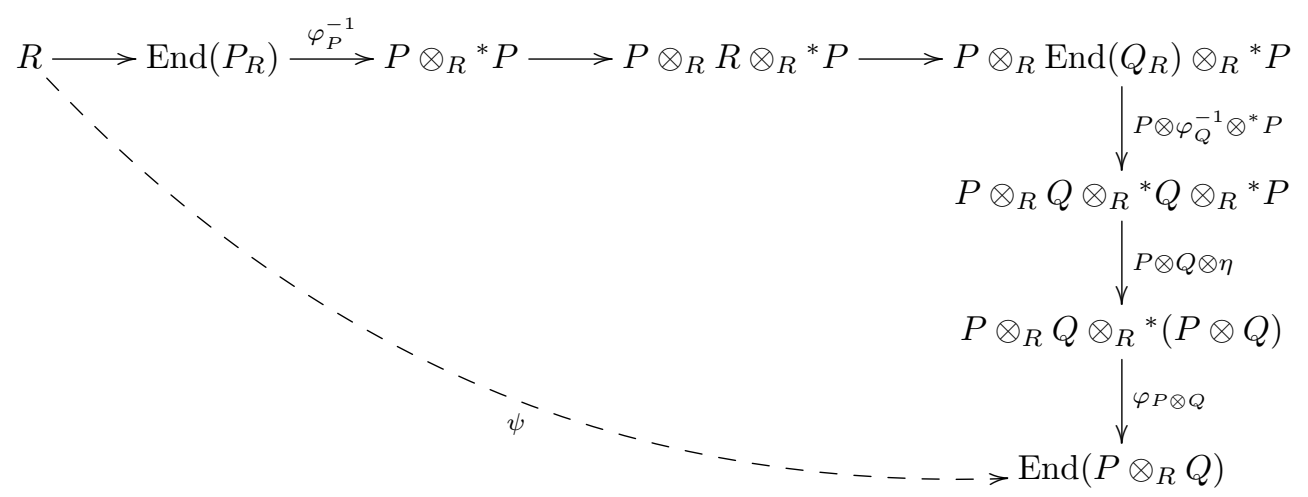

onde o $\varphi_{P}, \varphi_{Q}$ e $\varphi_{P \otimes Q}$ são isomorfismos como no Lema 2.1.6 e $\eta$ é isomorfismo dado no Lema 2.1.8. Então $\psi$ é sobrejetor e é dado por

$$
\psi(r)(p \otimes q)=r p \otimes q .
$$

De fato, sejam $\left\{p_{i}, f_{i}\right\}_{i=1, \ldots, n}$ base dual de $P$ como $R$-módulo à direita e $\left\{q_{j}, g_{j}\right\}_{j=1, \ldots, m}$ base dual de $Q$ como $R$-módulo á direita. Dado $r \in R$, temos:

$$
\begin{aligned}
r \longmapsto & (p \mapsto r p) \longmapsto \sum_{i=1}^{n} r p_{i} \otimes f_{i} \longmapsto \sum_{i=1}^{n} r p_{i} \otimes 1 \otimes f_{i} \\
\longmapsto & \sum_{i=1}^{n} r p_{i} \otimes I d_{Q} \otimes f_{i} \longmapsto \sum_{i, j} r p_{i} \otimes q_{j} \otimes g_{j} \otimes f_{i} \\
\longmapsto & \sum_{i, j} r p_{i} \otimes q_{j} \otimes\left((p \otimes q) \mapsto g_{j}\left(f_{i}(p) q\right)\right) \\
\longmapsto & {\left[p \otimes q \mapsto \sum_{i, j} r p_{i} \otimes q_{j} g_{j}\left(f_{i}(p) q\right)\right] } \\
= & {\left[p \otimes q \mapsto \sum_{i=1}^{n} r p_{i} \otimes f_{i}(p) q\right] } \\
= & {\left[p \otimes q \mapsto \sum_{i=1}^{n} r p_{i} f_{i}(p) \otimes q\right] } \\
= & {[p \otimes q \mapsto r p \otimes q] . }
\end{aligned}
$$

Analogamente, $\psi^{\prime}: R \longrightarrow \operatorname{End}\left({ }_{R} P \otimes Q\right)$ definida por

$$
\varphi^{\prime}(r)(p \otimes q)=p \otimes q r
$$

é sobrejetor. Logo, $\left[P \otimes_{R} Q\right] \in \mathbf{P i c S}(R)$. Claramente, $[R]$ é o elemento neutro de $\mathbf{P i c S}(R)$. Portanto, $\operatorname{PicS}(R)$ é um monoide.

Observação 2.2.2. $\mathcal{U}(\operatorname{PicS}(R))=\operatorname{Pic}(R)$.

Se $R$ é uma $K$-álgebra, onde $K$ é um anel comutativo, denotamos $\operatorname{PicS}_{K}(R)$ o subsemigrupo formado pelos $[P] \in \mathbf{P i c S}(R)$ que são centrais como $K$-bimódulos. 
Se $R$ é comutativo, então $\mathbf{P i c S}(R)=\mathbf{P i c S}_{R}(R)$ consiste das classes de isomorfismo de $R$-bimódulos centrais projetivos, finitamente gerados e de posto menor ou igual a 1 (ver [16, Proposição 3.6]). Além disso, por [16, Proposição 3.8], temos que $\mathbf{P i c S}(R)$ é um semigrupo inverso onde $[P]^{*}=\left[P^{*}\right]$, para todo $[P] \in \mathbf{P i c S}(R)$.

\section{$2.3 \quad R$-bimódulos similares}

Sejam $M$ e $N R$-bimódulos. Vamos denotar $M \mid N$ se $M$ é isomorfo, como $R$-bimódulo, a um somando direto de uma potência de $N$, ou seja, se existe um $R$-bimódulo $M^{\prime}$ tal que $N^{(n)} \simeq M \oplus M^{\prime}$, para algum $n \in \mathbb{N}$.

Essa relação satisfaz as seguintes propriedades:

(i) Reflexiva: $M \mid M$.

(ii) Transitiva: Se $M \mid N$ e $N \mid T$, então $M \mid T$.

(iii) Compatibilidade com o produto tensorial: Se $M \mid N$ e $Q$ é um $R$-bimódulo, então

$$
\left(M \otimes_{R} Q\right) \mid\left(N \otimes_{R} Q\right) \text { e }\left(Q \otimes_{R} M\right) \mid\left(Q \otimes_{R} N\right) .
$$

De fato, se $M \mid N$ então $N^{(n)} \simeq M \oplus T$, para algum $R$-bimódulo $T$ e $n \in \mathbb{N}$. Então

$$
\begin{aligned}
\left(N \otimes_{R} Q\right)^{(n)} & \simeq\left(N \otimes_{R} Q\right) \oplus\left(N \otimes_{R} Q\right) \oplus \cdots \oplus\left(N \otimes_{R} Q\right) \\
& \simeq(N \oplus N \oplus \cdots \oplus N) \otimes_{R} Q \simeq N^{(n)} \otimes_{R} Q \\
& \simeq(M \oplus T) \otimes_{R} Q \simeq\left(M \otimes_{R} Q\right) \oplus\left(T \otimes_{R} Q\right) .
\end{aligned}
$$

Logo, $\left(M \otimes_{R} Q\right) \mid\left(N \otimes_{R} Q\right)$. Analogamente, temos que $\left(Q \otimes_{R} M\right) \mid\left(Q \otimes_{R} N\right)$.

Observação 2.3.1. Sejam $M$ e $N$ dois R-bimódulos. Então $M \mid N$ se, e somente se, existem morfismos de R-bimódulos $f_{i}: M \longrightarrow N$ e $g_{i}: N \longrightarrow M$, com $i=1,2, \ldots, n$, tais que $\sum_{i=1}^{n} g_{i} \circ f_{i}=I d_{M}$. De fato, se $M \mid N$, então existem $n \in \mathbb{N}$ e um R-bimódulo $T$ tal que $N^{(n)}=M \oplus T$. Considere

$$
f_{i}: M \stackrel{\iota}{\hookrightarrow} N^{(n)} \stackrel{\pi_{i}}{\rightarrow} N \quad \text { e } \quad g_{i}: N \stackrel{\iota_{i}}{\longrightarrow} N^{(n)} \stackrel{\pi}{\rightarrow} M
$$

para $i=1,2, \ldots, n$, onde $\pi_{i}$ e $\iota_{i}$ são as projeções e injeções canônicas. Então, $f_{i}$ e $g_{i}$ são $R$-bilineares e

$$
\begin{aligned}
\sum_{i=1}^{n} g_{i} \circ f_{i} & =\sum_{i=1}^{n}\left(\pi \circ \iota_{i} \circ \pi_{i} \circ \iota\right)=\pi \circ \sum_{i=1}^{n}\left(\iota_{i} \circ \pi_{i}\right) \circ \iota \\
& =\pi \circ i d_{N^{(n)}} \circ \iota=I d_{M} .
\end{aligned}
$$

Reciprocamente, suponha que existem $f_{i}: M \longrightarrow N$ e $g_{i}: N \longrightarrow M$, com $i=1,2, \ldots, n$, tais que $\sum_{i=1}^{n} g_{i} \circ f_{i}=I d_{M}$. Considere a sequência exata

$$
1 \longrightarrow M \stackrel{f}{\longrightarrow} N^{(n)} \longrightarrow \frac{N^{(n)}}{\operatorname{Im}(f)} \longrightarrow 1
$$


onde $f: M \longrightarrow N^{(n)}$ é definida por $f(m)=\left(f_{1}(m), \ldots, f_{n}(m)\right)$. Considerando $g: N^{(n)} \longrightarrow M$ definida por

$$
g\left(x_{1}, \ldots, x_{n}\right)=\sum_{i=1}^{n} g_{i}\left(x_{i}\right)
$$

Assim, temos

$$
(g \circ f)(m)=g\left(f_{1}(m), \ldots, f_{n}(m)\right)=\sum_{i=1}^{n} g_{i}\left(f_{i}(m)\right)=m .
$$

Logo, a sequência (2.18) cinde e portanto $M$ é um somando direto de $N^{(n)}$.

Exemplo 2.3.2. (i) Se $M_{R} \mid R_{R}$ (como R-módulos à direita), então $M$ é somando direto de $R^{(n)}$, para algum $n \in \mathbb{N}$. Então, pela Proposição 2.1.2, temos que $M$ é um R-módulo projetivo e finitamente gerado à direita.

(ii) Se $R_{R} \mid M_{R}$, então $M_{R}$ é um gerador da categoria dos $R$-módulos à direita.

Observação 2.3.3. Se $M$ é um R-bimódulo tal que $M \mid R$, então $M$ é $\mathcal{Z}$-bimódulo central. De fato, pela Observação 2.3.1 existem $f_{i}: M \longrightarrow R$ e $g_{i}: R \longrightarrow M$, com $i=1,2, \ldots, n$, aplicações $R$-bilineares com $\sum_{i=1}^{n} g_{i} f_{i}=I d_{M}$. Seja $m \in P$ e $r \in \mathcal{Z}$, então

$$
m r=\sum_{i=1}^{n} g_{i}\left(f_{i}(m)\right) r=\sum_{i=1}^{n} g_{i}(\underbrace{f_{i}(m)}_{\in R} r)=\sum_{i=1}^{n} g_{i}\left(r f_{i}(m)\right)=\sum_{i=1}^{n} r g_{i}\left(f_{i}(m)\right)=r m .
$$

Portanto, $M$ é $\mathcal{Z}$-bimódulo central.

Dizemos que $M$ e $N$ são similares, e denotamos $M \sim N$, se $M \mid N$ e $N \mid M$. Claramente, $\sim$ é uma relação simétrica. Então, por (i) e (ii) acima, temos que é uma relação de equivalência.

Dado um $R$-bimódulo $M$ vamos denotar por $C_{M}(R)=\{m \in M, r m=m r$ para todo $r \in R\}$. Então, $C_{M}(R)$ é um $\mathcal{Z}$-bimódulo central.

Sobre um anel comutativo $R$, dados quaisquer dois $R$-bimódulos $M, N$ existe um isomorfismo de $R$ bimódulos $M \otimes_{R} N \simeq N \otimes_{R} M$ dado simplesmente por $m \otimes n \mapsto n \otimes m$. Para anéis não comutativos esse isomorfismo pode não existir para quaisquer $R$-bimódulos. Os próximos resultados, que se encontram em [5], nós dão uma condição para existência desse isomorfismo para $R$-bimódulos sobre um anel não comutativo. Vamos acrescentar as demonstrações desses resultados, pois em [5] as demonstrações aparecem com poucos detalhes e esses são fundamentais para a construção dos isomorfismos.

Lema 2.3.4. [5, Lema 2.4] Seja $M$ um R-bimódulo tal que $M \mid R$ (como bimódulos), então existe um isomorfismo de $R$-bimódulos $M \simeq R \otimes_{\mathcal{Z}} C_{M}(R)$, onde a estrutura de $R$-bimódulo de $R \otimes_{\mathcal{Z}} C_{M}(R)$ é dada como no Exemplo 2.1.1\%.

Demonstração. Sejam $f_{i}: M \longrightarrow R, g_{i}: R \longrightarrow M R$-bilineares, com $i=1,2, \ldots, n$, e $\sum_{i=1}^{n} g_{i} f_{i}=I d_{M}$.

Como $g_{i}$ é $R$-bilinear, então $g_{i}(1) \in C_{M}(R)$, para todo $i=1,2, \ldots, n$. Defina

$$
\begin{aligned}
\eta: M & \longrightarrow R \otimes_{\mathcal{Z}} C_{M}(R) \\
m & \longmapsto \sum_{i=1}^{n} f_{i}(m) \otimes_{\mathcal{Z}} g_{i}(1) .
\end{aligned}
$$

Claramente $\eta$ está bem definida e é $R$-bilinear. Vejamos que

$$
\begin{aligned}
\theta: R \otimes_{\mathcal{Z}} C_{M}(R) & \longrightarrow M \\
b \otimes_{\mathcal{Z}} m & \longmapsto b m
\end{aligned}
$$


é a inversa de $\eta$. Seja $m \in M$, então

$$
(\theta \circ \eta)(m)=\theta\left(\sum_{i=1}^{n} f_{i}(m) \otimes_{\mathcal{Z}} g_{i}(1)\right)=\sum_{i=1}^{n} f_{i}(m) g_{i}(1)=\sum_{i=1}^{n} g_{i}\left(f_{i}(m)\right)=m .
$$

Por outro lado, observe que se $m \in C_{M}(R)$, então $f_{i}(m) \in \mathcal{Z}$, pois

$$
r f_{i}(m)=f_{i}(r m)=f_{i}(m r)=f_{i}(m) r, \text { para todo } r \in R .
$$

Assim, dado $r \in R$ e $m \in C_{M}(R)$ temos

$$
\begin{aligned}
(\eta \circ \theta)\left(r \otimes_{\mathcal{Z}} m\right) & =\sum_{i=1}^{n} f_{i}(r m) \otimes_{\mathcal{Z}} g_{i}(1)=\sum_{i=1}^{n} r f_{i}(m) \otimes_{\mathcal{Z}} g_{i}(1) \\
& =\sum_{i=1}^{n} r \otimes_{\mathcal{Z}} f_{i}(m) g_{i}(1)=\sum_{i=1}^{n} r \otimes_{\mathcal{Z}} g_{i}\left(f_{i}(m) 1\right) \\
& =r \otimes_{\mathcal{Z}} m .
\end{aligned}
$$

Portanto, $\eta$ é um isomorfismo.

Proposição 2.3.5. [5, Corolário 3] Sejam $M$ e $N$ R-bimódulos. Se $M \mid R$ e $N \mid R$, então existe um isomorfismo de R-bimódulos

$$
\begin{aligned}
T_{M, N}: M \otimes_{R} N & \longrightarrow \otimes_{R} M \\
x \otimes y & \longmapsto \sum_{i=1}^{n} f_{i}(x) y \otimes g_{i}(1),
\end{aligned}
$$

onde $f_{i}: M \longrightarrow R, g_{i}: R \longrightarrow M$, com $i=1,2, \ldots, n$, são $R$-bilineares e satisfazem $\sum_{i=1}^{n} g_{i} f_{i}=I d_{M}$. Por outro lado, se considerarmos $f_{j}^{\prime}: N \longrightarrow R, g_{j}^{\prime}: R \longrightarrow N$, com $j=1,2, \ldots, m, R$-bilineares tais que $\sum_{j=1}^{m} g_{j}^{\prime} f_{j}^{\prime}=I d_{N}$, então o isomorfismo $T_{M, N}$ também pode ser escrito por:

$$
\begin{aligned}
T_{M, N}: M \otimes_{R} N & \longrightarrow \otimes_{R}^{N} M \\
x \otimes y & \longmapsto \sum_{j=1}^{m} g_{j}^{\prime}(1) \otimes x f_{j}^{\prime}(y) .
\end{aligned}
$$

Demonstração. O isomorfismo $T_{M, N}$ é dado pela composta

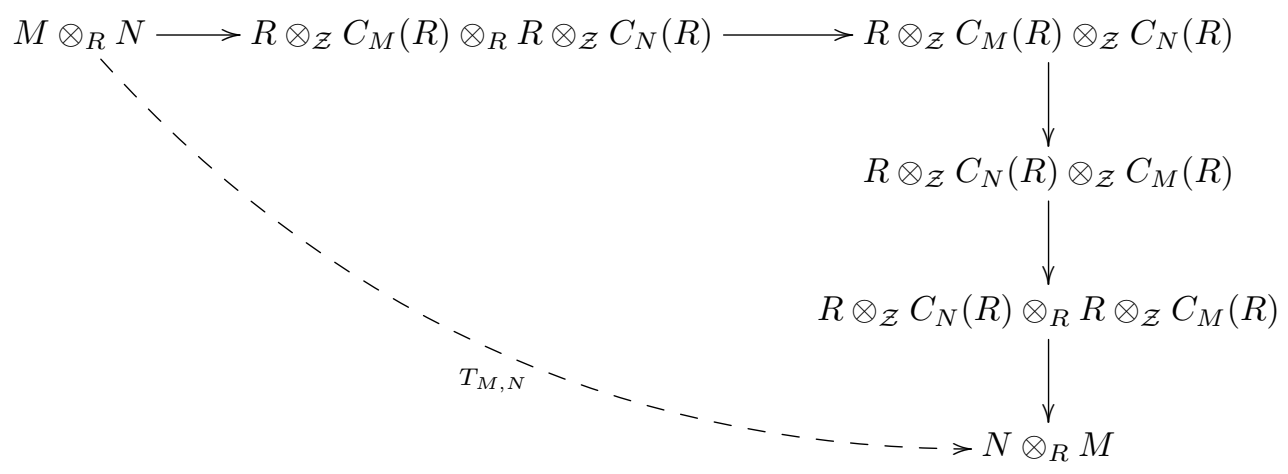


Pelo Lema 2.3.4 temos que o isomorfismo $T_{M, N}$ é dado por:

$$
\begin{aligned}
x \otimes_{R} y & \mapsto \sum_{i, j=1}^{n, m} f_{i}(x) \otimes_{\mathcal{Z}} g_{i}(1) \otimes_{R} f_{j}^{\prime}(y) \otimes_{\mathcal{Z}} g_{j}^{\prime}(1) \mapsto \sum_{i, j=1}^{n, m} f_{i}(x) f_{j}^{\prime}(y) \otimes_{\mathcal{Z}} g_{i}(1) \otimes_{\mathcal{Z}} g_{j}^{\prime}(1) \\
& \mapsto \sum_{i, j=1}^{n, m} f_{i}(x) f_{j}^{\prime}(y) \otimes_{\mathcal{Z}} g_{j}^{\prime}(1) \otimes_{\mathcal{Z}} g_{i}(1) \mapsto \sum_{i, j=1}^{n, m} f_{i}(x) f_{j}^{\prime}(y) \otimes_{\mathcal{Z}} g_{j}^{\prime}(1) \otimes_{R} 1 \otimes_{\mathcal{Z}} g_{i}(1) \\
& \stackrel{\star}{\mapsto} \sum_{i, j=1}^{n, m} f_{i}(x) f_{j}^{\prime}(y) g_{j}^{\prime}(1) \otimes_{R} g_{i}(1)=\sum_{i, j=1}^{n, m} f_{i}(x) g_{j}^{\prime}\left(f_{j}^{\prime}(y)\right) \otimes_{R} g_{i}(1) \\
& =\sum_{i=1}^{n} f_{i}(x) y \otimes_{R} g_{i}(1) .
\end{aligned}
$$

Temos portanto o isomorfismo de $R$-bimódulos

$$
\begin{aligned}
T_{M, N}: M \otimes_{R} N & \longrightarrow N_{R} M \\
x \otimes y & \longmapsto \sum_{i=1}^{n} f_{i}(x) y \otimes g_{i}(1) .
\end{aligned}
$$

Para a segunda expressão de $T_{M, N}$, basta seguir de $(\star)$ com as igualdades:

$$
\begin{aligned}
x \otimes_{R} y & \stackrel{\star}{\mapsto} \sum_{i, j=1}^{n, m} f_{i}(x) f_{j}^{\prime}(y) g_{j}^{\prime}(1) \otimes_{R} g_{i}(1) \\
& =\sum_{i, j=1}^{n, m} g_{j}^{\prime}(1) f_{i}(x) f_{j}^{\prime}(y) \otimes_{R} g_{i}(1)=\sum_{i, j=1}^{n, m} g_{j}^{\prime}(1) \otimes_{R} f_{i}(x) f_{j}^{\prime}(y) g_{i}(1) \\
& =\sum_{i, j=1}^{n, m} g_{j}^{\prime}(1) \otimes_{R} f_{i}(x) g_{i}(1) f_{j}^{\prime}(y)=\sum_{i, j=1}^{n, m} g_{j}^{\prime}(1) \otimes_{R} g_{i}\left(f_{i}(x)\right) f_{j}^{\prime}(y) \\
& =\sum_{j=1}^{m} g_{j}^{\prime}(1) \otimes_{R} x f_{j}^{\prime}(y) .
\end{aligned}
$$

Então, temos que o isomorfismo $T_{M, N}$ também pode ser escrito como

$$
T_{M, N}(x \otimes y)=\sum_{j=1}^{m} g_{j}^{\prime}(1) \otimes_{R} x f_{j}^{\prime}(y) .
$$

Corolário 2.3.6. Sejam $M$ e $N$ R-bimódulos tais que $M \mid R$ e $N$ é Z-bimódulo central. Então, existe um isomorfismo de $R$-bimódulos $M \otimes_{R} N \simeq N \otimes_{R} M$.

Demonstração. Análogo a demonstração do Lema 2.3.4 temos a cadeia de isomorfismos de $R$-bimódulos

$$
\begin{aligned}
N \otimes_{R} M & \simeq N \otimes_{R} R \otimes_{\mathcal{Z}} C_{M}(R) \simeq N \otimes_{\mathcal{Z}} C_{M}(R) \\
& \simeq C_{M}(R) \otimes_{\mathcal{Z}} N \simeq C_{M}(R) \otimes_{\mathcal{Z}} R \otimes_{R} N \\
& \simeq M \otimes_{R} N .
\end{aligned}
$$

Análogo a demonstração da Proposição 2.3.5, esse isomorfismo é dado por

$$
\begin{aligned}
T_{N, M}: N \otimes_{R} M & \longrightarrow M_{R} \otimes_{R} N \\
\otimes m & \longmapsto \sum_{i=1}^{n} g_{i}(1) \otimes n f_{i}(m),
\end{aligned}
$$


onde $f_{i}: M \longrightarrow R$ e $g_{i}: R \longrightarrow M$ são como na Observação 2.3.1.

Nos próximos exemplos vamos construir o isomorfismo $T_{-,-}$em alguns casos particulares que serão utilizados nos próximos Capítulos.

Exemplo 2.3.7. Seja e um idempotente central em $R$, então Re é um somando direto de $R$, em particular, $R e \mid R$. Se $M$ é Z्Z-bimódulo central, então existe um isomorfismo de $R$-bimódulos $R e \otimes_{R} M \simeq M \otimes_{R} R e$. Observe que a inclusão e a projeção canônica $i: R e \longrightarrow R$ e $\pi: R \longrightarrow$ Re satisfazem as condições da Observação 2.3.1, então temos os isomorfismos

$$
\begin{aligned}
& R e \otimes_{R} M \quad \longrightarrow M \otimes_{R} R e \quad \otimes_{e} \quad M \otimes_{R} R e \longrightarrow R e \otimes_{R} M \\
& r e \otimes m \longrightarrow r m \otimes e \quad e \quad m \otimes r e \longrightarrow e \otimes m r
\end{aligned}
$$

Exemplo 2.3.8. Sejam $M$ e $N$ R-bimódulos tais que $N \mid R$ e existe um isomorfismo de $R$-bimódulos $f: M \longrightarrow R$. Em particular, $M \mid R$. Observe que o isomorfismo de R-bimódulos $f: M \longrightarrow R$ e sua inversa $f^{-1}: R \longrightarrow M$ satisfazem a condição da Observação 2.3.1, então o isomorfismo $T_{M, N}$ pode ser escrito como

$$
\begin{aligned}
T_{M, N}: M \otimes_{R} N & \longrightarrow N \otimes_{R} M \\
m \otimes n & \longmapsto f(m) n \otimes f^{-1}(1)
\end{aligned}
$$

\subsection{O grupo $\mathcal{P}(S / R)$}

Sejam $R \subseteq S$ uma extensão de anéis com mesma unidade. Vamos denotar $\mathcal{M}(S / R)$ o conjunto dos elementos $P=[\phi] \Longrightarrow X$, onde $P$ um $R$-bimódulo, $X$ um $S$-bimódulo e $\phi: P \longrightarrow X$ uma aplicação $R$-bilinear tal que as aplicações

$$
\begin{aligned}
\bar{\phi}_{r}: P \otimes_{R} S & \longrightarrow X \\
p \otimes_{R} s & \longrightarrow \phi(p) s
\end{aligned} \quad \text { e } \quad \begin{aligned}
\bar{\phi}_{l}: S \otimes_{R} P & \longrightarrow X \\
&
\end{aligned} \quad \begin{array}{ll}
\otimes_{R} p & \longrightarrow s \phi(p)
\end{array}
$$

são isomorfismos de $R$-S-bimódulos e $S$ - $R$-bimódulos, respectivamente. Dados $P=[\phi] \Longrightarrow$ e $Q=[\psi] \Longrightarrow Y$ em $\mathcal{M}(S / R)$, definimos o elemento $P \otimes_{R} Q=[\phi \otimes \psi] \Longrightarrow X \otimes_{S} Y$, onde

$$
\begin{aligned}
\phi \otimes \psi: P \otimes_{R} Q & \longrightarrow X \otimes_{S} Y \\
p \otimes q & \longmapsto \phi(p) \otimes \psi(q) .
\end{aligned}
$$

Afirmação 2.4.1. $\left[P \otimes_{R} Q\right]=[\phi \otimes \psi] \Longrightarrow\left[X \otimes_{S} Y\right] \in \mathcal{M}(S / R)$.

De fato, temos o isomorfismo de $S$ - $R$-bimódulos

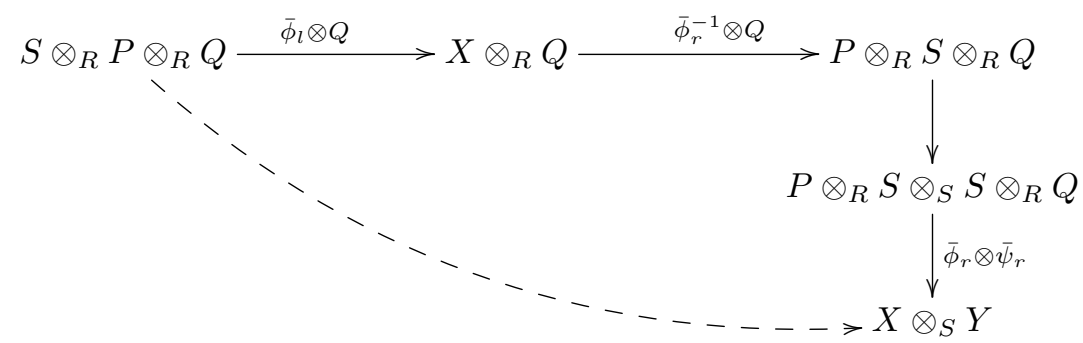

Observe agora que dado $p \in P, q \in Q$ e $s \in S$, então

$$
\begin{aligned}
s \otimes p \otimes q & \mapsto s \phi(p) \otimes q \mapsto \bar{\phi}_{r}^{-1}(s \phi(p)) \otimes q \\
& \mapsto \bar{\phi}_{r}^{-1}(s \phi(p)) \otimes_{S} 1 \otimes q \mapsto s \phi(p) \otimes_{S} \psi(q) \\
& =\frac{{ }_{(\phi \otimes \psi)}}{(s \otimes p \otimes q) .}
\end{aligned}
$$


Logo, $\overline{(\phi \otimes \psi)_{l}}$ é isomorfismo de $S$ - $R$-bimódulo. Analogamente mostra-se que $\overline{(\phi \otimes \psi)_{r}}$ é isomorfismo de $R$-S-bimodulos. Essa operação é claramente associativa e $R=[\iota] \Longrightarrow S$, onde $\iota$ denota a inclusão de $R$ em $S$, é o elemento neutro.

Um morfismo entre $P=[\phi] \Longrightarrow X$ e $Q=[\psi] \Longrightarrow Y$ é um par $(\alpha, \beta)$, onde $\alpha: P \longrightarrow Q$ é $R$-bilinear, $\beta: X \longrightarrow Y$ é $S$-bilinear e o diagrama

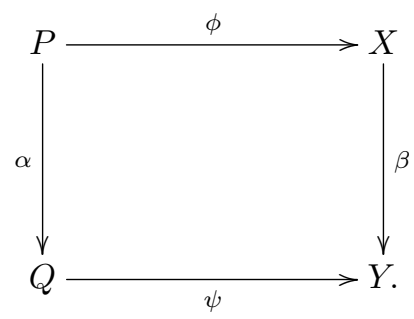

é comutativo. Se $\alpha$ e $\beta$ são isomorfismos de $R$-bimódulos e $S$-bimódulos, respectivamente, então $(\alpha, \beta)$ é um isomorfismo entre $P=[\phi] \Longrightarrow X$ e $Q=[\psi] \Longrightarrow Y$.

Vamos denotar por $\mathcal{P}(S / R)$ o conjunto das classes de isomorfismos dos elementos $[P]=[\phi] \Longrightarrow[X]$, onde $[P] \in \mathbf{P i c}(R)$ e $[X] \in \mathbf{P i c}(S)$.

Proposição 2.4.2. Seja $R \subseteq S$ uma extensão de anéis com mesma unidade. Considere $[P] \in \mathbf{P i c}(R)$, $[X] \in \operatorname{Pic}(S)$ e $\phi: P \longrightarrow X$ uma aplicação R-bilinear. Suponha que $\bar{\phi}_{l}$ é isomorfismo. Então:

i) $\bar{\phi}_{r}$ é isomorfismo.

ii) $\phi$ é injetora.

iii) $\left[P^{*}\right]=\left[\phi^{*}\right] \Rightarrow\left[X^{*}\right] \in \mathcal{P}(S / R)$, onde $\phi^{*}(f)(s \phi(p))=s f(p)$, para $s \in S$ e $p \in P$.

iv) $\left[{ }^{*} P\right]=\left[\phi^{+}\right] \Rightarrow\left[X^{*}\right] \in \mathcal{P}(S / R)$, onde $\phi^{+}(g)(\phi(p) s)=g(p) s$, para $s \in S$ e $p \in P$.

v) $\left(\left[P^{*}\right]=\left[\phi^{*}\right] \Rightarrow\left[X^{*}\right]\right)=\left(\left[{ }^{*} P\right]=\left[\phi^{+}\right] \Rightarrow\left[{ }^{*} X\right]\right)$ em $\mathcal{P}(S / R)$.

Demonstração. [18, Lema 3.1].

Observação 2.4.3. (i) Na Proposição 2.4.2, se supormos que $\bar{\phi}_{r}$ é isomorfismo, ao invés de $\bar{\phi}_{l}$, também chega-se a conclusão que $\bar{\phi}_{l}$ é isomorfismo e $\phi$ injetora.

(ii) Segue Proposição 2.4.2 que se $[P] \in \mathbf{P i c}(R)$ e $[X] \in \mathbf{P i c}(S)$, então $[P] \Longrightarrow[\phi] \Longrightarrow[X] \in \mathcal{P}(S / R)$ se $\bar{\phi}_{l}$ (ou $\left.\bar{\phi}_{r}\right)$ é isomorfismo.

Proposição 2.4.4. $\mathcal{P}(S / R)$ é um grupo.

Demonstração. Basta mostrar que cada elementos em $\mathcal{P}(S / R)$ possui um inverso. Dado $[P]=[\phi] \Longrightarrow[X] \in$ $\mathcal{P}(S / R)$, pela Proposição 2.4.2, $\left[P^{*}\right]=\left[\phi^{*}\right] \Rightarrow\left[X^{*}\right]$ também pertence a $\mathcal{P}(S / R)$. É fácil ver que o dia- 
grama abaixo é comutativo

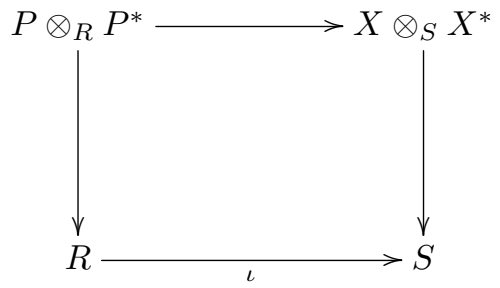

onde os isomorfismos na vertical são dados como na Observação 2.1.13. Logo, $\left[P^{*}\right]=\left[\phi^{*}\right] \Rightarrow\left[X^{*}\right]$ é o inverso de $[P]=[\phi] \Longrightarrow[X]$ em $\mathcal{P}(S / R)$.

Se $R$ é um $K$-álgebra definimos $\mathcal{P}_{K}(S / R)=\left\{[P]=[\phi] \Longrightarrow[X] \in \mathcal{P}(S / R) ;[P] \in \mathbf{P i c}_{K}(R)\right\}$. É fácil ver que $\mathcal{P}_{K}(S / R)$ é um subgrupo de $\mathcal{P}(S / R)$. De fato, basta ver que se $P$ é $K$-bimódulo central, então $P^{*}$ também é. Sejam $k \in K$ e $f \in P^{*}$, então

$$
(f \cdot k)(p)=f(p) k=k f(p)=f(k p)=f(p k)=(k \cdot f)(p),
$$

para todo $p \in P$. Portanto, $\left[P^{*}\right] \in \mathbf{P i c}_{K}(R)$.

\subsection{Uma sequência exata auxiliar}

Nesta seção vamos apresentar a sequência exata que foi construída em [5, Teorema 1.5]. Essa sequência será usada na construção da nossa sequência no Capítulo 4. Vamos colocar a demonstração completa da exatidão já que na prova apresentada em [5] são omitidos detalhes importantes que serão usados mais adiante.

Seja $R \subseteq S$ uma extensão de anéis com mesma unidade. Vamos denotar por $\operatorname{Aut}_{R-\text { rings }}(S)$ o grupo dos automorfismos do anel $S$ que fixam cada elemento de $R$. Então,

$$
\begin{aligned}
\mathcal{F}: \mathcal{U}(\mathcal{Z}) & \longrightarrow \text { Aut }_{R-\text { rings }}(S) \\
r & \longmapsto\left(s \mapsto r s r^{-1}\right)
\end{aligned}
$$

é um morfismo de grupos.

Dado $f \in \mathbf{A u t}_{R \text {-rings }}(S)$, definimos o $S$-bimódulo $S_{f}$ por: $S_{f}=S$, como grupos, e ações dadas por:

$$
s^{\prime} * t=s^{\prime} t \text { e } t * s=t f(s), \text { para } s, s^{\prime} \in S, t \in S_{f} .
$$

Então,

$$
\begin{aligned}
\mathcal{E}: \operatorname{Aut}_{R-\operatorname{ringS}}(S) & \longrightarrow \mathcal{P}(S / R) \\
f & \longmapsto\left([R]=\left[\iota_{f}\right] \Longrightarrow\left[S_{f}\right]\right),
\end{aligned}
$$

onde $\iota_{f}: R \longrightarrow S_{f}$ é a inclusão, é um morfismo de grupos.

Teorema 2.5.1. A sequência

$$
\mathcal{U}(\mathcal{Z}) \stackrel{\mathcal{F}}{\longrightarrow} \operatorname{Aut}_{R-\operatorname{rings}}(S) \stackrel{\mathcal{E}}{\longrightarrow} \mathcal{P}(S / R) \stackrel{\vartheta}{\longrightarrow} \mathbf{P i c}(R),
$$

onde $\vartheta([P]=[\phi] \Longrightarrow[X])=[P]$, é exata. 
Demonstração. Vejamos a exatidão em $\operatorname{Aut}_{R \text {-rings }}(S)$. Seja $f \in \mathbf{A u t}_{R \text {-rings }}(S)$ tal que $\left([R]=\left[\iota_{f}\right] \Longrightarrow\left[S_{f}\right]\right)=$ $([R]=[\iota] \Longrightarrow[S])$ em $\mathcal{P}(S / R)$. Temos então o diagrama comutativo

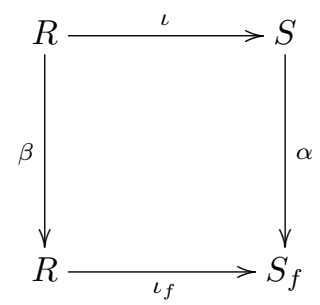

onde $\alpha$ é um isomorfismo de $S$-bimódulos. Pela comutatividade do diagrama, temos que $R=\alpha(R)$. Seja $d=\alpha^{-1}(1) \in R$. Por $\alpha$ ser isomorfismo de $S$-bimódulos e $f(r)=r$, para todo $r \in R$, temos que $d \in \mathcal{U}(\mathcal{Z})$. Além disso,

$$
d f(s)=d * s=\alpha^{-1}(1) * s=\alpha^{-1}(s)=s \alpha^{-1}(1)=s d,
$$

para todo $s \in S$. Logo, $f(s)=d^{-1} s d=\mathcal{F}\left(d^{-1}\right)(s)$, para todo $s \in S$. Ou seja, $\mathcal{F}\left(d^{-1}\right)=f$. Logo, $f \in \operatorname{Im}(\mathcal{F})$.

Seja $f \in \operatorname{Im}(\mathcal{F})$, então existe $r \in \mathcal{U}(\mathcal{Z})$ tal que $f(s)=r s r^{-1}$, para todo $s \in S$. Seja $\omega: S \longrightarrow S_{f}$ definido por $\omega(s)=s r^{-1}$. Claramente, $\omega$ é isomorfismo e $S$-linear à esquerda. Dados $s, s^{\prime} \in S$, temos

$$
\omega\left(s s^{\prime}\right)=s s^{\prime} r^{-1}=s r^{-1} r s^{\prime} r^{-1}=s r^{-1} f\left(s^{\prime}\right)=\omega(s) * s^{\prime} .
$$

Portanto, $\omega$ é isomorfismo de $S$-bimódulos. Considere o isomorfismo de $R$-bimódulos $\lambda: R \longrightarrow R$, definido por $\lambda(x)=x r^{-1}$. Então, o diagrama

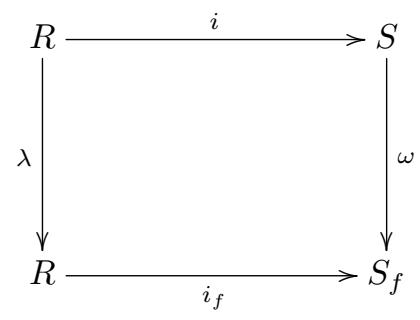

é comutativo. Logo, $\mathcal{E}(f)=\left([R]=\left[\iota_{f}\right] \Rightarrow\left[S_{f}\right]\right)=([R]=[l] \Longrightarrow[S])$ em $\mathcal{P}(S / R)$. Portanto, $f \in \operatorname{ker}(\mathcal{E})$.

Para a exatidão em $\mathcal{P}(S / R)$, considere $([P]=[\phi] \Longrightarrow[X]) \in \mathcal{P}(S / R)$ com $[P]=[R]$ em $\mathbf{P i c}(R)$. Assim existe um isomorfismo $R$-bilinear $\gamma: R \longrightarrow P$. Então, temos os seguintes isomorfismos de $(R, S)$ bimódulos e $(S, R)$-bimódulo

$$
\begin{aligned}
& \alpha: S \stackrel{\simeq}{\longrightarrow} R \otimes_{R} S \stackrel{\gamma \otimes_{R} S}{\longrightarrow} P \otimes_{R} S \stackrel{\bar{\phi}_{r}}{\longrightarrow} X \\
& \beta: S \stackrel{\simeq}{\longrightarrow} S \otimes_{R} R \stackrel{S \otimes_{R} \gamma}{\longrightarrow} S \otimes_{R} P \stackrel{\bar{\phi}_{l}}{\longrightarrow} X
\end{aligned}
$$

Observe que $\alpha(s)=\phi(\gamma(1)) s$ e $\beta(s)=s \phi(\gamma(1))$, para $s \in S$. Defina $f=\beta^{-1} \circ \alpha: S \longrightarrow S$. Então, por definição temos

$$
f(s) \phi(\gamma(1))=\beta(f(s))=\alpha(s)=\phi(\gamma(1)) s,
$$

para todo $s \in S$. Então, para $s, t \in S$ temos

$$
\begin{aligned}
\alpha(s t) & =\phi(\gamma(1)) s t \stackrel{(2.20)}{=} f(s) \phi(\gamma(1)) t \\
\stackrel{(2.20)}{=} & f(s) f(t) \phi(\gamma(1)) \\
& =\beta(f(s) f(t)) .
\end{aligned}
$$


Aplicando $\beta^{-1}$ temos que $f(s t)=f(s) f(t)$. Além disso, como $\phi$ e $\gamma$ são $R$-bilineares temos que

$$
\alpha(r)=\phi(\gamma(1)) r=\phi(\gamma(r))=r \phi(\gamma(1))=\beta(r),
$$

para todo $r \in R$. Aplicando $\beta^{-1}$ temos que $f(r)=r$, para todo $r \in R$. Logo, $f \in \operatorname{Aut}_{R \text {-rings }}(S)$. Considere $\beta: S_{f} \longrightarrow X$. Claramente $\beta$ é $S$-linear à esquerda. Dado $t \in S$ e $s \in S_{f}$ temos

$$
\begin{array}{ccl}
\beta(s * t) & = & \beta(s f(t))=s f(t) \phi(\gamma(1)) \\
\stackrel{(2.20)}{=} & s \phi(\gamma(1)) t=\beta(s) t .
\end{array}
$$

Logo, $\beta: S_{f} \longrightarrow X$ é um isomorfismo $S$-bilinear. Além disso, por $\beta(r)=(\phi \circ \gamma)(r)$, para todo $r \in R$, temos que o diagrama abaixo é comutativo

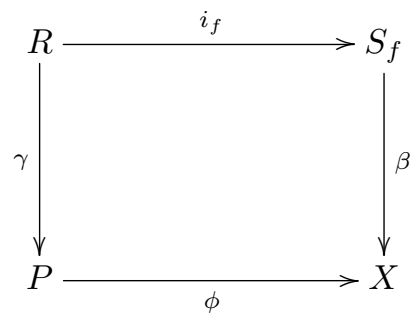

Assim, $\mathcal{E}(f)=\left([R]=\left[\iota_{f}\right] \Rightarrow\left[S_{f}\right]\right)=([P]=[\phi] \Longrightarrow[X])$ em $\mathcal{P}(S / R)$. Logo, $\operatorname{ker}(\vartheta) \subseteq \operatorname{Im}(\mathcal{E})$. A inclusão contrária é imediata. 


\section{Capítulo 3}

\section{Fundamentos sobre ações e representações parciais}

Neste Capítulo vamos construir as ações parciais que serão usadas na construção dos grupos da sequência. Para cada representação parcial unital (ver Seção 3.2 ) de $G$ em $\mathbf{P i c S}(R)$ temos uma ação parcial sobre o centro do anel, que será denotado por $\mathcal{Z}$, e sobre $\mathbf{P i c S}_{\mathcal{Z}}(R)$ (subseção 3.2.1). Na Seção 3.3 definimos o produto cruzado generalizado parcial que também é construído a partir de uma representação parcial unital de $G$ em $\mathbf{P i c S}(R)$. Esse conceito foi introduzido em [17] para uma extensão de Galois parcial de anéis comutativos. Na subseção 3.3.1, relacionamos o produto cruzado generalizado parcial com uma extensão de anéis $R \subseteq S$ com mesma unidade. Na ultima seção desse capítulo, construímos o grupo $\mathcal{C}(\Theta / R)$, para uma representação parcial unital $\Theta: G \longrightarrow \operatorname{PicS}(R)$ fixada, e o subgrupo $\mathcal{C}_{0}(\Theta / R)$.

Vamos começar relembrando na Seção 3.1 o conceito de ações parciais, representações parciais e cohomologia parcial que podem ser vistos com mais detalhes em [13], [26] e [15].

A menos de menção contrária, todo os tensores serão tomados sobre o anel $R$.

\subsection{Ações parciais, representações parciais e cohomologia par- cial.}

Definição 3.1.1. Sejam $G$ um grupo e $S$ um semigrupo. Uma ação parcial de $G$ sobre $S$ é uma coleção de subsemigrupos $S_{x}$, com $x \in G$, e isomorfismos de semigrupos $\alpha_{x}: S_{x^{-1}} \longrightarrow S_{x}$ que satisfazem as condições:

(i) $S_{1}=S$ e $\alpha_{1}=I d_{S}$,

(ii) $\alpha_{y}^{-1}\left(S_{y} \cap S_{x^{-1}}\right) \subseteq S_{(x y)^{-1}}$,

(iii) $\alpha_{x} \circ \alpha_{y}(s)=\alpha_{x y}(s)$, para cada $s \in \alpha_{y}^{-1}\left(S_{y} \cap S_{x^{-1}}\right)$.

Vamos denotar por $\alpha=\left(S_{x}, \alpha_{x}\right)$ uma ação parcial de $G$ sobre $S$, onde $S_{x}$ são os subsemigrupos e $\alpha_{x}$ são os isomorfismos. Como visto em [13], as condições (ii) e (iii) da definição acima implicam que $\alpha_{x}^{-1}=\alpha_{x^{-1}} \mathrm{e}$

$$
\alpha_{x}\left(S_{x^{-1}} \cap S_{y}\right)=S_{x} \cap S_{x y}, \quad \text { para todo } x, y \in G .
$$


Se cada $S_{x}$ é gerado por um idempotente central de $S$, ou seja, $S_{x}=S 1_{x}$, para todo $x \in G$, então $S_{x} \cap S_{y}=S 1_{x} 1_{y}$ e por (3.1) temos $\alpha_{x}\left(1_{y} 1_{x^{-1}}\right)=1_{x} 1_{x y}$, para todo $x, y \in G$. Como uma consequência dessa igualdade temos:

$$
\alpha_{x y}\left(s 1_{y^{-1} x^{-1}}\right) 1_{x}=\alpha_{x}\left(\alpha_{y}\left(s 1_{y^{-1}}\right) 1_{x^{-1}}\right), \text { para todo } x, y \in G \text { r } s \in S .
$$

De fato,

$$
\begin{aligned}
\alpha_{x}\left(\alpha_{y}\left(s 1_{y^{-1}}\right) 1_{x^{-1}}\right) & =\alpha_{x}\left(\alpha_{y}\left(s 1_{y^{-1}}\right) 1_{y} 1_{x^{-1}}\right)=\alpha_{x}\left(\alpha_{y}\left(s 1_{y^{-1}}\right) \alpha_{y}\left(1_{y^{-1}} 1_{y^{-1} x^{-1}}\right)\right) \\
& =\alpha_{x}\left(\alpha_{y}\left(s 1_{y^{-1}} 1_{y^{-1}} 1_{y^{-1} x^{-1}}\right)\right)=\alpha_{x}\left(\alpha_{y}\left(s 1_{y^{-1}} 1_{y^{-1} x^{-1}}\right)\right) \\
& =\alpha_{x y}\left(s 1_{y^{-1}} 1_{y^{-1} x^{-1}}\right)=\alpha_{x y}\left(s 1_{y^{-1} x^{-1}} 1_{y^{-1}} 1_{y^{-1} x^{-1}}\right) \\
& =\alpha_{x y}\left(s 1_{y^{-1} x^{-1}}\right) \alpha_{x y}\left(1_{y^{-1}} 1_{y^{-1} x^{-1}}\right) \\
& =\alpha_{x y}\left(s 1_{y^{-1} x^{-1}}\right) 1_{x y} 1_{x} \\
& =\alpha_{x y}\left(s 1_{y^{-1} x^{-1}}\right) 1_{x} .
\end{aligned}
$$

O subconjunto dos invariantes de $S$ sobre $\alpha$ é definido por

$$
S^{\alpha}=\left\{s \in S ; \alpha_{x}\left(s 1_{x^{-1}}\right)=s 1_{x}, \text { para todo } x \in G\right\} .
$$

Definição 3.1.2. Uma representação parcial de $G$ em um monoide $S$ é uma aplicação

$$
\begin{aligned}
\theta: G & \longrightarrow S \\
x & \longmapsto \theta_{x}
\end{aligned}
$$

que satisfaz as seguintes propriedades:

(i) $\theta_{1_{G}}=1_{S}$,

(ii) $\theta_{x} \theta_{y} \theta_{y^{-1}}=\theta_{x y} \theta_{y^{-1}}$, para todos $x, y \in G$.

(iii) $\theta_{x^{-1}} \theta_{x} \theta_{y}=\theta_{x^{-1}} \theta_{x y}$, para todos $x, y \in G$.

Segue diretamente da definição que $\theta_{x} \theta_{x^{-1}} \theta_{x}=\theta_{x}$, para todo $x \in G$. Para cada $x \in G$ denote $\varepsilon_{x}=\theta_{x} \theta_{x^{-1}}$. Por [26] temos que $\varepsilon_{x}^{\prime} s$ são idempotentes que comutam e satisfazem

$$
\theta_{x} \varepsilon_{y}=\varepsilon_{x y} \theta_{x} \text { para todos } x, y \in G .
$$

Em particular,

$$
\varepsilon_{x} \theta_{x}=\theta_{x} \quad \text { e } \quad \theta_{x} \varepsilon_{x^{-1}}=\theta_{x}, \text { para todo } x \in G .
$$

Segue da definição que

$$
\theta_{x} \theta_{y}=\theta_{x} \theta_{x^{-1}} \theta_{x} \theta_{y}=\theta_{x} \theta_{x^{-1}} \theta_{x y}=\varepsilon_{x} \theta_{x y}, \quad \text { para todos } x, y \in G .
$$

Aplicando (3.2) a (3.4) temos

$$
\theta_{x} \theta_{y}=\varepsilon_{x} \theta_{x y}=\theta_{x y} \varepsilon_{y^{-1}}, \text { para todos } x, y \in G .
$$

Proposição 3.1.3. Sejam $S$ um monoide e $\theta: G \longrightarrow S$ uma representação parcial. Considere o subsemigrupo $S_{x}=\varepsilon_{x} S \varepsilon_{x}$, para $x \in G$. Então, temos uma ação parcial $\alpha^{*}=\left(S_{x}, \alpha_{x}^{*}\right)$, onde

$$
\begin{aligned}
\alpha_{x}^{*}: S_{x^{-1}} & \longrightarrow S_{x} \\
s & \longmapsto \theta_{x} s \theta_{x^{-1}} .
\end{aligned}
$$


Demonstração. Claramente $S_{1}=S$ e $\alpha_{1}^{*}=I d_{S}$. Observe que $s \in S_{x}$ se, e somente se, $\varepsilon_{x} s \varepsilon_{x}=s$. De fato, claramente se $s=\varepsilon_{x} s \varepsilon_{x}$, então $s \in S_{x}$. Por outro lado, se $s \in S_{x}$, então $s=\varepsilon_{x} s^{\prime} \varepsilon_{x}$, para algum $s^{\prime} \in S$, então $\varepsilon_{x} s \varepsilon_{x}=\varepsilon_{x} \varepsilon_{x} s^{\prime} \varepsilon_{x} \varepsilon_{x}=\varepsilon_{x} s^{\prime} \varepsilon_{x}=s$. O que mostra a afirmação.

Por (3.3), temos

$$
\alpha_{x}^{*}(s)=\theta_{x} s \theta_{x^{-1}}=\varepsilon_{x} \theta_{x} s \theta_{x^{-1}} \varepsilon_{x}=\varepsilon_{x} \alpha_{x}^{*}(s) \varepsilon_{x} .
$$

Assim, $\alpha_{x}^{*}(s) \in S_{x}$. Dados $s, s^{\prime} \in S_{x^{-1}}$, temos

$$
\alpha_{x}^{*}\left(s s^{\prime}\right)=\theta_{x} s s^{\prime} \theta_{x^{-1}}=\theta_{x} s \varepsilon_{x^{-1}} s^{\prime} \theta_{x^{-1}}=\theta_{x} s \theta_{x^{-1}} \theta_{x} s^{\prime} \theta_{x^{-1}}=\alpha_{x}^{*}(s) \alpha_{x}^{*}\left(s^{\prime}\right) .
$$

Logo, $\alpha_{x}^{*}$ é um morfismo de semigrupos. Considere $\alpha_{x^{-1}}^{*}: S_{x} \longrightarrow S_{x^{-1}}$. Dado $s \in S_{x^{-1}}$ temos

$$
\begin{aligned}
\left(\alpha_{x^{-1}}^{*} \circ \alpha_{x}^{*}\right)(s) & =\alpha_{x^{-1}}^{*}\left(\theta_{x} s \theta_{x^{-1}}\right)=\theta_{x^{-1}} \theta_{x} s \theta_{x^{-1}} \theta_{x} \\
& =\varepsilon_{x^{-1}} s \varepsilon_{x^{-1}}=s,
\end{aligned}
$$

pois $s \in S_{x^{-1}}$. Analogamente, $\left(\alpha_{x}^{*} \circ \alpha_{x^{-1}}^{*}\right)(s)=s$, para todo $s \in S_{x}$. Então, $\alpha_{x}^{*}$ é isomorfismo com inverso $\alpha_{x^{-1}}^{*}$.

Seja $s \in S_{y} \cap S_{x^{-1}}$, então

$$
\begin{aligned}
& \alpha_{y^{-1}}^{*}(s)=\theta_{y^{-1}} s \theta_{y} \stackrel{s \in S_{x}-1}{=} \theta_{y^{-1}} \varepsilon_{x^{-1}} s \varepsilon_{x^{-1}} \theta_{y} \\
& \stackrel{(3.2)}{=} \\
& \varepsilon_{(x y)^{-1}} \theta_{y^{-1}} s \theta_{y} \varepsilon_{(x y)^{-1}} \in S_{(x y)^{-1}} .
\end{aligned}
$$

Logo, temos $\alpha_{y^{-1}}^{*}\left(S_{y} \cap S_{x^{-1}}\right) \subseteq S_{(x y)^{-1}}$. Seja $s \in \alpha_{y^{-1}}^{*}\left(S_{y} \cap S_{x^{-1}}\right) \subseteq S_{(x y)^{-1}} \cap S_{y^{-1}}$, por (3.4) e (3.5) temos:

$$
\begin{aligned}
\left(\alpha_{x}^{*} \circ \alpha_{y}^{*}\right)(s) & =\alpha_{x}^{*}\left(\theta_{y} s \theta_{y^{-1}}\right)=\theta_{x} \theta_{y} s \theta_{y^{-1}} \theta_{x^{-1}} \\
& =\theta_{x y} \varepsilon_{y^{-1}} s \varepsilon_{y^{-1}} \theta_{y^{-1} x^{-1}} \\
& =\theta_{x y} s \theta_{y^{-1} x^{-1}} \\
& =\alpha_{x y}^{*}(s) .
\end{aligned}
$$

Portanto, $\alpha^{*}$ é uma ação parcial.

A noção de cohomologia parcial foi introduzida em [15]. Sejam $K$ um anel (ou um monoide) comutativo e $\alpha=\left(D_{x}, \alpha_{x}\right)$ uma ação parcial unitária de $G$ sobre $K$, onde cada $D_{x}$ é um ideal de $K$ gerado por um idempotente central $1_{x}$. Uma $n$-cocadeia, com $n \in \mathbb{N}$, de $G$ com valores em $K$ é uma função $f: G^{n} \longrightarrow K$ tal que $f\left(x_{1}, \ldots, x_{n}\right) \in \mathcal{U}\left(K 1_{x} 1_{x_{1} x_{2}} \ldots 1_{x_{1} x_{2} \ldots x_{n}}\right)$. Uma 0 -cocadeia é definida como sendo um elemento em $\mathcal{U}(K)$.

Seja $C^{n}(G, \alpha, K)$ o conjunto de todas as $n$-cocadeias de $G$ com valores em $K$. Considere em $C^{n}(G, \alpha, K)$ a multiplicação definida ponto a ponto, ou seja, se $f, g \in C^{n}(G, \alpha, K)$, então

$$
f g\left(x_{1}, \ldots, x_{n}\right)=f\left(x_{1}, \ldots, x_{n}\right) g\left(x_{1}, \ldots, x_{n}\right), \quad \text { para } x_{1}, \ldots, x_{n} \in G .
$$

Então, $C^{n}(G, \alpha, K)$ é um grupo, onde o elemento neutro é $I\left(x_{1}, \ldots, x_{n}\right)=1_{x_{1}} 1_{x_{1} x_{2} \ldots 1_{x_{1} x_{2} \ldots x_{n}}}$ e $f^{-1}\left(x_{1}, \ldots, x_{n}\right)=$ $f\left(x_{1}, \ldots, x_{n}\right)^{-1}$, para $x_{1}, \ldots, x_{n} \in G$.

Seja $f \in C^{n}(G, \alpha, K)$ e $x_{1}, \ldots, x_{n}, x_{n+1} \in G$. Defina

$$
\begin{aligned}
\left(\delta^{n} f\right)\left(x_{1}, \ldots, x_{n+1}\right)= & \alpha_{x_{1}}\left(f\left(x_{2}, \ldots, x_{n+1}\right) 1_{x_{1}^{-1}}\right) \prod_{i=1}^{n} f\left(x_{1}, \ldots, x_{i} x_{i+1}, \ldots, x_{n+1}\right)^{(-1)^{i}} \\
& f\left(x_{1}, \ldots, x_{n}\right)^{(-1)^{n+1}} .
\end{aligned}
$$


Para $n=0$ definimos

$$
\left(\delta^{0} k\right)(x)=\alpha_{x}\left(k 1_{x^{-1}}\right) k^{-1}, \quad \text { onde } k \in \mathcal{U}(K) .
$$

Proposição 3.1.4. A aplicação $\delta^{n}: C^{n}(G, \alpha, K) \longrightarrow C^{n+1}(G, \alpha, K)$ definida em (3.6) é um morfismo de grupos tal que

$$
\left(\delta^{n+1} \circ \delta^{n}\right)(f)\left(x_{1}, \ldots, x_{n+2}\right)=1_{x_{1}} 1_{x_{1} x_{2}} \ldots 1_{x_{1} x_{2} \ldots x_{n+2}},
$$

para todo $f \in C^{n}(G, \alpha, K)$ e $x_{1}, \ldots, x_{n+1} \in G$.

Demonstração. [15, Proposição 1.5].

Como no caso clássico, vamos definir

$$
Z^{n}(G, \alpha, K)=\operatorname{ker}\left(\delta^{n}\right) \text { e } B^{n}(G, \alpha, K)=\operatorname{Im}\left(\delta^{n-1}\right)
$$

que são chamados de $n$-cociclos e $n$-cobordos parciais. Pela Proposição 3.1.4, temos que $B^{n}(G, \alpha, K) \subseteq$ $Z^{n}(G, \alpha, K)$. Definimos então o grupo $n$-cohomologia parcial por

$$
H^{n}(G, \alpha, K)=\frac{Z^{n}(G, \alpha, K)}{B^{n}(G, \alpha, K)} .
$$

Para $n=0$ definimos $H^{0}(G, \alpha, K)=Z^{0}(G, \alpha, K)=\operatorname{ker}\left(\delta^{0}\right)$.

No próximo exemplo vamos descrever o morfismo $\delta^{n}$ e os grupos $Z^{n}(G, \alpha, K)$ e $B^{n}(G, \alpha, K)$, para $n=0,1,2$.

Exemplo 3.1.5. Para $n=0$ temos

$$
\begin{gathered}
H^{0}(G, \alpha, \mathcal{Z})=Z^{0}(G, \alpha, \mathcal{Z})=\left\{k \in \mathcal{U}(K), \alpha_{x}\left(k 1_{x^{-1}}\right)=k 1_{x}, \forall x \in G\right\} \\
B^{1}(G, \alpha, K)=\operatorname{Im}\left(\delta^{0}\right)=\left\{f \in C^{1}(G, \alpha, K) ; \exists k \in \mathcal{U}(K) \quad f(x)=\alpha_{x}\left(k 1_{x^{-1}}\right) k^{-1}, \forall x \in G\right\} .
\end{gathered}
$$

Para $n=1$, temos $\left(\delta^{1} f\right)(x, y)=\alpha_{x}\left(f(y) 1_{x^{-1}}\right) f(x y)^{-1} f(x)$, para $f \in C^{1}(G, \alpha, K)$. Então,

$$
Z^{1}(G, \alpha, K)=\left\{f \in C^{1}(G, \alpha, K) ; \alpha_{x}\left(f(y) 1_{x^{-1}}\right) f(x)=f(x y) 1_{x}, \forall x, y \in G\right\} .
$$

$B^{2}(G, \alpha, K)=\left\{f \in C^{2}(G, \alpha, K) ; \exists \sigma \in C^{1}(G, \alpha, K), \operatorname{com} f(x, y)=\alpha_{x}\left(\sigma(y) 1_{x^{-1}}\right) \sigma(x y)^{-1} \sigma(x), \forall x, y \in G\right\}$.

Para $n=2$,

$$
\left(\delta^{2} f\right)(x, y, z)=\alpha_{x}\left(f(y, z) 1_{x^{-1}}\right) f(x y, z)^{-1} f(x, y z) f(x, y)^{-1},
$$

para $f \in C^{2}(G, \alpha, K)$ e $x, y \in G$. Então,

$$
Z^{2}(G, \alpha, G)=\left\{f \in C^{2}(G, \alpha, K) ; \alpha_{x}\left(f(y, z) 1_{x^{-1}}\right) f(x, y z)=f(x y, z) f(x, y), \forall x, y, z \in G\right\} .
$$

Sejam $f, f^{\prime} \in Z^{n}(G, \alpha, K)$, dizemos que $f$ e $f^{\prime}$ são cohomologos se existe $g \in C^{n-1}(G, \alpha, K)$ tal que $f=f^{\prime}\left(\delta^{n} g\right)$. Neste caso, temos que $[f]=\left[f^{\prime}\right]$ em $H^{n}(G, \alpha, K)$.

Um $n$-cociclo $f$ é dito normalizado se

$$
f\left(1, x_{1}, \ldots, x_{n-1}\right)=f\left(x_{1}, 1, \ldots, x_{n-1}\right)=\ldots=f\left(x_{1}, \ldots, x_{n-1}, 1\right)=1_{x_{1}} 1_{x_{1} x_{2}} \ldots 1_{x_{1} \ldots x_{n-1}} .
$$

Observação 3.1.6. Todo 1-cociclo parcial $\sigma$ é normalizado. De fato, para todo $x, y \in G$ temos $\alpha_{x}\left(\sigma_{y} 1_{x-1}\right) \sigma_{x y}^{-1} \sigma_{x}=$ $1_{x} 1_{x y}$. Em particular, para $x=y=1$ temos $\sigma_{1}^{2}=\sigma_{1}$. Isso implica que $\sigma_{1}=1$. Portanto, $\sigma$ é normalizado.

Por [15, Observação 2.6] temos que se $\sigma \in Z^{2}(G, \alpha, \mathcal{Z})$, então existe $\widetilde{\sigma} \in Z^{2}(G, \alpha, \mathcal{Z})$ normalizado tal que $\sigma=\tilde{\sigma}\left(\delta^{1} \beta\right)$, para algum $\beta \in C^{1}(G, \alpha, \mathcal{Z})$. Logo, $\sigma$ é cohomologo à um 2-cociclo parcial normalizado. 


\subsection{Representação Parcial Unital}

Seja

$$
\begin{array}{rlr}
\Theta: \quad G & \longrightarrow \operatorname{PicS}(R) \\
x & \longmapsto & {\left[\Theta_{x}\right]}
\end{array}
$$

uma representação parcial. Dizemos que $\Theta$ é unital se $\varepsilon_{x}=\Theta_{x} \otimes_{R} \Theta_{x^{-1}} \simeq R 1_{x}$, onde $1_{x}$ é um idempotente central de $R$, para todo $x \in G$.

Lema 3.2.1. Seja $\Theta: G \longrightarrow \operatorname{PicS}(R)$ uma representação parcial unital com $\Theta_{x} \otimes_{R} \Theta_{x^{-1}} \simeq R 1_{x}$, para todo $x \in G$. Então, para todo $x, y \in G$, temos:

(i) $\Theta_{x} \otimes_{R} R 1_{y} \simeq R 1_{x y} \otimes_{R} \Theta_{x}$. Em particular,

$$
R 1_{x} \otimes_{R} \Theta_{x} \simeq \Theta_{x} \text { e } \Theta_{x} \otimes_{R} R 1_{x^{-1}} \simeq \Theta_{x} \text {, para todo } x \in G .
$$

(ii) $\Theta_{x} \otimes_{R} \Theta_{y} \simeq R 1_{x} \otimes_{R} \Theta_{x y}$ e $\Theta_{x} \otimes_{R} \Theta_{y} \simeq \Theta_{x y} \otimes_{R} R 1_{y^{-1}}$,

(iii) $\Theta_{x} \otimes_{R} \Theta_{y} \otimes_{R} \Theta_{(x y)^{-1}} \simeq R 1_{x} 1_{x y}$,

(iv) $\Theta_{x} \otimes_{R} \Theta_{y} \simeq \Theta_{x} \otimes_{R} \Theta_{y} \otimes_{R} R 1_{y^{-1} x^{-1}}$.

Demonstração. O item (i) segue diretamente de (3.2) e o item (ii) segue de (3.4) e (3.5). Para (iii) temos

$$
\begin{aligned}
\Theta_{x} \otimes_{R} \Theta_{y} \otimes_{R} \Theta_{(x y)^{-1}} & \stackrel{(i i)}{\simeq} R 1_{x} \otimes_{R} \Theta_{x y} \otimes_{R} \Theta_{(x y)^{-1}} \\
& \simeq R 1_{x} \otimes_{R} R 1_{x y} \simeq R 1_{x} 1_{x y} .
\end{aligned}
$$

Para (iv):

$$
\begin{aligned}
\Theta_{x} \otimes_{R} \Theta_{y} \otimes_{R} R 1_{y^{-1} x^{-1}} & \simeq \Theta_{x} \otimes_{R} \Theta_{y} \otimes_{R} \Theta_{(x y)^{-1}} \otimes_{R} \Theta_{x y} \\
& \simeq \Theta_{x} \otimes_{R} \Theta_{y} \otimes_{R} \Theta_{y^{-1}} \otimes_{R} \Theta_{x^{-1}} \otimes_{R} \Theta_{x y} \\
& \simeq \Theta_{x} \otimes_{R} \Theta_{y} \otimes_{R} \Theta_{y^{-1}} \otimes_{R} \Theta_{x^{-1}} \otimes_{R} \Theta_{x} \otimes_{R} \Theta_{y} \\
& \simeq \Theta_{x} \otimes_{R} R 1_{y} \otimes_{R} R 1_{x^{-1}} \otimes_{R} \Theta_{y} \\
& \simeq \Theta_{x} \otimes_{R} R 1_{x^{-1}} \otimes_{R} R 1_{y} \otimes_{R} \Theta_{y} \\
& \simeq \Theta_{x} \otimes_{R} \Theta_{y}
\end{aligned}
$$

Por (2.3) e pelos itens $(i)$ e (ii) do Lema 3.2.1, temos os isomorfismos de $R$-bimódulos

$$
1_{x y} \Theta_{x} \simeq \Theta_{x} 1_{y} \text { e } \Theta_{x} \otimes_{R} \Theta_{y} \simeq 1_{x} \Theta_{x y}, \text { para todo } x, y \in G .
$$

Observação 3.2.2. Dados $u_{x} \in \Theta_{x}, u_{y} \in \Theta_{y}$ e $u_{(x y)^{-1}} \in \Theta_{(x y)^{-1}}$, então

$$
r u_{x} \otimes u_{y} \otimes u_{(x y)^{-1}}=u_{x} \otimes u_{y} \otimes u_{(x y)^{-1}} r, \quad \text { para todo } r \in \mathcal{Z} .
$$

De fato, por (iii) do Lema 3.2.1 existe um isomorfismo de R-bimódulos $\varphi: \Theta_{x} \otimes_{R} \Theta_{y} \otimes_{R} \Theta_{(x y)^{-1}} \longrightarrow$ $R 1_{x} 1_{x y}$. Então, para todo $r \in \mathcal{Z}$ temos $r \varphi\left(u_{x} \otimes u_{y} \otimes u_{(x y)^{-1}}\right)=\varphi\left(u_{x} \otimes u_{y} \otimes u_{(x y)^{-1}}\right) r$. Por $\varphi$ ser $R$-bilinear segue que

$$
\varphi\left(r u_{x} \otimes u_{y} \otimes u_{(x y)^{-1}}\right)=\varphi\left(u_{x} \otimes u_{y} \otimes u_{(x y)^{-1}} r\right),
$$

e por $\varphi$ ser isomorfismo, temos portanto que $r u_{x} \otimes u_{y} \otimes u_{(x y)^{-1}}=u_{x} \otimes u_{y} \otimes u_{(x y)^{-1}} r$, para todo $r \in \mathcal{Z}$. 
Lema 3.2.3. Seja $\Theta: G \longrightarrow \operatorname{PicS}(R)$ uma representação parcial unital com $\varepsilon_{x}=\Theta_{x} \otimes_{R} \Theta_{x^{-1}} \simeq R 1_{x}$, onde $\Theta(x)=\left[\Theta_{x}\right]$ para todo $x \in G$. Então

$$
u_{x} 1_{y}=1_{x y} u_{x} 1_{y}=1_{x y} u_{x}, \quad \text { para todos } x, y \in G \text { e } u_{x} \in \Theta_{x} .
$$

Em particular, $1_{x y} \Theta_{x}=\Theta_{x} 1_{y}$, para todos $x, y \in G$.

Demonstração. Por (3.8) existe um isomorfismo de $R$-bimódulos $\kappa_{x, y}: \Theta_{x} 1_{y} \longrightarrow 1_{x y} \Theta_{x}$. Dado $u_{x} \in \Theta_{x}$, existe $v_{x} \in \Theta_{x}$ tal que $\kappa_{x, y}\left(u_{x} 1_{y}\right)=1_{x y} v_{x}$. Multiplicando à esquerda por $1_{x y}$ temos

$$
\kappa_{x, y}\left(1_{x y} u_{x} 1_{y}\right)=1_{x y} v_{x}=\kappa_{x, y}\left(u_{x} 1_{y}\right) .
$$

Como $\kappa_{x, y}$ é isomorfismo, temos que $1_{x y} u_{x} 1_{y}=u_{x} 1_{y}$. Por outro lado, existe $v_{x}^{\prime} \in \Theta_{x}$ tal que $\kappa_{x, y}^{-1}\left(1_{x y} u_{x}\right)=$ $v_{x}^{\prime} 1_{y}$. Multiplicando à direita por $1_{y}$ temos

$$
\kappa_{x, y}^{-1}\left(1_{x y} u_{x} 1_{y}\right)=v_{x}^{\prime} 1_{y}=\kappa_{x, y}^{-1}\left(1_{x y} u_{x}\right) .
$$

O que implica $1_{x y} u_{x} 1_{y}=1_{x y} u_{x}$. Temos portanto que

$$
u_{x} 1_{y}=1_{x y} u_{x} 1_{y}=1_{x y} u_{x}, \text { para todo } u_{x} \in \Theta_{x} .
$$

Segue, em particular, do Lema 3.2.3 que

$$
1_{x} u_{x}=u_{x} \text { e } u_{x} 1_{x^{-1}}=u_{x} \text {, para todo } x \in G, u_{x} \in \Theta_{x} .
$$

Então, as aplicações $R 1_{x} \otimes_{R} \Theta_{x} \simeq \Theta_{x}$ e $\Theta_{x} \otimes_{R} R 1_{x^{-1}} \simeq \Theta_{x}$ definidos pela ação à esquerda e ação à direita, respectivamente, são isomorfismos de $R$-bimódulos. Logo, $\Theta_{x}$ é um $R 1_{x^{-}} R 1_{x^{-1}}$-bimódulo unital e existem isomorfismos de $R$-bimódulos

$$
\tau_{x}: \Theta_{x} \otimes_{R} \Theta_{x^{-1}} \longrightarrow R 1_{x} \text { e } \tau_{x^{-1}}: \Theta_{x^{-1}} \otimes_{R} \Theta_{x} \longrightarrow R 1_{x^{-1}} .
$$

Note que os $R 1_{x^{-}} R 1_{x^{-1}}$-bimódulos $\Theta_{x} \otimes_{R} \Theta_{x^{-1}}$ e $\Theta_{x} \otimes_{R 1_{x^{-1}}} \Theta_{x^{-1}}$ coincidem, pois por (3.11) temos

$$
u_{x} r \otimes_{R 1_{x^{-1}}} u_{x^{-1}}=u_{x} 1_{x^{-1}} r \otimes_{R 1_{x^{-1}}} u_{x^{-1}}=u_{x} \otimes_{R 1_{x^{-1}}} r 1_{x^{-1}} u_{x^{-1}}=u_{x} \otimes_{R 1_{x^{-1}}} r u_{x^{-1}},
$$

para todo $u_{x} \in \Theta_{x}, u_{x^{-1}} \in \Theta_{x^{-1}}$ e $r \in R$. Pela Proposição 2.1.11 podemos escolher os isomorfismos $\tau_{x}$ e $\tau_{x^{-1}}$ de tal forma que valem as igualdades em (2.6). Para simplificar a notação, vamos denotar $\tau_{x}\left(u_{x} \otimes u_{x^{-1}}\right)=u_{x} u_{x^{-1}}$, para todo $u_{x} \in \Theta_{x}$ e $u_{x^{-1}} \in \Theta_{x^{-1}}$. Então, por (2.6) temos

$$
\left(u_{x} u_{x^{-1}}\right) u_{x}^{\prime}=u_{x}\left(u_{x^{-1}} u_{x}^{\prime}\right) \text { e }\left(u_{x^{-1}} u_{x}\right) u_{x^{-1}}^{\prime}=u_{x^{-1}}\left(u_{x} u_{x^{-1}}^{\prime}\right),
$$

para todo $u_{x}, u_{x}^{\prime} \in \Theta_{x}$ e $u_{x^{-1}}, u_{x^{-1}}^{\prime} \in \Theta_{x^{-1}}$.

\subsubsection{Ação parcial sobre $\mathcal{Z}$ e sobre $\operatorname{PicS}_{\mathcal{Z}}(R)$.}

Seja

$$
\begin{aligned}
\Theta: \quad G & \longrightarrow \operatorname{PicS}(R) \\
x & \longmapsto\left[\Theta_{x}\right]
\end{aligned}
$$

uma representação parcial unital com $\Theta_{x} \otimes_{R} \Theta_{x^{-1}} \simeq R 1_{x}$, para todo $x \in G$. 
Teorema 3.2.4. Considere $D_{x}=\mathcal{Z} 1_{x}$ é o ideal de $\mathcal{Z}$ gerado por $1_{x}$ e

$$
\begin{aligned}
\alpha_{x}: D_{x^{-1}} & \longrightarrow \sum_{(x)} \omega_{x} r \omega_{x^{-1}}, \\
r & \longmapsto \sum
\end{aligned}
$$

onde $\omega_{x} \in \Theta_{x}$ e $\omega_{x^{-1}} \in \Theta_{x^{-1}}$ são tais que $1_{x}=\sum_{(x)} \omega_{x} \omega_{x^{-1}}$. Então, $\alpha=\left(D_{x}, \alpha_{x}\right)_{x \in G}$ é uma ação parcial de $G$ sobre $\mathcal{Z}$.

Demonstração. Primeiramente, vejamos que $\alpha_{x}$ não depende da escolha dos representantes da decomposição de $1_{x}$. Sejam $\widetilde{\omega}_{x} \in \Theta_{x}$ e $\widetilde{\omega}_{x^{-1}} \in \Theta_{x^{-1}}$ tais que $\sum_{(\widetilde{x})} \widetilde{\omega}_{x} \widetilde{\omega}_{x^{-1}}=1_{x}$. Então,

$$
\begin{aligned}
& \alpha_{x}\left(r 1_{x^{-1}}\right)=\sum_{(x)} \omega_{x} r \omega_{x^{-1}} \stackrel{(3.11)}{=} \sum_{(x)} \omega_{x} r \omega_{x^{-1}} 1_{x}=\sum_{(x),(\widetilde{x})} \omega_{x} r \omega_{x^{-1}}\left(\widetilde{\omega}_{x} \widetilde{\omega}_{x^{-1}}\right) \\
& \stackrel{(3.12)}{=} \sum_{(x),(\widetilde{x})} \omega_{x} r\left(\omega_{x^{-1}} \widetilde{\omega}_{x}\right) \widetilde{\omega}_{x^{-1}} \stackrel{r \in \mathcal{Z}}{=} \sum_{(x),(\widetilde{x})} \omega_{x}\left(\omega_{x^{-1}} \widetilde{\omega}_{x}\right) r \widetilde{\omega}_{x^{-1}} \\
& \stackrel{(3.12)}{=} \sum_{(x),(\widetilde{x})}\left(\omega_{x} \omega_{x^{-1}}\right) \widetilde{\omega}_{x} r \widetilde{\omega}_{x^{-1}}=\sum_{(\widetilde{x})} 1_{x} \widetilde{\omega}_{x} r \widetilde{\omega}_{x^{-1}} \stackrel{(3.11)}{=} \sum_{(\widetilde{x})} \widetilde{\omega}_{x} r \widetilde{\omega}_{x^{-1}} .
\end{aligned}
$$

Dado $r^{\prime} \in R$ temos

$$
\begin{aligned}
\alpha_{x}\left(r 1_{x^{-1}}\right) r^{\prime} & =\sum_{(x)}\left(\omega_{x} r \omega_{x^{-1}}\right) r^{\prime}=\sum_{(x)} \omega_{x} r \omega_{x^{-1}} 1_{x} r^{\prime}=\sum_{(x),(\widetilde{x})} \omega_{x} r \omega_{x^{-1}} r^{\prime}\left(\widetilde{\omega}_{x} \widetilde{\omega}_{x^{-1}}\right) \\
& =\sum_{(x),(\widetilde{x})} \omega_{x} r\left(\omega_{x^{-1}} r^{\prime} \widetilde{\omega}_{x}\right) \widetilde{\omega}_{x^{-1}} r \in \mathcal{Z} \\
& =\sum_{(x),(\widetilde{x})} \omega_{x}\left(\omega_{x-1} r^{\prime} \widetilde{\omega}_{x}\right) r \widetilde{\omega}_{x^{-1}} \\
& =\sum_{(x),(\widetilde{x})} r^{\prime} \widetilde{\omega}_{x} r \widetilde{\omega}_{x^{-1}} r^{\prime} \widetilde{\omega}_{x} r \widetilde{\omega}_{x^{-1}}=\sum_{(\widetilde{x})} 1_{x} r^{\prime} \widetilde{\omega}_{x} r \widetilde{\omega}_{x^{-1}}
\end{aligned}
$$

Logo, $\alpha_{x}\left(r 1_{x^{-1}}\right) \in D_{x}=\mathcal{Z} 1_{x}$.

Vejamos agora $\alpha_{x}$ é um isomorfismo com inverso $\alpha_{x^{-1}}$. Sejam $\bar{\omega}_{x} \in \Theta_{x}$ e $\bar{\omega}_{x^{-1}} \in \Theta_{x^{-1}}$, tais que $\sum_{(\bar{x})} \bar{\omega}_{x^{-1}} \bar{\omega}_{x}=1_{x^{-1}}$. Então, dado $r \in D_{x}$, temos

$$
\begin{aligned}
\left(\alpha_{x} \circ \alpha_{x^{-1}}\right)(r) & =\alpha_{x}\left(\sum_{(\bar{x})} \bar{\omega}_{x^{-1}} r \bar{\omega}_{x}\right)=\sum_{(x),(\bar{x})} \omega_{x}\left(\bar{\omega}_{x^{-1}} r \bar{\omega}_{x}\right) \omega_{x^{-1}} \\
& =\sum_{(x),(\bar{x})}\left(\omega_{x} \bar{\omega}_{x^{-1}}\right) r \bar{\omega}_{x} \omega_{x^{-1}} \stackrel{r \in Z}{=} \sum_{(x),(\bar{x})} r\left(\omega_{x} \bar{\omega}_{x^{-1}}\right) \bar{\omega}_{x} \omega_{x^{-1}} \\
& =\sum_{(x),(\bar{x})} r \omega_{x}\left(\bar{\omega}_{x^{-1}} \bar{\omega}_{x}\right) \omega_{x^{-1}}=\sum_{(x)} r \omega_{x} 1_{x^{-1}} \omega_{x^{-1}} \\
& =\sum_{(x)} r \omega_{x} \omega_{x^{-1}}=r 1_{x}=r .
\end{aligned}
$$


Analogamente, dado $r \in D_{x^{-1}}$, temos que $\left(\alpha_{x^{-1}} \circ \alpha_{x}\right)(r)=r$. Portanto, $\alpha_{x}^{-1}=\alpha_{x^{-1}}$. Dados $r, s \in D_{x}$ temos

$$
\begin{aligned}
\alpha_{x}(r) \alpha_{x}(s) & =\sum_{(x),(\widetilde{x})}\left(\omega_{x} r \omega_{x^{-1}}\right)\left(\widetilde{\omega}_{x} s \widetilde{\omega}_{x^{-1}}\right) \stackrel{(3.12)}{=} \sum_{(x),(\widetilde{x})} \omega_{x} r\left(\omega_{x^{-1}} \widetilde{\omega}_{x}\right) s \widetilde{\omega}_{x^{-1}} \\
& \stackrel{s \in \mathcal{Z}}{=} \sum_{(x),(\widetilde{x})} \omega_{x} r s\left(\omega_{x^{-1}} \widetilde{\omega}_{x}\right) \widetilde{\omega}_{x^{-1}} \stackrel{(3.12)}{=} \sum_{(x),(\widetilde{x})} \omega_{x} r s \omega_{x^{-1}}\left(\widetilde{\omega}_{x} \widetilde{\omega}_{x^{-1}}\right) \\
& =\sum_{(x)} \omega_{x} r s \omega_{x^{-1}} 1_{x} \stackrel{(3.11)}{=} \sum_{(x)} \omega_{x} r s \omega_{x^{-1}}=\alpha_{x}(r s) .
\end{aligned}
$$

Logo, $\alpha_{x}$ é um isomorfismo de anéis.

Dado $r \in D_{y} \cap D_{x^{-1}}$ e $1_{y^{-1}}=\sum_{(\bar{y})} \bar{\omega}_{y^{-1}} \bar{\omega}_{y}$, temos

$$
\alpha_{y^{-1}}(r)=\sum_{(\bar{y})} \bar{\omega}_{y^{-1}} r \bar{\omega}_{y}=\sum_{(\bar{y})} \bar{\omega}_{y^{-1}} r 1_{x^{-1}} \bar{\omega}_{y}=\sum_{(\bar{y})} \bar{\omega}_{y^{-1}} r \bar{\omega}_{y} 1_{(x y)^{-1}} \in D_{(x y)^{-1}} .
$$

Logo, temos $\alpha_{y^{-1}}\left(D_{y} \cap D_{x^{-1}}\right) \subseteq D_{(x y)^{-1}}$. Observe que

$$
1_{x} 1_{x y}=\sum_{(x)} \omega_{x} \omega_{x^{-1}} 1_{x y}=\sum_{(x)} \omega_{x} 1_{y} \omega_{x^{-1}}=\sum_{(x),(y)} \omega_{x}\left(\omega_{y} \omega_{y^{-1}}\right) \omega_{x-1}
$$

Então, para $r \in D_{y^{-1}} \cap D_{(x y)^{-1}}$ temos

$$
\begin{aligned}
\alpha_{x y}(r) & =\sum_{(x y)} \omega_{x y} r \omega_{(x y)^{-1}}, \text { onde } \sum_{(x y)} \omega_{x y} \omega_{(x y)^{-1}}=1_{x y} \\
\stackrel{r \in D_{y}^{-1}}{=} & \sum_{(x y)} 1_{x y} \omega_{x y} 1_{y^{-1}} r \omega_{(x y)^{-1}} \stackrel{(3.10)}{=} \sum_{(x y)} 1_{x y} 1_{x} \omega_{x y} r \omega_{(x y)^{-1}} \\
\stackrel{(3.13)}{=} & \sum_{\substack{(x),(y),(x y)}}\left(\omega_{x}\left(\omega_{y} \omega_{y^{-1}}\right) \omega_{x^{-1}}\right) \omega_{x y} r \omega_{(x y)^{-1}}
\end{aligned}
$$

Porém,

$$
\begin{aligned}
\left(\omega_{x}\left(\omega_{y} \omega_{y^{-1}}\right) \omega_{x^{-1}}\right) \omega_{x y} r \omega_{(x y)^{-1}}= & \tau_{x y} \circ\left(\tau_{x} \otimes \Theta_{x y} \otimes \Theta_{(x y)^{-1}}\right) \circ\left(\Theta_{x} \otimes \tau_{y} \otimes \Theta_{x^{-1}} \otimes \Theta_{x y} \otimes \Theta_{(x y)^{-1}}\right) \\
& \left(\omega_{x} \otimes \omega_{y} \otimes \omega_{y^{-1}} \otimes \omega_{x^{-1}} \otimes \omega_{x y} r \otimes \omega_{(x y)^{-1}}\right) \\
\stackrel{(3.9)}{=} & \tau_{x y} \circ\left(\tau_{x} \otimes \Theta_{x y} \otimes \Theta_{(x y)^{-1}}\right) \circ\left(\Theta_{x} \otimes \tau_{y} \otimes \Theta_{x^{-1}} \otimes \Theta_{x y} \otimes \Theta_{(x y)^{-1}}\right) \\
& \left(\omega_{x} \otimes \omega_{y} \otimes r \omega_{y^{-1}} \otimes \omega_{x^{-1}} \otimes \omega_{x y} \otimes \omega_{(x y)^{-1}}\right) \\
= & \left(\omega_{x}\left(\omega_{y} r \omega_{y^{-1}}\right) \omega_{x^{-1}}\right) \omega_{x y} \omega_{(x y)^{-1}}
\end{aligned}
$$

Assim,

$$
\begin{aligned}
\alpha_{x y}(r) & =\sum_{\substack{(x),(y) \\
(x y)}}\left(\omega_{x}\left(\omega_{y} r \omega_{y^{-1}}\right) \omega_{x^{-1}}\right) \omega_{x y} \omega_{(x y)^{-1}} \\
& =\sum_{(x),(y)}\left(\omega_{x}\left(\omega_{y} r \omega_{y^{-1}}\right) \omega_{x^{-1}}\right) 1_{x y} \\
\stackrel{(3.10)}{=} \sum_{(x),(y)}\left(\omega_{x}\left(\omega_{y} r \omega_{y^{-1}}\right) 1_{y} \omega_{x^{-1}}\right) & \\
& =\sum_{(x),(y)}\left(\omega_{x}\left(\omega_{y} r \omega_{y^{-1}}\right) \omega_{x^{-1}}\right) \\
& =\alpha_{x} \circ \alpha_{y}(r) .
\end{aligned}
$$


Portanto, $\alpha=\left(D_{x}, \alpha_{x}\right)_{x \in G}$ é uma ação parcial de $G$ sobre $\mathcal{Z}$.

Lema 3.2.5. Seja $\alpha$ a ação parcial sobre $\mathcal{Z}$ construída no Teorema 3.2.4. Então:

(i) Para todo $u_{x} \in \Theta_{x}$ e $r \in \mathcal{Z}$ temos

$$
\alpha_{x}\left(r 1_{x^{-1}}\right) u_{x}=u_{x} r .
$$

(ii) Se $M \mid \Theta_{x}$, então para todo $m \in M$ e $r \in \mathcal{Z}$ temos

$$
\alpha_{x}\left(r 1_{x^{-1}}\right) m=m r .
$$

Demonstração. (i) Dado $r \in \mathcal{Z}$ e $u_{x} \in \Theta_{x}$ temos que

$$
\begin{aligned}
\alpha_{x}\left(r 1_{x^{-1}}\right) u_{x} & =\sum_{(x)}\left(\omega_{x} r \omega_{x^{-1}}\right) u_{x} \stackrel{(3.12)}{=} \sum_{(x)} \omega_{x} r\left(\omega_{x^{-1}} u_{x}\right) \\
r \stackrel{\in \mathcal{Z}}{=} & \sum_{(x)} \omega_{x}\left(\omega_{x^{-1}} u_{x}\right) r \stackrel{(3.12)}{=} \sum_{(x)}\left(\omega_{x} \omega_{x^{-1}}\right) u_{x} r \\
& =1_{x} u_{x} r \stackrel{(3.11)}{=} u_{x} r .
\end{aligned}
$$

(ii) Sejam $f_{i}: M \longrightarrow \Theta_{x}$ e $g_{i}: \Theta_{x} \longrightarrow M$, com $i=1,2, \ldots, n$ morfismos de $R$-bimódulos tais que $\sum_{i=1}^{n} g_{i} f_{i}=I d_{M}$. Dados $m \in M$ e $r \in \mathcal{Z}$ temos

$$
\begin{aligned}
m r & =\sum_{i=1}^{n} g_{i}\left(f_{i}(m)\right) r=\sum_{i=1}^{n} g_{i}(\underbrace{f_{i}(m)}_{\in \Theta_{x}} r) \\
\stackrel{(3.14)}{=} & \sum_{i=1}^{n} g_{i}\left(\alpha_{x}\left(r 1_{x^{-1}}\right) f_{i}(m)\right) \\
& =\sum_{i=1}^{n} \alpha_{x}\left(r 1_{x^{-1}}\right) g_{i}\left(f_{i}(m)\right) \\
& =\alpha_{x}\left(r 1_{x^{-1}}\right) m .
\end{aligned}
$$

Observação 3.2.6. Para cada representação parcial unital $\Theta: G \longrightarrow \operatorname{PicS}(R)$ construímos uma ação parcial $\alpha$ de $G$ sobre $\mathcal{Z}$ como em (3.2.4). Consequentemente, temos os grupos de cohomologias parciais $H_{\Theta}^{*}(G, \alpha, \mathcal{Z})$ associados a essa ação. Seguindo o caso global, nas próximas seções vamos fixar uma representação parcial unital $\Theta$ e consequentemente a ação parcial sobre $\mathcal{Z}$.

Lema 3.2.7. Seja $\Theta: G \longrightarrow \operatorname{PicS}(R)$ uma representação parcial unital com $\varepsilon_{x}=\Theta_{x} \otimes_{R} \Theta_{x^{-1}} \simeq R 1_{x}$, onde $\Theta(x)=\left[\Theta_{x}\right]$, para todo $x \in G$. Existe um isomorfismo de grupos

$$
\begin{array}{cl}
\operatorname{Aut}_{R-R}\left(1_{y} \Theta_{x}\right) & \stackrel{\widetilde{(-)}}{\longmapsto} \mathcal{U}\left(\mathcal{Z} 1_{x} 1_{y}\right) \\
\sigma & \longmapsto \widetilde{\sigma}
\end{array}
$$

onde

$$
\widetilde{\sigma}=\sum_{(x)} \sigma\left(1_{y} \omega_{x}\right) \omega_{x^{-1}},
$$


$\operatorname{com} \sum_{(x)} \omega_{x} \omega_{x^{-1}}=1_{x}$. Além disso, temos

$$
\sigma\left(1_{y} u_{x}\right)=\widetilde{\sigma} u_{x}, \text { para todo } u_{x} \in \Theta_{x}
$$

Demonstração. Vejamos primeiro que $\widetilde{\sigma}$ não depende da decomposição de $1_{x}$. Sejam

$$
1_{x}=\sum_{(x)} \omega_{x} \omega_{x^{-1}}=\sum_{(\widetilde{x})} \widetilde{\omega}_{x} \widetilde{\omega}_{x^{-1}}
$$

$\operatorname{com} \omega_{x}, \widetilde{\omega}_{x} \in \Theta_{x}$ e $\omega_{x^{-1}}, \widetilde{\omega}_{x^{-1}} \in \Theta_{x^{-1}}$. Então,

$$
\begin{aligned}
\widetilde{\sigma} & =\sum_{(x)} \sigma\left(1_{y} \omega_{x}\right) \omega_{x^{-1}}=\sum_{(x)} \sigma\left(1_{y} \omega_{x}\right) \omega_{x^{-1}} 1_{x}=\sum_{(x),(\widetilde{x})} \sigma\left(1_{y} \omega_{x}\right) \omega_{x^{-1}}\left(\widetilde{\omega}_{x} \widetilde{\omega}_{x^{-1}}\right) \\
& =\sum_{(x),(\widetilde{x})} \sigma\left(1_{y} \omega_{x}\right)\left(\omega_{x^{-1}} \widetilde{\omega}_{x}\right) \widetilde{\omega}_{x^{-1}}=\sum_{(x),(\widetilde{x})} \sigma\left(1_{y} \omega_{x}\left(\omega_{x^{-1}} \widetilde{\omega}_{x}\right)\right) \widetilde{\omega}_{x^{-1}} \\
& =\sum_{(x),(\widetilde{x})} \sigma\left(1_{y}\left(\omega_{x} \omega_{x^{-1}}\right) \widetilde{\omega}_{x}\right) \widetilde{\omega}_{x^{-1}}=\sum_{(\widetilde{x})} \sigma\left(1_{y} 1_{x} \widetilde{\omega}_{x}\right) \widetilde{\omega}_{x^{-1}} \\
& =\sum_{(\widetilde{x})} \sigma\left(1_{y} \widetilde{\omega}_{x}\right) \widetilde{\omega}_{x^{-1}} .
\end{aligned}
$$

Claramente $\widetilde{\sigma} \in R 1_{x} 1_{y}$. Dado $r \in R$ temos:

$$
\begin{aligned}
\widetilde{\sigma} r & =\sum_{(x)} \sigma\left(1_{y} \omega_{x}\right) \omega_{x^{-1}} r=\sum_{(x)} \sigma\left(1_{y} \omega_{x}\right) \omega_{x^{-1}} r 1_{x}=\sum_{(x),(\widetilde{x})} \sigma\left(1_{y} \omega_{x}\right) \omega_{x^{-1}} r\left(\widetilde{\omega}_{x} \widetilde{\omega}_{x^{-1}}\right) \\
& =\sum_{(x),(\widetilde{x})} \sigma\left(1_{y} \omega_{x}\right)\left(\omega_{x^{-1}} r \widetilde{\omega}_{x}\right) \widetilde{\omega}_{x^{-1}}=\sum_{(x),(\widetilde{x})} \sigma\left(1_{y} \omega_{x}\left(\omega_{x^{-1}} r \widetilde{\omega}_{x}\right)\right) \widetilde{\omega}_{x^{-1}} \\
& =\sum_{(x),(\widetilde{x})} \sigma\left(1_{y}\left(\omega_{x} \omega_{x^{-1}}\right) r \widetilde{\omega}_{x}\right) \widetilde{\omega}_{x^{-1}}=\sum_{(\widetilde{x})} \sigma\left(1_{y} 1_{x} r \widetilde{\omega}_{x}\right) \widetilde{\omega}_{x^{-1}} \\
& =\sum_{(\widetilde{x})} r \sigma\left(1_{y} \widetilde{\omega}_{x}\right) \widetilde{\omega}_{x^{-1}}=r \widetilde{\sigma} .
\end{aligned}
$$

Logo, $\widetilde{\sigma} \in \mathcal{Z} 1_{x} 1_{y}$. Resta ver que $\widetilde{\sigma}$ é inversível em $\mathcal{Z} 1_{x} 1_{y}$. Como $\sigma$ é isomorfismo, considere $\widetilde{\sigma^{-1}}$. Temos

$$
\begin{aligned}
\widetilde{\sigma} \sigma^{-1} & =\sum_{(x),(\widetilde{x})}\left(\sigma\left(1_{y} \omega_{x}\right) \omega_{x^{-1}}\right)\left(\sigma^{-1}\left(1_{y} \widetilde{\omega}_{x}\right) \widetilde{\omega}_{x^{-1}}\right) \\
& =\sum_{(x),(\widetilde{x})} \sigma\left(1_{y} \omega_{x}\right)\left(\omega_{x^{-1}} \sigma^{-1}\left(1_{y} \widetilde{\omega}_{x}\right)\right) \widetilde{\omega}_{x^{-1}} \\
& =\sum_{(x),(\widetilde{x})} \sigma\left(1_{y} \omega_{x} \omega_{x^{-1}} \sigma^{-1}\left(1_{y} \widetilde{\omega}_{x}\right)\right) \widetilde{\omega}_{x^{-1}} \\
& =\sum_{(\widetilde{x})} \sigma\left(1_{y} 1_{x} \sigma^{-1}\left(1_{y} \widetilde{\omega}_{x}\right)\right) \widetilde{\omega}_{x^{-1}} \\
& =\sum_{(\widetilde{x})} \sigma\left(\sigma^{-1}\left(1_{y} \widetilde{\omega}_{x}\right)\right) \widetilde{\omega}_{x^{-1}} \\
& =\sum_{(\widetilde{x})} 1_{y} \widetilde{\omega}_{x} \widetilde{\omega}_{x^{-1}}=1_{y} 1_{x} .
\end{aligned}
$$

Analogamente, temos que $\widetilde{\sigma^{-1}} \widetilde{\sigma}=1_{x} 1_{y}$. Logo, $\widetilde{\sigma} \in \mathcal{U}\left(\mathcal{Z} 1_{x} 1_{y}\right)$. Além disso, dado $u_{x} \in \Theta_{x}$, temos:

$$
\widetilde{\sigma} u_{x}=\sum_{(x)} \sigma\left(1_{y} \omega_{x}\right) \omega_{x^{-1}} u_{x}=\sum_{(x)} \sigma\left(1_{y} \omega_{x} \omega_{x-1} u_{x}\right)=\sigma\left(1_{y} 1_{x} u_{x}\right)=\sigma\left(1_{y} u_{x}\right) .
$$


Portanto, vale (3.17).

Considere agora $r \in \mathcal{U}\left(\mathcal{Z} 1_{x} 1_{y}\right)$. Defina

$$
\begin{aligned}
\sigma_{r}: 1_{y} \Theta_{x} & \longrightarrow 1_{y} \Theta_{x} \\
v_{x} & \longmapsto r v_{x} .
\end{aligned}
$$

Claramente $\sigma_{r}$ é $R$-linear à direita. Como $r \in \mathcal{Z}$ temos que $\sigma_{r}$ é $R$-linear à esquerda. De fato, dado $r^{\prime} \in R$ temos

$$
\sigma_{r}\left(r^{\prime} v_{x}\right)=r r^{\prime} v_{x}=r^{\prime} r v_{x}=r^{\prime} \sigma_{r}\left(v_{x}\right) \text {. }
$$

Seja $r^{-1}$ o inverso de $r$ em $\mathcal{Z} 1_{x} 1_{y}$, então $r r^{-1}=r^{-1} r=1_{x} 1_{y}$. Então, a inversa de $\sigma_{r}$ é dada por

$$
\begin{aligned}
\sigma_{r}^{-1}: 1_{y} \Theta_{x} & \longrightarrow 1_{y} \Theta_{x} \\
v_{x} & \longmapsto r^{-1} v_{x} .
\end{aligned}
$$

De fato, dado $v_{x} \in 1_{y} \Theta_{x}$, temos

$$
\sigma_{r}^{-1}\left(\sigma_{r}\left(v_{x}\right)\right)=\sigma_{r}^{-1}\left(r v_{x}\right)=r^{-1}\left(r v_{x}\right)=\left(r^{-1} r\right) v_{x}=1_{x} 1_{y} v_{x}=v_{x} .
$$

Analogamente, $\sigma_{r}\left(\sigma_{r}^{-1}\left(v_{x}\right)\right)=v_{x}$, para todo $v_{x} \in 1_{y} \Theta_{x}$. Logo, $\sigma_{r} \in \mathbf{A u t}_{R-R}\left(1_{y} \Theta_{x}\right)$. Observe agora que

$$
\widetilde{\sigma_{r}}=\sum_{(x)} \sigma_{r}\left(1_{y} \omega_{x}\right) \omega_{x^{-1}}=\sum_{(x)} r 1_{y} \omega_{x} \omega_{x^{-1}}=r 1_{y} 1_{x}=r .
$$

Por outro lado, dado $\sigma \in \mathbf{A u t}_{R-R}\left(1_{y} \Theta_{x}\right)$ considere

$$
\begin{aligned}
\gamma: 1_{y} \Theta_{x} & \longrightarrow 1_{y} \Theta_{x} \\
v_{x} & \longmapsto \widetilde{\sigma} v_{x} .
\end{aligned}
$$

Por (3.17) temos:

$$
\gamma\left(v_{x}\right)=\widetilde{\sigma} v_{x}=\sigma\left(1_{y} v_{x}\right)=\sigma\left(v_{x}\right), \text { para todo } v_{x} \in 1_{y} \Theta_{x} .
$$

Logo, $\gamma=\sigma$ e portanto $\widetilde{(-)}$ é um isomorfismo.

Resta verificar que $\widetilde{(-)}$ é um morfismo de grupos. Sejam $\sigma, \gamma \in \mathbf{A u t}_{R-R}\left(1_{y} \Theta_{x}\right)$, então

$$
\begin{aligned}
\widetilde{\sigma} \widetilde{\gamma} & =\sum_{(x),(\widetilde{x})}\left(\sigma\left(1_{y} \omega_{x}\right) \omega_{x^{-1}}\right)\left(\gamma\left(1_{y} \widetilde{\omega}_{x}\right) \widetilde{\omega}_{x^{-1}}\right)=\sum_{(x),(\widetilde{x})} \sigma\left(1_{y} \omega_{x}\right)\left(\omega_{x^{-1}} \gamma\left(1_{y} \widetilde{\omega}_{x}\right)\right) \widetilde{\omega}_{x^{-1}} \\
& =\sum_{(x),(\widetilde{x})} \sigma\left(1_{y} \omega_{x} \omega_{x^{-1}} \gamma\left(1_{y} \widetilde{\omega}_{x}\right)\right) \widetilde{\omega}_{x^{-1}}=\sum_{(\widetilde{x})} \sigma\left(1_{y} 1_{x} \gamma\left(1_{y} \widetilde{\omega}_{x}\right)\right) \widetilde{\omega}_{x^{-1}} \\
& =\sum_{(\widetilde{x})} \sigma\left(\gamma\left(1_{y} \widetilde{\omega}_{x}\right)\right) \widetilde{\omega}_{x^{-1}}=\widetilde{\sigma \circ \gamma} .
\end{aligned}
$$

Vamos agora construir uma ação parcial sobre $\mathbf{P i c S}(R)$ : Seja $\mathcal{X}_{x}=\left[R 1_{x}\right] \mathbf{P i c S}(R)\left[R 1_{x}\right]$ e

$$
\begin{aligned}
\alpha_{x}^{*}: \mathcal{X}_{x-1} & \longrightarrow \mathcal{X}_{x} \\
{[P] } & \longmapsto\left[\Theta_{x} \otimes_{R} P \otimes_{R} \Theta_{x^{-1}}\right]
\end{aligned}
$$

Pela Proposição 3.1.3, temos que $\alpha^{*}=\left(\mathcal{X}_{x}, \alpha_{x}^{*}\right)$ é uma ação parcial sobre $\mathbf{P i c} \mathbf{S}(R)$. Observe que se $[P] \in \operatorname{PicS}_{\mathcal{Z}}(R)$, então pelo Corolário 2.3.6, temos que $P \otimes_{R} R 1_{x} \simeq R 1_{x} \otimes_{R} P$, como $R$-bimódulos, para todo $x \in G$. Logo, $\left[R 1_{x}\right]$ é um idempotente central em $\mathbf{P i c S}_{\mathcal{Z}}(R)$. Além disso, se $[P] \in \mathbf{P i c S}_{\mathcal{Z}}(R)$, então $\left[\Theta_{x} \otimes_{R} P \otimes_{R} \Theta_{x^{-1}}\right] \in \mathbf{P i c S}_{\mathcal{Z}}(R)$. De fato, basta verificar que $\Theta_{x} \otimes_{R} P \otimes_{R} \Theta_{x^{-1}}$ também é $\mathcal{Z}$-bimódulo 
central. Sejam $p \in P, u_{x} \in \Theta_{x}, u_{x^{-1}} \in \Theta_{x^{-1}}$ e $r \in \mathcal{Z}$, então por $P$ ser $\mathcal{Z}$-bimódulo central e por (3.14) temos

$$
\begin{aligned}
u_{x} \otimes p \otimes u_{x^{-1}} r & =u_{x} \otimes p \otimes \alpha_{x^{-1}}\left(r 1_{x}\right) u_{x^{-1}}=u_{x} \otimes p \alpha_{x^{-1}}\left(r 1_{x}\right) \otimes u_{x^{-1}} \\
& =u_{x} \otimes \alpha_{x^{-1}}\left(r 1_{x}\right) p \otimes u_{x^{-1}}=u_{x} \alpha_{x^{-1}}\left(r 1_{x}\right) \otimes p \otimes u_{x^{-1}} \\
& =\alpha_{x}\left(\alpha_{x^{-1}}\left(r 1_{x}\right)\right) u_{x} \otimes p \otimes u_{x^{-1}}=r 1_{x} u_{x} \otimes p \otimes u_{x^{-1}} \\
& =r u_{x} \otimes p \otimes u_{x^{-1}} .
\end{aligned}
$$

Assim, restringindo a ação parcial acima a $\operatorname{PicS}_{\mathcal{Z}}(R)$, temos que $\mathcal{X}_{x}=\operatorname{PicS}_{\mathcal{Z}}(R)\left[R 1_{x}\right]$ é o ideal de $\operatorname{PicS}_{\mathcal{Z}}(R)$ gerado pelo idempotente central $\left[R 1_{x}\right]$ e

$$
\begin{aligned}
\alpha_{x}^{*}: \quad \operatorname{PicS}_{\mathcal{Z}}(R)\left[R 1_{x^{-1}}\right] & \longrightarrow \operatorname{PicS}_{\mathcal{Z}}(R)\left[R 1_{x}\right] \\
{[P]\left[R 1_{x^{-1}}\right] } & \longmapsto\left[\Theta_{x} \otimes_{R} P \otimes_{R} \Theta_{x^{-1}}\right]
\end{aligned}
$$

está bem definido. Portanto, $\alpha^{*}=\left(\mathcal{X}_{x}, \alpha_{x}^{*}\right)$ é uma ação parcial sobre $\mathbf{P i c} \mathbf{S}_{\mathcal{Z}}(R)$.

O conjunto dos invariantes $\operatorname{de} \mathbf{P i c S}_{\mathcal{Z}}(R)$ é dado por

$$
\operatorname{PicS}_{\mathcal{Z}}(R)^{\alpha^{*}}=\left\{[P] \in \operatorname{PicS}_{\mathcal{Z}}(R) ; \Theta_{x} \otimes_{R} P \simeq P \otimes_{R} \Theta_{x} \text {, para todo } x \in G\right\} .
$$

De fato, $[P] \in \operatorname{PicS}_{\mathcal{Z}}(R)^{\alpha^{*}}$ se, e somente se, $\alpha_{x}^{*}\left([P]\left[R 1_{x^{-1}}\right]\right)=[P]\left[R 1_{x}\right]$, para todo $x \in G$. Segue que:

$$
\begin{aligned}
{[P] \in \mathbf{P i c S}_{\mathcal{Z}}(R)^{\alpha^{*}} } & \Leftrightarrow \Theta_{x} \otimes_{R} P \otimes_{R} \Theta_{x^{-1}} \simeq P \otimes_{R} R 1_{x}, \text { para todo } x \in G ; \\
& \Leftrightarrow \Theta_{x} \otimes_{R} P \otimes_{R} \Theta_{x^{-1}} \otimes_{R} \Theta_{x} \simeq P \otimes_{R} R 1_{x} \otimes_{R} \Theta_{x}, \text { para todo } x \in G ; \\
& \Leftrightarrow \Theta_{x} \otimes_{R} P \otimes_{R} R 1_{x^{-1}} \simeq P \otimes_{R} \Theta_{x}, \text { para todo } x \in G ; \\
& \Leftrightarrow \Theta_{x} \otimes_{R} R 1_{x^{-1}} \otimes_{R} P \simeq P \otimes_{R} \Theta_{x}, \text { para todo } x \in G ; \\
& \Leftrightarrow \Theta_{x} \otimes_{R} P \simeq P \otimes_{R} \Theta_{x}, \text { para todo } x \in G .
\end{aligned}
$$

\subsection{Produto cruzado generalizado parcial}

Seja

$$
\begin{array}{rlc}
\Theta: \quad G & \longrightarrow & \operatorname{PicS}(R) \\
x & \longrightarrow & {\left[\Theta_{x}\right]}
\end{array}
$$

uma representação parcial unital com $\varepsilon_{x}=\Theta_{x} \otimes \Theta_{x^{-1}} \simeq R 1_{x}$, para todo $x \in G$. Pelo Lema 3.2.1 existe uma família de isomorfismos de $R$-bimódulos

$$
f^{\Theta}=\left\{f_{x, y}^{\Theta}: \Theta_{x} \otimes_{R} \Theta_{y} \longrightarrow 1_{x} \Theta_{x y}, x, y \in G\right\} .
$$

Dizemos que $f^{\Theta}$ é um conjunto de fatores para $\Theta$ se o diagrama abaixo é comutativo

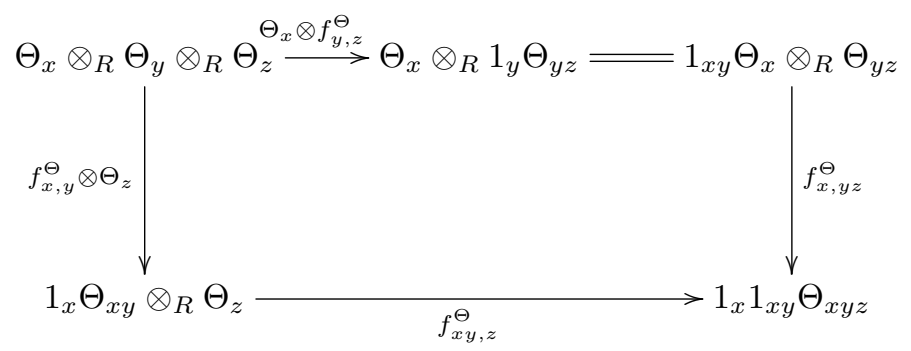


Observação 3.3.1. Se $x=1$, então temos o isomorfismo de R-bimódulos $f_{1, x}^{\Theta}: \Theta_{1} \otimes_{R} \Theta_{y} \longrightarrow R \Theta_{y} \simeq \Theta_{y}$, para todo $y \in G$. Se $y=1$, então $f_{x, 1}^{\Theta}: \Theta_{x} \otimes_{R} \Theta_{1} \longrightarrow 1_{x} \Theta_{x} \simeq \Theta_{x}$ é um isomorfismo de R-bimódulos, para todo $x \in G$.

Se $f^{\Theta}=\left\{f_{x, y}^{\Theta}: \Theta_{x} \otimes_{R} \Theta_{y} \longrightarrow 1_{x} \Theta_{x y}, x, y \in G\right\}$ é um conjunto de fatores para $\Theta$, então $\Delta(\Theta)=$ $\bigoplus_{x \in G} \Theta_{x}$ com a multiplicação

$$
u_{x} \stackrel{\ominus}{\circ} u_{y}=f_{x, y}^{\Theta}\left(u_{x} \otimes u_{y}\right) \in 1_{x} \Theta_{x y}, \quad u_{x} \in \Theta_{x}, u_{y} \in \Theta_{y},
$$

é chamado de produto cruzado generalizado parcial.

Proposição 3.3.2. Sejam $\Theta: G \longrightarrow \operatorname{PicS}(R)$ uma representação parcial unital com $\varepsilon_{x}=\Theta_{x} \otimes_{R} \Theta_{x^{-1}} \simeq$ $R 1_{x}$, para todo $x \in G$, e $f^{\Theta}=\left\{f_{x, y}^{\Theta}: \Theta_{x} \otimes_{R} \Theta_{y} \longrightarrow 1_{x} \Theta_{x y}, x, y \in G\right\}$, um conjunto de fatores para $\Theta$. Então, o produto cruzado generalizado parcial $\Delta(\Theta)$ é um anel associativo com unidade que tem $R \simeq \Theta_{1}$ como subanel.

Demonstração. Como $f_{1,1}^{\Theta}: \Theta_{1} \otimes_{R} \Theta_{1} \longrightarrow \Theta_{1}$, então $\Theta_{1}$ é um subanel de $\Delta(\Theta)$. Por $\Theta$ ser representação parcial, temos $\left[\Theta_{1}\right]=[R]$, assim existe $j: R \longrightarrow \Theta_{1}$ um isomorfismo de $R$-bimódulos. Denote $v=j(1)$, então $\Theta_{1}=j(R)=R v=v R$ e temos

$$
r v=r j(1)=j(r)=j(1) r=v r, \quad \text { para todo } r \in R .
$$

Seja $c \in R$ tal que $f_{1,1}^{\Theta}(v \otimes v)=c v$.

Afirmação 3.3.3. $c \in \mathcal{U}(\mathcal{Z})$.

De fato, seja $r \in R$ tal que $r v=0$, então $0=r v=r j(1)=j(r)$. Como $j$ é isomorfismo devemos ter $r=0$. Seja $r \in R$ qualquer, então

$$
r c v=r f_{1,1}^{\Theta}(v \otimes v)=f_{1,1}^{\Theta}(r v \otimes v)=f_{1,1}^{\Theta}(v \otimes v r)=f_{1,1}^{\Theta}(v \otimes v) r=v c r=c r v,
$$

assim, $(r c-c r) v=0$ o que implica que $r c=c r$, para todo $r \in R$. Logo, $c \in \mathcal{Z}$.

Como $f_{1,1}^{\Theta}$ é isomorfismo, então existe $d \in R$ tal que $\left(f_{1,1}^{\Theta}\right)^{-1}(v)=d v \otimes v$. Então,

$$
d c v=d f_{1,1}^{\Theta}(v \otimes v)=f_{1,1}^{\Theta}(d v \otimes v)=f_{1,1}^{\Theta}\left(f_{1,1}^{\Theta}\right)^{-1}(v)=v .
$$

Logo, $d c=c d=1$. Portanto, $c \in \mathcal{U}(\mathcal{Z})$.

Denote $u=c^{-1} v \in \Theta_{1}$, então $r u=r c^{-1} v=c^{-1} v r=u r$, para todo $r \in R$ e temos

$$
f_{1,1}^{\Theta}(u \otimes u)=f_{1,1}^{\Theta}\left(c^{-1} v \otimes c^{-1} v\right)=c^{-1} f_{1,1}(v \otimes v) c^{-1}=c^{-1} c v c^{-1}=v c^{-1}=u .
$$

Defina $\iota: R \longrightarrow \Theta_{1}$, por $\iota(r)=r u$. Então $\iota$ é um isomorfismo de $R$-bimódulos. Além disso, dados $r, r^{\prime} \in R$ temos

$$
\iota(r) \stackrel{\ominus}{\circ} \iota\left(r^{\prime}\right)=f_{1,1}^{\Theta}\left(r u \otimes r^{\prime} u\right)=r r^{\prime} f_{1,1}(u \otimes u) \stackrel{(3.22)}{=} r r^{\prime} u=\iota\left(r r^{\prime}\right) .
$$

Logo, $\iota$ é isomorfismo de anéis.

Vejamos que $\iota(1)=u \in \Theta_{1}$ é a unidade de $\Delta(\Theta)$. Seja $u_{x} \in \Theta_{x}, x \in G$. Como $f_{1, x}^{\Theta}: \Theta_{1} \otimes_{R} \Theta_{x} \longrightarrow \Theta_{x}$ é um isomorfismo de $R$-bimódulos, então existe $\sum_{i=1}^{n} u r_{i} \otimes u_{x}^{i}=u \otimes \widetilde{u}_{x} \in \Theta_{1} \otimes_{R} \Theta_{x}$, onde $\widetilde{u}_{x}=\sum_{i=1}^{n} r_{i} u_{x}^{i}$, tal que $f_{1, x}^{\Theta}\left(u \otimes \widetilde{u}_{x}\right)=u_{x}$. Então,

$$
\begin{aligned}
\left(u \stackrel{\ominus}{\circ} u_{x}\right) & =f_{1, x}^{\Theta}\left(u \otimes u_{x}\right)=f_{1, x}^{\Theta}\left(u \otimes f_{1, x}^{\Theta}\left(u \otimes \widetilde{u}_{x}\right)\right) \\
& =f_{1, x}^{\Theta}\left(f_{1,1}^{\Theta}(u \otimes u) \otimes \widetilde{u}_{x}\right) \\
& \stackrel{(3.22)}{=} f_{1, x}^{\Theta}\left(u \otimes \widetilde{u}_{x}\right)=u_{x} .
\end{aligned}
$$


Analogamente, como $f_{x, 1}^{\Theta}: \Theta_{x} \otimes_{R} \Theta_{1} \longrightarrow \Theta_{x}$ é isomorfismo, então existe $\overline{u_{x}} \in \Theta_{x}$ tal que $f_{x, 1}^{\Theta}\left(\overline{u_{x}} \otimes u\right)=u_{x}$, então

$$
\begin{aligned}
\left(u_{x} \stackrel{\ominus}{\circ} u\right) & =f_{x, 1}^{\Theta}\left(u_{x} \otimes u\right)=f_{x, 1}^{\Theta}\left(f_{x, 1}^{\Theta}\left(\overline{u_{x}} \otimes u\right) \otimes u\right) \\
& =f_{x, 1}^{\Theta}\left(\overline{u_{x}} \otimes f_{1,1}^{\Theta}(u \otimes u)\right) \\
& =f_{x, 1}^{\Theta}\left(\overline{u_{x}} \otimes u\right)=u_{x} .
\end{aligned}
$$

Portanto, $u$ é unidade para $\Delta(\Theta)$.

Observação 3.3.4. Sejam $\Delta(\Theta)$ um produto cruzado generalizado parcial e $\iota: R \longrightarrow \Theta_{1}$ isomorfismo de $R$-bimódulos e de anéis tal que $\iota(1)=u \in \Theta_{1}$ é unidade para $\Delta(\Theta)$, como na proposição acima. Então os diagramas
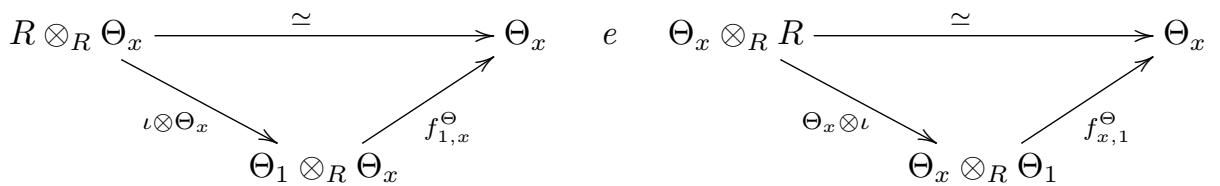

são comutativos. De fato, dados $r \in R$ e $u_{x} \in \Theta_{x}$ temos

$$
\left(f_{1, x}^{\Theta} \circ\left(\iota \otimes \Theta_{x}\right)\right)\left(r \otimes u_{x}\right)=f_{1, x}^{\Theta}\left(\iota(r) \otimes u_{x}\right)=r f_{1, x}^{\Theta}\left(\iota(1) \otimes u_{x}\right)=r u_{x} .
$$

Analogamente,

$$
\left(f_{x, 1}^{\Theta} \circ\left(\Theta_{x} \otimes \iota\right)\right)\left(u_{x} \otimes r\right)=f_{x, 1}\left(u_{x} \otimes \iota(r)\right)=f_{x, 1}\left(u_{x} \otimes \iota(1)\right) r=u_{x} r .
$$

Vamos identificar $R \operatorname{com} \Theta_{1}$, via o isomorfismo de anéis $\iota$, e 1 com $\iota(1)=u \in \Theta_{1}$. Então, $R \subseteq \Delta(\Theta)$ é uma extensão de anéis com mesma unidade.

A partir daqui vamos fixar uma representação parcial unital

$$
\begin{aligned}
\Theta: G & \longrightarrow \operatorname{PicS}(R) \\
x & \longrightarrow\left[\Theta_{x}\right]
\end{aligned}
$$

com $\varepsilon_{x}=\Theta_{x} \otimes_{R} \Theta_{x^{-1}} \simeq R 1_{x}$, para todo $x \in G$. Dado uma família de isomorfismos de $R$-bimódulos $f^{\Theta}=\left\{f_{x, y}^{\Theta}: \Theta_{x} \otimes_{R} \Theta_{y} \longrightarrow 1_{x} \Theta_{x y}, x, y \in G\right\}$, vamos estabelecer uma condição para que $f^{\Theta}$ seja um conjunto de fatores para $\Theta$. Para isso, vamos supor que $f^{\Theta}$ satisfaz os diagramas comutativos (3.23).

Proposição 3.3.5. Seja $f^{\Theta}=\left\{f_{x, y}^{\Theta}: \Theta_{x} \otimes_{R} \Theta_{y} \longrightarrow 1_{x} \Theta_{x y}, x, y \in G\right\}$ uma família de isomorfismos de R-bimódulos que satisfaz os diagramas comutativos dados em (3.23). Dados $x, y, z \in G$ defina o isomorfismo de $R$-bimódulos $\beta_{x, y, z}: 1_{x} 1_{x y} \Theta_{x y z} \longrightarrow 1_{x} 1_{x y} \Theta_{x y z}$ via o diagrama comutativo

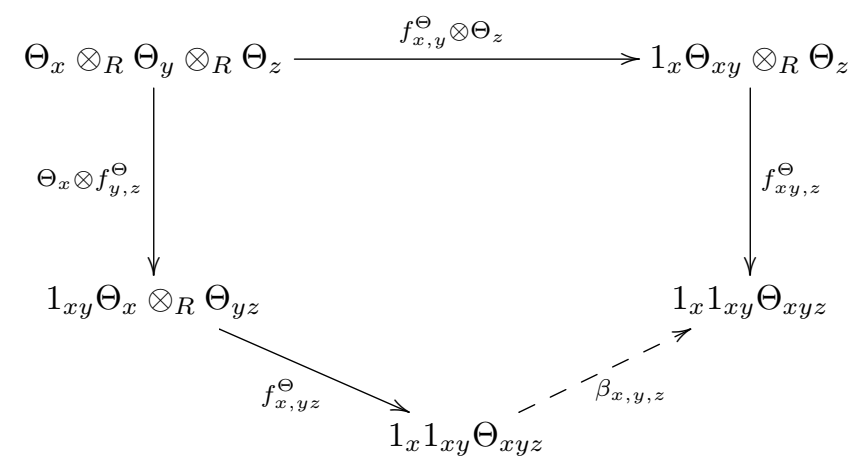


ou seja,

$$
\beta_{x, y, z} \circ f_{x, y z}^{\Theta} \circ\left(\Theta_{x} \otimes f_{y, z}^{\Theta}\right)=f_{x y, z}^{\Theta} \circ\left(f_{x, y}^{\Theta} \otimes \Theta_{z}\right), \forall x, y, z \in G .
$$

Então,

$$
\begin{aligned}
\widetilde{\beta_{-,-,-}: G \times G \times G} & \longrightarrow \mathcal{U}(\mathcal{Z}) \\
(x, y, z) & \longmapsto \widetilde{\beta_{x, y, z}}
\end{aligned}
$$

onde $\widetilde{\beta_{x, y, z}}$ é definida como no Lema 3.2.7, é um elemento normalizado de $Z_{\Theta}^{3}(G, \alpha, \mathcal{Z})$. Além disso, se $\widetilde{\beta_{-,-,-}} \in B_{\Theta}^{3}(G, \alpha, \mathcal{Z})$, ou seja, se existe $\sigma_{-,-}: G \times G \longrightarrow \mathcal{U}(\mathcal{Z})$, com $\sigma_{x, y} \in \mathcal{U}\left(\mathcal{Z} 1_{x} 1_{x y}\right)$ e

$$
\widetilde{\beta_{x, y, z}}=\alpha_{x}\left(\sigma_{y, z} 1_{x^{-1}}\right) \sigma_{x y, z}^{-1} \sigma_{x, y z} \sigma_{x, y}^{-1}, \quad \text { para todo } x, y, z \in G,
$$

então a família de isomorfismos de R-bimódulos

$$
\begin{aligned}
\bar{f}_{x, y}^{\Theta}: \Theta_{x} \otimes_{R} \Theta_{y} & \longrightarrow 1_{x} \Theta_{x, y} \\
u_{x} \otimes u_{y} & \longmapsto \sigma_{x, y} f_{x, y}^{\Theta}\left(u_{x} \otimes u_{y}\right)
\end{aligned}
$$

é um conjunto de fatores para $\Theta$.

Demonstração. Como $\beta_{x, y, z} \in \mathbf{A u t}_{R-R}\left(1_{x} 1_{x y} \Theta_{x y z}\right)$, então pelo Lema 3.2.7, temos que $\widetilde{\beta_{x, y, z}} \in$ $\mathcal{U}\left(\mathcal{Z} 1_{x} 1_{x y} 1_{x y z}\right)$, para todo $x, y, z \in G$. Vamos denotar $f_{x, y}^{\ominus}\left(u_{x} \otimes u_{y}\right)=\left(u_{x} \stackrel{\ominus}{\circ} u_{y}\right)$. Por (3.24), temos

$$
\beta_{x, y, z}\left(\left(u_{x} \stackrel{\ominus}{\circ}\left(u_{y} \stackrel{\ominus}{\circ} u_{z}\right)\right)\right)=\left(\left(u_{x} \stackrel{\ominus}{\circ} u_{y}\right) \stackrel{\ominus}{\circ} u_{z}\right),
$$

para todo $u_{x} \in \Theta_{x}, u_{y} \in \Theta_{y}$ e $u_{z} \in \Theta_{z}$. Como $\left(u_{x} \stackrel{\ominus}{\circ}\left(u_{y} \stackrel{\ominus}{\circ} u_{z}\right)\right) \in 1_{x} 1_{x y} \Theta_{x y z}$, então por (3.17)

$$
\widetilde{\beta_{x, y, z}}\left(u_{x} \stackrel{\ominus}{\circ}\left(u_{y} \stackrel{\ominus}{\circ} u_{z}\right)\right)=\left(\left(u_{x} \stackrel{\ominus}{\circ} u_{y}\right) \stackrel{\ominus}{\circ} u_{z}\right) .
$$

Sejam $x, y, z, t \in G$ e $u_{x} \in \Theta_{x}, u_{y} \in \Theta_{y}, u_{z} \in \Theta_{z}$ e $u_{t} \in \Theta_{z}$. Então,

$$
\begin{aligned}
& \left(\left(\left(u_{x} \stackrel{\ominus}{\circ} u_{y}\right) \stackrel{\ominus}{\circ} u_{z}\right) \stackrel{\ominus}{\circ} u_{t}\right) \stackrel{(3.26)}{=} \widetilde{\beta_{x, y, z}}\left(\left(u_{x} \stackrel{\ominus}{\circ}\left(u_{y} \stackrel{\ominus}{\circ} u_{z}\right)\right) \stackrel{\ominus}{\circ} u_{t}\right) \\
& \stackrel{(3.26)}{=} \widetilde{\beta_{x, y, z}} \widetilde{\beta_{x, y z, t}}\left(u_{x} \stackrel{\ominus}{\circ}\left(\left(u_{y} \stackrel{\ominus}{\circ} u_{z}\right) \stackrel{\ominus}{\circ} u_{t}\right)\right) \\
& \stackrel{(3.26)}{=} \widetilde{\beta_{x, y, z}} \widetilde{\beta_{x, y z, t}}\left(u_{x} \stackrel{\ominus}{\circ} \widetilde{\beta_{y, z, t}}\left(u_{y} \stackrel{\ominus}{\circ}\left(u_{z} \stackrel{\ominus}{\circ} u_{t}\right)\right)\right) \\
& =\widetilde{\beta_{x, y, z}} \widetilde{\beta_{x, y z, t}}\left(u_{x} \widetilde{\beta_{y, z, t}} \stackrel{\ominus}{\circ}\left(u_{y} \stackrel{\ominus}{\circ}\left(u_{z} \stackrel{\ominus}{\circ} u_{t}\right)\right)\right) \\
& \stackrel{(3.14)}{=} \widetilde{\beta_{x, y, z}} \widetilde{\beta_{x, y z, t}} \alpha_{x}\left(\widetilde{\beta_{y, z, t}} 1_{x^{-1}}\right)\left(u_{x} \stackrel{\ominus}{\circ}\left(u_{y} \stackrel{\ominus}{\circ}\left(u_{z} \stackrel{\ominus}{\circ} u_{t}\right)\right)\right)
\end{aligned}
$$

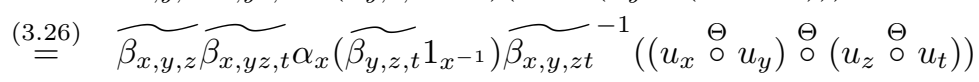

$$
\begin{aligned}
& \stackrel{(3.26)}{=} \underset{\beta_{x, y, z}}{ } \widetilde{\beta_{x, y z, t}} \alpha_{x}\left(\widetilde{\beta_{y, z, t}} 1_{x^{-1}}\right) \widetilde{\beta_{x, y, z t}}-1 \widetilde{\beta_{x y, z, t}}-1\left(\left(\left(u_{x} \stackrel{\ominus}{\circ} u_{y}\right) \stackrel{\ominus}{\circ} u_{z}\right) \stackrel{\ominus}{\circ} u_{t}\right)
\end{aligned}
$$

Assim,

$$
\left(\left(\left(u_{x} \stackrel{\ominus}{\circ} u_{y}\right) \stackrel{\ominus}{\circ} u_{z}\right) \stackrel{\ominus}{\circ} u_{t}\right)=\widetilde{\beta_{x, y, z}} \widetilde{\beta_{x, y z, t}} \alpha_{x}\left(\widetilde{\beta_{y, z, t}} 1_{x^{-1}}\right) \widetilde{\beta_{x, y, z t}}-1 \widetilde{\beta_{x y, z, t}}-1\left(\left(\left(u_{x} \stackrel{\ominus}{\circ} u_{y}\right) \stackrel{\ominus}{\circ} u_{z}\right) \stackrel{\ominus}{\circ} u_{t}\right),
$$

para todo $u_{x} \in \Theta_{x}, u_{y} \in \Theta_{y}, u_{z} \in \Theta_{z}$ e $u_{t} \in \Theta_{z}$.

Considere

$$
1_{x}=\sum_{(x)}\left(\omega_{x} \stackrel{\ominus}{\circ} \omega_{x^{-1}}\right), \quad \omega_{x} \in \Theta_{x}, \omega_{x^{-1}} \in \Theta_{x^{-1}},
$$




$$
\begin{aligned}
1_{y} & =\sum_{(y)}\left(\omega_{y} \stackrel{\ominus}{\circ} \omega_{y^{-1}}\right), \quad \omega_{y} \in \Theta_{y}, \omega_{y^{-1}} \in \Theta_{y^{-1}}, \\
1_{z} & =\sum_{(z}\left(\omega_{z} \stackrel{\ominus}{\circ} \omega_{z^{-1}}\right), \quad \omega_{z} \in \Theta_{z}, \omega_{z^{-1}} \in \Theta_{z^{-1}}, \\
1_{t} & =\sum_{(t)}\left(\omega_{t} \stackrel{\ominus}{\circ} \omega_{t^{-1}}\right), \quad \omega_{t} \in \Theta_{t}, \omega_{t^{-1}} \in \Theta_{t^{-1}} .
\end{aligned}
$$

Então,

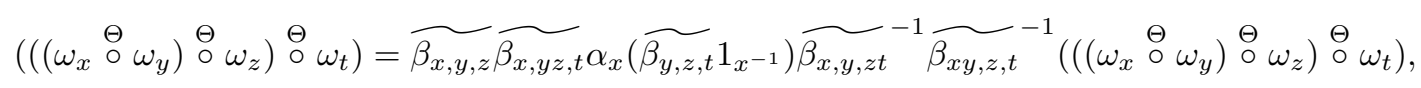

Aplicando $f_{x y z t, t^{-1}}^{\Theta}$, temos:

$\left(\left(\left(\left(\omega_{x} \stackrel{\ominus}{\circ} \omega_{y}\right) \stackrel{\ominus}{\circ} \omega_{z}\right) \stackrel{\ominus}{\circ} \omega_{t}\right) \stackrel{\ominus}{\circ} \omega_{t^{-1}}\right)=\widetilde{\beta_{x, y, z}} \widetilde{\beta_{x, y z, t}} \alpha_{x}\left(\widetilde{\beta_{y, z, t}} 1_{x^{-1}}\right) \widetilde{\beta_{x, y, z t}}-1{\widetilde{\beta_{x y, z, t}}}^{-1}\left(\left(\left(\left(\omega_{x} \stackrel{\ominus}{\circ} \omega_{y}\right) \stackrel{\ominus}{\circ} \omega_{z}\right) \stackrel{\ominus}{\circ} \omega_{t}\right) \stackrel{\ominus}{\circ} \omega_{t-1}\right)$

Por (3.26)

$$
\begin{aligned}
& \beta_{x y z, t, t^{-1}}\left(\left(\left(\omega_{x} \stackrel{\ominus}{\circ} \omega_{y}\right) \stackrel{\ominus}{\circ} \omega_{z}\right) \stackrel{\ominus}{\circ}\left(\omega_{t} \stackrel{\ominus}{\circ} \omega_{t^{-1}}\right)\right) \\
& =\widetilde{\beta}_{\beta_{x, y, z}} \widetilde{\beta_{x, y z, t}} \alpha_{x}\left(\widetilde{\beta_{y, z, t}} 1_{x^{-1}}\right) \widetilde{\beta_{x, y, z t}}-1 \widetilde{\beta_{x y, z, t}}-1 \widetilde{\beta_{x y z, t, t^{-1}}}\left(\left(\left(\omega_{x} \stackrel{\ominus}{\circ} \omega_{y}\right) \stackrel{\ominus}{\circ} \omega_{z}\right) \stackrel{\ominus}{\circ}\left(\omega_{t} \stackrel{\ominus}{\circ} \omega_{t^{-1}}\right)\right)
\end{aligned}
$$

Multiplicando por ${\widetilde{\beta_{x y z, t, t^{-1}}}}^{-1}$ :

$$
\begin{aligned}
& 1_{x y z} 1_{x y z t}\left(\left(\left(\omega_{x} \stackrel{\ominus}{\circ} \omega_{y}\right) \stackrel{\ominus}{\circ} \omega_{z}\right) \stackrel{\ominus}{\circ}\left(\omega_{t} \stackrel{\ominus}{\circ} \omega_{t-1}\right)\right) \\
& =\widetilde{\beta_{x, y, z}} \widetilde{\beta_{x, y z, t}} \alpha_{x}\left(\widetilde{\beta_{y, z, t}} 1_{x^{-1}}\right) \widetilde{\beta_{x, y, z t}}-1{\widetilde{\beta_{x y, z, t}}}^{-1} 1_{x y z} 1_{x y z t}\left(\left(\left(\omega_{x} \stackrel{\ominus}{\circ} \omega_{y}\right) \stackrel{\ominus}{\circ} \omega_{z}\right) \stackrel{\ominus}{\circ}\left(\omega_{t} \stackrel{\ominus}{\circ} \omega_{t^{-1}}\right)\right)
\end{aligned}
$$

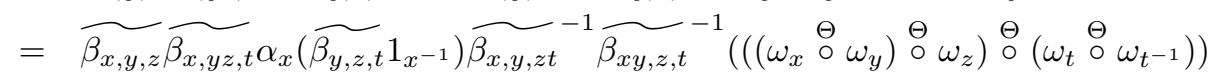

Somando em $(t)$ e usando que o diagrama (3.23) é comutativo:

$$
\begin{aligned}
& \sum_{(t)} 1_{x y z} 1_{x y z t}\left(\left(\left(\omega_{x} \stackrel{\ominus}{\circ} \omega_{y}\right) \stackrel{\ominus}{\circ} \omega_{z}\right) \stackrel{\ominus}{\circ}\left(\omega_{t} \stackrel{\ominus}{\circ} \omega_{t-1}\right)\right) \\
& =\sum_{(t)}{\widetilde{\beta_{x, y, z}}}_{\beta_{x, y z, t}} \alpha_{x}\left({\widetilde{\beta_{y, z, t}}}_{x^{-1}}\right){\widetilde{\beta_{x, y, z t}}}^{-1}{\widetilde{\beta_{x y, z, t}}}^{-1}\left(\left(\left(\omega_{x} \stackrel{\ominus}{\circ} \omega_{y}\right) \stackrel{\ominus}{\circ} \omega_{z}\right) \stackrel{\ominus}{\circ}\left(\omega_{t} \stackrel{\ominus}{\circ} \omega_{t^{-1}}\right)\right)
\end{aligned}
$$

$$
\begin{aligned}
& 1_{x y z} 1_{x y z t}\left(\left(\left(\omega_{x} \stackrel{\ominus}{\circ} \omega_{y}\right) \stackrel{\ominus}{\circ} \omega_{z}\right)\right) 1_{t}=\widetilde{\beta_{x, y, z}} \widetilde{\beta_{x, y z, t}} \alpha_{x}\left(\widetilde{\beta_{y, z, t}} 1_{x^{-1}}\right) \widetilde{\beta_{x, y, z t}}-1{\widetilde{\beta_{x y, z, t}}}^{-1}\left(\left(\left(\omega_{x} \stackrel{\ominus}{\circ} \omega_{y}\right) \stackrel{\ominus}{\circ} \omega_{z}\right)\right) 1_{t}
\end{aligned}
$$

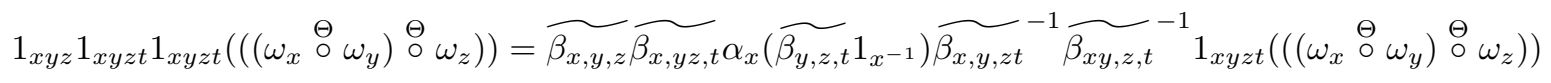

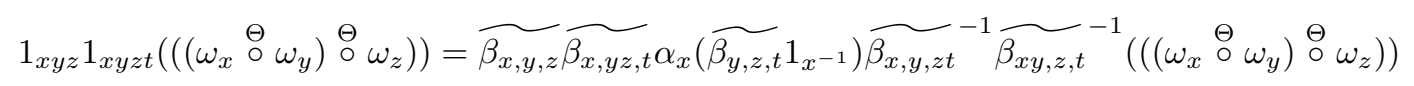

Analogamente, aplicando $f_{x y z, z^{-1}}^{\Theta}$, multiplicando por $\widetilde{\beta_{x y, z, z^{-1}}}$ e somando em $(t)$ :

$$
\begin{aligned}
& \sum_{(z)}{\widetilde{\beta_{x y, z, z^{-1}}}}^{-1} 1_{x y z} 1_{x y z t}\left(\left(\left(\omega_{x} \stackrel{\ominus}{\circ} \omega_{y}\right) \stackrel{\ominus}{\circ} \omega_{z}\right) \stackrel{\ominus}{\circ} \omega_{z^{-1}}\right) \\
= & \sum_{(z)}{\widetilde{\beta_{x y, z, z^{-1}}}}^{-1}{\widetilde{\beta_{x, y, z}}}_{\beta_{x, y z, t}} \alpha_{x}\left(\widetilde{\beta_{y, z, t}} 1_{x^{-1}}\right){\widetilde{\beta_{x, y, z t}}}^{-1}{\widetilde{\beta_{x y, z, t}}}^{-1}\left(\left(\left(\omega_{x} \stackrel{\ominus}{\circ} \omega_{y}\right) \stackrel{\ominus}{\circ} \omega_{z}\right) \stackrel{\ominus}{\circ} \omega_{z^{-1}}\right)
\end{aligned}
$$


Por (3.26),

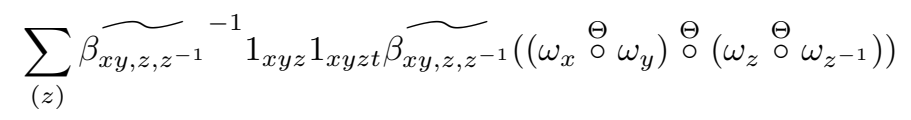

$$
\begin{aligned}
& =\sum_{(z)}{\widetilde{\beta_{x y, z, z^{-1}}}}^{-1} \widetilde{\beta_{x, y, z}} \widetilde{\beta_{x, y z, t}} \alpha_{x}\left(\widetilde{\beta_{y, z, t}} 1_{x^{-1}}\right) \widetilde{\beta_{x, y, z t}}-1 \widetilde{\beta_{x y, z, t}}-1 \underset{\beta_{x y, z, z^{-1}}}{\widetilde{\sim}}\left(\left(\omega_{x} \stackrel{\ominus}{\circ} \omega_{y}\right) \stackrel{\ominus}{\circ}\left(\omega_{z} \stackrel{\ominus}{\circ} \omega_{z^{-1}}\right)\right) \\
& \sum_{(z)} 1_{x y z} 1_{x y z t} 1_{x y} 1_{x y z}\left(\left(\omega_{x} \stackrel{\ominus}{\circ} \omega_{y}\right) \stackrel{\ominus}{\circ}\left(\omega_{z} \stackrel{\ominus}{\circ} \omega_{z^{-1}}\right)\right) \\
& =\sum_{(z)} \widetilde{\beta_{x, y, z}} \widetilde{\beta_{x, y z, t}} \alpha_{x}\left(\widetilde{\beta_{y, z, t}} 1_{x^{-1}}\right) \widetilde{\beta_{x, y, z t}}-1{\widetilde{\beta_{x y, z, t}}}^{-1} 1_{x y} 1_{x y z}\left(\left(\omega_{x} \stackrel{\ominus}{\circ} \omega_{y}\right) \stackrel{\ominus}{\circ}\left(\omega_{z} \stackrel{\ominus}{\circ} \omega_{z^{-1}}\right)\right) \\
& \sum_{(z)} 1_{x y} 1_{x y z} 1_{x y z t}\left(\left(\omega_{x} \stackrel{\ominus}{\circ} \omega_{y}\right) \stackrel{\ominus}{\circ}\left(\omega_{z} \stackrel{\ominus}{\circ} \omega_{z^{-1}}\right)\right) \\
& =\sum_{(z)} \widetilde{\beta_{x, y, z}} \widetilde{\beta_{x, y z, t}} \alpha_{x}\left(\widetilde{\beta_{y, z, t}} 1_{x^{-1}}\right) \widetilde{\beta_{x, y, z t}}-1{\widetilde{\beta_{x y, z, t}}}^{-1}\left(\left(\omega_{x} \stackrel{\ominus}{\circ} \omega_{y}\right) \stackrel{\ominus}{\circ}\left(\omega_{z} \stackrel{\ominus}{\circ} \omega_{z^{-1}}\right)\right) \\
& 1_{x y} 1_{x y z} 1_{x y z t}\left(\omega_{x} \stackrel{\ominus}{\circ} \omega_{y}\right) 1_{z}=\widetilde{\beta_{x, y, z}} \widetilde{\beta_{x, y z, t}} \alpha_{x}\left(\widetilde{\beta_{y, z, t}} 1_{x^{-1}}\right) \widetilde{\beta_{x, y, z t}}-1 \widetilde{\beta_{x y, z, t}}-1\left(\omega_{x} \stackrel{\ominus}{\circ} \omega_{y}\right) 1_{z} \\
& 1_{x y} 1_{x y z} 1_{x y z t} 1_{x y z}\left(\omega_{x} \stackrel{\ominus}{\circ} \omega_{y}\right)=\widetilde{\beta_{x, y, z}} \widetilde{\beta_{x, y z, t}} \alpha_{x}\left(\widetilde{\beta_{y, z, t}} 1_{x^{-1}}\right) \widetilde{\beta_{x, y, z t}}-1 \widetilde{\beta_{x y, z, t}}-11_{x y z}\left(\omega_{x} \stackrel{\ominus}{\circ} \omega_{y}\right) \\
& 1_{x y} 1_{x y z} 1_{x y z t}\left(\omega_{x} \stackrel{\ominus}{\circ} \omega_{y}\right)=\widetilde{\beta_{x, y, z}} \widetilde{\beta_{x, y z, t}} \alpha_{x}\left(\widetilde{\beta_{y, z, t}} 1_{x^{-1}}\right) \widetilde{\beta_{x, y, z t}}{\widetilde{\beta_{x y, z, t}}}^{-1}\left(\omega_{x} \stackrel{\ominus}{\circ} \omega_{y}\right)
\end{aligned}
$$

Aplicando $f_{x y, y^{-1}}^{\Theta}$, multiplicando por ${\widetilde{\beta_{x, y, y^{-1}}}}^{-1}$ e somando em $(y)$ :

$$
\begin{aligned}
& \sum_{(y)}{\widetilde{\beta_{x, y, y^{-1}}}}^{-1} 1_{x y} 1_{x y z} 1_{x y z t}\left(\left(\omega_{x} \stackrel{\ominus}{\circ} \omega_{y}\right) \stackrel{\ominus}{\circ} \omega_{y^{-1}}\right) \\
= & \sum_{(y)}{\widetilde{\beta_{x, y, y^{-1}}}}^{-1} \widetilde{\beta_{x, y, z}} \widetilde{\beta_{x, y z, t}} \alpha_{x}\left(\widetilde{\beta_{y, z, t}} 1_{x^{-1}}\right) \widetilde{\beta_{x, y, z t}}-1 \widetilde{\beta_{x y, z, t}}-1\left(\left(\omega_{x} \stackrel{\ominus}{\circ} \omega_{y}\right) \stackrel{\ominus}{\circ} \omega_{y^{-1}}\right)
\end{aligned}
$$

Por (3.26),

$$
\begin{aligned}
& \sum_{(y)}{\widetilde{\beta_{x, y, y^{-1}}}}^{-1} 1_{x y} 1_{x y z} 1_{x y z t} \widetilde{\beta_{x, y, y^{-1}}}\left(\omega_{x} \stackrel{\ominus}{\circ}\left(\omega_{y} \stackrel{\ominus}{\circ} \omega_{y^{-1}}\right)\right) \\
& =\sum_{(y)}{\widetilde{\beta_{x, y, y^{-1}}}}^{-1} \widetilde{\beta_{x, y, z}} \widetilde{\beta_{x, y z, t}} \alpha_{x}\left(\widetilde{\beta_{y, z, t}} 1_{x^{-1}}\right) \widetilde{\beta_{x, y, z t}}-1{\widetilde{\beta_{x y, z, t}}}^{-1} \widetilde{\beta_{x, y, y^{-1}}}\left(\omega_{x} \stackrel{\ominus}{\circ}\left(\omega_{y} \stackrel{\ominus}{\circ} \omega_{y^{-1}}\right)\right) \\
& \sum_{(y)} 1_{x y} 1_{x y z} 1_{x y z t} 1_{x} 1_{x y}\left(\omega_{x} \stackrel{\ominus}{\circ}\left(\omega_{y} \stackrel{\ominus}{\circ} \omega_{y^{-1}}\right)\right) \\
& =\sum_{(y)} \widetilde{\beta_{x, y, z}} \widetilde{\beta_{x, y z, t}} \alpha_{x}\left(\widetilde{\beta_{y, z, t}} 1_{x^{-1}}\right) \widetilde{\beta_{x, y, z t}}-1 \widetilde{\beta_{x y, z, t}}-11_{x} 1_{x y}\left(\omega_{x} \stackrel{\ominus}{\circ}\left(\omega_{y} \stackrel{\ominus}{\circ} \omega_{y^{-1}}\right)\right) \\
& 1_{x} 1_{x y} 1_{x y z} 1_{x y z t} \omega_{x} 1_{y}=\widetilde{\beta_{x, y, z}} \widetilde{\beta_{x, y z, t}} \alpha_{x}\left(\widetilde{\beta_{y, z, t}} 1_{x^{-1}}\right) \widetilde{\beta_{x, y, z t}}-1{\widetilde{\beta_{x y, z, t}}}^{-1} \omega_{x} 1_{y} \\
& 1_{x} 1_{x y} 1_{x y z} 1_{x y z t} 1_{x y} \omega_{x}=\widetilde{\beta_{x, y, z}} \widetilde{\beta_{x, y z, t}} \alpha_{x}\left(\widetilde{\beta_{y, z, t}} 1_{x^{-1}}\right) \widetilde{\beta_{x, y, z t}}{\widetilde{\beta_{x y, z, t}}}^{-1} 1_{x y} \omega_{x}
\end{aligned}
$$




$$
1_{x} 1_{x y} 1_{x y z} 1_{x y z t} \omega_{x}=\widetilde{\beta_{x, y, z}} \widetilde{\beta_{x, y z, t}} \alpha_{x}\left(\widetilde{\beta_{y, z, t}} 1_{x^{-1}}\right){\widetilde{\beta_{x, y, z t}}}^{-1}{\widetilde{\beta_{x y, z, t}}}^{-1} \omega_{x}
$$

Por fim, apliando $f_{x, x^{-1}}^{\Theta}$ e somando em $(x)$ :

$$
\begin{aligned}
& \sum_{(x)} 1_{x} 1_{x y} 1_{x y z} 1_{x y z t}\left(\omega_{x} \stackrel{\ominus}{\circ} \omega_{x^{-1}}\right)=\sum_{(x)} \widetilde{\beta_{x, y, z}} \widetilde{\beta_{x, y z, t}} \alpha_{x}\left(\widetilde{\beta_{y, z, t}} 1_{x^{-1}}\right) \widetilde{\beta_{x, y, z t}}-1{\widetilde{\beta_{x y, z, t}}}^{-1}\left(\omega_{x} \stackrel{\ominus}{\circ} \omega_{x^{-1}}\right) \\
& 1_{x} 1_{x y} 1_{x y z} 1_{x y z t} 1_{x}=\widetilde{\beta_{x, y, z}} \widetilde{\beta_{x, y z, t}} \alpha_{x}\left(\widetilde{\beta_{y, z, t}} 1_{x^{-1}}\right){\widetilde{\beta_{x, y, z t}}}^{-1}{\widetilde{\beta_{x y, z, t}}}^{-1} 1_{x} \\
& 1_{x} 1_{x y} 1_{x y z} 1_{x y z t}=\widetilde{\beta_{x, y, z}} \widetilde{\beta_{x, y z, t}} \alpha_{x}\left(\widetilde{\beta_{y, z, t}} 1_{x^{-1}}\right){\widetilde{\beta_{x, y, z t}}}^{-1} \widetilde{\beta_{x y, z, t}}-1, \quad \forall x, y, z, t \in G . \\
& \alpha_{x}\left(\widetilde{\beta_{y, z, t}} 1_{x^{-1}}\right) \widetilde{\beta_{x, y, z}} \widetilde{\beta_{x, y z, t}}=\widetilde{\beta_{x, y, z t}} \widetilde{\beta_{x y, z, t}}, \forall x, y, z, t \in G .
\end{aligned}
$$

Logo, $\widetilde{\beta_{-,-,-}} \in Z_{\Theta}^{3}(G, \alpha, \mathcal{Z})$.

Vejamos que $\widetilde{\beta_{-,-,-}}$é normalizado. Seja $x=1$, por (3.26) e pela comutatividade dos diagramas em

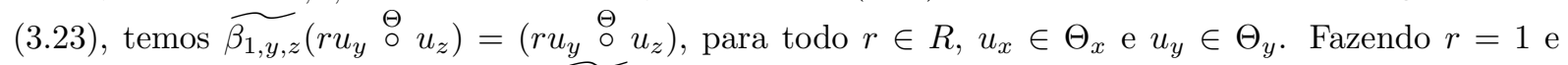
seguindo o argumento acima, temos $\widetilde{\beta_{1, y, z}}=1_{y} 1_{y z}$. Analogamente, temos

$$
\widetilde{\beta_{x, 1, z}}=1_{x} 1_{x z} \text { e } \widetilde{\beta_{x, y, 1}}=1_{x} 1_{x y}, \text { para todos } x, y, z \in G .
$$

Portanto, $\widetilde{\beta_{-,-,-}}$é um elemento normalizado em $Z_{\Theta}^{3}(G, \alpha, \mathcal{Z})$.

Vamos verificar que $\bar{f}^{\Theta}=\left\{\bar{f}_{x, y}^{\Theta}, x, y \in G\right\}$ é um conjunto de fatores para $\Theta$. Sejam $x, y, z \in G$ e $u_{x} \in \Theta_{x}, u_{y} \in \Theta_{y}, u_{z} \in \Theta_{z}$, então

$$
\begin{aligned}
& \bar{f}_{x, y z}^{\ominus}\left(u_{x} \otimes \bar{f}_{y, z}^{\ominus}\left(u_{y} \otimes u_{z}\right)\right)=\sigma_{x, y z}\left(u_{x} \stackrel{\ominus}{\circ} \sigma_{y, z}\left(u_{y} \stackrel{\ominus}{\circ} u_{z}\right)\right) \\
& =\sigma_{x, y z}\left(u_{x} \sigma_{y, z} \stackrel{\ominus}{\circ}\left(u_{y} \stackrel{\ominus}{\circ} u_{z}\right)\right) \\
& \stackrel{(3.14)}{=} \sigma_{x, y z}\left(\alpha_{x}\left(\sigma_{y, z} 1_{x^{-1}}\right) u_{x} \stackrel{\ominus}{\circ}\left(u_{y} \stackrel{\ominus}{\circ} u_{z}\right)\right) \\
& =\sigma_{x, y z} \alpha_{x}\left(\sigma_{y, z} 1_{x^{-1}}\right)\left(u_{x} \stackrel{\ominus}{\circ}\left(u_{y} \stackrel{\ominus}{\circ} u_{z}\right)\right) \\
& =\sigma_{x, y z} \alpha_{x}\left(\sigma_{y, z} 1_{x^{-1}}\right) \widetilde{\beta_{x, y, z}}\left(\left(u_{x} \stackrel{\ominus}{\circ} u_{y}\right) \stackrel{\ominus}{\circ} u_{z}\right) \\
& \stackrel{(3.25)}{=} \sigma_{x y, z} \sigma_{x, y z}\left(\left(u_{x} \stackrel{\ominus}{\circ} u_{y}\right) \stackrel{\ominus}{\circ} u_{z}\right) \\
& =\sigma_{x y, z}\left(\sigma_{x, y z}\left(u_{x} \stackrel{\ominus}{\circ} u_{y}\right) \stackrel{\ominus}{\circ} u_{z}\right) \\
& =\bar{f}_{x y, z}^{\Theta}\left(\bar{f}_{x, y}^{\Theta}\left(u_{x} \otimes u_{y}\right) \otimes u_{z}\right) .
\end{aligned}
$$

Portanto, $\bar{f}^{\Theta}=\left\{\bar{f}_{x, y}^{\Theta}, x, y \in G\right\}$ é um conjunto de fatores para $\Theta$.

Corolário 3.3.6. Seja $\Gamma: G \longrightarrow \operatorname{PicS}(R)$ uma representação parcial unital com $\Gamma_{x} \otimes_{R} \Gamma_{x^{-1}} \simeq R 1_{x} e$ $\Gamma_{x} \mid \Theta_{x}$, para todo $x \in G$. Sejam $f^{\Gamma}=\left\{f_{x, y}^{\Gamma}: \Gamma_{x} \otimes \Gamma_{y} \longrightarrow 1_{x} \Gamma_{x y}, \in x, y \in G\right\}$ uma família de isomorfismos de $R$-bimódulos que satisfaz os diagramas comutativos em (3.23) e $\beta_{x, y, z}^{\Gamma}: 1_{x} 1_{x y} \Theta_{x y z} \longrightarrow 1_{x} 1_{x y} \Theta_{x y z}$ isomorfismo de R-bimódulos que satisfaz

$$
\beta_{x, y, z}^{\Gamma} \circ f_{x, y z}^{\Gamma} \circ\left(\Theta_{x} \otimes f_{y, z}^{\Gamma}\right)=f_{x y, z}^{\Gamma} \circ\left(f_{x, y}^{\Gamma} \otimes \Theta_{z}\right), \forall x, y, z \in G .
$$

Então $\widetilde{\beta_{-,-,-}^{\Gamma}} \in Z_{\Theta}^{3}(G, \alpha, \mathcal{Z})$. 
Demonstração. Segue da demonstração da Proposição 3.3.5 e de (3.15).

O próximo resultado garante a unicidade da classe do 3-cociclo dado no Corolário 3.3.6 em $H_{\Theta}^{3}(G, \alpha, \mathcal{Z})$.

Proposição 3.3.7. Seja $\Gamma: G \longrightarrow \operatorname{PicS}(R)$ uma representação parcial unital com $\Gamma_{x} \otimes_{R} \Gamma_{x^{-1}} \simeq R 1_{x} e$ $\Gamma_{x} \mid \Theta_{x}$, para todo $x \in G$, e $f^{\Gamma}=\left\{f_{x, y}^{\Gamma}: \Gamma_{x} \otimes_{R} \Gamma_{y} \longrightarrow 1_{x} \Gamma_{x y}, x, y \in G\right\}$ uma família de isomorfismos de $R$-bimódulos que satisfaz os diagramas comutativos em (3.23). Então a classe $\left[\widetilde{\left.\beta_{-,-,-}^{\Gamma}\right]}\right.$ em $H_{\Theta}^{3}(G, \alpha, \mathcal{Z})$ não depende da escolha de representante em $\left[\Gamma_{x}\right]$ e nem da família de isomorfismos de R-bimódulos $f^{\Gamma}$.

Demonstração. Seja $\Omega_{x} \in\left[\Gamma_{x}\right]$, então existe um isomorfismo de $R$-bimódulos $a_{x}: \Omega_{x} \longrightarrow \Gamma_{x}$, para todo $x \in G$. Sejam

$$
f^{\Omega}=\left\{f_{x, y}^{\Omega}: \Omega_{x} \otimes_{R} \Omega_{y} \longrightarrow 1_{x} \Omega_{x y}, x, y \in G\right\}, f^{\Gamma}=\left\{f_{x, y}^{\Gamma}: \Gamma_{x} \otimes_{R} \Gamma_{y} \longrightarrow 1_{x} \Gamma_{x y}, x, y \in G\right\}
$$

família de isomorfismos de $R$-bimódulos que satisfazem os diagramas comutativos em (3.23) e $\widetilde{\beta_{-,-,-}^{\Omega}}$ e $\widetilde{\beta_{-,-,-}^{\Gamma}}$ os 3 -cociclos associados a $f^{\Omega}$ e $f^{\Gamma}$, respectivamente. Por definição temos que

$$
\begin{aligned}
& \beta_{x, y, z}^{\Omega} \circ f_{x, y z}^{\Omega} \circ\left(\Omega_{x} \otimes f_{y, z}^{\Omega}\right)=f_{x y, z}^{\Omega} \circ\left(f_{x, y}^{\Omega} \otimes \Omega_{z}\right), \\
& \beta_{x, y, z}^{\Gamma} \circ f_{x, y z}^{\Gamma} \circ\left(\Gamma_{x} \otimes f_{y, z}^{\Gamma}\right)=f_{x y, z}^{\Gamma} \circ\left(f_{x, y}^{\Gamma} \otimes \Gamma_{z}\right) .
\end{aligned}
$$

Seja $b_{x, y}: 1_{x} \Omega_{x y} \longrightarrow 1_{x} \Omega_{x y}$ o isomorfismo de $R$-bimódulos definido via o diagrama comutativo

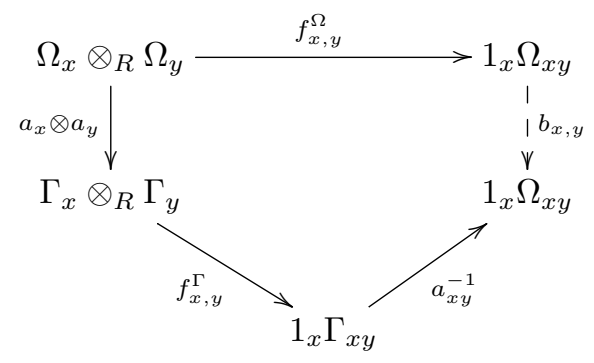

ou seja,

$$
a_{x y} \circ b_{x, y} \circ f_{x, y}^{\Omega}=f_{x, y}^{\Gamma} \circ\left(a_{x} \otimes a_{y}\right), \quad \forall x, y \in G .
$$

Seja $\widetilde{b_{x, y}} \in \mathcal{U}\left(\mathcal{Z} 1_{x} 1_{x y}\right)$ a imagem de $b_{x, y}$ via o isomorfismo do Lema 3.2.7. Por (3.17) temos $\widetilde{b_{x, y}} t_{x y}=$ $b_{x, y}\left(1_{x} t_{x y}\right)$, para todo $t_{x y} \in \Omega_{x y}$. Denotando $f_{x, y}^{\Omega}\left(t_{x} \otimes t_{y}\right)=\left(t_{x} \stackrel{\Omega}{\circ} t_{y}\right)$ e $f_{x, y}^{\Gamma}\left(v_{x} \otimes v_{y}\right)=\left(v_{x} \stackrel{\Gamma}{\circ} v_{y}\right)$, temos

$$
\begin{aligned}
& \widetilde{\beta_{x, y, z}^{\Omega}}\left(t_{x} \stackrel{\Omega}{\circ}\left(t_{y} \stackrel{\Omega}{\circ} t_{z}\right)\right)=\left(\left(t_{x} \stackrel{\Omega}{\circ} t_{y}\right) \stackrel{\Omega}{\circ} t_{z}\right), \\
& \widetilde{\beta_{x, y, z}^{\Gamma}}\left(v_{x} \stackrel{\Gamma}{\circ}\left(g_{y} \circ v_{z}\right)\right)=\left(\left(v_{x} \circ v_{y}\right) \stackrel{\Gamma}{\circ} v_{z}\right), \\
& \widetilde{b_{x, y}} a_{x y}\left(t_{x} \stackrel{\Omega}{\circ} t_{y}\right)=\left(a_{x}\left(t_{x}\right) \circ a_{y}\left(t_{y}\right)\right) .
\end{aligned}
$$


Dados $x, y, z \in G$ e $t_{x} \in \Omega_{x}, t_{y} \in \Omega_{y}, t_{z} \in \Omega_{z}$ temos:

$$
\begin{aligned}
& a_{x y z}\left(\left(t_{x} \stackrel{\Omega}{\circ} t_{y}\right) \stackrel{\Omega}{\circ} t_{z}\right) \stackrel{(3.29)}{=} \widetilde{b_{x y, z}^{-1}}\left(a_{x y}\left(t_{x} \stackrel{\Omega}{\circ} t_{y}\right) \stackrel{\Gamma}{\circ} a_{z}\left(t_{z}\right)\right) \\
& \stackrel{(3.29)}{=} \widetilde{b_{x y, z}^{-1} \widetilde{b_{x, y}^{-1}}}\left(\left(a_{x}\left(t_{x}\right) \stackrel{\Gamma}{\circ} a_{y}\left(t_{y}\right)\right) \stackrel{\Gamma}{\circ} a_{z}\left(t_{z}\right)\right) \\
& \stackrel{(3.28)}{=} \widetilde{b_{x y, z}^{-1}} \widetilde{b_{x, y}^{-1}} \widetilde{\beta_{x, y, z}^{\Gamma}}\left(a_{x}\left(t_{x}\right) \stackrel{\Gamma}{\circ}\left(a_{y}\left(t_{y}\right) \stackrel{\Gamma}{\circ} a_{z}\left(t_{z}\right)\right)\right) \\
& \stackrel{(3.29)}{=} \widetilde{b_{x y, z}^{-1} b_{x, y}^{-1}} \widetilde{\beta_{x, y, z}^{\Gamma}}\left(a_{x}\left(t_{x}\right) \stackrel{\Gamma}{\circ} \widetilde{b_{y, z}} a_{y z}\left(t_{y} \stackrel{\Omega}{\circ} t_{z}\right)\right) \\
& \stackrel{(3.14)}{=} \widetilde{b_{x y, z}^{-1} b_{x, y}^{-1}} \widetilde{\beta_{x, y, z}^{\Gamma}} \alpha_{x}\left(\widetilde{b_{y, z}} 1_{x^{-1}}\right)\left(a_{x}\left(t_{x}\right) \stackrel{\Gamma}{\circ} a_{y z}\left(t_{y} \stackrel{\Omega}{\circ} t_{z}\right)\right) \\
& \stackrel{(3.29)}{=} \widetilde{b_{x y, z}^{-1} b_{x, y}^{-1}} \widetilde{\beta_{x, y, z}^{\Gamma}} \alpha_{x}\left(\widetilde{b_{y, z}} 1_{x^{-1}}\right) \widetilde{b_{x, y z}} a_{x y z}\left(t_{x} \stackrel{\Omega}{\circ}\left(t_{y} \stackrel{\Omega}{\circ} t_{z}\right)\right) \\
& \stackrel{(3.27)}{=} \widetilde{b_{x y, z}^{-1} b_{x, y}^{-1}} \widetilde{\beta_{x, y, z}^{\Gamma}} \alpha_{x}\left(\widetilde{b_{y, z}} 1_{x^{-1}}\right) \widetilde{b_{x, y z}} \widetilde{\beta_{x, y, z}^{\Omega}}-1 a_{x y z}\left(\left(t_{x} \stackrel{\Omega}{\circ} t_{y}\right) \stackrel{\Omega}{\circ} t_{z}\right) \\
& =a_{x y z}\left(\widetilde{b_{x y, z}^{-1} \widetilde{b_{x, y}^{-1}} \widetilde{\beta_{x, y, z}^{\Gamma}} \alpha_{x}\left(\widetilde{b_{y, z}} 1_{x^{-1}}\right) \widetilde{b_{x, y z}} \widetilde{\beta_{x, y, z}^{\Omega}}-1}\left(\left(t_{x} \stackrel{\Omega}{\circ} t_{y}\right) \stackrel{\Omega}{\circ} t_{z}\right)\right) \text {. }
\end{aligned}
$$

Como $a_{x y z}$ é isomorfismo segue que

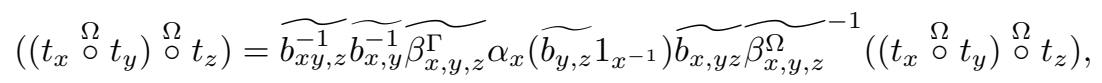

para todo $t_{x} \in \Omega_{x}, t_{y} \in \Omega_{y}, t_{z} \in \Omega_{z}$. Usando o mesmo argumento da Proposição 3.3 .5 obtemos que:

$$
\widetilde{b_{x y, z}^{-1} b_{x, y}^{-1} \widetilde{\beta_{x, y, z}^{\Gamma}} \alpha_{x}\left(\widetilde{b_{y, z}} 1_{x^{-1}}\right) \widetilde{b_{x, y z}} \widetilde{\beta_{x, y, z}^{\Omega}}=1}=1_{x} 1_{x y} 1_{x y z} .
$$

Logo,

$$
\begin{aligned}
\widetilde{\beta_{x, y, z}^{\Omega}} & =\widetilde{\beta_{x, y, z}^{\Gamma}} \widetilde{b_{x y, z}^{-1}} \widetilde{b_{x, y}^{-1}} \alpha_{x}\left(\widetilde{b_{y, z}} 1_{x^{-1}}\right) \widetilde{b_{x, y z}} \\
& =\widetilde{\beta_{x, y, z}^{\Gamma}}\left(\delta^{2} \widetilde{b_{-,-}}\right)(x, y, z),
\end{aligned}
$$

onde

$$
\begin{aligned}
\widetilde{b_{-,-}}: G \times G & \longrightarrow \mathcal{U}(\mathcal{Z}) \\
(x, y) & \longmapsto \widetilde{b_{x, y}} \in \mathcal{U}\left(\mathcal{Z} 1_{x} 1_{x y}\right) .
\end{aligned}
$$

Portanto, $\left[\widetilde{\beta_{-,-,-}^{\Omega}}\right]=\left[\widetilde{\beta_{-,-,-}^{\Gamma}}\right]$ em $H_{\Theta}^{3}(G, \alpha, \mathcal{Z})$

O próximo lema é um resultado técnico que mostra como construir uma representação parcial unital a partir de outras dadas e como relacionar os 3-cociclos associados a essas representações. Em particular, mostramos como construir um produto cruzado generalizado parcial a partir de outros produtos dados.

Lema 3.3.8. Sejam $\Theta, \Omega, \Gamma: G \longrightarrow \operatorname{PicS}(R)$ representações parciais unitais com $\Gamma_{x}\left|\Theta_{x}, \Omega_{x}\right| \Theta_{x} e$ $\Omega_{x} \otimes_{R} \Omega_{x^{-1}} \simeq \Gamma_{x} \otimes_{R} \Gamma_{x^{-1}} \simeq \Theta_{x} \otimes_{R} \Theta_{x^{-1}} \simeq R 1_{x}$, para todo $x \in G$. Seja $f^{\Theta}=\left\{f_{x, y}^{\Theta}: \Theta_{x} \otimes_{R} \Theta_{y} \longrightarrow\right.$ $\left.1_{x} \Theta_{x y}, x, y \in G\right\}$ um conjunto de fatores para $\Theta e$

$$
f^{\Gamma}=\left\{f_{x, y}^{\Gamma}: \Gamma_{x} \otimes_{R} \Gamma_{y} \longrightarrow 1_{x} \Gamma_{x y}, x, y \in G\right\}, f^{\Omega}=\left\{f_{x, y}^{\Omega}: \Omega_{x} \otimes_{R} \Omega_{y} \longrightarrow 1_{x} \Omega_{x y}, x, y \in G\right\}
$$

famílias de isomorfismos de R-bimodulos que satisfazem os diagramas comutativos em (3.23) e $\widehat{\beta_{-,-,-}^{\Gamma-}} e$ $\widetilde{\beta_{-,-,-}^{\Omega}}$ os 3-cociclos em $Z_{\Theta}^{3}(G, \alpha, \mathcal{Z})$ associados a $f^{\Gamma}$ e $f^{\Omega}$, respectivamente. Então,

$$
\begin{aligned}
\Lambda: \quad G & \longrightarrow \operatorname{PicS}(R) \\
x & \longmapsto\left[\Gamma_{x} \otimes_{R} \Theta_{x^{-1}} \otimes_{R} \Omega_{x}\right]
\end{aligned}
$$


é uma representação parcial unital com $\Lambda_{x} \otimes_{R} \Lambda_{x^{-1}} \simeq R 1_{x}$ e $\Lambda_{x} \mid \Theta_{x}$, para todo $x \in G$. O 3-cociclo associado à família de isomorfismos de R-bimódulos $f^{\Lambda}=\left\{f_{x, y}^{\Lambda}: \Lambda_{x} \otimes_{R} \Lambda_{y} \longrightarrow 1_{x} \Lambda_{x y}, x, y \in G\right\}$ definido por

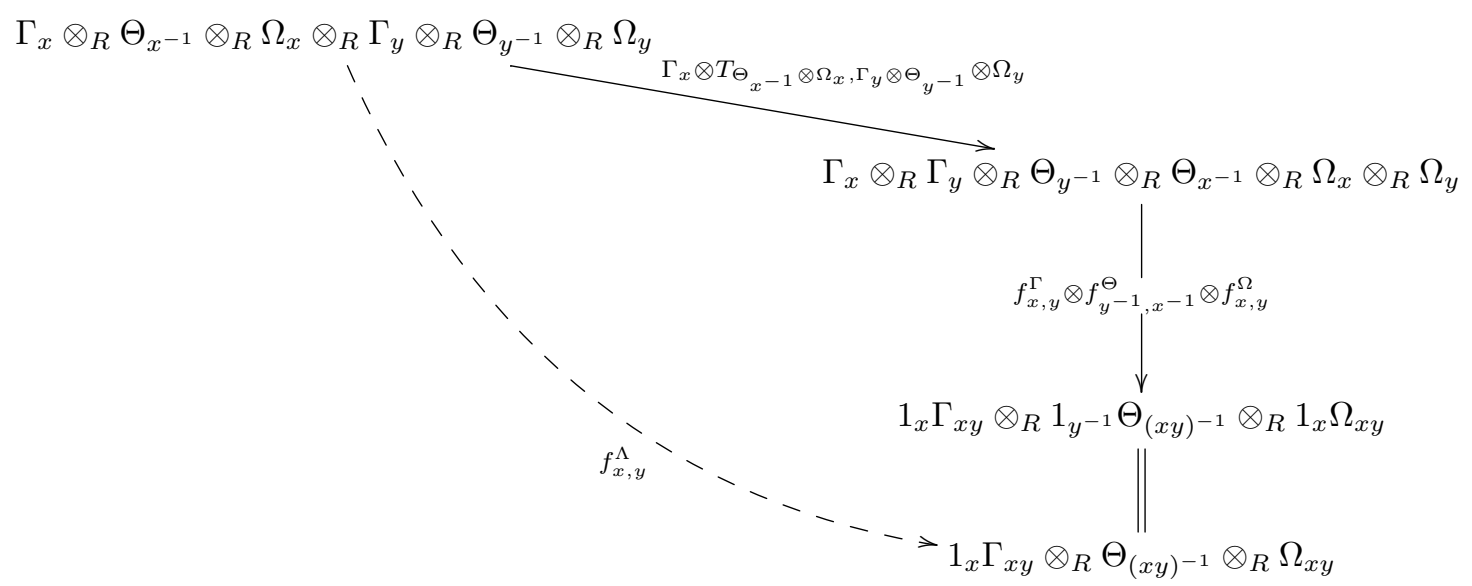

onde $T_{-,-}$é o isomorfismo da Proposição 2.3.5, é dado por $\widetilde{\beta_{-,-,-}^{\Lambda}}=\widetilde{\beta_{-,-,-}^{\Gamma} \widehat{\beta_{-,-,-}^{\Omega}}}$. Se $f^{\Gamma}$ e f f são conjuntos de fatores para $\Gamma$ e $\Omega$, respectivamente, então $f^{\Lambda}$ é um conjunto de fatores para $\Lambda$ e temos que $\Delta(\Lambda)$ é um produto cruzado generalizado parcial.

Demonstração. Claramente $\Lambda_{1}=R$. Sejam $x, y \in G$ então

$$
\begin{aligned}
\Lambda_{x^{-1}} \otimes_{R} \Lambda_{x} \otimes_{R} \Lambda_{y} & \simeq \Gamma_{x^{-1}} \otimes_{R} \Theta_{x} \otimes_{R} \Omega_{x^{-1}} \otimes_{R} R 1_{x} \otimes_{R} \Lambda_{x y} \\
& \simeq \Gamma_{x^{-1}} \otimes_{R} \Theta_{x} \otimes_{R} \Omega_{x^{-1}} \otimes_{R} \Lambda_{x y} \\
& =\Lambda_{x^{-1}} \otimes_{R} \Lambda_{x y} .
\end{aligned}
$$

Por outro lado,

$$
\begin{aligned}
\Lambda_{x} \otimes_{R} \Lambda_{y} \otimes_{R} \Lambda_{y^{-1}} & \simeq R 1_{x} \otimes_{R} \Lambda_{x y} \otimes_{R} \Lambda_{y^{-1}} \\
& \simeq R 1_{x} \otimes_{R} \Gamma_{x y} \otimes_{R} \Theta_{(x y)^{-1}} \otimes_{R} \Omega_{x y} \otimes_{R} \Gamma_{y^{-1}} \otimes_{R} \Theta_{y} \otimes_{R} \Omega_{y^{-1}} \\
& \simeq \Gamma_{x y} \otimes_{R} R 1_{y^{-1}} \otimes_{R} \Theta_{(x y)^{-1}} \otimes_{R} \Omega_{x y} \otimes_{R} \Gamma_{y^{-1}} \otimes_{R} \Theta_{y} \otimes_{R} \Omega_{y^{-1}} \\
& \simeq \Gamma_{x y} \otimes_{R} \Theta_{(x y)^{-1}} \otimes_{R} R 1_{x} \otimes_{R} \Omega_{x y} \otimes_{R} \Gamma_{y^{-1}} \otimes_{R} \Theta_{y} \otimes_{R} \Omega_{y^{-1}} \\
& \simeq \Gamma_{x y} \otimes_{R} \Theta_{(x y)^{-1}} \otimes_{R} \Omega_{x y} \otimes_{R} R 1_{y^{-1}} \otimes_{R} \Gamma_{y^{-1}} \otimes_{R} \Theta_{y} \otimes_{R} \Omega_{y^{-1}} \\
& \simeq \Gamma_{x y} \otimes_{R} \Theta_{(x y)^{-1}} \otimes_{R} \Omega_{x y} \otimes_{R} \Gamma_{y^{-1}} \otimes_{R} \Theta_{y} \otimes_{R} \Omega_{y^{-1}} \\
& =\Lambda_{x y} \otimes_{R} \Lambda_{y^{-1}} .
\end{aligned}
$$

Logo, $\Lambda$ é uma representação parcial. Observe agora que

$$
\begin{aligned}
\Lambda_{x} \otimes_{R} \Lambda_{x^{-1}} & =\Gamma_{x} \otimes_{R} \Theta_{x^{-1}} \otimes_{R} \Omega_{x} \otimes_{R} \Gamma_{x^{-1}} \otimes_{R} \Theta_{x} \otimes_{R} \Omega_{x^{-1}} \\
& \simeq \Gamma_{x} \otimes_{R} \Gamma_{x^{-1}} \otimes_{R} \Theta_{x} \otimes_{R} \Theta_{x^{-1}} \otimes_{R} \Omega_{x} \otimes_{R} \Omega_{x^{-1}} \\
& \simeq R 1_{x} \otimes_{R} R 1_{x} \otimes_{R} R 1_{x} \simeq R 1_{x} .
\end{aligned}
$$

Portanto, $\Lambda$ é unital. Como $\Gamma_{x} \mid \Theta_{x}$ e $\Omega_{x} \mid \Theta_{x}$, então $\Lambda_{x} \simeq \Gamma_{x} \otimes \Theta_{x^{-1}} \otimes \Omega_{x} \mid \Theta_{x}$, para todo $x \in G$.

Por definição temos

$$
\begin{aligned}
& \beta_{x, y, z}^{\Gamma} \circ f_{x, y z}^{\Gamma} \circ\left(\Gamma_{x} \otimes f_{y, z}^{\Gamma}\right)=f_{x y, z}^{\Gamma} \otimes\left(f_{x, y}^{\Gamma} \otimes \Gamma_{z}\right), \\
& \beta_{x, y, z}^{\Omega} \circ f_{x, y z}^{\Omega} \circ\left(\Omega_{x} \otimes f_{y, z}^{\Omega}\right)=f_{x y, z}^{\Omega} \otimes\left(f_{x, y}^{\Omega} \otimes \Omega_{z}\right) .
\end{aligned}
$$

Vamos mostrar que $\widetilde{\beta_{x, y, z}^{\Gamma}} \widetilde{\beta_{x, y, z}^{\Omega}} f_{x, y z}^{\Lambda} \circ\left(\Lambda_{x} \otimes f_{y, z}^{\Lambda}\right)=f_{x y, z}^{\Lambda} \circ\left(f_{x, y}^{\Lambda} \otimes \Lambda_{z}\right)$, para todos $x, y, z \in G$. 
A composta $f_{x y, z}^{\Lambda} \circ\left(f_{x, y}^{\Lambda} \otimes \Lambda_{z}\right)$ é dada por:

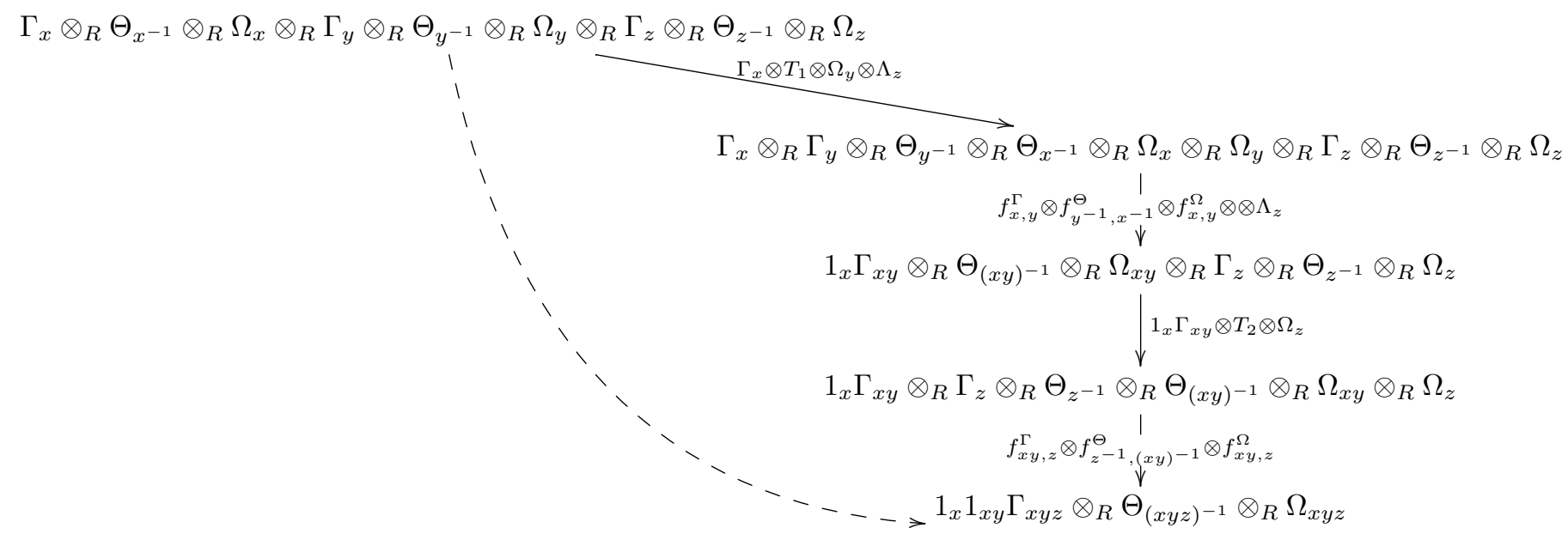

onde $T_{1}$ e $T_{2}$ são os isomorfismos

$$
\begin{gathered}
T_{1}: \Theta_{x^{-1}} \otimes_{R} \Omega_{x} \otimes_{R} \Gamma_{y} \otimes_{R} \Theta_{y^{-1}} \longrightarrow \Gamma_{y} \otimes_{R} \Theta_{y^{-1}} \otimes_{R} \Theta_{x^{-1}} \otimes_{R} \Omega_{x}, \\
T_{2}: \Theta_{(x y)^{-1}} \otimes_{R} \Omega_{x y} \otimes_{R} \Gamma_{z} \otimes_{R} \Theta_{z^{-1}} \longrightarrow \Gamma_{z} \otimes_{R} \Theta_{z^{-1}} \otimes_{R} \Theta_{(x y)^{-1}} \otimes_{R} \Omega_{x y} .
\end{gathered}
$$

Por outro lado, $f_{x, y z}^{\Lambda} \circ\left(\Lambda_{x} \otimes f_{y, z}^{\Lambda}\right)$ é dado por $\Gamma_{x} \otimes_{R} \Theta_{x^{-1}} \otimes_{R} \Omega_{x} \otimes_{R} \Gamma_{y} \otimes_{R} \Theta_{y^{-1}} \otimes_{R} \Omega_{y} \otimes_{R} \Gamma_{z} \otimes_{R} \Theta_{z^{-1}} \otimes_{R} \Omega_{z}$

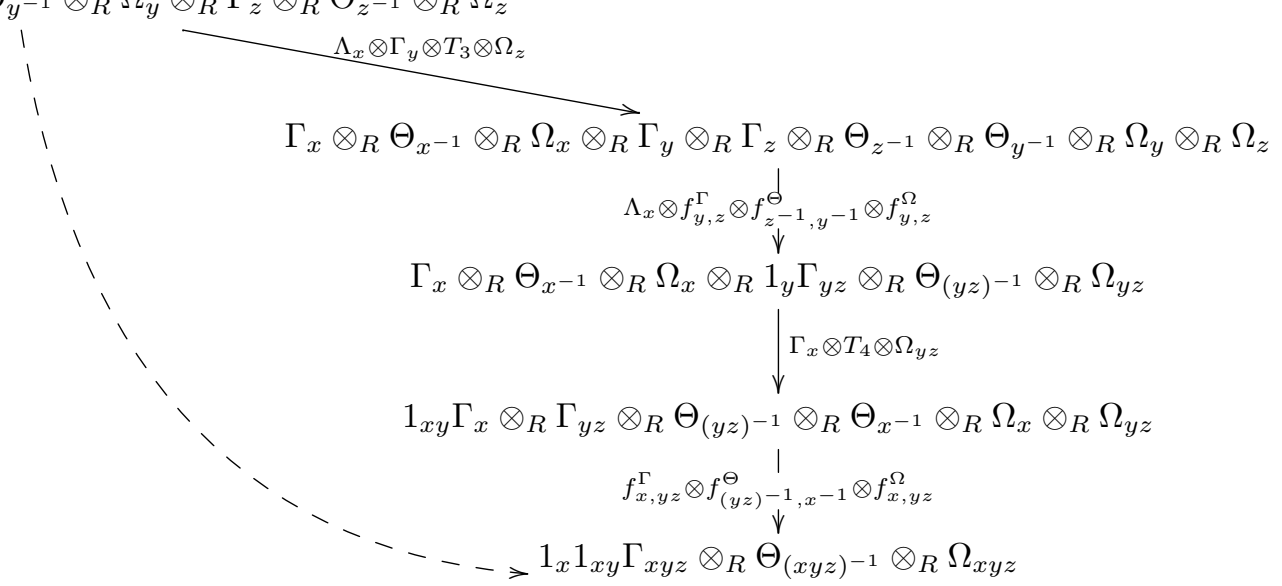

onde $T_{3}$ e $T_{4}$ são os isomorfismos

$$
\begin{gathered}
T_{3}: \Theta_{y^{-1}} \otimes_{R} \Omega_{y} \otimes_{R} \Gamma_{z} \otimes_{R} \Theta_{z^{-1}} \longrightarrow \Gamma_{z} \otimes_{R} \Theta_{z^{-1}} \otimes_{R} \Theta_{y^{-1}} \otimes_{R} \Omega_{y}, \\
T_{4}: \Theta_{x^{-1}} \otimes_{R} \Omega_{x} \otimes_{R} \Gamma_{y z} \otimes_{R} \Theta_{(y z)^{-1}} \longrightarrow \Gamma_{y z} \otimes_{R} \Theta_{(y z)^{-1}} \otimes_{R} \Theta_{x^{-1}} \otimes_{R} \Omega_{x} .
\end{gathered}
$$

Vamos construir os isomorfismos $T_{i}$, com $i=1,2,3,4$. Para isso, considere as aplicações $R$-bilinear $\varphi_{i}: \Theta_{x^{-1}} \otimes_{R} \Omega_{x} \rightarrow R$ e $\psi_{i}: R \rightarrow \Theta_{x^{-1}} \otimes_{R} \Omega_{x}, \operatorname{com} i=1,2, \ldots, n$, tais que $\sum_{i=1}^{n} \psi_{i} \varphi_{i}=I d_{\Theta_{x^{-1}} \otimes \Omega_{x}}$ e $\varphi_{j}^{\prime}: \Gamma_{z} \otimes_{R} \Theta_{z^{-1}} \rightarrow R$ e $\psi_{j}^{\prime}: R \rightarrow \Gamma_{z} \otimes_{R} \Theta_{z^{-1}}, \operatorname{com} j=1,2, \ldots, m$, tais que $\sum_{j=1}^{m} \psi_{j}^{\prime} \varphi_{j}^{\prime}=I d_{\Gamma_{z} \otimes \Theta_{z^{-1}}}$. Denote $\psi_{i}(1)=\sum_{l} u_{x^{-1}}^{i, l} \otimes \omega_{x}^{i, l} \quad$ e $\quad \psi_{j}^{\prime}(1)=\sum_{k} \widetilde{v}_{z}^{j, k} \otimes \widetilde{u}_{z^{-1}}^{j, k}$. 
Pelo Lema 2.3.5, temos:

$$
\begin{aligned}
& T_{1}: \quad \Theta_{x^{-1}} \otimes_{R} \Omega_{x} \otimes_{R} \Gamma_{y} \otimes_{R} \Theta_{y^{-1}} \rightarrow \Gamma_{y} \otimes_{R} \Theta_{y^{-1}} \otimes_{R} \Theta_{x^{-1}} \otimes_{R} \Omega_{x} \\
& u_{x^{-1}} \otimes \omega_{x} \otimes v_{y} \otimes u_{y^{-1}} \mapsto \sum_{i, l} \varphi_{i}\left(u_{x^{-1}} \otimes \omega_{x}\right) v_{y} \otimes u_{y^{-1}} \otimes u_{x^{-1}}^{i, l} \otimes \omega_{x}^{i, l} \\
& T_{2}: \quad \Theta_{(x y)^{-1}} \otimes_{R} \Omega_{x y} \otimes_{R} \Gamma_{z} \otimes_{R} \Theta_{z^{-1}} \rightarrow \Gamma_{z} \otimes_{R} \Theta_{z^{-1}} \otimes_{R} \Theta_{(x y)^{-1}} \otimes_{R} \Omega_{x y} \\
& u_{(x y)^{-1}} \otimes \omega_{x y} \otimes v_{z} \otimes u_{z^{-1}} \quad \mapsto \sum_{j, k} \widetilde{v}_{z}^{j, k} \otimes \widetilde{u}_{z^{-1}}^{j, k} \otimes u_{(x y)^{-1}} \otimes \omega_{x y} \varphi_{j}^{\prime}\left(v_{z} \otimes u_{z^{-1}}\right), \\
& T_{3}: \quad \Theta_{y^{-1}} \otimes_{R} \Omega_{y} \otimes_{R} \Gamma_{z} \otimes_{R} \Theta_{z^{-1}} \rightarrow \Gamma_{z} \otimes_{R} \Theta_{z^{-1}} \otimes_{R} \Theta_{y^{-1}} \otimes_{R} \Omega_{y} \\
& u_{y^{-1}} \otimes \omega_{y} \otimes v_{z} \otimes u_{z^{-1}} \quad \mapsto \sum_{j, k} \widetilde{v}_{z}^{j, k} \otimes \widetilde{u}_{z^{-1}}^{j, k} \otimes u_{y^{-1}} \otimes \omega_{y} \varphi_{j}^{\prime}\left(v_{z} \otimes u_{z^{-1}}\right), \\
& T_{4}: \quad \Theta_{x^{-1}} \otimes_{R} \Omega_{x} \otimes_{R} \Gamma_{y z} \otimes_{R} \Theta_{(y z)^{-1}} \rightarrow \Gamma_{y z} \otimes_{R} \Theta_{(y z)^{-1}} \otimes_{R} \Theta_{x^{-1}} \otimes_{R} \Omega_{x} \\
& u_{x^{-1}} \otimes \omega_{x} \otimes v_{y z} \otimes u_{(y z)^{-1}} \mapsto \sum_{i, l} \varphi_{i}\left(u_{x^{-1}} \otimes \omega_{x}\right) v_{y z} \otimes u_{(y z)^{-1}} \otimes u_{x^{-1}}^{i, l} \otimes \omega_{x}^{i, l} .
\end{aligned}
$$

Então, $f_{x, y z}^{\Lambda} \circ\left(\Lambda_{x} \otimes f_{y, z}^{\Lambda}\right)$ é dado por:

$v_{x} \otimes u_{x^{-1}} \otimes \omega_{x} \otimes v_{y} \otimes u_{y^{-1}} \otimes \omega_{y} \otimes v_{z} \otimes u_{z^{-1}} \otimes \omega_{z}$

$\stackrel{T_{3}}{\mapsto} \sum_{j, k} v_{x} \otimes u_{x^{-1}} \otimes \omega_{x} \otimes v_{y} \otimes \widetilde{v}_{z}^{j, k} \otimes \widetilde{u}_{z^{-1}}^{j, k} \otimes u_{y^{-1}} \otimes \omega_{y} \varphi_{j}^{\prime}\left(v_{z} \otimes u_{z^{-1}}\right) \otimes \omega_{z}$

$\mapsto \sum_{j, k} v_{x} \otimes u_{x^{-1}} \otimes \omega_{x} \otimes f_{y, z}^{\Gamma}\left(v_{y} \otimes \widetilde{v}_{z}^{j, k}\right) \otimes\left(\widetilde{u}_{z^{-1}}^{j, k} \stackrel{\ominus}{\circ} u_{y^{-1}}\right) \otimes f_{y, z}^{\Omega}\left(\omega_{y} \varphi_{j}^{\prime}\left(v_{z} \otimes u_{z^{-1}}\right) \otimes \omega_{z}\right)$

$\stackrel{T_{4}}{\mapsto} \sum_{i, j, k, l} v_{x} \otimes \varphi_{i}\left(u_{x^{-1}} \otimes \omega_{x}\right) f_{y, z}^{\Gamma}\left(v_{y} \otimes \widetilde{v}_{z}^{j, k}\right) \otimes\left(\widetilde{u}_{z^{-1}}^{j, k} \stackrel{\ominus}{\circ} u_{y^{-1}}\right) \otimes u_{x^{-1}}^{i, l} \otimes \omega_{x}^{i, l} \otimes f_{y, z}^{\Omega}\left(\omega_{y} \varphi_{j}^{\prime}\left(v_{z} \otimes u_{z^{-1}}\right) \otimes \omega_{z}\right)$

$\mapsto \sum_{i, j, k, l} f_{x, y z}^{\Gamma}\left(v_{x} \otimes \varphi_{i}\left(u_{x^{-1}} \otimes \omega_{x}\right) f_{y, z}^{\Gamma}\left(v_{y} \otimes \widetilde{v}_{z}^{j, k}\right)\right) \otimes\left(\left(\widetilde{u}_{z^{-1}}^{j, k} \stackrel{\ominus}{\circ} u_{y^{-1}}\right) \stackrel{\ominus}{\circ} u_{x^{-1}}^{i, l}\right)$

$\otimes f_{x, y z}^{\Omega}\left(\omega_{x}^{i, l} \otimes f_{y, z}^{\Omega}\left(\omega_{y} \varphi_{j}^{\prime}\left(v_{z} \otimes u_{z^{-1}}\right) \otimes \omega_{z}\right)\right)$

Por outro lado, $f_{x y, z}^{\Lambda} \circ\left(f_{x, y}^{\Lambda} \otimes \Lambda_{z}\right)$ é dado por:

$v_{x} \otimes u_{x^{-1}} \otimes \omega_{x} \otimes v_{y} \otimes u_{y^{-1}} \otimes \omega_{y} \otimes v_{z} \otimes u_{z^{-1}} \otimes \omega_{z}$

$\stackrel{T_{1}}{\mapsto} \sum_{i, l} v_{x} \otimes \varphi_{i}\left(u_{x^{-1}} \otimes \omega_{x}\right) v_{y} \otimes u_{y^{-1}} \otimes u_{x^{-1}}^{i, l} \otimes \omega_{x}^{i, l} \otimes \omega_{y} \otimes v_{z} \otimes u_{z^{-1}} \otimes \omega_{z}$

$\mapsto \sum_{i, l} f_{x, y}^{\Gamma}\left(v_{x} \otimes \varphi_{i}\left(u_{x^{-1}} \otimes \omega_{x}\right) v_{y}\right) \otimes\left(u_{y^{-1}} \stackrel{\ominus}{\circ} u_{x^{-1}}^{i, l}\right) \otimes f_{x, y}^{\Omega}\left(\omega_{x}^{i, l} \otimes \omega_{y}\right) \otimes v_{z} \otimes u_{z^{-1}} \otimes \omega_{z}$

$\stackrel{T_{2}}{\mapsto} \sum_{i, j, k, l} f_{x, y}^{\Gamma}\left(v_{x} \otimes \varphi_{i}\left(u_{x^{-1}} \otimes \omega_{x}\right) v_{y}\right) \otimes \widetilde{v}_{z}^{j, k} \otimes \widetilde{u}_{z^{-1}}^{j, k} \otimes\left(u_{y^{-1}} \stackrel{\ominus}{\circ} u_{x^{-1}}^{i, l}\right) \otimes f_{x, y}^{\Omega}\left(\omega_{x}^{i, l} \otimes \omega_{y}\right) \varphi_{j}^{\prime}\left(v_{z} \otimes u_{z^{-1}}\right) \otimes \omega_{z}$

$\mapsto \sum_{i, j, k, l} f_{x y, z}^{\Gamma}\left(f_{x, y}^{\Gamma}\left(v_{x} \otimes \varphi_{i}\left(u_{x^{-1}} \otimes \omega_{x}\right) v_{y}\right) \otimes \widetilde{v}_{z}^{j, k}\right) \otimes\left(\widetilde{u}_{z^{-1}}^{j, k} \stackrel{\ominus}{\circ}\left(u_{y^{-1}} \stackrel{\ominus}{\circ} u_{x^{-1}}^{i, l}\right)\right)$

$\otimes f_{x y, z}^{\Omega}\left(f_{x, y}^{\Omega}\left(\omega_{x}^{i, l} \otimes \omega_{y} \varphi_{j}^{\prime}\left(v_{z} \otimes u_{z^{-1}}\right)\right) \otimes \omega_{z}\right)$ 


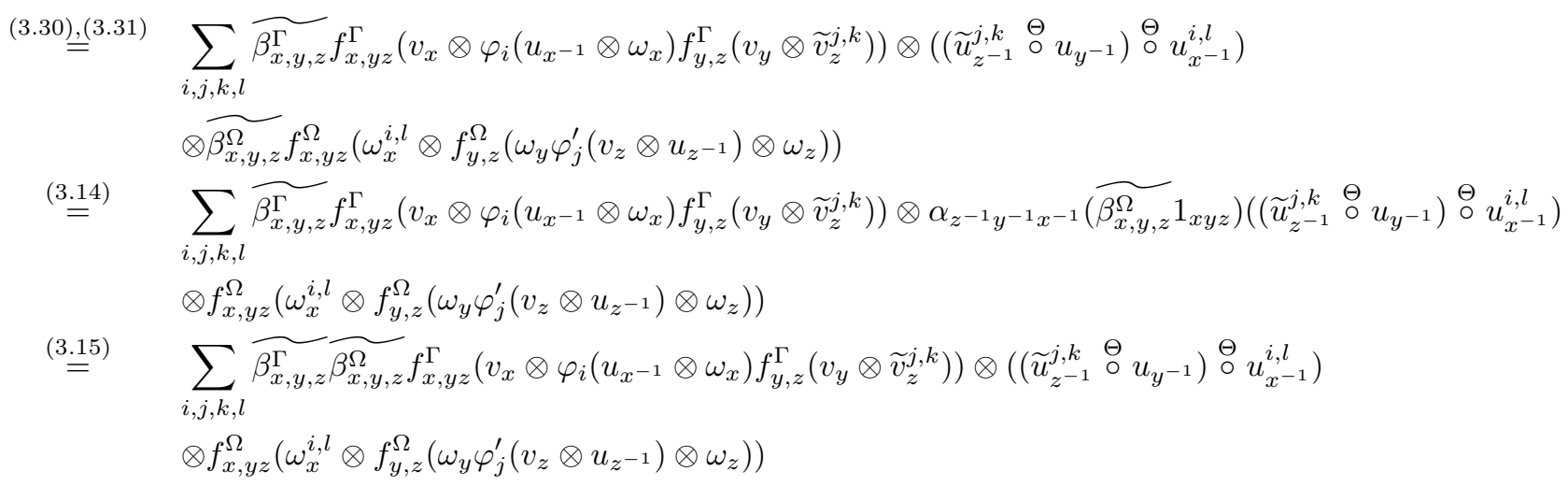

Então,

$$
\widetilde{\beta_{x, y, z}^{\Gamma}} \widetilde{\beta_{x, y, z}^{\Omega}} f_{x, y z}^{\Lambda} \circ\left(\Lambda_{x} \otimes f_{y, z}^{\Lambda}\right)=f_{x y, z}^{\Lambda} \circ\left(f_{x, y}^{\Lambda} \otimes \Lambda_{z}\right) \text {, para todo } x, y, z \in G .
$$

Portanto, $\widetilde{\beta_{-,-,-}^{\Lambda}}=\widetilde{\beta_{-,-,-}^{\Gamma}} \widetilde{\beta_{-,-,-}^{\Omega}}$

Se $f^{\Gamma}$ e $f^{\Omega}$ são conjuntos de fatores, então $\widetilde{\beta_{-,-,-}^{\Gamma}}$ e $\widetilde{\beta_{-,-,-}^{\Omega}}$ são triviais. Assim, $\widetilde{\beta_{-,-,-}^{\Lambda}}$ também é trivial. Logo, $f^{\Lambda}$ é um conjunto de fatores para $\Lambda$. Portanto, $\Delta(\Lambda)$ é um produto cruzado generalizado parcial.

Definição 3.3.9. Sejam $\Delta(\Theta)$ e $\Delta(\Gamma)$ produtos cruzados generalizados parciais com isomorfismos de $R$-bimódulos e anéis $i: R \longrightarrow \Theta_{1}$ e $j: R \longrightarrow \Gamma_{1}$ como na Observação 3.3.4. Um morfismo de produtos cruzados generalizados parciais $F: \Delta(\Theta) \longrightarrow \Delta(\Gamma)$ é um conjunto de morfismos $\left\{F_{x}: \Theta_{x} \longrightarrow \Gamma_{x}, x \in G\right\}$ tal que $F_{1} \circ i=j$ e o diagrama

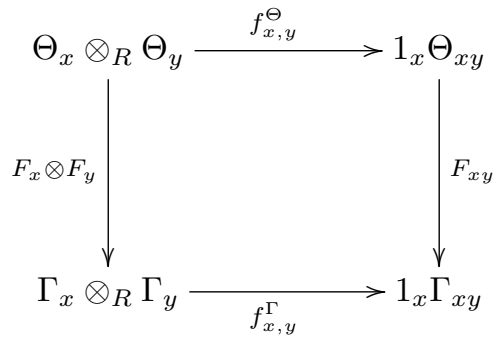

seja comutativo. Um morfismo de produtos cruzados generalizados parciais $F$ é dito um isomorfismo se cada $F_{x}: \Theta_{x} \longrightarrow \Gamma_{x}$ é um isomorfismo de R-bimódulos.

É importante observar que se temos uma família de isomorfismos $F=\left\{F_{x}: \Theta_{x} \longrightarrow \Gamma_{x}, x \in G\right\}$ que satisfazem o diagrama comutativo (3.32), então $F$ é um isomorfismo de produtos cruzados generalizados parciais, ou seja, $F_{1} \circ i=j$ será satisfeita automaticamente. De fato, a comutatividade do diagrama (3.32) e dos diagramas na Observação 3.3.4 implicam que o diagrama abaixo é comutativo

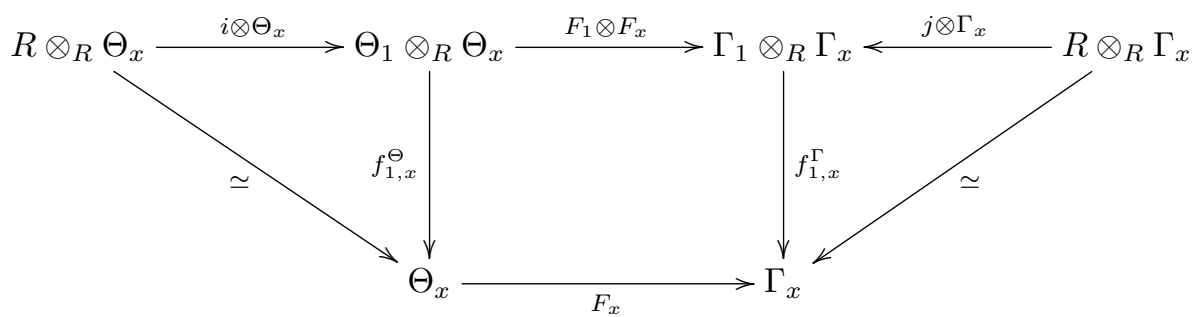


Seja $v_{x} \in \Gamma_{x}$, então existe $u_{x} \in \Theta_{x}$ tal que $F_{x}\left(u_{x}\right)=v_{x}$. Pela comutatividade do diagrama temos $\left(F_{1}(i(1)) \stackrel{\Gamma}{\circ} F_{x}\left(u_{x}\right)\right)=F_{x}\left(u_{x}\right)$, ou seja,

$$
\left(F_{1}(i(1)) \stackrel{\Gamma}{\circ} v_{x}\right)=v_{x}, \text { para todo } v_{x} \in \Gamma_{x} .
$$

Por outro lado, a comutatividade do diagrama

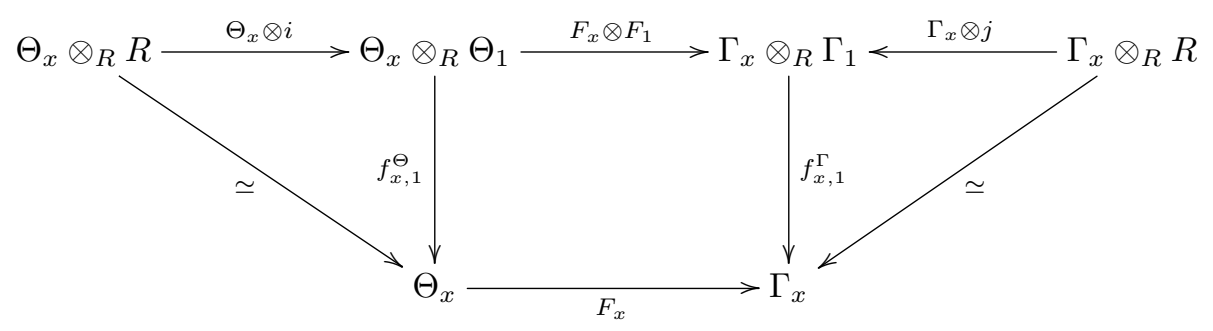

implica que $\left(v_{x} \circ F_{1}(i(1))\right)=v_{x}$, para todo $v_{x} \in \Gamma_{x}$. Logo, $F(i(1))=j(1)$ é a unidade de $\Delta(\Gamma)$. Portanto, $F \circ i=j$.

Observação 3.3.10. Seja $f^{\Theta}=\left\{f_{x, y}^{\Theta}: \Theta_{x} \otimes_{R} \Theta_{y} \longrightarrow 1_{x} \Theta_{x y}, x, y \in G\right\}$ um conjunto de fatores para $\Theta$. Para cada $x \in G$ temos um isomorfismo de R-bimódulos $f_{x, x^{-1}}^{\Theta}: \Theta_{x} \otimes \Theta_{x^{-1}} \longrightarrow R 1_{x}$, cujo inverso é dada

$$
\begin{aligned}
\left(f_{x, x^{-1}}^{\Theta}\right)^{-1}: \quad R 1_{x} & \longrightarrow \Theta_{x} \otimes_{R} \Theta_{x^{-1}} \\
r 1_{x} & \longrightarrow \sum_{(x)} r \omega_{x} \otimes \omega_{x^{-1}}
\end{aligned}
$$

onde $\sum_{(x)}\left(\omega_{x} \stackrel{\ominus}{\circ} \omega_{x^{-1}}\right)=1_{x}$. Restringindo o isomorfismo $f_{x y,(x y)^{-1}}^{\Theta}$ a $1_{x} \Theta_{x y} \otimes_{R} \Theta_{(x y)^{-1}}$ temos um isomorfismo de R-bimódulos $1_{x} f_{x y,(x y)^{-1}}^{\Theta}: 1_{x} \Theta_{x y} \otimes_{R} \Theta_{(x y)^{-1}} \longrightarrow R 1_{x} 1_{x y}$. Sejam

$$
1_{x}=\sum_{(x)}\left(\omega_{x} \stackrel{\ominus}{\circ} \omega_{x^{-1}}\right) \text { e } 1_{y}=\sum_{(y)}\left(\omega_{y} \stackrel{\ominus}{\circ} \omega_{y^{-1}}\right) .
$$

Pela associatividade, temos

$$
\begin{aligned}
\sum_{(x),(y)}\left(\left(\omega_{x} \stackrel{\ominus}{\circ} \omega_{y}\right) \stackrel{\ominus}{\circ}\left(\omega_{y^{-1}} \stackrel{\ominus}{\circ} \omega_{x^{-1}}\right)\right) & =\sum_{(x),(y)}\left(\omega_{x} \stackrel{\ominus}{\circ}\left(\omega_{y} \stackrel{\ominus}{\circ} \omega_{y^{-1}}\right) \stackrel{\ominus}{\circ} \omega_{x^{-1}}\right) \\
& =\sum_{(x)}\left(\omega_{x} 1_{y} \stackrel{\ominus}{\circ} \omega_{x^{-1}}\right)=\sum_{(x)} 1_{x y}\left(\omega_{x} \stackrel{\ominus}{\circ} \omega_{x^{-1}}\right) \\
& =1_{x y} 1_{x} .
\end{aligned}
$$

Então,

$$
\begin{aligned}
& \left(1_{x} f_{x y, y^{-1} x^{-1}}^{\Theta}\right)^{-1}: \quad R 1_{x} 1_{x y} \quad \longrightarrow \quad 1_{x} \Theta_{x y} \otimes_{R} \Theta_{(x y)^{-1}} \\
& r 1_{x} 1_{1_{x y}} \longmapsto \sum_{(x),(y)} r\left(\left(\omega_{x} \stackrel{\ominus}{\circ} \omega_{y}\right) \otimes\left(\omega_{y^{-1}} \stackrel{\ominus}{\circ} \omega_{x^{-1}}\right)\right) .
\end{aligned}
$$


De fato, seja $r \in R 1_{x} 1_{x y}$ e $\omega_{x y} \in \Theta_{x y}, \omega_{(x y)^{-1}} \in \Theta_{(x y)^{-1}} \operatorname{com} \sum_{(x y)} \omega_{x y} \stackrel{\ominus}{\circ} \omega_{(x y)^{-1}}=1_{x y}$. Então

$$
\begin{aligned}
& \sum_{(x y)} r \omega_{x y} \otimes \omega_{(x y)^{-1}}=\sum_{(x y)} r 1_{x} 1_{x y} \omega_{x y} \otimes \omega_{(x y)^{-1}} \\
& =\sum_{(x),(y),(x y)} r\left(\left(\omega_{x} \stackrel{\ominus}{\circ} \omega_{y}\right) \stackrel{\ominus}{\circ}\left(\omega_{y^{-1}} \stackrel{\ominus}{\circ} \omega_{x^{-1}}\right)\right) \omega_{x y} \otimes \omega_{(x y)^{-1}} \\
& =\sum_{(x),(y),(x y)} r\left(\left(\omega_{x} \stackrel{\ominus}{\circ} \omega_{y}\right) \stackrel{\ominus}{\circ}\left(\omega_{y^{-1}} \stackrel{\ominus}{\circ} \omega_{x^{-1}}\right) \stackrel{\ominus}{\circ} \omega_{x y}\right) \otimes \omega_{(x y)^{-1}} \\
& =\sum_{(x),(y),(x y)} r\left(\omega_{x} \stackrel{\ominus}{\circ} \omega_{y}\right) \underbrace{\left(\left(\omega_{y^{-1}} \stackrel{\ominus}{\circ} \omega_{x^{-1}}\right) \stackrel{\ominus}{\circ} \omega_{x y}\right)}_{\in R} \otimes \omega_{(x y)^{-1}} \\
& =\sum_{(x),(y),(x y)} r\left(\omega_{x} \stackrel{\ominus}{\circ} \omega_{y}\right) \otimes\left(\left(\omega_{y^{-1}} \stackrel{\ominus}{\circ} \omega_{x^{-1}}\right) \stackrel{\ominus}{\circ} \omega_{x y}\right) \omega_{(x y)^{-1}} \\
& =\sum_{(x),(y),(x y)} r\left(\omega_{x} \stackrel{\ominus}{\circ} \omega_{y}\right) \otimes\left(\omega_{y^{-1}} \stackrel{\ominus}{\circ} \omega_{x^{-1}}\right)\left(\omega_{x y} \stackrel{\ominus}{\circ} \omega_{(x y)^{-1}}\right) \\
& =\sum_{(x),(y)} r\left(\omega_{x} \stackrel{\ominus}{\circ} \omega_{y}\right) \otimes\left(\omega_{y^{-1}} \stackrel{\ominus}{\circ} \omega_{x^{-1}}\right) 1_{x y} \\
& =\sum_{(x),(y)} r\left(\omega_{x} \stackrel{\ominus}{\circ} \omega_{y}\right) \otimes\left(\omega_{y^{-1}} \stackrel{\ominus}{\circ} 1_{y} \omega_{x^{-1}}\right) \\
& =\sum_{(x),(y)} r\left(\omega_{x} \stackrel{\ominus}{\circ} \omega_{y}\right) \otimes\left(\omega_{y^{-1}} \stackrel{\ominus}{\circ} \omega_{x^{-1}}\right) .
\end{aligned}
$$

\subsubsection{Representações parciais unitais em $\mathcal{S}_{R}(S)$.}

Nesta seção vamos relacionar um produto cruzado parcial generalizado com uma extensão de anéis com mesma unidade. Já vimos na Seção 3.3 que dada uma representação parcial unital $\Theta: G \longrightarrow \operatorname{PicS}(R)$ de tal forma que tenhamos um produto cruzado generalizado parcial $\Delta(\Theta)$, então $R \subseteq \Delta(\Theta)$ é uma extensão de anéis com mesma unidade. Vamos ver como obter um produto cruzado generalizado a partir de uma extensão de anéis $R \subseteq S$ e uma representação parcial.

Seja $R \subseteq S$ uma extensão de anéis com mesma unidade e denote $\mathcal{S}_{R}(S)$ o conjunto dos $R$-subbimódulos de $S$. Dados $M, N \in \mathcal{S}_{R}(S)$ definindo o produto por

$$
M N=\left\{\sum_{i=1}^{k} m_{i} n_{i} ; m_{i} \in M, n_{i} \in N\right\} .
$$

Então, $\mathcal{S}_{R}(S)$ é monoide onde o elemento neutro é anel $R$.

Uma representação parcial

$$
\begin{aligned}
\Theta: G & \longrightarrow \mathcal{S}_{R}(S) \\
x & \longmapsto \Theta_{x}
\end{aligned}
$$

é dita unital se $\varepsilon_{x}=\Theta_{x} \Theta_{x^{-1}}=R 1_{x}$, onde $1_{x}$ é um idempotente central em $R$, para todo $x \in G$. De, $\Theta_{x} \Theta_{x^{-1}} \Theta_{x}=\Theta_{x}$, para todo $x \in G$, temos que $R 1_{x} \Theta_{x}=\Theta_{x}$ e $\Theta_{x} R 1_{x^{-1}}=\Theta_{x}$, logo $1_{x} u_{x}=u_{x}$ e $u_{x} 1_{x^{-1}}=u_{x}$, para todo $u_{x} \in \Theta_{x}$ e $x \in G$.

Proposição 3.3.11. Seja $\Theta: G \longrightarrow \mathcal{S}_{R}(S)$ uma representação parcial unital. Então, 
(i) $\left[\Theta_{x}\right] \in \mathbf{P i c} \mathbf{S}(R)$.

(ii) Se M é um S-bimódulo, então

$$
\begin{aligned}
m_{l}: \Theta_{x} \otimes_{R} M & \longrightarrow \Theta_{x} M \\
u_{x} \otimes m & \longmapsto u_{x} m
\end{aligned} \quad \text { e } \begin{aligned}
m_{r}: M \otimes_{R} \Theta_{x} & \longrightarrow M \Theta_{x} \\
m \otimes u_{x} & \longmapsto m u_{x}
\end{aligned}
$$

são isomorfismos de $R$-S-bimódulos e $S$-R-bimódulos, respectivamente.

(iii) Sejam $M$ um S-bimódulo e $N$ um R-subbimódulo de $M$. Então, temos os seguintes isomorfismos de R-bimódulos:

$$
\begin{array}{cl}
\Theta_{x} \otimes_{R} N & \longrightarrow \Theta_{x} N \\
u_{x} \otimes n & \longmapsto u_{x} n
\end{array} \quad \text { e } \begin{aligned}
N \otimes_{R} \Theta_{x} & \longrightarrow N \Theta_{x} \\
n \otimes u_{x^{-1}} & \longmapsto n u_{x^{-1}}
\end{aligned}
$$

Demonstração. (i) Sejam $\omega_{x}^{i} \in \Theta_{x}$ e $\omega_{x-1}^{i} \in \Theta_{x^{-1}}$ tais que $\sum_{i=1}^{m} \omega_{x}^{i} \omega_{x^{-1}}^{i}=1_{x}$. Defina,

$$
\begin{aligned}
f_{i}: \Theta_{x} & \longrightarrow R \\
u_{x} & \longmapsto \omega_{x^{-1}}^{i} u_{x}
\end{aligned} .
$$

Então, $f_{i}$ é $R$-linear à direita e temos

$$
\sum_{i=1}^{n} \omega_{x}^{i} f_{i}\left(u_{x}\right)=\sum_{i=1}^{n} \omega_{x}^{i} \omega_{x-1}^{i} u_{x}=1_{x} u_{x}=u_{x}
$$

para todo $u_{x} \in \Theta_{x}$. Logo, $\left\{\omega_{x}^{i}, f_{i}\right\}$ é base dual de $\Theta_{x}$ como $R$-módulo à direita.

Analogamente, sejam $\bar{\omega}_{x}^{j} \in \Theta_{x}$ e $\bar{\omega}_{x^{-1}}^{j} \in \Theta_{x^{-1}}, \operatorname{com} j=1,2, \ldots, m$, tais que $\sum_{j=1}^{m} \bar{\omega}_{x^{-1}}^{j} \bar{\omega}_{x}^{j}=1_{x^{-1}}$. Defina

$$
\begin{aligned}
g_{j}: \Theta_{x} & \longrightarrow R \\
u_{x} & \longrightarrow u_{x} \bar{\omega}_{x^{-1}}^{j} .
\end{aligned}
$$

Então, $g_{j}$ é $R$-linear à esquerda e temos

$$
\sum_{j=1}^{m} g_{j}\left(u_{x}\right) \bar{\omega}_{x}^{j}=\sum_{j=1}^{m} u_{x} \bar{\omega}_{x^{-1}}^{j} \bar{\omega}_{x}^{j}=u_{x} 1_{x^{-1}}=u_{x}
$$

para todo $u_{x} \in \Theta_{x}$. Logo, $\left\{\bar{\omega}_{x}^{j}, g_{j}\right\}$ é base dual para $\Theta_{x}$ como $R$-módulo à esquerda.

Seja $\varphi: \Theta_{x} \longrightarrow \Theta_{x} R$-linear à direita. Então, $\widetilde{\varphi}=\sum_{i=1}^{n} \varphi\left(\omega_{x}^{i}\right) \omega_{x^{-1}}^{i} \in R 1_{x}$ e temos

$$
\widetilde{\varphi} u_{x}=\sum_{i=1}^{n} \varphi\left(\omega_{x}^{i}\right) \omega_{x^{-1}}^{i} u_{x}=\sum_{i=1}^{n} \varphi\left(\omega_{x}^{i} \omega_{x^{-1}}^{i} u_{x}\right)=\varphi\left(1_{x} u_{x}\right)=\varphi\left(u_{x}\right)
$$

para todo $u_{x} \in \Theta_{x}$. Então, a aplicação $R \longrightarrow \operatorname{End}\left(\Theta_{x R}\right)$ definida por $r \longmapsto\left(u_{x} \mapsto r u_{x}\right)$ é sobrejetora.

Analogamente, se $\psi: \Theta_{x} \longrightarrow \Theta_{x}$ é $R$-linear à esquerda, então $\widetilde{\psi}=\sum_{j=1}^{m} \bar{\omega}_{x^{-1}}^{j} \psi\left(\bar{\omega}_{x}^{j}\right) \in R 1_{x^{-1}}$ e temos

$$
u_{x} \widetilde{\psi}=\sum_{j=1}^{m} u_{x} \bar{\omega}_{x-1}^{j} \psi\left(\bar{\omega}_{x}^{j}\right)=\sum_{j=1}^{m} \psi\left(u_{x} \bar{\omega}_{x-1}^{j} \bar{\omega}_{x}^{j}\right)=\psi\left(u_{x} 1_{x^{-1}}\right)=\psi\left(u_{x}\right),
$$


para todo $u_{x} \in \Theta_{x}$. Logo, a aplicação $R \longrightarrow \operatorname{End}\left({ }_{R} \Theta_{x}\right)$ definida por $r \longmapsto\left(u_{x} \mapsto u_{x} r\right)$ é sobrejetora. Portanto, $\left[\Theta_{x}\right] \in \mathbf{P i c S}(R)$.

(ii) Claramente $m_{l}$ é bem definida e $R$-S-bilinear. Sua inversa é dada por

$$
\begin{aligned}
m_{l}^{-1}: \Theta_{x} M & \longrightarrow \Theta_{x} \otimes_{R} M \\
m & \longmapsto \sum_{(x)} \omega_{x} \otimes \omega_{x^{-1}} m,
\end{aligned}
$$

onde $1_{x}=\sum_{(x)} \omega_{x} \omega_{x^{-1}}$, com $\omega_{x} \in \Theta_{x}$ e $\omega_{x^{-1}} \in \Theta_{x^{-1}}$. De fato, vejamos que $m_{l}^{-1}$ está bem definida. Seja $1_{x}=\sum_{\widetilde{x}} \widetilde{\omega}_{x} \widetilde{\omega}_{x^{-1}}$ outra decomposição de $1_{x}$. Então,

$$
\begin{aligned}
m_{l}^{-1}(m) & =\sum_{(x)} \omega_{x} \otimes \omega_{x^{-1}} m=\sum_{(x)} \omega_{x} \otimes \omega_{x^{-1}} 1_{x} m=\sum_{(x),(\widetilde{x})} \omega_{x} \otimes \omega_{x^{-1}} \widetilde{\omega}_{x} \widetilde{\omega}_{x^{-1}} m \\
& =\sum_{(x),(\widetilde{x})} \omega_{x} \omega_{x^{-1}} \widetilde{\omega}_{x} \otimes \widetilde{\omega}_{x^{-1}} m=\sum_{(\widetilde{x})} 1_{x} \widetilde{\omega}_{x} \otimes \widetilde{\omega}_{x^{-1}} m=\sum_{(\widetilde{x})} \widetilde{\omega}_{x} \otimes \widetilde{\omega}_{x^{-1}} m .
\end{aligned}
$$

Observe que $\Theta_{x} M=1_{x} M=\left\{m \in M, 1_{x} m=m\right\}$. De fato, claramente $\Theta_{x} M \subseteq 1_{x} M$. Seja $m \in 1_{x} M$, então

$$
m=1_{x} m=\sum_{(x)}\left(\omega_{x} \omega_{x^{-1}}\right) m=\sum_{(x)} \omega_{x}\left(\omega_{x^{-1}} m\right) \in \Theta_{x} M .
$$

Analogamente, $M \Theta_{x}=M 1_{x^{-1}}$, para todo $x \in G$. Assim, dado $m \in \Theta_{x} M$, temos

$$
\left(m_{l} \circ m_{l}^{-1}\right)(m)=\sum_{(x)} \omega_{x} \omega_{x^{-1}} m=1_{x} m=m .
$$

Por outro lado, dados $u_{x} \in \Theta_{x}$ e $m \in M$, temos

$$
\begin{aligned}
\left(m_{l}^{-1} \circ m_{l}\right)\left(u_{x} \otimes m\right) & =\sum_{(x)} \omega_{x} \otimes \omega_{x^{-1}} u_{x} m=\sum_{(x)} \omega_{x} \omega_{x^{-1}} u_{x} \otimes m \\
& =1_{x} u_{x} \otimes m=u_{x} \otimes m .
\end{aligned}
$$

Portanto, $m_{l}$ é isomorfismo de $R$-S-bimódulos. Analogamente, temos que a inversa de $m_{r}$ é dada por

$$
\begin{aligned}
m_{r}^{-1}: M \Theta_{x} & \longrightarrow M \otimes_{R} \Theta_{x} \\
m & \longmapsto \sum_{(x)} m \bar{\omega}_{x^{-1}} \otimes \bar{\omega}_{x},
\end{aligned}
$$

onde $1_{x^{-1}}=\sum_{(\bar{x})} \bar{\omega}_{x^{-1}} \bar{\omega}_{x}$

(iii) Observe que $\Theta_{x} N \subseteq M$ é um $R$-subbimódulo de $M$, tal que:

- $1_{x} n=n$, para todo $n \in \Theta_{x} N$.

- $u_{x^{-1}} n \in N$, para todo $n \in \Theta_{x} N$ e $u_{x^{-1}} \in \Theta_{x^{-1}}$. De fato, se $n \in \Theta_{x} N$, então $n=\sum_{i=1}^{n} u_{x}^{i} n_{i}$, com $u_{x}^{i} \in \Theta_{x}$ e $n_{i} \in N$. Como $N \subseteq M$ é $R$-subbimódulo, temos

$$
u_{x^{-1}} n=\sum_{i=1}^{n} u_{x^{-1}}\left(u_{x}^{i} n_{i}\right)=\sum_{i=1}^{n}(\underbrace{u_{x^{-1}} u_{x}^{i}}_{\in R 1_{x^{-1}}}) n_{i} \in N .
$$


Analogamente, $N \Theta_{x}$ é um $R$-subbimódulo de $M$ que satisfaz:

- $n^{\prime} 1_{x^{-1}}=n$, para todo $n^{\prime} \in N \Theta_{x}$.

- $n^{\prime} u_{x^{-1}} \in N$, para todo $n^{\prime} \in N \Theta_{x}$ e $u_{x^{-1}} \in \Theta_{x^{-1}}$.

Logo, o isomorfismo segue como no item $(i i)$.

Corolário 3.3.12. Seja $N \in \mathcal{S}_{R}(S)$, então $\Theta_{x} \otimes_{R} N \simeq \Theta_{x} N$, para todo $x \in G$. Em particular, $\Theta_{x} \otimes_{R}$ $\Theta_{y} \simeq \Theta_{x} \Theta_{y}$, para todo $x, y \in G$.

Observação 3.3.13. Seja

$$
\begin{aligned}
\Theta: G & \longrightarrow \mathcal{S}_{R}(S) \\
x & \longmapsto \Theta_{x}
\end{aligned}
$$

uma representação unital com $\Theta_{x} \Theta_{x^{-1}}=R 1_{x}$. Pela Proposição 3.3.11 e pelo Corolário 3.3.12 temos que

$$
\begin{aligned}
\underline{\Theta}: G & \longrightarrow \operatorname{PicS}(R) \\
x & \longmapsto\left[\Theta_{x}\right]
\end{aligned}
$$

é uma representação parcial unital com $\Theta_{x} \otimes \Theta_{x^{-1}} \simeq R 1_{x}$, para todo $x \in G$. Seja $f^{\Theta}$ a família de isomorfismos de R-bimódulos onde

$$
\begin{aligned}
f_{x, y}^{\Theta}: \Theta_{x} \otimes_{R} \Theta_{y} & \longrightarrow \Theta_{x} \Theta_{y}=1_{x} \Theta_{x y} \\
u_{x} \otimes u_{y} & \longmapsto u_{x} u_{y}
\end{aligned}
$$

é induzido pela multiplicação em $S$. Então, $f^{\Theta}$ é um conjunto de fatores para $\underline{\Theta}$. Logo, temos um produto cruzado generalizado parcial $\Delta(\Theta)$, onde cada $\Theta_{x} \subseteq S$ é um $R$-subbimodulo.

Reciprocamente, se temos uma representação parcial unital $\Theta: G \longrightarrow \mathbf{P i c S}(R)$, com um conjunto de fatores $f^{\Theta}$, o produto cruzado generalizado parcial $\Delta(\Theta)$ é uma extensão do anel $R$ com mesma unidade. Neste caso, cada $\Theta_{x} \subseteq \Delta(\Theta)$ é um R-subbimódulo. Pelo Corolário 3.3.12 temos que $\underline{\Theta}: G \longrightarrow \mathcal{S}_{R}(\Delta(\Theta))$ com $\underline{\Theta}(x)=\Theta_{x}$, é uma representação parcial unital.

Exemplo 3.3.14. (Produto cruzado parcial) Seja $\alpha=\left(\left\{D_{x}\right\}_{x \in G},\left\{\alpha_{x}\right\}_{x \in G},\left\{\omega_{x, y}\right\}_{x, y \in G}\right)$ uma ação parcial unitária torcida (ver [27, Definição 2.1]) de $G$ sobre $R$ com $D_{x}=R 1_{x}$, para todo $x \in G$. Considere o produto cruzado parcial $R \rtimes_{\alpha, \omega} G=\bigoplus_{x \in G} D_{x} \delta_{x}$ onde o produto é definido por

$$
\left(u_{x} \delta_{x}\right)\left(u_{y} \delta_{y}\right)=u_{x} \alpha_{x}\left(u_{y} 1_{x^{-1}}\right) \omega_{x, y} \delta_{x y} .
$$

Por [27, Teorema 2.4], $R \rtimes_{\alpha, \omega} G$ é associativo. Considere

$$
\begin{aligned}
\Theta: G & \longrightarrow \mathcal{S}_{R}\left(R \rtimes_{\alpha, \omega} G\right) \\
x & \longmapsto D_{x} \delta_{x}
\end{aligned}
$$

É fácil ver que $\left(D_{x} \delta_{x}\right)\left(D_{x^{-1}} \delta_{x^{-1}}\right)\left(D_{x} \delta_{x}\right) \subseteq D_{x} \delta_{x}$, para todo $x \in G$. Por outro lado, seja $u_{x} \delta_{x} \in D_{x} \delta_{x}$, então

$$
\left(u_{x} \delta_{x}\right)\left(1_{x^{-1}} \delta_{x^{-1}}\right)\left(\omega_{x, x^{-1}}^{-1} \delta_{x}\right)=\left(u_{x} \omega_{x, x^{-1}} \delta_{1}\right)\left(\omega_{x, x^{-1}}^{-1} \delta_{x}\right)=u_{x} \omega_{x, x^{-1}} \omega_{x, x^{-1}}^{-1} \delta_{x}=u_{x} 1_{x} \delta_{x}=u_{x} \delta_{x} .
$$

Logo, temos a igualdade $\left(D_{x} \delta_{x}\right)\left(D_{x^{-1}} \delta_{x^{-1}}\right)\left(D_{x} \delta_{x}\right)=D_{x} \delta_{x}$, para todo $x \in G$. Por [27, Lema 5.3], temos que

$$
\left(D_{x} \delta_{x}\right)\left(D_{y} \delta_{y}\right)\left(D_{y^{-1}} \delta_{y^{-1}}\right)=\left(D_{x y} \delta_{x y}\right)\left(D_{y^{-1}} \delta_{y^{-1}}\right) \quad e\left(D_{x}^{-1} \delta_{x}\right)\left(D_{x} \delta_{x}\right)\left(D_{y} \delta_{y}\right)=\left(D_{x^{-1}} \delta_{x^{-1}}\right)\left(D_{x y} \delta_{x y}\right),
$$


para todos $x, y \in G$. Logo, $\Theta$ é representação parcial. Observe agora que $\left(D_{x} \delta_{x}\right)\left(D_{x^{-1}} \delta_{x^{-1}}\right)=D_{x}$, para todo $x \in G$. De fato, a inclusão $\left(D_{x} \delta_{x}\right)\left(D_{x^{-1}} \delta_{x^{-1}}\right) \subseteq D_{x}$ é imediata. Dado $u_{x} \in D_{x}$, temos $\left(u_{x} \omega_{x, x^{-1}}^{-1} \delta_{x}\right)\left(1_{x^{-1}} \delta_{x}\right)=u_{x} \omega_{x, x^{-1}}^{-1} \omega_{x, x^{-1}} \delta_{1}=u_{x} 1_{x} \delta_{1}=u_{x} \delta_{1} \in D_{x}$. Portanto, $\Theta$ é representação parcial unital. Pela Observação 3.3.13, temos que

$$
\begin{aligned}
f_{x, y}^{\Theta}: \quad\left(D_{x} \delta_{x}\right) \otimes\left(D_{y} \delta_{y}\right) & \longrightarrow D_{x} D_{x y} \delta_{x y} \\
u_{x} \delta_{x} \otimes u_{y} \delta_{y} & \longmapsto u_{x} \alpha_{x}\left(u_{y} 1_{x^{-1}}\right) \omega_{x, y} \delta_{x y}
\end{aligned},
$$

é um conjunto de fatores para $\Theta$. Portanto, $\Delta(\Theta)=\bigoplus_{x \in G} D_{x} \delta_{x}=R \rtimes_{\alpha, \omega} G$ é um produto cruzado generalizado parcial.

\subsection{O grupo $\mathcal{C}(\Theta / R)$}

Vamos fixar

$$
\begin{aligned}
& \Theta: \quad G \quad \longrightarrow \quad \operatorname{PicS}(R) \\
& x \longmapsto\left[\Theta_{x}\right]
\end{aligned}
$$

uma representação parcial unital com $\varepsilon_{x}=\Theta_{x} \otimes_{R} \Theta_{x^{-1}} \simeq R 1_{x}$, para todo $x \in G$, tal que exista um conjunto de fatores $f^{\Theta}=\left\{f_{x, y}^{\Theta}: \Theta_{x} \otimes_{R} \Theta_{y} \longrightarrow 1_{x} \Theta_{x y}, x, y \in G\right\}$ para $\Theta$, e seja $\Delta(\Theta)$ o produto cruzado generalizado parcial.

Definimos

$$
\mathcal{C}(\Theta / R)=\left\{[\Delta(\Gamma)] ; \Gamma_{x} \mid \Theta_{x} \text { e } \Gamma_{x} \otimes_{R} \Gamma_{x^{-1}} \simeq R 1_{x}, \text { para todo } x \in G\right\}
$$

Proposição 3.4.1. $\mathcal{C}(\Theta / R)$ é um grupo abeliano com operação definida por

$$
[\Delta(\Omega)][\Delta(\Gamma)]=\left[\bigoplus_{x \in G} \Omega_{x} \otimes_{R} \Theta_{x^{-1}} \otimes_{R} \Gamma_{x}\right] .
$$

com conjunto de fatores dados por:

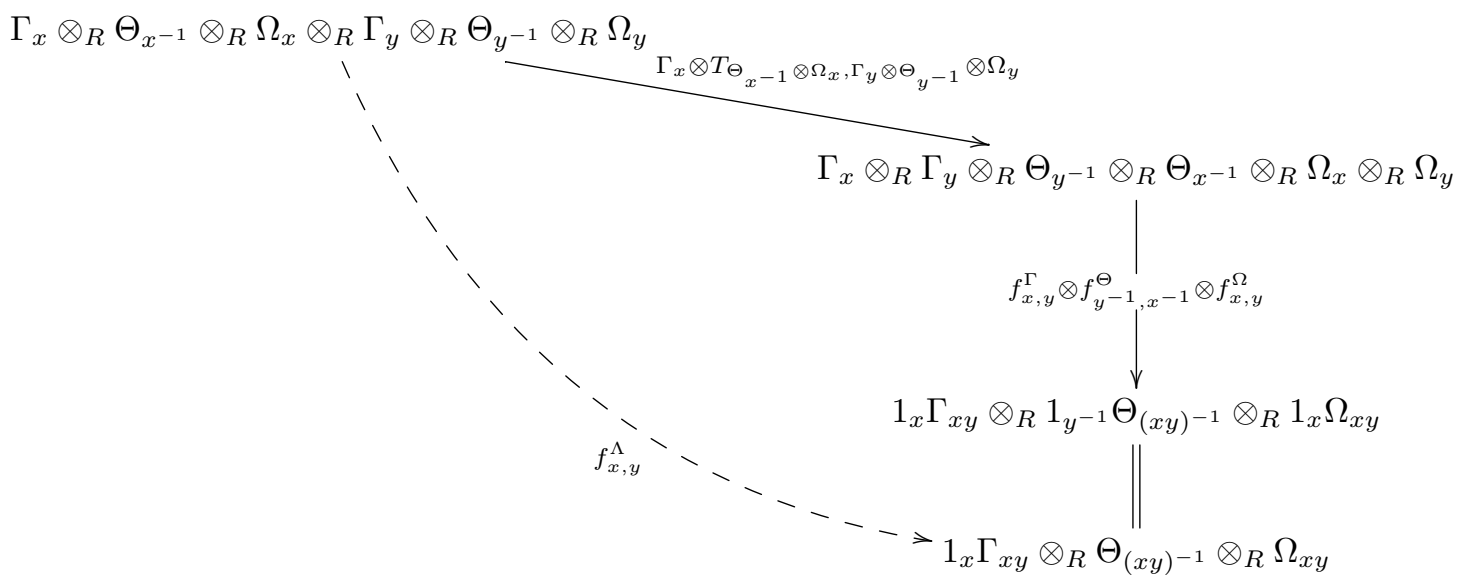

onde $T_{-,-}$é o isomorfismo da Proposição 2.3.5.

Demonstração. Por simplicidade de notação, nessa demonstração $\otimes$ denotará $\otimes_{R}$. Pelo Lema 3.3.8, temos que

$$
[\Delta(\Omega)][\Delta(\Gamma)]=\left[\bigoplus_{x \in G} \Omega_{x} \otimes_{R} \Theta_{x^{-1}} \otimes_{R} \Gamma_{x}\right] \in \mathcal{C}(\Theta / R)
$$


Vamos verificar que essa operação está bem definida, ou seja, não depende da escolha do representante da classe de isomorfismo. Sejam $[\Delta(\Gamma)]=[\Delta(\Sigma)]$ e $[\Delta(\Omega)]=[\Delta(\Lambda)]$ em $\mathcal{C}(\Theta / R)$, então existem isomorfismos de $R$-bimodulos $a_{x}: \Gamma_{x} \longrightarrow \Sigma_{x}$ e $b_{x}: \Omega_{x} \longrightarrow \Lambda_{x}$, tais que os diagramas
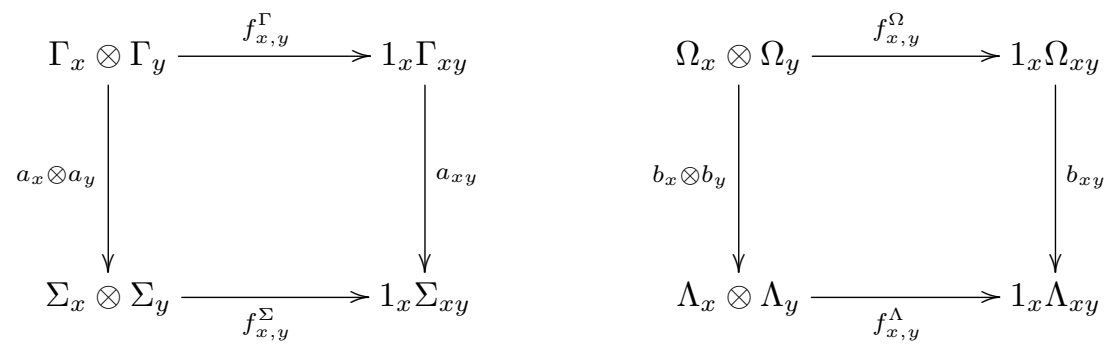

comutam, ou seja,

$$
a_{x y}\left(v_{x} \stackrel{\Gamma}{\circ} v_{y}\right)=\left(a_{x}\left(v_{x}\right) \stackrel{\Sigma}{\circ} a_{y}\left(v_{y}\right)\right) \text { e } b_{x} y\left(\omega_{x} \stackrel{\Omega}{\circ} \omega_{y}\right)=\left(b_{x}\left(\omega_{x}\right) \stackrel{\Lambda}{\circ} b_{y}\left(\omega_{y}\right)\right),
$$

para todo $v_{x} \in \Gamma_{x}, v_{y} \in \Gamma_{y}, \omega_{x} \in \Omega_{x}$ e $\omega_{y} \in \Omega_{y}$. Por definição temos

$$
[\Delta(\Gamma)][\Delta(\Omega)]=\left[\bigoplus_{x \in G} \Gamma_{x} \otimes \Theta_{x^{-1}} \otimes \Omega_{x}\right] \text { e }[\Delta(\Sigma)][\Delta(\Lambda)]=\left[\bigoplus_{x \in G} \Sigma_{x} \otimes \Theta_{x^{-1}} \otimes \Lambda_{x}\right]
$$

Defina o isomorfismo

$$
\begin{aligned}
d_{x y}: \quad \Gamma_{x} \otimes \Theta_{x^{-1}} \otimes \Omega_{x} & \longrightarrow \Sigma_{x} \otimes \Theta_{x^{-1}} \otimes \Lambda_{x} \\
v_{x} \otimes u_{x^{-1}} \otimes \omega_{x} & \longmapsto a_{x}\left(v_{x}\right) \otimes u_{x^{-1}} \otimes b_{x}\left(\omega_{x}\right)
\end{aligned}
$$

Vejamos que o diagrama abaixo é comutativo

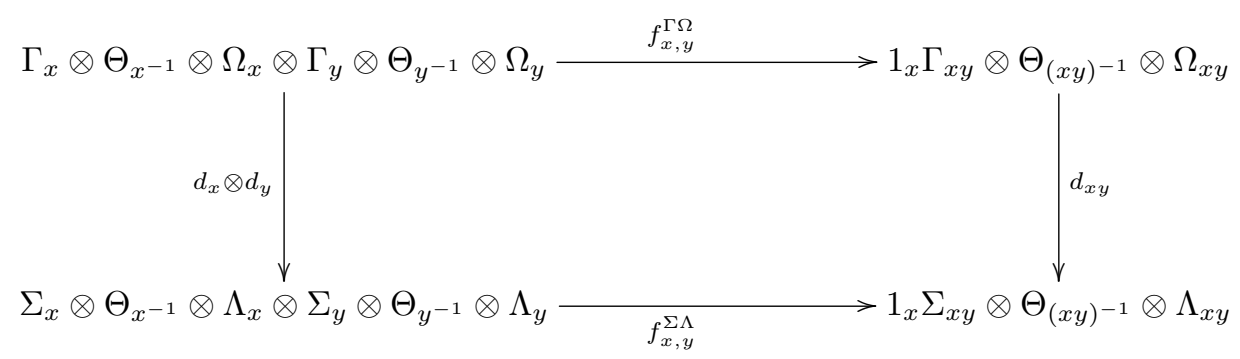

Por um lado, temos que

$$
d_{x y} \circ f_{x, y}^{\Gamma \Omega}=d_{x y} \circ\left(f_{x, y}^{\Gamma} \otimes f_{y^{-1}, x^{-1}}^{\Theta} \otimes f_{x, y}^{\Omega}\right) \circ\left(\Gamma_{x} \otimes T_{1} \otimes \Omega_{y}\right),
$$

onde $T_{1}: \Theta_{x^{-1}} \otimes \Omega_{x} \otimes \Gamma_{y} \otimes \Theta_{y^{-1}} \longrightarrow \Gamma_{y} \otimes \Theta_{y^{-1}} \otimes \Theta_{x^{-1}} \otimes \Omega_{x}$. Por outro lado,

$$
f_{x, y}^{\Sigma \Lambda} \circ\left(d_{x} \otimes d_{y}\right)=\left(f_{x, y}^{\Sigma} \otimes f_{y^{-1}, x^{-1}}^{\Theta} \otimes f_{x, y}^{\Lambda}\right) \circ\left(\Sigma_{x} \otimes T_{2} \otimes \Lambda_{y}\right) \circ\left(d_{x} \otimes d_{y}\right),
$$

onde $T_{2}: \Theta_{x^{-1}} \otimes \Lambda_{x} \otimes \Sigma_{y} \otimes \Theta_{y^{-1}} \longrightarrow \Sigma_{y} \otimes \Theta_{y^{-1}} \otimes \Theta_{x^{-1}} \otimes \Lambda_{x}$. Vamos construir os isomorfismos $T_{1} \mathrm{e}$ $T_{2}$ usando a Proposição 2.3.5. Para isso, considere as aplicações $R$-bilineares $f_{i}: \Theta_{x^{-1}} \otimes \Omega_{x} \longrightarrow R$ e $g_{i}: R \longrightarrow \Theta_{x^{-1}} \otimes \Omega_{x}$, com $i=1,2, \ldots, n$, tais que $\sum_{i=1}^{n} g_{i} \circ f_{i}=I d_{\Theta_{x}-1} \otimes \Omega_{x}$. Defina

$$
\bar{f}_{i}: \Theta_{x^{-1}} \otimes \Lambda_{x} \stackrel{\Theta_{x^{-1}} \otimes b_{x}^{-1}}{\longrightarrow} \Theta_{x^{-1}} \otimes \Omega_{x} \stackrel{f_{i}}{\longrightarrow} R
$$




$$
\bar{g}_{i}: R \stackrel{g_{i}}{\longrightarrow} \Theta_{x^{-1}} \otimes \Omega_{x} \stackrel{\Theta_{x^{-1}} \otimes b_{x}}{\longrightarrow} \Theta_{x^{-1}} \otimes \Lambda_{x}
$$

para $i=1,2, \ldots, n$. Então, $\bar{f}_{i}$ e $\bar{g}_{i}$ são $R$-bilineares e temos $\sum_{i=1}^{n} \bar{g}_{i} \bar{f}_{i}=I d_{\Theta_{x-1} \otimes \Lambda}$. Denotando, $g_{i}(1)=$ $\sum_{l} u_{x^{-1}}^{i, l} \otimes \omega_{x}^{i, l}$, então $\bar{g}_{i}(1)=\sum_{l} u_{x^{-1}}^{i, l} \otimes b_{x}\left(\omega_{x}^{i, l}\right)$. Assim,

$$
\begin{aligned}
T_{1}: \quad \Theta_{x^{-1}} \otimes \Omega_{x} \otimes \Gamma_{y} \otimes \Theta_{y^{-1}} & \longrightarrow \Gamma_{y} \otimes \Theta_{y^{-1}} \otimes \Theta_{x^{-1}} \otimes \Omega_{x} \\
u_{x^{-1}} \otimes \omega_{x} \otimes v_{y} \otimes u_{y^{-1}} & \longmapsto \sum_{i, l} f_{i}\left(u_{x^{-1}} \otimes \omega_{x}\right) v_{y} \otimes u_{y^{-1}} \otimes u_{x^{-1}}^{i, l} \otimes \omega_{x}^{i, l}
\end{aligned}
$$

$T_{2}: \quad \Theta_{x^{-1}} \otimes \Lambda_{x} \otimes \Sigma_{y} \otimes \Theta_{y^{-1}} \longrightarrow \Sigma_{y} \otimes \Theta_{y^{-1}} \otimes \Theta_{x^{-1}} \otimes \Lambda_{x}$

$$
u_{x^{-1}} \otimes l_{x} \otimes t_{y} \otimes u_{y^{-1}} \longmapsto \sum_{i, l} f_{i}\left(u_{x^{-1}} \otimes b_{x}^{-1}\left(l_{x}\right)\right) t_{y} \otimes u_{y^{-1}} \otimes u_{x^{-1}}^{i, l} \otimes b_{x}\left(\omega_{x}^{i, l}\right) .
$$

Logo, $f_{x, y}^{\Sigma \Lambda} \circ\left(d_{x} \otimes d_{y}\right)$ é dado por:

$$
\begin{array}{ll} 
& v_{x} \otimes u_{x^{-1}} \otimes \omega_{x} \otimes v_{y} \otimes u_{y^{-1}} \otimes \omega_{y} \\
\stackrel{d_{x} \otimes d_{y}}{\mapsto} & a_{x}\left(v_{x}\right) \otimes u_{x^{-1}} \otimes b_{x}\left(\omega_{x}\right) \otimes a_{y}\left(v_{y}\right) \otimes u_{y^{-1}} \otimes b_{y}\left(\omega_{y}\right) \\
\stackrel{T_{2}}{\mapsto} & \sum_{i, l} a_{x}\left(v_{x}\right) \otimes f_{i}\left(u_{x^{-1}} \otimes \omega_{x}\right) a_{y}\left(v_{y}\right) \otimes u_{y^{-1}} \otimes u_{x^{-1}}^{i, l} \otimes b_{x}\left(\omega_{x}^{i, l}\right) \otimes b_{y}\left(\omega_{y}\right) \\
\mapsto & \sum_{i, l}\left(a_{x}\left(v_{x} \stackrel{\Sigma}{\circ} f_{i}\left(u_{x^{-1}} \otimes \omega_{x}\right) a_{y}\left(v_{y}\right)\right) \otimes\left(u_{y^{-1}} \stackrel{\ominus}{\circ} u_{x^{-1}}^{i, l}\right) \otimes\left(b_{x}\left(\omega_{x}^{i, l}\right) \stackrel{\Lambda}{\circ} b_{y}\left(\omega_{y}\right)\right) .\right.
\end{array}
$$

Por outro lado, $d_{x y} \circ f_{x, y}^{\Gamma \Omega}$ :

$$
\begin{aligned}
& v_{x} \otimes u_{x^{-1}} \otimes \omega_{x} \otimes v_{y} \otimes u_{y^{-1}} \otimes \omega_{y} \\
& \stackrel{T_{7}}{\mapsto} \quad \sum_{i, l} v_{x} \otimes f_{i}\left(u_{x^{-1}} \otimes \omega_{x}\right) v_{y} \otimes u_{y^{-1}} \otimes u_{x^{-1}}^{i, l} \otimes \omega_{x}^{i, l} \otimes \omega_{y}
\end{aligned}
$$

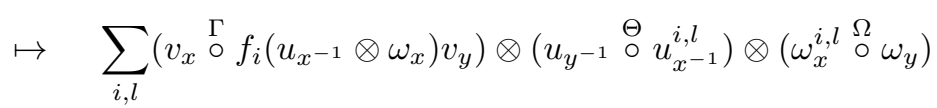

$$
\begin{aligned}
& \stackrel{d_{x y}}{\mapsto} \sum_{i, l} a_{x y}\left(v_{x} \stackrel{\Gamma}{\circ} f_{i}\left(u_{x^{-1}} \otimes \omega_{x}\right) v_{y}\right) \otimes\left(u_{y^{-1}} \stackrel{\ominus}{\circ} u_{x^{-1}}^{i, l}\right) \otimes b_{x y}\left(\omega_{x}^{i, l} \stackrel{\Omega}{\circ} \omega_{y}\right) \\
& \stackrel{(3.33)}{=} \sum_{i, l}\left(a_{x}\left(v_{x}\right) \stackrel{\Sigma}{\circ} f_{i}\left(u_{x^{-1}} \otimes \omega_{x}\right) a_{y}\left(v_{y}\right)\right) \otimes\left(u_{y^{-1}} \stackrel{\ominus}{\circ} u_{x^{-1}}^{i, l}\right) \otimes\left(b_{x}\left(\omega_{x}^{i, l}\right) \stackrel{\Lambda}{\circ} b_{y}\left(\omega_{y}\right)\right) \text {. }
\end{aligned}
$$

Segue então que $d_{x y} \circ f_{x, y}^{\Gamma \Omega}=f_{x, y}^{\Sigma \Lambda} \circ\left(d_{x} \otimes d_{y}\right)$, para todo $x, y \in G$. Isso implica que

$$
[\Delta(\Gamma)][\Delta(\Omega)]=[\Delta(\Sigma)][\Delta(\Lambda)] \text { em } \mathcal{C}(\Theta / R) .
$$

Portanto, a operação está bem definida.

Vejamos que, com essa operação, $\mathcal{C}(\Theta / R)$ é de fato um grupo.

Associatividade: Considere $[\Delta(\Gamma)],[\Delta(\Omega)],[\Delta(\Sigma)] \in \mathcal{C}(\Theta / R)$. Então,

e

$$
([\Delta(\Gamma)][\Delta(\Omega)])[\Delta(\Sigma)]=\left[\bigoplus_{x \in G} \Gamma_{x} \otimes \Theta_{x^{-1}} \otimes \Omega_{x} \otimes \Theta_{x^{-1}} \otimes \Sigma_{x}\right]
$$

$$
[\Delta(\Gamma)]([\Delta(\Omega)][\Delta(\Sigma)])=\left[\bigoplus_{x \in G} \Gamma_{x} \otimes \Theta_{x^{-1}} \otimes \Omega_{x} \otimes \Theta_{x^{-1}} \otimes \Sigma_{x}\right]
$$


Vamos mostrar que $f_{x, y}^{(\Gamma \Omega) \Sigma}=f_{x, y}^{\Gamma(\Omega \Sigma)}$, para todo $x, y \in G$. Por um lado temos que $f_{x, y}^{(\Gamma \Omega) \Sigma}$ é dada pela $\Gamma_{x} \otimes \Theta_{x^{-1}} \otimes \Omega_{x} \otimes \Theta_{x^{-1}} \otimes \Sigma_{x} \otimes \Gamma_{y} \otimes \Theta_{y^{-1}} \otimes \Omega_{y} \otimes \Theta_{y^{-1}} \otimes \Sigma_{y}$

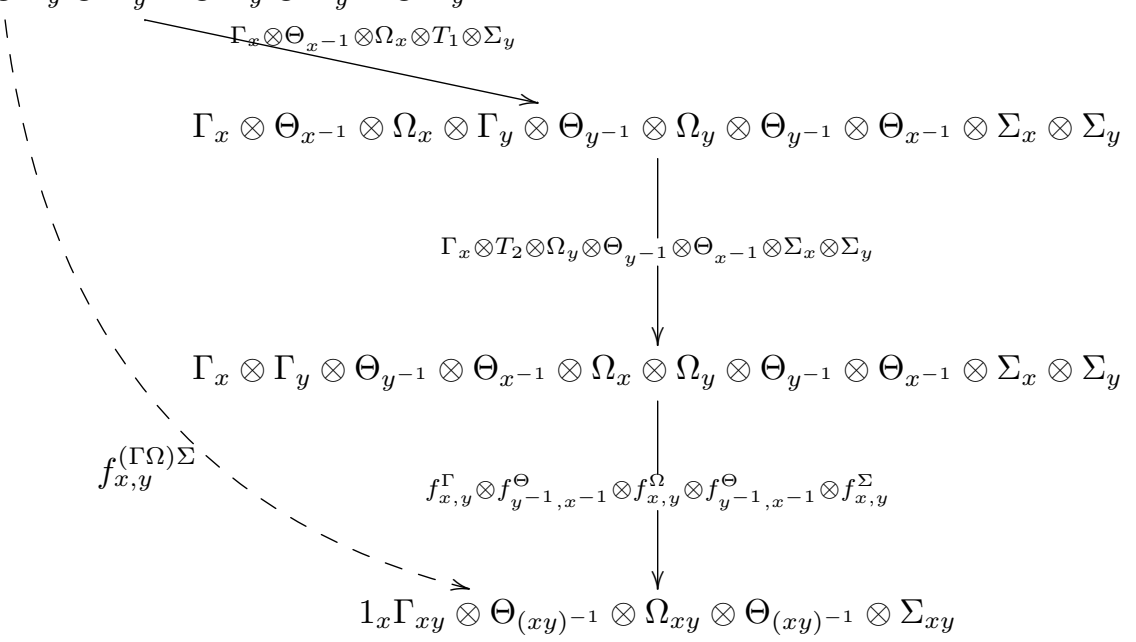

onde $T_{1}$ e $T_{2}$ são os isomorfismos

$$
\begin{gathered}
T_{1}: \Theta_{x^{-1}} \otimes \Sigma_{x} \otimes \Gamma_{y} \otimes \Theta_{y^{-1}} \otimes \Omega_{y} \otimes \Theta_{y^{-1}} \longrightarrow \Gamma_{y} \otimes \Theta_{y^{-1}} \otimes \Omega_{y} \otimes \Theta_{y^{-1}} \otimes \Theta_{x^{-1}} \otimes \Sigma_{x} \\
T_{2}: \Theta_{x^{-1}} \otimes \Omega_{x} \otimes \Gamma_{y} \otimes \Theta_{y^{-1}} \longrightarrow \Gamma_{y} \otimes \Theta_{y^{-1}} \otimes \Theta_{x^{-1}} \otimes \Omega_{x}
\end{gathered}
$$

Por outro lado, $f_{x, y}^{\Gamma(\Omega \Sigma)}$ é dado por

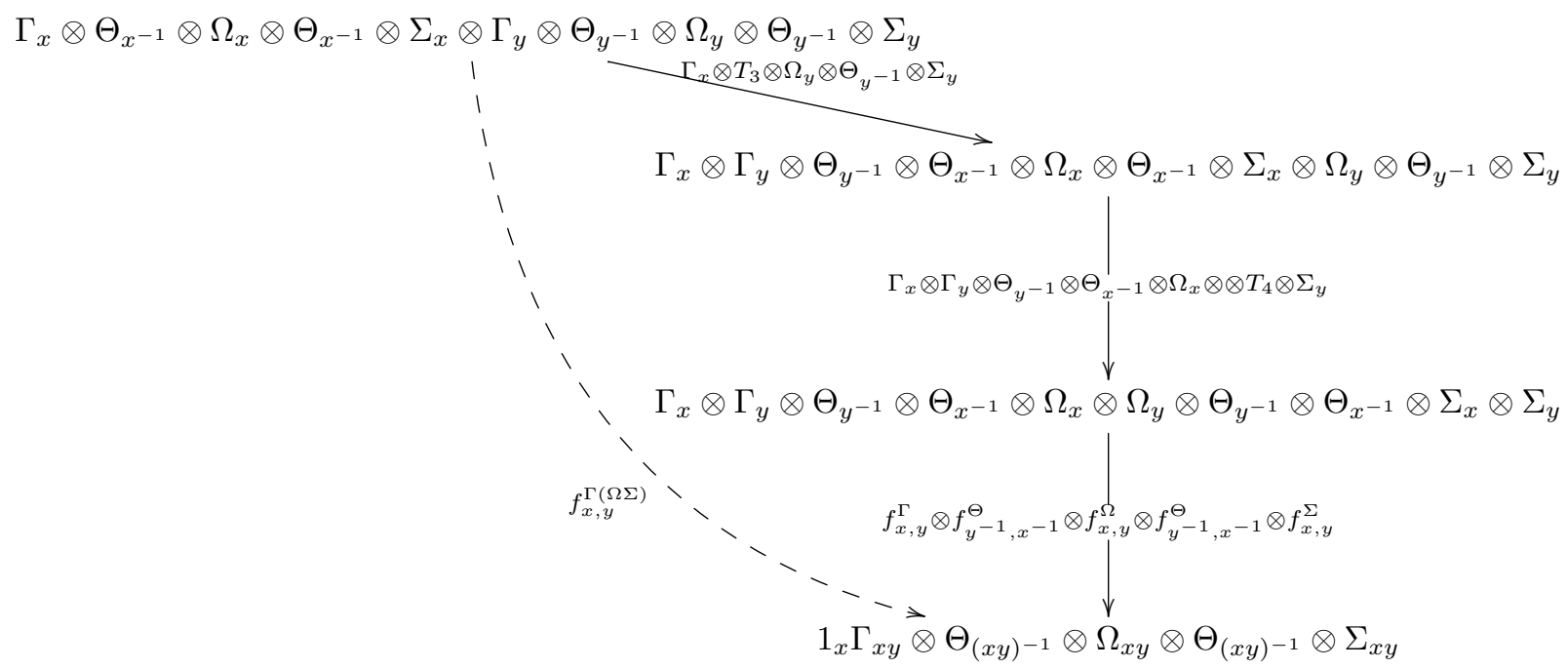

onde

$$
\begin{gathered}
T_{3}: \Theta_{x^{-1}} \otimes \Omega_{x} \otimes \Theta_{x^{-1}} \otimes \Sigma_{x} \otimes \Gamma_{y} \otimes \Theta_{y^{-1}} \longrightarrow \Gamma_{y} \otimes \Theta_{y^{-1}} \otimes \Theta_{x^{-1}} \otimes \Omega_{x} \otimes \Theta_{x^{-1}} \otimes \Sigma_{x} \\
T_{4}: \Theta_{x^{-1}} \otimes \Sigma_{x} \otimes \Omega_{y} \otimes \Theta_{y^{-1}} \longrightarrow \Omega_{y} \otimes \Theta_{y^{-1}} \otimes \Theta_{x^{-1}} \otimes \Sigma_{x} .
\end{gathered}
$$

Vamos construir os isomorfismos $T_{1}, T_{2}, T_{3}$ e $T_{4}$ : Sejam $f_{i}: \Theta_{x^{-1}} \otimes \Sigma_{x} \longrightarrow R$ e $g_{i}: R \longrightarrow \Theta_{x^{-1}} \otimes \Sigma_{x}$, com $i=1,2, \ldots, n$, aplicações $R$-bilineares que satisfazem $\sum_{i=1}^{n} g_{i} f_{i}=I d_{\Theta_{x^{-1}} \otimes \Sigma_{x}}$. Denote $g_{i}(1)=\sum_{l} u_{x^{-1}}^{i, l} \otimes t_{x}^{i, l}$, 
então

$$
\begin{aligned}
T_{1}: \quad \Theta_{x^{-1}} \otimes \Sigma_{x} \otimes \Gamma_{y} \otimes \Theta_{y^{-1}} \otimes \Omega_{y} \otimes \Theta_{y^{-1}} & \longrightarrow \Gamma_{y} \otimes \Theta_{y^{-1}} \otimes \Omega_{y} \otimes \Theta_{y^{-1}} \otimes \Theta_{x^{-1}} \otimes \Sigma_{x} \\
u_{x^{-1}} \otimes t_{x} \otimes v_{y} \otimes u_{y^{-1}} \otimes \omega_{y} \otimes u_{y^{-1}}^{\prime} & \longmapsto \sum_{i, l} f_{i}\left(u_{x^{-1}} \otimes t_{x}\right) v_{y} \otimes u_{y^{-1}} \otimes \omega_{y} \otimes u_{y^{-1}}^{\prime} \otimes u_{x^{-1}}^{i, l} \otimes t_{x}^{i, l},
\end{aligned}
$$

e

$$
\begin{aligned}
T_{4}: \quad \Theta_{x^{-1}} \otimes \Sigma_{x} \otimes \Omega_{y} \otimes \Theta_{y^{-1}} & \longrightarrow \Omega_{y} \otimes \Theta_{y^{-1}} \otimes \Theta_{x^{-1}} \otimes \Sigma_{x} \\
u_{x^{-1}} \otimes t_{x} \otimes \omega_{y} \otimes u_{y^{-1}} & \longmapsto \sum_{i, l} f_{i}\left(u_{x^{-1}} \otimes t_{x}\right) \omega_{y} \otimes u_{y^{-1}} \otimes u_{x^{-1}}^{i, l} \otimes t_{x}^{i, l} .
\end{aligned}
$$

Considere agora $f_{j}^{\prime}: \Gamma_{y} \otimes \Theta_{y^{-1}} \longrightarrow R$ e $g_{j}^{\prime}: R \longrightarrow \Gamma_{y} \otimes \Theta_{y^{-1}}$, com $j=1,2, \ldots, m$, aplicações $R$-bilinear que satisfazem $\sum_{j=1}^{m} g_{j}^{\prime} f_{j}^{\prime}=I d_{\Gamma_{y} \otimes \Theta_{y^{-1}}}$. Denote $g_{j}(1)=\sum_{k} \widetilde{v}_{y}^{j, k} \otimes \widetilde{u}_{y^{-1}}^{j, k}$, então

$$
\begin{aligned}
T_{2}: \quad \Theta_{x^{-1}} \otimes \Omega_{x} \otimes \Gamma_{y} \otimes \Theta_{y^{-1}} & \longrightarrow \Gamma_{y} \otimes \Theta_{y^{-1}} \otimes \Theta_{x^{-1}} \otimes \Omega_{x} \\
u_{x^{-1}} \otimes \omega_{x} \otimes v_{y} \otimes u_{y^{-1}} & \longmapsto \sum_{j, k} \widetilde{v}_{y}^{j, k} \otimes \widetilde{u}_{y^{-1}}^{j, k} \otimes u_{x^{-1}} \otimes \omega_{x} f_{j}^{\prime}\left(v_{y} \otimes u_{y^{-1}}\right)
\end{aligned}
$$

e

$$
\begin{aligned}
T_{3}: \quad \Theta_{x^{-1}} \otimes \Omega_{x} \otimes \Theta_{x^{-1}} \otimes \Sigma_{x} \otimes \Gamma_{y} \otimes \Theta_{y^{-1}} & \longrightarrow \Gamma_{y} \otimes \Theta_{y^{-1}} \otimes \Theta_{x^{-1}} \otimes \Omega_{x} \otimes \Theta_{x^{-1}} \otimes \Sigma_{x} \\
u_{x^{-1}} \otimes \omega_{x} \otimes u_{x^{-1}}^{\prime} \otimes t_{x} \otimes v_{y} \otimes u_{y^{-1}} & \longmapsto \sum_{j, k} \widetilde{v}_{y}^{j, k} \otimes \widetilde{u}_{y^{-1}}^{j, k} \otimes u_{x^{-1}} \otimes \omega_{x} \otimes u_{x^{-1}}^{\prime} \otimes t_{x} f_{j}^{\prime}\left(v_{y} \otimes u_{y^{-1}}\right)
\end{aligned}
$$

Vamos calcular $f_{x, y}^{(\Gamma \Omega) \Sigma}$ :

$$
\begin{aligned}
& v_{x} \otimes u_{x^{-1}} \otimes \omega_{x} \otimes u_{x^{-1}}^{\prime} \otimes t_{x} \otimes v_{y} \otimes u_{y^{-1}} \otimes \omega_{y} \otimes u_{y^{-1}}^{\prime} \otimes t_{y} \\
& \stackrel{T_{1}}{\mapsto} \sum_{i, l} v_{x} \otimes u_{x^{-1}} \otimes \omega_{x} \otimes f_{i}\left(u_{x^{-1}}^{\prime} \otimes t_{x}\right) v_{y} \otimes u_{y^{-1}} \otimes \omega_{y} \otimes u_{y^{-1}}^{\prime} \otimes u_{x^{-1}}^{i, l} \otimes t_{x}^{i, l} \otimes t_{y} \\
& \stackrel{T_{2}}{\mapsto} \sum_{i, j, l, k} v_{x} \otimes \widetilde{v}_{y}^{j, k} \otimes \widetilde{u}_{y^{-1}}^{j, k} \otimes u_{x^{-1}} \otimes \omega_{x} f_{j}^{\prime}\left(f_{i}\left(u_{x^{-1}}^{\prime} \otimes t_{x}\right) v_{y} \otimes u_{y^{-1}}\right) \otimes \omega_{y} \otimes u_{y^{-1}}^{\prime} \otimes u_{x^{-1}}^{i, l} \otimes t_{x}^{i, l} \otimes t_{y} \\
& \mapsto \sum_{i, j, l, k}\left(v_{x} \stackrel{\Gamma}{\circ} \widetilde{v}_{y}^{j, k}\right) \otimes\left(\widetilde{u}_{y^{-1}}^{j, k} \stackrel{\ominus}{\circ} u_{x^{-1}}\right) \otimes\left(\omega_{x} f_{j}^{\prime}\left(f_{i}\left(u_{x^{-1}}^{\prime} \otimes t_{x}\right) v_{y} \otimes u_{y^{-1}}\right) \stackrel{\Omega}{\circ} \omega_{y}\right) \otimes\left(u_{y^{-1}}^{\prime} \stackrel{\ominus}{\circ} u_{x^{-1}}^{i, l}\right) \otimes\left(t_{x}^{i, l} \stackrel{\ominus}{\circ} t_{y}\right) .
\end{aligned}
$$

Por outro lado, para $f_{x, y}^{\Gamma(\Omega \Sigma)}$ temos:

$$
\begin{aligned}
& v_{x} \otimes u_{x^{-1}} \otimes \omega_{x} \otimes u_{x^{-1}}^{\prime} \otimes t_{x} \otimes v_{y} \otimes u_{y^{-1}} \otimes \omega_{y} \otimes u_{y^{-1}}^{\prime} \otimes t_{y} \\
& \stackrel{T_{3}}{\mapsto} \sum_{j, k} v_{x} \otimes \widetilde{v}_{y}^{j, k} \otimes \widetilde{u}_{y^{-1}}^{j, k} \otimes u_{x^{-1}} \otimes \omega_{x} \otimes u_{x^{-1}}^{\prime} \otimes t_{x} f_{j}^{\prime}\left(v_{y} \otimes u_{y^{-1}}\right) \otimes \omega_{y} \otimes u_{y^{-1}}^{\prime} \otimes t_{y} \\
& \stackrel{T_{4}}{\mapsto} \sum_{i, j, k, l} v_{x} \otimes \widetilde{v}_{y}^{j, k} \otimes \widetilde{u}_{y^{-1}}^{j, k} \otimes u_{x^{-1}} \otimes \omega_{x} \otimes f_{i}\left(u_{x^{-1}}^{\prime} \otimes t_{x} f_{j}^{\prime}\left(v_{y} \otimes u_{y^{-1}}\right)\right) \omega_{y} \otimes u_{y^{-1}}^{\prime} \otimes u_{x^{-1}}^{i, l} \otimes t_{x}^{i, l} \otimes t_{y} \\
& =\sum_{i, j, k, l} v_{x} \otimes \widetilde{v}_{y}^{j, k} \otimes \widetilde{u}_{y^{-1}}^{j, k} \otimes u_{x^{-1}} \otimes \omega_{x} \otimes f_{i}\left(u_{x^{-1}}^{\prime} \otimes t_{x}\right) f_{j}^{\prime}\left(v_{y} \otimes u_{y^{-1}}\right) \omega_{y} \otimes u_{y^{-1}}^{\prime} \otimes u_{x^{-1}}^{i, l} \otimes t_{x}^{i, l} \otimes t_{y} \\
& =\sum_{i, j, k, l} v_{x} \otimes \widetilde{v}_{y}^{j, k} \otimes \widetilde{u}_{y^{-1}}^{j, k} \otimes u_{x^{-1}} \otimes \omega_{x} \otimes f_{j}^{\prime}\left(f_{i}\left(u_{x^{-1}}^{\prime} \otimes t_{x}\right) v_{y} \otimes u_{y^{-1}}\right) \omega_{y} \otimes u_{y^{-1}}^{\prime} \otimes u_{x^{-1}}^{i, l} \otimes t_{x}^{i, l} \otimes t_{y} \\
& \mapsto \sum_{i, j, l, k}\left(v_{x} \stackrel{\circ}{\circ} \widetilde{v}_{y}^{j, k}\right) \otimes\left(\widetilde{u}_{y^{-1}}^{j, k} \stackrel{\ominus}{\circ} u_{x^{-1}}\right) \otimes\left(\omega_{x} f_{j}^{\prime}\left(f_{i}\left(u_{x^{-1}}^{\prime} \otimes t_{x}\right) v_{y} \otimes u_{y^{-1}}\right) \stackrel{\Omega}{\circ} \omega_{y}\right) \otimes\left(u_{y^{-1}}^{\prime} \stackrel{\ominus}{\circ} u_{x^{-1}}^{i, l}\right) \otimes\left(t_{x}^{i, l} \stackrel{\ominus}{\circ} t_{y}\right) .
\end{aligned}
$$

Logo, $f_{x, y}^{(\Gamma \Omega) \Sigma}=f_{x, y}^{\Gamma(\Omega \Sigma)}$. Portanto, temos a associatividade. 
Elemento neutro: Vejamos agora que o elemento neutro é $[\Delta(\Theta)]$. Dado $[\Delta(\Omega)] \in \mathcal{C}(\Theta / R)$, temos

$$
[\Delta(\Omega)][\Delta(\Theta)]=\left[\bigoplus_{x \in G} \Omega_{x} \otimes_{R} \Theta_{x^{-1}} \otimes_{R} \Theta_{x}\right] .
$$

Para cada $x \in G$ considere o isomorfismo de $R$-bimódulos

$$
\begin{aligned}
\varphi_{x}: \Omega_{x} \otimes_{R} \Theta_{x} \otimes_{R} \Theta_{x^{-1}} & \longrightarrow \Omega_{x} \\
\omega_{x} \otimes u_{x} \otimes u_{x^{-1}} & \longmapsto \omega_{x}\left(u_{x} \stackrel{\ominus}{\circ} u_{x^{-1}}\right)
\end{aligned}
$$

Então, o temos o diagrama comutativo

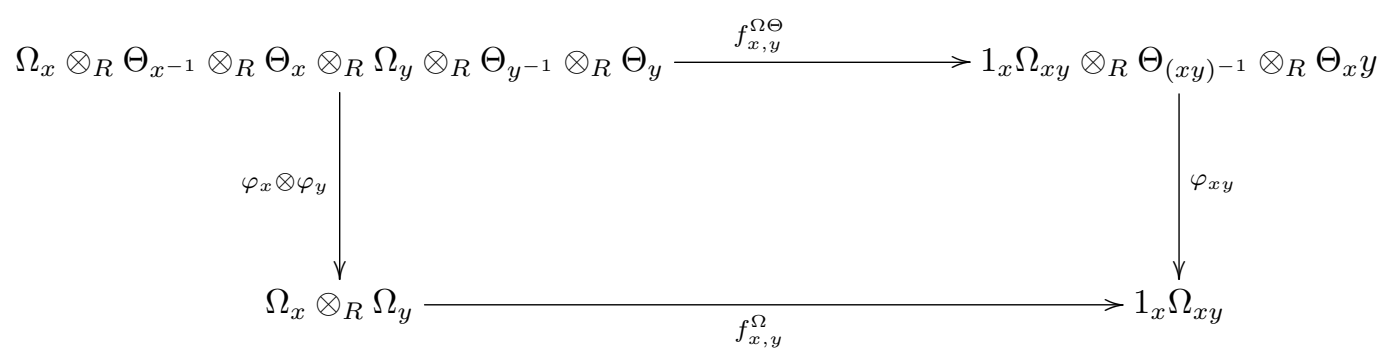

De fato, por um lado, temos:

$$
f_{x, y}^{\Omega} \circ\left(\varphi_{x} \otimes \varphi_{y}\right)\left(\omega_{x} \otimes u_{x^{-1}} \otimes u_{x} \otimes \omega_{y} \otimes u_{y^{-1}} \otimes u_{y}\right)=\left(\omega_{x}\left(u_{x^{-1}} \stackrel{\ominus}{\circ} u_{x}\right) \stackrel{\Omega}{\circ} \omega_{y}\left(u_{y^{-1}} \stackrel{\ominus}{\circ} u_{y}\right)\right) .
$$

Para o outro lado, observe que $\varphi_{x y} \circ f_{x, y}^{\Omega \Theta}$ é dado por

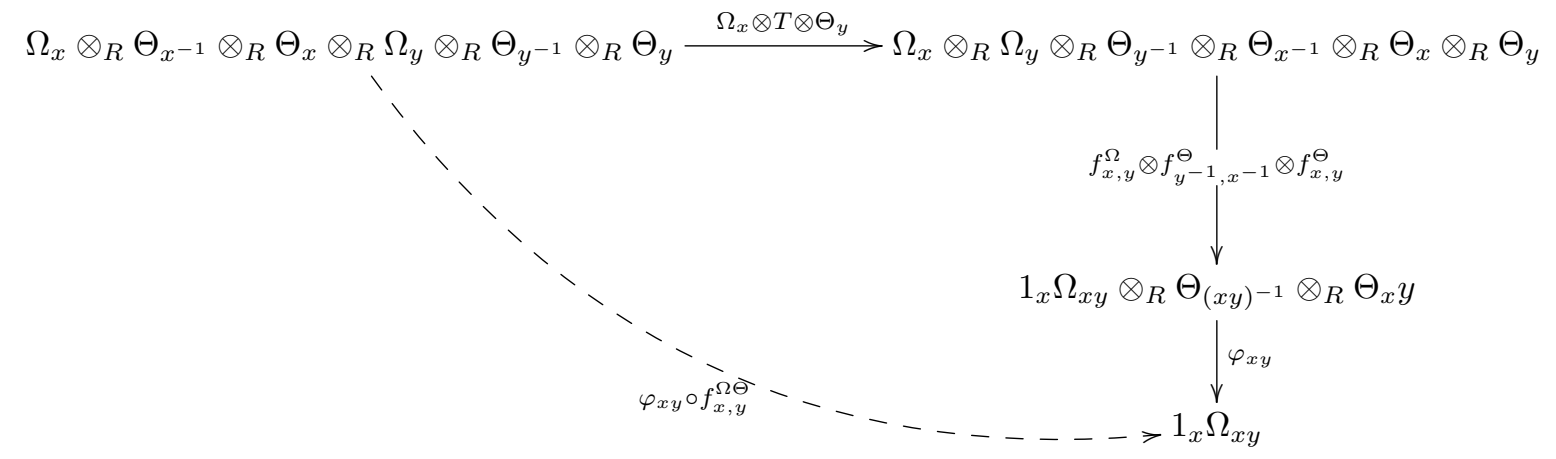

onde $T: \Theta_{x^{-1}} \otimes_{R} \Theta_{x} \otimes \Omega_{y} \otimes_{R} \Theta_{y^{-1}} \longrightarrow \Omega_{y} \otimes_{R} \Theta_{y^{-1}} \otimes \Theta_{x^{-1}} \otimes_{R} \Theta_{x}$ é um isomorfismo de $R$-bimódulos como na Proposição 2.3.5. Considere os isomorfismos de $R$-bimódulos

$$
\begin{gathered}
f: \Theta_{x^{-1}} \otimes \Theta_{x} \stackrel{f_{x^{-1}, x}^{\Theta} \longrightarrow R 1_{x^{-1}} \hookrightarrow}{\longrightarrow} R \\
g: R \longrightarrow R 1_{x^{-1}} \stackrel{\left(f_{x^{-1}, x}^{\Theta}\right)^{-1}}{\longrightarrow} \Theta_{x^{-1}} \otimes \Theta_{x}
\end{gathered}
$$

É fácil ver que $g f=I d_{\Theta_{x-1} \otimes \Theta_{x}}$. Então, pelo Lema 2.3.5, o isomorfismo $T$ é dado por

$$
\begin{aligned}
T: \Theta_{x^{-1}} \otimes \Theta_{x} \otimes \Omega_{y} \otimes \Theta_{y^{-1}} & \longrightarrow \Omega_{y} \otimes \Theta_{y^{-1}} \otimes \Theta_{x^{-1}} \otimes \Theta_{x} \\
u_{x^{-1}} \otimes u_{x} \otimes \omega_{y} \otimes u_{y^{-1}} & \longmapsto \sum_{(x)}\left(u_{x^{-1}} \stackrel{\ominus}{\circ} u_{x}\right) \omega_{y} \otimes u_{y^{-1}} \otimes \widetilde{u}_{x^{-1}} \otimes \widetilde{u}_{x},
\end{aligned}
$$


onde $\sum_{(x)}\left(\widetilde{u}_{x^{-1}} \stackrel{\ominus}{\circ} \widetilde{u}_{x}\right)=1_{x^{-1}}$

Assim, $\varphi_{x y} \circ f_{x, y}^{\Omega \Theta}$ é dado por:

$$
\begin{aligned}
& \omega_{x} \otimes u_{x^{-1}} \otimes u_{x} \otimes \omega_{y} \otimes u_{y^{-1}} \otimes u_{y} \mapsto \sum_{(x)} \omega_{x} \otimes\left(u_{x^{-1}} \stackrel{\ominus}{\circ} u_{x}\right) \omega_{y} \otimes u_{y^{-1}} \otimes \widetilde{u}_{x^{-1}} \otimes \widetilde{u}_{x} \otimes u_{y}
\end{aligned}
$$

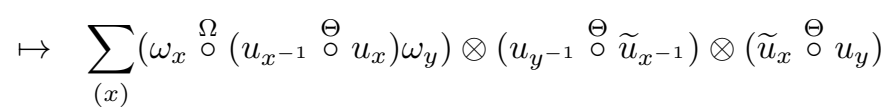

$$
\begin{aligned}
& \mapsto \sum_{(x)}\left(\omega_{x} \stackrel{\Omega}{\circ}\left(u_{x^{-1}} \stackrel{\ominus}{\circ} u_{x}\right) \omega_{y}\right)\left(\left(u_{y^{-1}} \stackrel{\ominus}{\circ} \widetilde{u}_{x^{-1}}\right) \stackrel{\ominus}{\circ}\left(\widetilde{u}_{x} \stackrel{\ominus}{\circ} u_{y}\right)\right) \\
& =\sum_{(x)}\left(\omega_{x} \stackrel{\Omega}{\circ}\left(u_{x^{-1}} \stackrel{\ominus}{\circ} u_{x}\right) \omega_{y}\right)\left(u_{y^{-1}} \stackrel{\ominus}{\circ}\left(\widetilde{u}_{x^{-1}} \stackrel{\ominus}{\circ} \widetilde{u}_{x}\right) \stackrel{\ominus}{\circ} u_{y}\right) \\
& =\left(\omega_{x} \stackrel{\Omega}{\circ}\left(u_{x^{-1}} \stackrel{\ominus}{\circ} u_{x}\right) \omega_{y}\right)\left(u_{y^{-1}} 1_{x^{-1}} \stackrel{\ominus}{\circ} u_{y}\right) \\
& =\left(\omega_{x} \stackrel{\Omega}{\circ}\left(u_{x^{-1}} \stackrel{\ominus}{\circ} u_{x}\right) \omega_{y}\left(1_{(x y)^{-1}} u_{y^{-1}} \stackrel{\ominus}{\circ} u_{y}\right)\right) \\
& =\left(\omega_{x} \stackrel{\Omega}{\circ}\left(u_{x^{-1}} \stackrel{\ominus}{\circ} u_{x} 1_{x^{-1}}\right) \omega_{y}\left(u_{y^{-1}} \stackrel{\ominus}{\circ} u_{y}\right)\right) \\
& =\left(\omega_{x}\left(u_{x^{-1}} \stackrel{\ominus}{\circ} u_{x}\right) \stackrel{\Omega}{\circ} \omega_{y}\left(u_{y^{-1}} \stackrel{\ominus}{\circ} u_{y}\right)\right) \text {. }
\end{aligned}
$$

Logo, $f_{x, y}^{\Omega} \circ\left(\varphi_{x} \otimes \varphi_{y}\right)=\varphi_{x y} \circ f_{x, y}^{\Omega \Theta}$. Portanto, temos a afirmação. A comutatividade do diagrama implica que

$$
[\Delta(\Omega)][\Delta(\Theta)]=[\Delta(\Omega)] \text { em } \mathcal{C}(\Theta / R)
$$

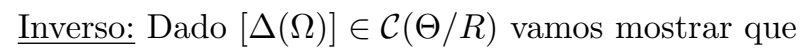

$$
[\Delta(\Omega)]^{-1}=\left[\bigoplus_{x \in G} \Theta_{x} \otimes_{R} \Omega_{x^{-1}} \otimes_{R} \Theta_{x}\right] .
$$

Pelo Lema 3.3.8 temos que $[\Delta(\Omega)]^{-1} \in \mathcal{C}(\Theta / R)$. Vamos verificar que

$$
[\Delta(\Omega)][\Delta(\Omega)]^{-1}=\left[\bigoplus_{x \in G} \Omega_{x} \otimes_{R} \Theta_{x^{-1}} \otimes_{R} \Theta_{x} \otimes_{R} \Omega_{x^{-1}} \otimes_{R} \Theta_{x}\right]=[\Delta(\Theta)]
$$

Considere $\psi_{x}$ o isomorfismo de $R$-bimódulos dado por

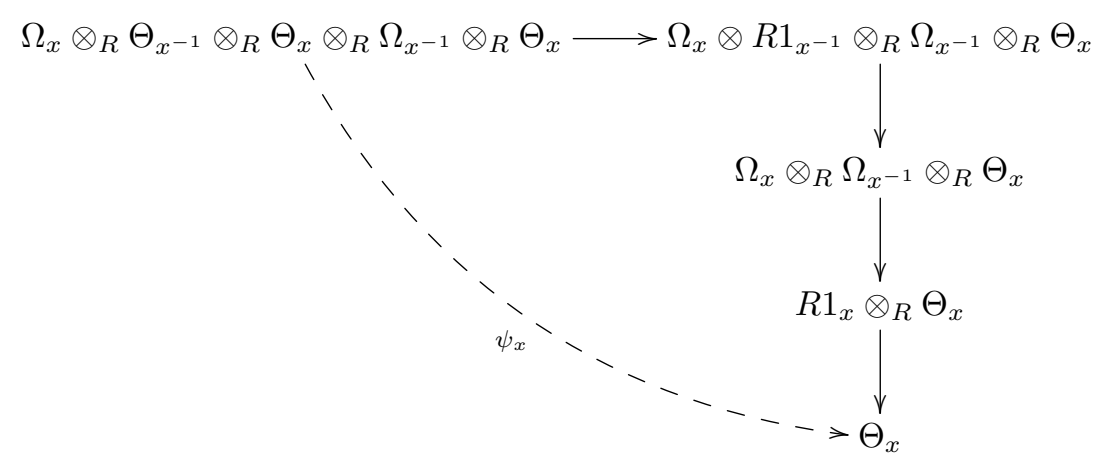

ou seja,

$$
\psi_{x}\left(\omega_{x} \otimes u_{x^{-1}} \otimes u_{x} \otimes \omega_{x^{-1}} \otimes u_{x}^{\prime}\right)=\left(\omega_{x}\left(u_{x^{-1}} \stackrel{\ominus}{\circ} u_{x}\right) \stackrel{\Omega}{\circ} \omega_{x^{-1}}\right) u_{x}^{\prime} .
$$


Denotando $\Pi_{x}=\Omega_{x} \otimes_{R} \Theta_{x^{-1}} \otimes_{R} \Theta_{x} \otimes_{R} \Omega_{x^{-1}} \otimes_{R} \Theta_{x}$, para todo $x \in G$, vamos verificar que o diagrama

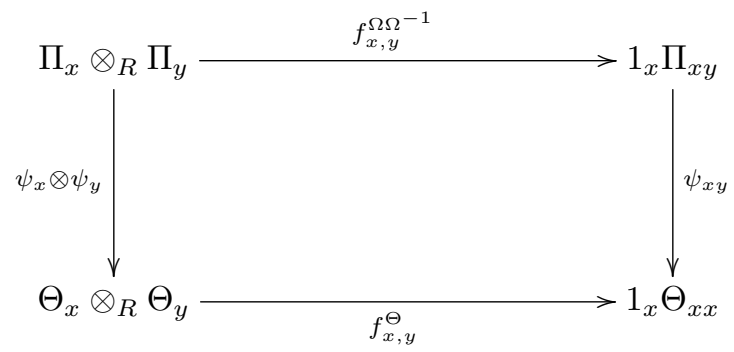

é comutativo. Por um lado, temos

$$
\begin{aligned}
& \left(f_{x, y}^{\Theta} \circ\left(\psi_{x} \otimes \psi_{y}\right)\right)\left(\omega_{x} \otimes u_{x^{-1}} \otimes u_{x} \otimes \omega_{x^{-1}} \otimes u_{x}^{\prime} \otimes \omega_{y} \otimes u_{y^{-1}} \otimes u_{y} \otimes \omega_{y^{-1}} \otimes u_{y}^{\prime}\right) \\
= & \left(\left(\omega_{x}\left(u_{x^{-1}} \stackrel{\ominus}{\circ} u_{x}\right) \stackrel{\Omega}{\circ} \omega_{x^{-1}}\right) u_{x}^{\prime} \stackrel{\ominus}{\circ}\left(\omega_{y}\left(u_{y^{-1}} \stackrel{\ominus}{\circ} u_{y}\right) \stackrel{\Omega}{\circ} \omega_{y^{-1}}\right) u_{y}^{\prime}\right) .
\end{aligned}
$$

O outro lado do diagrama é dado pelas aplicações:

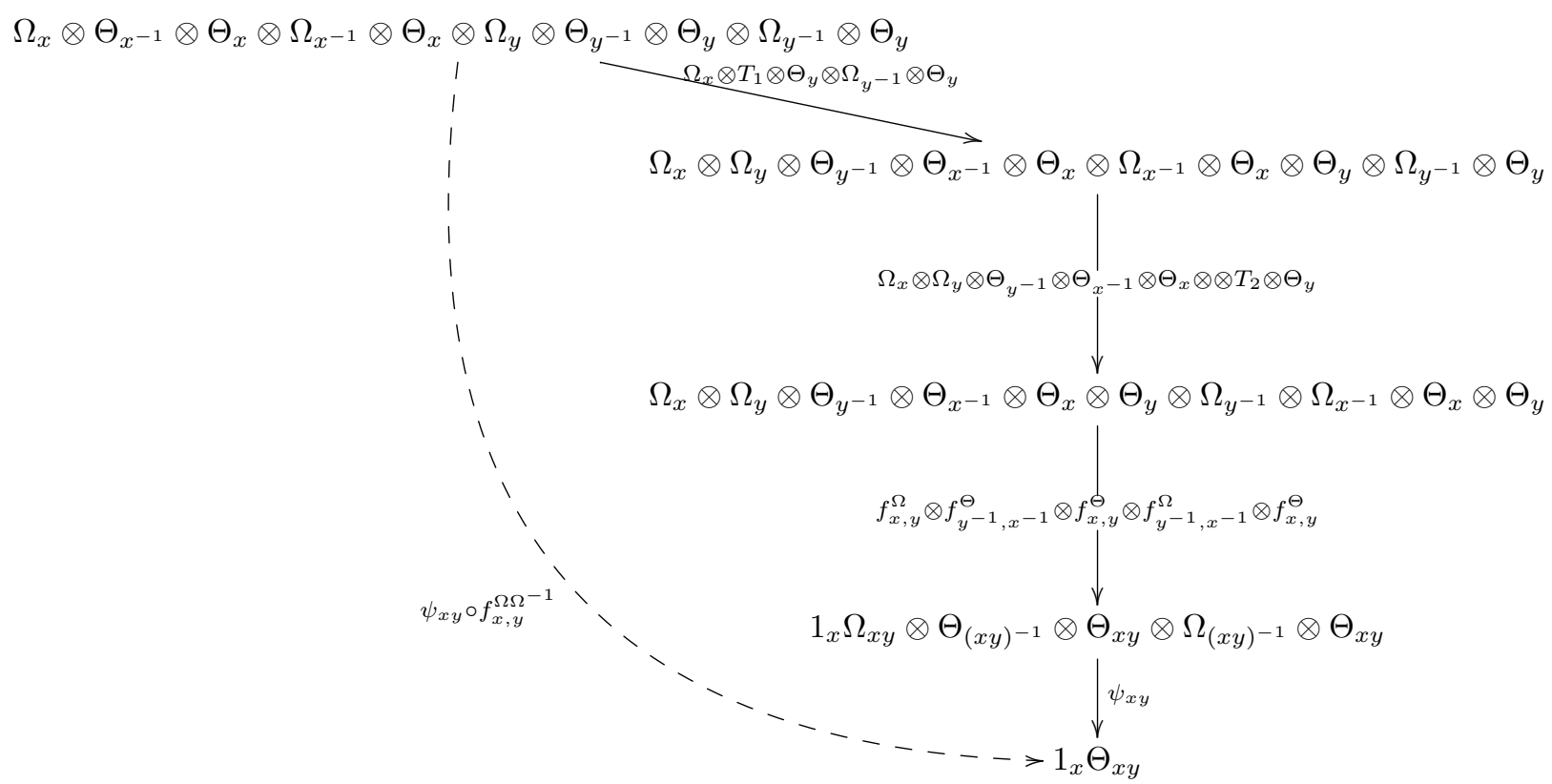

onde $T_{1}$ e $T_{2}$ são os isomorfismos:

$$
\begin{gathered}
T_{1}: \Theta_{x^{-1}} \otimes \Theta_{x} \otimes \Omega_{x^{-1}} \otimes \Theta_{x} \otimes \Omega_{y} \otimes \Theta_{y^{-1}} \longrightarrow \Omega_{y} \otimes \Theta_{y^{-1}} \otimes \Theta_{x^{-1}} \otimes \Theta_{x} \otimes \Omega_{x^{-1}} \otimes \Theta_{x} \\
T_{2}: \Omega_{x^{-1}} \otimes \Theta_{x} \otimes \Theta_{y} \otimes \Omega_{y^{-1}} \longrightarrow \Theta_{y} \otimes \Omega_{y^{-1}} \otimes \Omega_{x^{-1}} \otimes \Theta_{x}
\end{gathered}
$$

Vamos construir os isomorfismos $T_{1}$ e $T_{2}$ : Sejam $f_{i}: \Omega_{x^{-1}} \otimes \Theta_{x} \longrightarrow R$ e $g_{i}: R \longrightarrow \Omega_{x^{-1}} \otimes \Theta_{x}$, com $i=1,2, \ldots, n$, aplicações $R$-bilineares tais que $\sum_{i=1}^{n} g_{i} f_{i}=I d_{\Omega_{x-1} \otimes_{R} \Theta_{x}}$. Como $f_{i}$ e $g_{i}$ são $R$-bilineares, então $f_{j}\left(g_{i}(1)\right) \in \mathcal{Z}$, para todo $i, j=1, \ldots, n$. Vamos denotar $g_{i}(1)=\sum_{l} \omega_{x^{-1}}^{i, l} \otimes u_{x}^{i, l}$. Então,

$$
\begin{aligned}
T_{2}: \Omega_{x^{-1}} \otimes_{R} \Theta_{x} \otimes_{R} \Theta_{y} \otimes_{R} \Omega_{y^{-1}} & \longrightarrow \Theta_{y} \otimes_{R} \Omega_{y^{-1}} \otimes_{R} \Omega_{x^{-1}} \otimes_{R} \Theta_{x} \\
\omega_{x^{-1}} \otimes u_{x} \otimes u_{y} \otimes \omega_{y^{-1}} & \longmapsto \sum_{i, l} f_{i}\left(\omega_{x^{-1}} \otimes u_{x}\right) u_{y} \otimes \omega_{y^{-1}} \otimes \omega_{x^{-1}}^{i, l} \otimes u_{x}^{i, l} .
\end{aligned}
$$


Para construir $T_{2}$ vamos considerar as aplicações $R$-bilineares

$$
\begin{aligned}
& \bar{f}_{i}: \Theta_{x^{-1}} \otimes \Theta_{x} \otimes \Omega_{x^{-1}} \otimes \Theta_{x} \longrightarrow R 1_{x^{-1}} \otimes \Omega_{x^{-1}} \otimes \Theta_{x} \longrightarrow \Omega_{x^{-1}} \otimes \Theta_{x} \stackrel{f_{i}}{\longrightarrow} R, \\
& \bar{g}_{i}: R \stackrel{g_{i}}{\longrightarrow} \Omega_{x^{-1}} \otimes \Theta_{x} \longrightarrow R 1_{x^{-1}} \otimes \Omega_{x^{-1}} \otimes \Theta_{x} \longrightarrow \Theta_{x^{-1}} \otimes \Theta_{x} \otimes \Omega_{x^{-1}} \otimes \Theta_{x} .
\end{aligned}
$$

Então,

$$
\begin{gathered}
\bar{f}_{i}\left(u_{x^{-1}} \otimes u_{x} \otimes \omega_{x^{-1}} \otimes u_{x}^{\prime}\right)=f_{i}\left(\left(u_{x^{-1}} \stackrel{\ominus}{\circ} u_{x}\right) \omega_{x^{-1}} \otimes u_{x}^{\prime}\right), \\
\bar{g}_{i}(1)=\sum_{j, l} \bar{u}_{x^{-1}}^{j} \otimes \bar{u}_{x}^{j} \otimes \omega_{x^{-1}}^{i, l} \otimes u_{x}^{i, l},
\end{gathered}
$$

onde $f_{x^{-1}, x}^{\Theta}\left(\sum_{j=1}^{m} \bar{u}_{x^{-1}}^{j} \otimes \bar{u}_{x}^{j}\right)=1_{x^{-1}}$. É fácil ver que $\sum_{i=1}^{n} \bar{g}_{i} \bar{f}_{i}=I d_{\Theta_{x^{-1}} \otimes \Theta_{x} \otimes_{R} \Omega_{x^{-1}} \otimes_{R} \Theta_{x}}$. Então, podemos escrever $T_{1}$ em função de $\bar{f}_{i}$ e $\bar{g}_{i}$ da seguinte forma:

$$
\begin{aligned}
T_{1}: \quad \Theta_{x^{-1}} \otimes \Theta_{x} \otimes \Omega_{x^{-1}} \otimes \Theta_{x} \otimes \Omega_{y} \otimes \Theta_{y^{-1}} & \rightarrow \Omega_{y} \otimes \Theta_{y^{-1}} \otimes \Theta_{x^{-1}} \otimes \Theta_{x} \otimes \Omega_{x^{-1}} \otimes \Theta_{x} \\
u_{x^{-1}} \otimes u_{x} \otimes \omega_{x^{-1}} \otimes u_{x^{-1}}^{\prime} \otimes \omega_{y} \otimes u_{y^{-1}} & \mapsto \sum_{i, j, l} f_{i}\left(\left(u_{x^{-1}} \stackrel{\ominus}{\circ} u_{x}\right) \omega_{x^{-1}} \otimes u_{x}^{\prime}\right) \omega_{y} \otimes u_{y^{-1}} \otimes \bar{u}_{x^{-1}}^{j} \otimes \bar{u}_{x}^{j} \otimes \omega_{x^{-1}}^{i, l} \otimes u_{x}^{i, l} .
\end{aligned}
$$

Assim, o outro lado do diagrama é dado por:

$$
\begin{aligned}
& \omega_{x} \otimes u_{x^{-1}} \otimes u_{x} \otimes \omega_{x^{-1}} \otimes u_{x}^{\prime} \otimes \omega_{y} \otimes u_{y^{-1}} \otimes u_{y} \otimes \omega_{y^{-1}} \otimes u_{y}^{\prime} \\
& \stackrel{T_{f}}{\mapsto} \sum_{i, j} \omega_{x} \otimes f_{i}\left(\left(u_{x^{-1}} \stackrel{\ominus}{\circ} u_{x}\right) \omega_{x^{-1}} \otimes u_{x}^{\prime}\right) \omega_{y} \otimes u_{y^{-1}} \otimes \bar{u}_{x^{-1}}^{j} \otimes \bar{u}_{x}^{j} \otimes g_{i}(1) \otimes u_{y} \otimes \omega_{y^{-1}} \otimes u_{y}^{\prime} \\
& \stackrel{T_{2}}{\rightrightarrows} \sum_{i, j, k, l^{\prime}} \omega_{x} \otimes f_{i}\left(\left(u_{x^{-1}} \stackrel{\ominus}{\circ} u_{x}\right) \omega_{x^{-1}} \otimes u_{x}^{\prime}\right) \omega_{y} \otimes u_{y^{-1}} \otimes \bar{u}_{x^{-1}}^{j} \otimes \bar{u}_{x}^{j} \otimes \underbrace{f_{k}\left(g_{i}(1)\right)}_{\in \mathcal{Z}} u_{y} \otimes \omega_{y^{-1}} \otimes \omega_{x^{-1}}^{k, l^{\prime}} \otimes u_{x}^{k, l^{\prime}} \otimes u_{y}^{\prime} \\
& \stackrel{(3.15)}{=} \sum_{i, j, k, l^{\prime}} \omega_{x} \otimes f_{i}\left(\left(u_{x^{-1}} \stackrel{\ominus}{\circ} u_{x}\right) \omega_{x^{-1}} \otimes u_{x}^{\prime}\right) f_{k}\left(g_{i}(1)\right) \omega_{y} \otimes u_{y^{-1}} \otimes \bar{u}_{x^{-1}}^{j} \otimes \bar{u}_{x}^{j} \otimes u_{y} \otimes \omega_{y^{-1}} \otimes \omega_{x^{-1}}^{k, l^{\prime}} \otimes u_{x}^{k, l^{\prime}} \otimes u_{y}^{\prime} \\
& =\sum_{i, j, k, l, l^{\prime}} \omega_{x} \otimes f_{k}\left(g_{i}\left(f_{i}\left(\left(u_{x^{-1}} \stackrel{\ominus}{\circ} u_{x}\right) \omega_{x^{-1}} \otimes u_{x}^{\prime}\right)\right)\right) \omega_{y} \otimes u_{y^{-1}} \otimes \bar{u}_{x^{-1}}^{j} \otimes \bar{u}_{x}^{j} \otimes u_{y} \otimes \omega_{y^{-1}} \otimes \omega_{x^{-1}}^{k, l^{\prime}} \otimes u_{x}^{k, l^{\prime}} \otimes u_{y}^{\prime} \\
& =\sum_{j, k, l^{\prime}} \omega_{x} \otimes f_{k}\left(\left(u_{x^{-1}} \stackrel{\ominus}{\circ} u_{x}\right) \omega_{x^{-1}} \otimes u_{x}^{\prime}\right) \omega_{y} \otimes u_{y^{-1}} \otimes \bar{u}_{x^{-1}}^{j} \otimes \bar{u}_{x}^{j} \otimes u_{y} \otimes \omega_{y^{-1}} \otimes \omega_{x^{-1}}^{k, l^{\prime}} \otimes u_{x}^{k, l^{\prime}} \otimes u_{y}^{\prime} \\
& \mapsto \quad \sum_{j, k, l^{\prime}}\left(\omega_{x} \stackrel{\Omega}{\circ} f_{k}\left(\left(u_{x^{-1}} \stackrel{\ominus}{\circ} u_{x}\right) \omega_{x^{-1}} \otimes u_{x}^{\prime}\right) \omega_{y}\right) \otimes\left(u_{y^{-1}} \stackrel{\ominus}{\circ} \bar{u}_{x^{-1}}^{j}\right) \otimes\left(\bar{u}_{x}^{j} \stackrel{\stackrel{\ominus}{\circ} u_{y}}{ }\right) \otimes\left(\omega_{y^{-1}} \stackrel{\Omega}{\circ} \omega_{x^{-1}}^{k, l^{\prime}}\right) \otimes\left(u_{x}^{k, l^{\prime}} \stackrel{\ominus}{\circ} u_{y}^{\prime}\right) \\
& \stackrel{\psi_{x y}}{\mapsto} \sum_{j, k, l^{\prime}}\left(\left(\omega_{x} \stackrel{\Omega}{\circ} f_{k}\left(\left(u_{x^{-1}} \stackrel{\ominus}{\circ} u_{x}\right) \omega_{x^{-1}} \otimes u_{x}^{\prime}\right) \omega_{y}\right)\left(u_{y^{-1}} \stackrel{\ominus}{\circ} \bar{u}_{x^{-1}}^{j} \stackrel{\ominus}{\circ} \bar{u}_{x}^{j} \stackrel{\ominus}{\circ} u_{y}\right) \stackrel{\Omega}{\circ}\left(\omega_{y^{-1}} \stackrel{\Omega}{\circ} \omega_{x^{-1}}^{k, l^{\prime}}\right)\right)\left(u_{x}^{k, l^{\prime}} \stackrel{\ominus}{\circ} u_{y}^{\prime}\right) \\
& =\sum_{k, l^{\prime}}\left(\left(\omega_{x} \stackrel{\Omega}{\circ}\left(u_{x^{-1}} \stackrel{\ominus}{\circ} u_{x}\right) f_{k}\left(\omega_{x^{-1}} \otimes u_{x}^{\prime}\right) \omega_{y}\right)\left(u_{y^{-1}} 1_{x^{-1}} \stackrel{\ominus}{\circ} u_{y}\right) \stackrel{\Omega}{\circ}\left(\omega_{y^{-1}} \stackrel{\Omega}{\circ} \omega_{x^{-1}}^{k, l^{\prime}}\right)\right)\left(u_{x}^{k, l^{\prime}} \stackrel{\ominus}{\circ} u_{y}^{\prime}\right)
\end{aligned}
$$

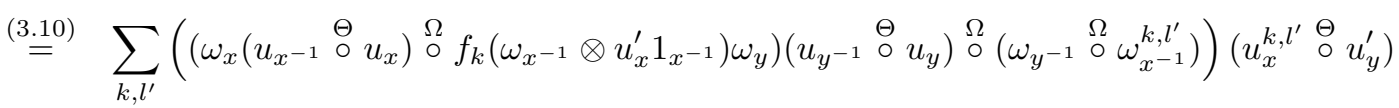




$$
\begin{aligned}
& =\sum_{k, l^{\prime}}\left(\left(\omega_{x}\left(u_{x^{-1}} \stackrel{\ominus}{\circ} u_{x}\right) \stackrel{\Omega}{\circ} f_{k}\left(\omega_{x^{-1}} \otimes u_{x}^{\prime}\right) \omega_{y}\right)\left(u_{y^{-1}} \stackrel{\ominus}{\circ} u_{y}\right) \stackrel{\Omega}{\circ}\left(\omega_{y^{-1}} \stackrel{\Omega}{\circ} \omega_{x^{-1}}^{k, l^{\prime}}\right)\right)\left(u_{x}^{k, l^{\prime}} \stackrel{\ominus}{\circ} u_{y}^{\prime}\right) \\
& =\sum_{k, l^{\prime}}\left(\omega_{x}\left(u_{x^{-1}} \stackrel{\ominus}{\circ} u_{x}\right) \stackrel{\Omega}{\circ}\left(f_{k}\left(\omega_{x^{-1}} \otimes u_{x}^{\prime}\right) \omega_{y}\left(u_{y^{-1}} \stackrel{\ominus}{\circ} u_{y}\right) \stackrel{\Omega}{\circ} \omega_{y^{-1}}\right) \stackrel{\Omega}{\circ} \omega_{x^{-1}}^{k, l^{\prime}}\right)\left(u_{x}^{k, l^{\prime}} \stackrel{\ominus}{\circ} u_{y}^{\prime}\right)
\end{aligned}
$$

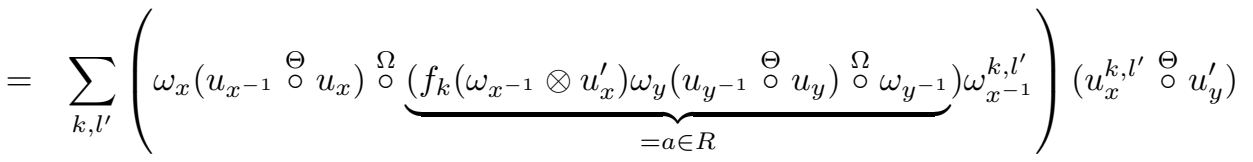

$$
\begin{aligned}
& =\sum_{k, l^{\prime}}\left(\left(\omega_{x}\left(u_{x-1} \stackrel{\ominus}{\circ} u_{x}\right) \stackrel{\Omega}{\circ} a \omega_{x^{-1}}^{k, l^{\prime}}\right) u_{x}^{k, l^{\prime}} \stackrel{\ominus}{\circ} u_{y}^{\prime}\right) \\
& =\sum_{k, l^{\prime}}\left(f_{x, y}^{\Theta} \circ\left(f_{x, x^{-1}}^{\Omega} \otimes \Theta_{x} \otimes \Theta_{y}\right)\right)(\omega_{x}\left(u_{x^{-1}} \stackrel{\ominus}{\circ} u_{x}\right) \otimes a \underbrace{\omega_{x-1}^{k, l^{\prime}} \otimes u_{x}^{k, l^{\prime}}}_{\in C_{\Omega_{x}-1} \otimes \Theta_{x}(R)} \otimes u_{y}^{\prime}) \\
& =\sum_{k, l^{\prime}}\left(f_{x, y}^{\Theta} \circ\left(f_{x, x^{-1}}^{\Omega} \otimes \Theta_{x} \otimes \Theta_{y}\right)\right)\left(\omega_{x}\left(u_{x^{-1}} \stackrel{\ominus}{\circ} u_{x}\right) \otimes \omega_{x^{-1}}^{k, l^{\prime}} \otimes u_{x}^{k, l^{\prime}} a \otimes u_{y}^{\prime}\right) \\
& =\sum_{k, l^{\prime}}\left(f_{x, y}^{\Theta} \circ\left(f_{x, x^{-1}}^{\Omega} \otimes \Theta_{x} \otimes \Theta_{y}\right)\right)\left(\omega_{x}\left(u_{x^{-1}} \stackrel{\ominus}{\circ} u_{x}\right) \otimes \omega_{x^{-1}}^{k, l^{\prime}} \otimes u_{x}^{k, l^{\prime}} f_{k}\left(\omega_{x^{-1}} \otimes u_{x}^{\prime}\right)\left(\omega_{y}\left(u_{y^{-1}} \stackrel{\ominus}{\circ} u_{y}\right) \stackrel{\Omega}{\circ} \omega_{y^{-1}}\right) \otimes u_{y}^{\prime}\right) \\
& =\sum_{k}\left(f_{x, y}^{\Theta} \circ\left(f_{x, x^{-1}}^{\Omega} \otimes \Theta_{x} \otimes \Theta_{y}\right)\right)\left(\omega_{x}\left(u_{x^{-1}} \stackrel{\ominus}{\circ} u_{x}\right) \otimes g_{k}(1) f_{k}\left(\omega_{x^{-1}} \otimes u_{x}^{\prime}\right) \otimes\left(\omega_{y}\left(u_{y^{-1}} \stackrel{\ominus}{\circ} u_{y}\right) \stackrel{\Omega}{\circ} \omega_{y^{-1}}\right) u_{y}^{\prime}\right) \\
& =\sum_{k}\left(f_{x, y}^{\Theta} \circ\left(f_{x, x^{-1}}^{\Omega} \otimes \Theta_{x} \otimes \Theta_{y}\right)\right)\left(\omega_{x}\left(u_{x^{-1}} \stackrel{\ominus}{\circ} u_{x}\right) \otimes g_{k}\left(f_{k}\left(\omega_{x^{-1}} \otimes u_{x}^{\prime}\right)\right) \otimes\left(\omega_{y}\left(u_{y^{-1}} \stackrel{\ominus}{\circ} u_{y}\right) \stackrel{\Omega}{\circ} \omega_{y^{-1}}\right) u_{y}^{\prime}\right) \\
& =\left(f_{x, y}^{\Theta} \circ\left(f_{x, y}^{\Omega} \otimes \Theta_{x} \otimes \Theta_{y}\right)\right)\left(\omega_{x}\left(u_{x^{-1}} \stackrel{\ominus}{\circ} u_{x}\right) \otimes \omega_{x^{-1}} \otimes u_{x}^{\prime} \otimes\left(\omega_{y}\left(u_{y^{-1}} \stackrel{\ominus}{\circ} u_{y}\right) \stackrel{\Omega}{\circ} \omega_{y^{-1}}\right) u_{y}^{\prime}\right)
\end{aligned}
$$

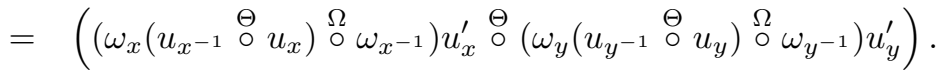

Segue então de (3.35) e (3.36) que o diagrama é comutativo. Portanto, $[\Delta(\Omega)][\Delta(\Omega)]^{-1}=[\Delta(\Theta)]$

Comutatividade: Sejam $[\Delta(\Gamma)],[\Delta(\Omega)] \in \mathcal{C}(\Theta / R)$. Temos

$$
[\Delta(\Gamma)][\Delta(\Omega)]=\left[\bigoplus_{x \in G} \Gamma_{x} \otimes_{R} \Theta_{x^{-1}} \otimes_{R} \Omega_{x}\right] \text { e }[\Delta(\Omega)][\Delta(\Gamma)]=\left[\bigoplus_{x \in G} \Omega_{x} \otimes_{R} \Theta_{x^{-1}} \otimes_{R} \Gamma_{x}\right] .
$$

Para cada $x \in G$, considere o isomorfismo $\lambda_{x}$ definido por

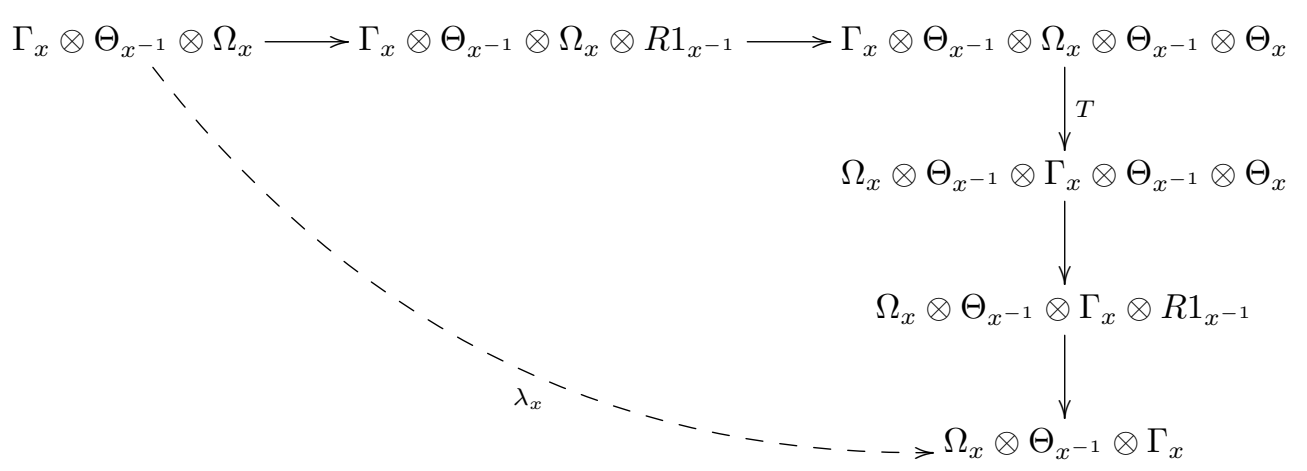

ou seja,

$$
\lambda_{x}=\left(\Omega_{x} \otimes \Theta_{x^{-1}} \otimes \Gamma_{x} \otimes f_{x^{-1}, x}^{\Theta}\right) \circ\left(T \otimes \Theta_{x}\right) \circ\left(\Gamma_{x} \otimes \Theta_{x^{-1}} \otimes \Omega_{x} \otimes\left(f_{x^{-1}, x}^{\Theta}\right)^{-1}\right),
$$

onde $T: \Gamma_{x} \otimes \Theta_{x^{-1}} \otimes \Omega_{x} \otimes \Theta_{x^{-1}} \longrightarrow \Omega_{x} \otimes \Theta_{x^{-1}} \otimes \Gamma_{x} \otimes \Theta_{x^{-1}}$ é o isomorfismo dado na Proposição 2.3.5. 
Devemos mostrar que o diagrama abaixo é comutativo

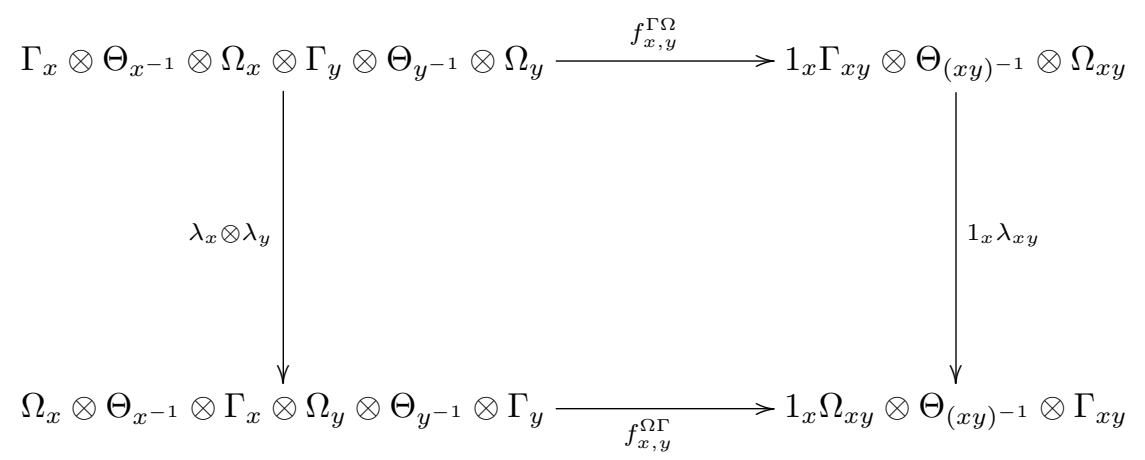

Sejam

$$
\begin{gathered}
T_{1}: \Gamma_{x} \otimes \Theta_{x^{-1}} \otimes \Omega_{x} \otimes \Theta_{x^{-1}} \longrightarrow \Omega_{x} \otimes \Theta_{x^{-1}} \otimes \Gamma_{x} \otimes \Theta_{x^{-1}} \\
T_{2}: \Gamma_{y} \otimes \Theta_{y^{-1}} \otimes \Omega_{y} \otimes \Theta_{y^{-1}} \longrightarrow \Omega_{y} \otimes \Theta_{y^{-1}} \otimes \Gamma_{y} \otimes \Theta_{y^{-1}} \\
T_{3}: \Theta_{x^{-1}} \otimes \Gamma_{x} \otimes \Omega_{y} \otimes \Theta_{y^{-1}} \longrightarrow \Omega_{y} \otimes \Theta_{y^{-1}} \otimes \Theta_{x^{-1}} \otimes \Gamma_{x} \\
T_{4}: \Theta_{x^{-1}} \otimes \Omega_{x} \otimes \Gamma_{y} \otimes \Theta_{y^{-1}} \rightarrow \Gamma_{y} \otimes \Theta_{y^{-1}} \otimes \Theta_{x^{-1}} \otimes \Omega_{x} \\
T_{5}: \Gamma_{x y} \otimes \Theta_{(x y)^{-1}} \otimes 1_{x} \Omega_{x y} \otimes 1_{y^{-1}} \Theta_{(x y)^{-1}} \rightarrow 1_{x} \Omega_{x y} \otimes 1_{y^{-1}} \Theta_{(x y)^{-1}} \otimes \Gamma_{x y} \otimes \Theta_{(x y)^{-1}}
\end{gathered}
$$

isomorfismos como na Proposição 2.3.5, então $f_{x, y}^{\Omega \Lambda} \circ\left(\lambda_{x} \otimes \lambda_{y}\right)$ é dado por:

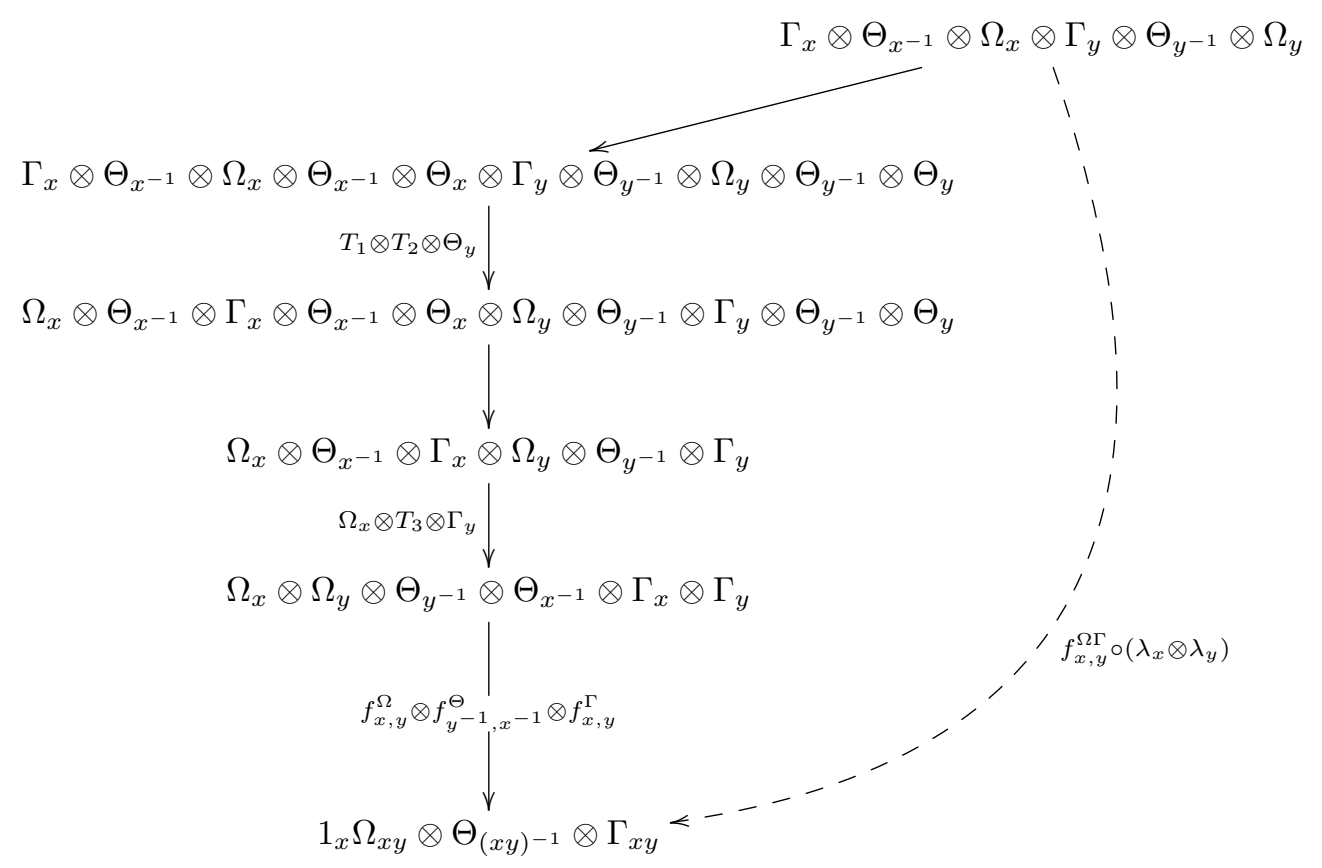

Por outro lado, $1_{x} \lambda_{x y} \circ f_{x, y}^{\Gamma \Omega}$ é dado por: 


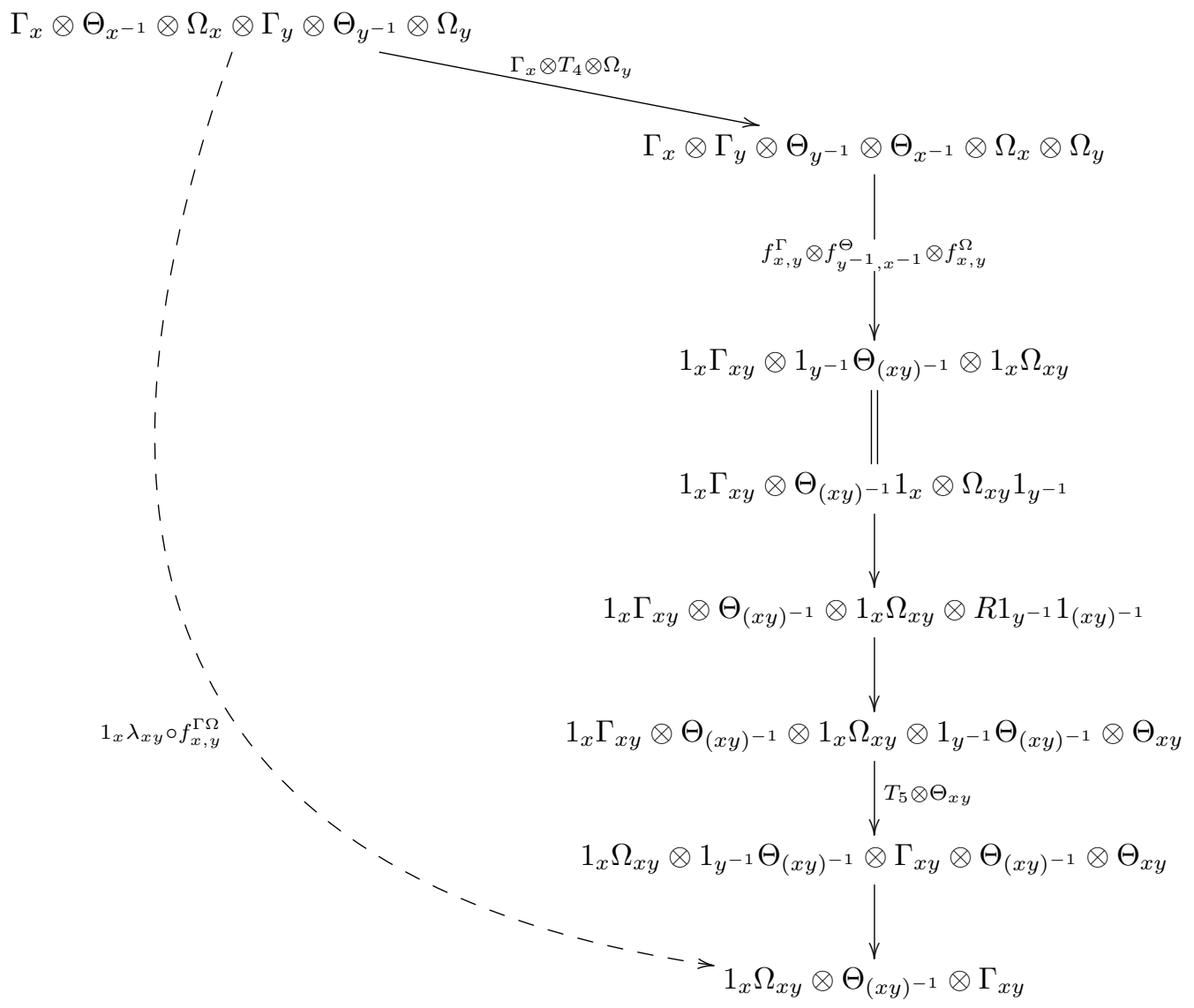

Vamos construir os isomorfismos $T_{i}$ 's: Para os isomorfismos $T_{2}$ e $T_{4}$ considere as aplicações $R$-bilineares $f_{l}: \Gamma_{y} \otimes_{R} \Omega_{y^{-1}} \longrightarrow R$ e $g_{l}: R \longrightarrow \Gamma_{y} \otimes_{R} \Omega_{y^{-1}}, \operatorname{com} l=1,2, \ldots, k$, tais que $\sum_{l=1}^{k} g_{l} f_{l}=I d_{\Gamma_{y} \otimes_{R} \Omega_{y^{-1}}}$. Denote $g_{l}(1)=\sum_{l, t} v_{y}^{l, t} \otimes u_{y^{-1}}^{l, t}$. Então,

$$
\begin{aligned}
T_{2}: \quad \Gamma_{y} \otimes_{R} \Theta_{y^{-1}} \otimes_{R} \Omega_{y} \otimes_{R} \Theta_{y^{-1}} & \rightarrow \Omega_{y} \otimes_{R} \Theta_{y^{-1}} \otimes_{R} \Gamma_{y} \otimes_{R} \Theta_{y^{-1}} \\
v_{y} \otimes u_{y^{-1}} \otimes \omega_{y} \otimes u_{y^{-1}}^{\prime} & \mapsto \sum_{l, t} f_{l}\left(v_{y} \otimes u_{y^{-1}}\right) \omega_{y} \otimes u_{y^{-1}}^{\prime} \otimes v_{y}^{l, t} \otimes u_{y^{-1}}^{l, t} \\
T_{4}: \quad \Theta_{x^{-1}} \otimes_{R} \Omega_{x} \otimes_{R} \Gamma_{y} \otimes_{R} \Theta_{y^{-1}} & \rightarrow \Gamma_{y} \otimes_{R} \Theta_{y^{-1}} \otimes_{R} \Theta_{x^{-1}} \otimes_{R} \Omega_{x} \\
u_{x^{-1}} \otimes \omega_{x} \otimes v_{y} \otimes u_{y^{-1}} & \mapsto \sum_{l, t}^{l, t} v_{y}^{l, t} \otimes u_{y^{-1}}^{l, t} \otimes u_{x^{-1}} \otimes \omega_{x} f_{l}\left(v_{y} \otimes u_{y^{-1}}\right)
\end{aligned}
$$

Para $T_{1}$ e $T_{3}$ considere as aplicações $R$-bilineares $f_{i}^{x}: \Omega_{x} \otimes_{R} \Theta_{x^{-1}} \longrightarrow R, g_{i}^{x}: R \longrightarrow \Omega_{x} \otimes_{R} \Theta_{x^{-1}}$, $f_{j}^{y}: \Omega_{y} \otimes_{R} \Theta_{y^{-1}} \longrightarrow R$ e $f_{j}^{y}: R \longrightarrow \Omega_{y} \otimes_{R} \Theta_{y^{-1}}$, com $i=1,2, \ldots, n$ e $j=1,2, \ldots, m$, tais que $\sum_{i=1}^{n} g_{i}^{x} f_{i}^{x}=I d_{\Omega_{x} \otimes_{R} \Theta_{x^{-1}}}$ e $\sum_{j=1}^{m} g_{j}^{y} f_{j}^{y}=I d_{\Omega_{y} \otimes_{R} \Theta_{y^{-1}}}$. Denote,

$$
g_{i}^{x}(1)=\sum_{i, p} \widetilde{\omega}_{x}^{i, p} \otimes \widetilde{u}_{x^{-1}}^{i, p} \text { e } g_{j}^{y}(1)=\sum_{j, q} \widehat{\omega}_{y}^{j, q} \otimes \widehat{u}_{y^{-1}}^{j, q} .
$$


Então,

$$
\begin{aligned}
T_{1}: \quad \Gamma_{x} \otimes_{R} \Theta_{x^{-1}} \otimes_{R} \Omega_{x} \otimes_{R} \Theta_{x^{-1}} & \rightarrow \Omega_{x} \otimes_{R} \Theta_{x^{-1}} \otimes_{R} \Gamma_{x} \otimes_{R} \Theta_{x^{-1}} \\
v_{x} \otimes u_{x^{-1}} \otimes \omega_{x} \otimes u_{x^{-1}}^{\prime} & \mapsto \sum_{i, p} \widetilde{\omega}_{x}^{i, p} \otimes \widetilde{u}_{x^{-1}}^{i, p} \otimes v_{x} \otimes u_{x^{-1}} f_{i}^{x}\left(\omega_{x} \otimes u_{x^{-1}}^{\prime}\right) . \\
T_{3}: \quad \Theta_{x^{-1}} \otimes_{R} \Gamma_{x} \otimes_{R} \Omega_{y} \otimes_{R} \Theta_{y^{-1}} & \rightarrow \Omega_{y} \otimes_{R} \Theta_{y^{-1}} \otimes_{R} \Theta_{x^{-1}} \otimes_{R} \Gamma_{x} \\
u_{x^{-1}} \otimes v_{x} \otimes \omega_{y} \otimes u_{y^{-1}} & \mapsto \sum_{j, q} \widehat{\omega}_{y}^{j, q} \otimes \widehat{u}_{y^{-1}}^{j, q} \otimes u_{x^{-1}} \otimes v_{x} f_{j}^{y}\left(\omega_{y} \otimes u_{y^{-1}}^{\prime}\right) .
\end{aligned}
$$

Para construir $T_{5}$ considere

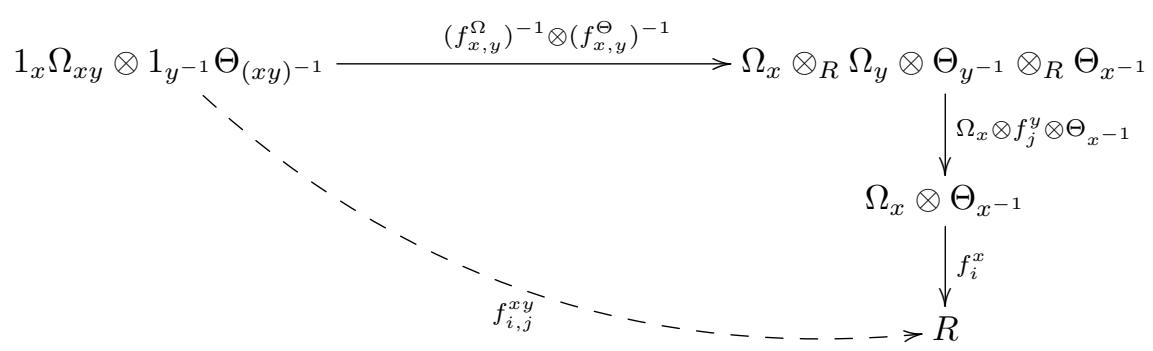

Então, $f_{i, j}^{x y}$ é $R$-bilinear e

$$
f_{i, j}^{x y}\left(\omega_{x y} \otimes u_{(x y)^{-1}}\right)=\sum f_{i}^{x}\left(\omega_{x}^{g} f_{j}^{y}\left(\omega_{y}^{g} \otimes u_{y^{-1}}^{h}\right) \otimes u_{x^{-1}}^{h}\right)
$$

onde $\sum_{g}\left(\omega_{x}^{g} \stackrel{\Omega}{\circ} \omega_{y}^{g}\right)=\omega_{x y}$ e $\sum_{h}\left(u_{y^{-1}}^{h} \stackrel{\odot}{\circ} u_{x^{-1}}^{h}\right)=u_{(x y)^{-1}}$. Analogamente, considere

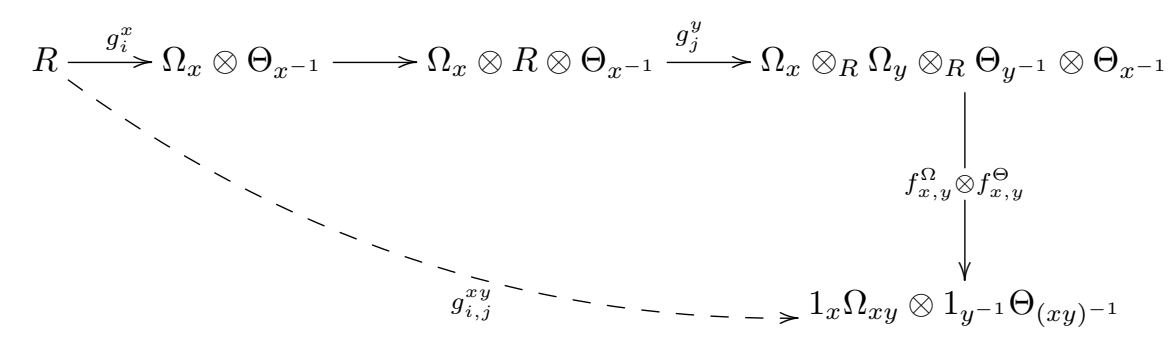

Então, $g_{i, j}^{x y}$ é $R$-bilinear e

$$
g_{i, j}^{x y}(1)=\sum_{i, j, p, q}\left(\widetilde{\omega}_{x}^{i, p} \stackrel{\Omega}{\circ} \widehat{\omega}_{y}^{j, q}\right) \otimes\left(\widehat{u}_{y^{-1}}^{j q} \stackrel{\ominus}{\circ} \widetilde{u}_{x^{-1}}^{i, p}\right)
$$

É fácil ver que $\sum_{i, j}^{n, m} g_{i, j}^{x y} f_{i, j}^{x y}=I d_{1_{x} \Omega_{x y} \otimes 1_{y^{-1}} \Theta_{(x y)-1}}$. Então,

$T_{2}: \quad \Gamma_{x y} \otimes \Theta_{(x y)^{-1}} \otimes 1_{x} \Omega_{x y} \otimes 1_{y^{-1}} \Theta_{(x y)^{-1}} \quad \rightarrow \quad 1_{x} \Omega_{x y} \otimes 1_{y^{-1}} \Theta_{(x y)^{-1}} \otimes \Gamma_{x y} \otimes \Theta_{(x y)^{-1}}$ $v_{x y} \otimes u_{(x y)^{-1}} \otimes \omega_{x y} \otimes u_{(x y)^{-1}}^{\prime} \mapsto \sum g_{i, j}^{x y}(1) \otimes v_{x y} \otimes u_{(x y)^{-1}} f_{i, j}^{x y}\left(\omega_{x y} \otimes u_{(x y)^{-1}}^{\prime}\right)$.

Vamos denotar

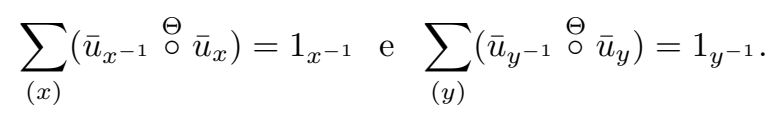


Vamos agora mostrar que o diagrama é comutativo. Para $1_{x} \lambda_{x y} \circ f_{y, x}^{\Gamma \Omega}$, usando a Observação 3.3.10 temos:

$$
\begin{aligned}
& v_{x} \otimes u_{x^{-1}} \otimes \omega_{x} \otimes v_{y} \otimes u_{y^{-1}} \otimes \omega_{y} \\
& \stackrel{T_{4}}{\mapsto} \sum_{l, t} v_{x} \otimes v_{y}^{l, t} \otimes u_{y^{-1}}^{l, t} \otimes u_{x^{-1}} \otimes \omega_{x} f_{l}\left(v_{y} \otimes u_{y^{-1}}\right) \otimes \omega_{y} \\
& \mapsto \sum_{l, t}\left(v_{x} \stackrel{\Gamma}{\circ} v_{y}^{l, t}\right) \otimes\left(u_{y^{-1}}^{l, t} \stackrel{\ominus}{\circ} u_{x^{-1}}\right) \otimes\left(\omega_{x} f_{l}\left(v_{y} \otimes u_{y^{-1}}\right) \stackrel{\Omega}{\circ} \omega_{y}\right) \\
& \mapsto \sum_{l, t}\left(v_{x} \stackrel{\Gamma}{\circ} v_{y}^{l, t}\right) \otimes\left(u_{y^{-1}}^{l, t} \stackrel{\ominus}{\circ} u_{x^{-1}}\right) \otimes\left(\omega_{x} f_{l}\left(v_{y} \otimes u_{y^{-1}}\right) \stackrel{\Omega}{\circ} \omega_{y}\right) \otimes 1_{y^{-1}} 1_{(x y)^{-1}} \\
& \mapsto \sum_{l, t}\left(v_{x} \stackrel{\Gamma}{\circ} v_{y}^{l, t}\right) \otimes\left(u_{y^{-1}}^{l, t} \stackrel{\ominus}{\circ} u_{x^{-1}}\right) \otimes\left(\omega_{x} f_{l}\left(v_{y} \otimes u_{y^{-1}}\right) \stackrel{\Omega}{\circ} \omega_{y}\right) \otimes\left(\bar{u}_{y^{-1}} \stackrel{\ominus}{\circ} \bar{u}_{x^{-1}}\right) \otimes\left(\bar{u}_{x} \stackrel{\ominus}{\circ} \bar{u}_{y}\right) \\
& \left.\stackrel{T_{5}}{\mapsto} \sum\left(\widetilde{\omega}_{x}^{i, p} \stackrel{\Omega}{\circ} \widehat{\omega}_{y}^{j, q}\right) \otimes\left(\widehat{u}_{y^{-1}}^{j, q} \stackrel{\ominus}{\circ} \widetilde{u}_{x^{-1}}^{i, p}\right) \otimes\left(v_{x} \stackrel{\Gamma}{\circ} v_{y}^{l, t}\right) \otimes\left(u_{y^{-1}}^{l, t} \stackrel{\ominus}{\circ} u_{x^{-1}}\right) f_{i}^{x}(\omega_{x} \underbrace{f_{l}\left(v_{y} \otimes u_{y^{-1}}\right) f_{j}^{y}\left(\omega_{y} \otimes \bar{u}_{y^{-1}}\right.}_{=b_{l, j} \in R}) \otimes \bar{u}_{x^{-1}}\right) \\
& \otimes\left(\bar{u}_{x} \stackrel{\ominus}{\circ} \bar{u}_{y}\right) \\
& =\sum\left(\widetilde{\omega}_{x}^{i, p} \stackrel{\Omega}{\circ} \widehat{\omega}_{y}^{j, q}\right) \otimes\left(\widehat{u}_{y^{-1}}^{j, q} \stackrel{\ominus}{\circ} \widetilde{u}_{x^{-1}}^{i, p}\right) \otimes\left(v_{x} \stackrel{\Gamma}{\circ} v_{y}^{l, t}\right) \otimes\left(u_{y^{-1}}^{l, t} \stackrel{\ominus}{\circ} u_{x^{-1}}\right) f_{i}^{x}\left(\omega_{x} b_{l, j} \otimes \bar{u}_{x^{-1}}\right) \otimes\left(\bar{u}_{x} \stackrel{\ominus}{\circ} \bar{u}_{y}\right) \\
& =\sum\left(\widetilde{\omega}_{x}^{i, p} \stackrel{\Omega}{\circ} \widehat{\omega}_{y}^{j, q}\right) \otimes\left(\widehat{u}_{y^{-1}}^{j, q} \stackrel{\ominus}{\circ} \widetilde{u}_{x^{-1}}^{i, p}\right) \otimes\left(v_{x} \stackrel{\Gamma}{\circ} v_{y}^{l, t}\right) \otimes\left(u_{y^{-1}}^{l, t} \stackrel{\ominus}{\circ} u_{x^{-1}} f_{i}^{x}\left(\omega_{x} b_{l, j} \otimes \bar{u}_{x^{-1}}\right)\right) \otimes\left(\bar{u}_{x} \stackrel{\ominus}{\circ} \bar{u}_{y}\right) \\
& \mapsto \sum\left(\widetilde{\omega}_{x}^{i, p} \stackrel{\Omega}{\circ} \widehat{\omega}_{y}^{j, q}\right) \otimes\left(\widehat{u}_{y^{-1}}^{j, q} \stackrel{\ominus}{\circ} \widetilde{u}_{x^{-1}}^{i, p}\right) \otimes\left(v_{x} \stackrel{\Gamma}{\circ} v_{y}^{l, t}\right)\left(\left(u_{y^{-1}}^{l, t} \stackrel{\ominus}{\circ} u_{x^{-1}} f_{i}^{x}\left(\omega_{x} b_{l, j} \otimes \bar{u}_{x^{-1}}\right)\right) \stackrel{\ominus}{\circ}\left(\bar{u}_{x} \stackrel{\ominus}{\circ} \bar{u}_{y}\right)\right) \\
& =\sum\left(\widetilde{\omega}_{x}^{i, p} \stackrel{\Omega}{\circ} \widehat{\omega}_{y}^{j, q}\right) \otimes\left(\widehat{u}_{y^{-1}}^{j, q} \stackrel{\ominus}{\circ} \widetilde{u}_{x^{-1}}^{i, p}\right) \otimes\left(v_{x} \stackrel{\Gamma}{\circ} v_{y}^{l, t}\right)(u_{y^{-1}}^{l, t} \stackrel{\ominus}{\circ} \underbrace{\left(u_{x^{-1} f_{i}^{x}\left(\omega_{x} b_{l, j} \otimes \bar{u}_{x^{-1}}\right) \stackrel{\ominus}{\circ}} \bar{u}_{x}\right)}_{=c_{i, j, l} \in R} \bar{u}_{y}) \\
& =\sum\left(\widetilde{\omega}_{x}^{i, p} \stackrel{\Omega}{\circ} \widehat{\omega}_{y}^{j, q}\right) \otimes\left(\widehat{u}_{y^{-1}}^{j, q} \stackrel{\ominus}{\circ} \widetilde{u}_{x^{-1}}^{i, p}\right) \otimes\left(v_{x} \stackrel{\Gamma}{\circ} v_{y}^{l, t}\right)\left(u_{y^{-1}}^{l, t} \stackrel{\ominus}{\circ} c_{i, j, l} \bar{u}_{y}\right) \\
& =\sum\left(\widetilde{\omega}_{x}^{i, p} \stackrel{\Omega}{\circ} \widehat{\omega}_{y}^{j, q}\right) \otimes\left(\widehat{u}_{y^{-1}}^{j, q} \stackrel{\ominus}{\circ} \widetilde{u}_{x^{-1}}^{i, p}\right) \otimes \underbrace{\left(v_{x} \stackrel{\Gamma}{\circ} v_{y}^{l, t}\left(u_{y^{-1}}^{l, t} \stackrel{\ominus}{\circ} c_{i, j, l} \bar{u}_{y}\right)\right)}_{(*)}
\end{aligned}
$$

Vejamos que $(*)$ pode ser escrito da seguinte forma:

$$
\begin{aligned}
\left(v_{x} \circ v_{y}^{l, t}\left(u_{y^{-1}}^{l, t} \stackrel{\ominus}{\circ} c_{i, j, l} \bar{u}_{y}\right)\right) & =\left(f_{x, y}^{\Gamma} \circ\left(\Gamma_{x} \otimes \Gamma_{y} \otimes f_{y^{-1}, y}^{\Theta}\right)\right)(v_{x} \otimes \underbrace{v_{y}^{l, t} \otimes u_{y^{-1}}^{l, t}}_{\in C_{\Gamma_{y} \otimes \Theta_{y-1}}(R)} c_{i, j, l} \otimes \bar{u}_{y}) \\
& =\left(f_{x, y}^{\Gamma} \circ\left(\Gamma_{x} \otimes \Gamma_{y} \otimes f_{y^{-1}, y}^{\Theta}\right)\right)\left(v_{x} \otimes c_{i, j, l} v_{y}^{l, t} \otimes u_{y^{-1}}^{l, t} \otimes \bar{u}_{y}\right) \\
& =\left(v_{x} \circ c_{i, j, l} v_{y}^{l, t}\left(u_{y^{-1}}^{l, t} \stackrel{\ominus}{\circ} \bar{u}_{y}\right)\right) .
\end{aligned}
$$

Voltando, temos:

$$
\begin{aligned}
& \left(1_{x} \lambda_{x y} \circ f_{y, x}^{\Gamma \Omega}\right)\left(v_{x} \otimes u_{x^{-1}} \otimes \omega_{x} \otimes v_{y} \otimes u_{y^{-1}} \otimes \omega_{y}\right) \\
= & \sum\left(\widetilde{\omega}_{x}^{i, p} \stackrel{\Omega}{\circ} \widehat{\omega}_{y}^{j, q}\right) \otimes\left(\widehat{u}_{y^{-1}}^{j, q} \stackrel{\ominus}{\circ} \widetilde{u}_{x^{-1}}^{i, p}\right) \otimes\left(v_{x} \stackrel{\Gamma}{\circ} c_{i, j, l} v_{y}^{l, t}\left(u_{y^{-1}}^{l, t} \stackrel{\ominus}{\circ} \bar{u}_{y}\right)\right) \\
= & \sum\left(\widetilde{\omega}_{x}^{i, p} \stackrel{\Omega}{\circ} \widehat{\omega}_{y}^{j, q}\right) \otimes\left(\widehat{u}_{y^{-1}}^{j, q} \stackrel{\ominus}{\circ} \widetilde{u}_{x^{-1}}^{i, p}\right) \otimes\left(v_{x}\left(u_{x^{-1}} f_{i}^{x}\left(\omega_{x} b_{l, j} \otimes \bar{u}_{x^{-1}}\right) \stackrel{\ominus}{\circ} \bar{u}_{x}\right) \stackrel{\Gamma}{\circ} v_{y}^{l, t}\left(u_{y^{-1}}^{l, t} \stackrel{\ominus}{\circ} \bar{u}_{y}\right)\right)
\end{aligned}
$$


Por fim, vamos calcular $f_{x, y}^{\Omega \Gamma} \circ\left(\lambda_{x} \otimes \lambda_{y}\right)$ :

$$
\begin{aligned}
& v_{x} \otimes u_{x^{-1}} \otimes \omega_{x} \otimes v_{y} \otimes u_{y^{-1}} \otimes \omega_{y} \\
& \mapsto \quad v_{x} \otimes u_{x^{-1}} \otimes \omega_{x} \otimes 1_{x^{-1}} \otimes v_{y} \otimes u_{y^{-1}} \otimes \omega_{y} \otimes 1_{y^{-1}} \\
& \mapsto \quad \sum v_{x} \otimes u_{x^{-1}} \otimes \omega_{x} \otimes \bar{u}_{x^{-1}} \otimes \bar{u}_{x} \otimes v_{y} \otimes u_{y^{-1}} \otimes \omega_{y} \otimes \bar{u}_{y^{-1}} \otimes \bar{u}_{y} \\
& \stackrel{T_{1}, T_{2}}{\mapsto} \sum \widetilde{\omega}_{x}^{i, p} \otimes \widetilde{u}_{x^{-1}}^{i, p} \otimes v_{x} \otimes u_{x^{-1}} f_{i}^{x}\left(\omega_{x} \otimes \bar{u}_{x^{-1}}\right) \otimes \bar{u}_{x} \otimes f_{l}\left(v_{y} \otimes u_{y^{-1}}\right) \omega_{y} \otimes \bar{u}_{y^{-1}} \otimes v_{y}^{l, t} \otimes u_{y^{-1}}^{l, t} \otimes \bar{u}_{y} \\
& \mapsto \quad \sum \widetilde{\omega}_{x}^{i, p} \otimes \widetilde{u}_{x^{-1}}^{i, p} \otimes v_{x}\left(u_{x^{-1}} f_{i}^{x}\left(\omega_{x} \otimes \bar{u}_{x^{-1}}\right) \stackrel{\ominus}{\circ} \bar{u}_{x}\right) \otimes f_{l}\left(v_{y} \otimes u_{y^{-1}}\right) \omega_{y} \otimes \bar{u}_{y^{-1}} \otimes v_{y}^{l, t}\left(u_{y^{-1}}^{l, t} \stackrel{\ominus}{\circ} \bar{u}_{y}\right) \\
& \stackrel{T_{3}}{\mapsto} \sum \widetilde{\omega}_{x}^{i, p} \otimes \widehat{\omega}_{y}^{j, q} \otimes \widehat{u}_{y^{-1}}^{j, q} \otimes \widetilde{u}_{x^{-1}}^{i, p} \otimes v_{x}\left(u_{x^{-1}} f_{i}^{x}\left(\omega_{x} \otimes \bar{u}_{x^{-1}}\right) \stackrel{\ominus}{\circ} \bar{u}_{x}\right) f_{j}^{y}\left(f_{l}\left(v_{y} \otimes u_{y^{-1}}\right) \omega_{y} \otimes \bar{u}_{y^{-1}}\right) \otimes v_{y}^{l, t}\left(u_{y^{-1}}^{l, t} \stackrel{\ominus}{\circ} \bar{u}_{y}\right) \\
& =\sum \widetilde{\omega}_{x}^{i, p} \otimes \widehat{\omega}_{y}^{j, q} \otimes \widehat{u}_{y^{-1}}^{j, q} \otimes \widetilde{u}_{x^{-1}}^{i, p} \otimes v_{x}\left(u_{x^{-1}} f_{i}^{x}\left(\omega_{x} \otimes \bar{u}_{x^{-1}}\right) \stackrel{\ominus}{\circ} \bar{u}_{x}\right) b_{l, j} \otimes v_{y}^{l, t}\left(u_{y^{-1}}^{l, t} \stackrel{\ominus}{\circ} \bar{u}_{y}\right) \\
& \mapsto \quad \sum\left(\widetilde{\omega}_{x}^{i, p} \stackrel{\Omega}{\circ} \widehat{\omega}_{y}^{j, q}\right) \otimes\left(\widehat{u}_{y^{-1}}^{j, q} \stackrel{\ominus}{\circ} \widetilde{u}_{x^{-1}}^{i, p}\right) \otimes(v_{x} \underbrace{\left(u_{x^{-1}} f_{i}^{x}\left(\omega_{x} \otimes \bar{u}_{x^{-1}}\right) \stackrel{\ominus}{\circ} \bar{u}_{x}\right) b_{l, j}}_{(* *)} \stackrel{\Gamma}{\circ} v_{y}^{l, t}\left(u_{y^{-1}}^{l, t} \stackrel{\ominus}{\circ} \bar{u}_{y}\right)) \text {. }
\end{aligned}
$$

Vejamos que $(* *)$ pode ser escrito da seguinte forma:

$$
\begin{aligned}
\left(u_{x^{-1}} f_{i}^{x}\left(\omega_{x} \otimes \bar{u}_{x^{-1}}\right) \stackrel{\ominus}{\circ} \bar{u}_{x} b_{l j}\right) & =\left(f_{x^{-1}, x}^{\Theta} \circ\left(\Theta_{x^{-1}} \otimes f_{i}^{x} \otimes \Theta_{x}\right)\right)(u_{x^{-1}} \otimes \omega_{x} \otimes \underbrace{\bar{u}_{x^{-1}} \otimes \bar{u}_{x}}_{\left(f_{x^{-1, x}}^{\ominus}\right)^{-1}\left(1_{x^{-1}}\right)} b_{l j}) \\
& =\left(f_{x^{-1}, x}^{\Theta} \circ\left(\Theta_{x^{-1}} \otimes f_{i}^{x} \otimes \Theta_{x}\right)\right)\left(u_{x^{-1}} \otimes \omega_{x} \otimes b_{l j} \bar{u}_{x^{-1}} \otimes \bar{u}_{x}\right) \\
& =\left(u_{x^{-1}} f_{i}^{x}\left(\omega_{x} b_{l j} \otimes \bar{u}_{x^{-1}}\right) \stackrel{\ominus}{\circ} \bar{u}_{x}\right) .
\end{aligned}
$$

Voltando, temos:

$$
\begin{aligned}
& \left(f_{x, y}^{\Omega \Gamma} \circ\left(F_{x} \otimes F_{y}\right)\right)\left(v_{x} \otimes u_{x^{-1}} \otimes \omega_{x} \otimes v_{y} \otimes u_{y^{-1}} \otimes \omega_{y}\right) \\
& =\sum\left(\widetilde{\omega}_{x}^{i, p} \stackrel{\Omega}{\circ} \widehat{\omega}_{y}^{j, q}\right) \otimes\left(\widehat{u}_{y^{-1}}^{j, q} \stackrel{\ominus}{\circ} \widetilde{u}_{x^{-1}}^{i, p}\right) \otimes\left(v_{x}\left(u_{x^{-1}} f_{i}^{x}\left(\omega_{x} b_{l, j} \otimes \bar{u}_{x^{-1}}\right) \stackrel{\ominus}{\circ} \bar{u}_{x}\right) \stackrel{\Gamma}{\circ} v_{y}^{l, t}\left(u_{y^{-1}}^{l, t} \stackrel{\ominus}{\circ} \bar{u}_{y}\right)\right) \text {. }
\end{aligned}
$$

Comparando com (3.37) temos que $1_{x} \lambda_{x y} \circ f_{y, x}^{\Gamma \Omega}=f_{x, y}^{\Omega \Gamma} \circ\left(\lambda_{x} \otimes \lambda_{y}\right)$. Logo o diagrama é comutativo e temos

$$
[\Delta(\Omega)][\Delta(\Gamma)]=[\Delta(\Gamma)][\Delta(\Omega)] \text { em } \mathcal{C}(\Theta / R) .
$$

Portanto a multiplicação em $\mathcal{C}(\Theta / R)$ é comutativa.

Considere

$$
\mathcal{C}_{0}(\Theta / R)=\left\{[\Delta(\Gamma)] ; \Gamma_{x} \simeq \Theta_{x}, \text { para todo } x \in G\right\}
$$

Dado $[\Delta(\Gamma)] \in \mathcal{C}_{0}(\Theta / R)$, então $\Gamma_{x} \simeq \Theta_{x}$, para todo $x \in G$. Em particular, $\Gamma_{x} \mid \Theta_{x}$ e

$$
\Gamma_{x} \otimes_{R} \Gamma_{x^{-1}} \simeq \Theta_{x} \otimes_{R} \Theta_{x^{-1}} \simeq R 1_{x} \text {, para todo } x \in G .
$$

Então, $[\Delta(\Gamma)] \in \mathcal{C}(\Theta / R)$. Sejam $[\Delta(\Gamma)],[\Delta(\Omega)] \in \mathcal{C}_{0}(\Theta / R)$, temos

$$
\Gamma_{x} \otimes_{R} \Theta_{x^{-1}} \otimes_{R} \Omega_{x} \simeq \Theta_{x} \otimes_{R} \Theta_{x^{-1}} \otimes_{R} \Theta_{x} \simeq \Theta_{x} \text {, para todo } x \in G .
$$

Assim, $[\Delta(\Gamma)][\Delta(\Omega)]=\left[\bigoplus_{x \in G} \Gamma_{x} \otimes_{R} \Theta_{x^{-1}} \otimes_{R} \Omega_{x}\right] \in \mathcal{C}_{0}(\Theta / R)$. Analogamente, se $[\Delta(\Gamma)] \in \mathcal{C}_{0}(\Theta / R)$, então

$$
\Theta_{x} \otimes_{R} \Gamma_{x^{-1}} \otimes_{R} \Theta_{x} \simeq \Theta_{x} \otimes_{R} \Theta_{x^{-1}} \otimes_{R} \Theta_{x} \simeq \Theta_{x} \text {, para todo } x \in G .
$$

Logo, $[\Delta(\Gamma)]^{-1}=\left[\bigoplus_{x \in G} \Theta_{x} \otimes_{R} \Gamma_{x^{-1}} \otimes_{R} \Theta_{x}\right] \in \mathcal{C}_{0}(\Theta / R)$. Portanto, $\mathcal{C}_{0}(\Theta / R)$ é um subgrupo de $\mathcal{C}(\Theta / R)$. 
Lema 3.4.2. Sejam $[\Delta(\Gamma)] \in \mathcal{C}_{0}(\Theta / R)$ e $a_{x}: \Gamma_{x} \longrightarrow \Theta_{x}$ um isomorfismo de R-bimódulos, para todo $x \in$ G. Considere $\tau_{x, y}: 1_{x} \Theta_{x y} \longrightarrow 1_{x} \Theta_{x y}$ o isomorfismo de R-bimódulos definido pelo diagrama comutativo

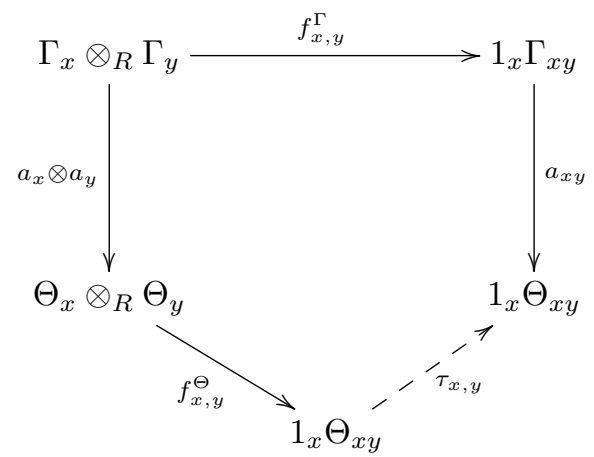

ou seja,

$$
\tau_{x, y} \circ f_{x, y}^{\Theta} \circ\left(a_{x} \otimes a_{y}\right)=a_{x y} \circ f_{x, y}^{\Gamma}, \quad \text { para todos } x, y \in G .
$$

Então, $\widetilde{\tau}_{-,-}$é um elemento normalizado em $Z_{\Theta}^{2}(G, \alpha, \mathcal{Z})$, onde $\widetilde{\tau}_{x, y}$ é como no Lema 3.2.\%.

Demonstração. Pelo Lema 3.2.7 temos que $\widetilde{\tau}_{x, y} \in \mathcal{U}\left(\mathcal{Z} 1_{x} 1_{x y}\right)$ e

$$
\widetilde{\tau}_{x, y}\left(a_{x}\left(v_{x}\right) \stackrel{\ominus}{\circ} a_{y}\left(v_{y}\right)\right)=a_{x y}\left(v_{x} \stackrel{\Gamma}{\circ} v_{y}\right),
$$

para todo $v_{x} \in \Gamma_{x}$ e $v_{y} \in \Gamma_{y}$,

$\operatorname{Dados} x, y, z \in G$ e $v_{x} \in \Gamma_{x}, v_{y} \in \Gamma_{y}$ e $v_{z} \in \Gamma_{z}$, temos

$$
\begin{aligned}
& a_{x y z}\left(\left(v_{x} \stackrel{\Gamma}{\circ} v_{y}\right) \stackrel{\Gamma}{\circ} v_{z}\right) \stackrel{(3.38)}{=} \widetilde{\tau}_{x y, z}\left(a_{x y}\left(v_{x} \stackrel{\Gamma}{\circ} v_{y}\right) \stackrel{\ominus}{\circ} a_{z}\left(v_{z}\right)\right) \\
& \stackrel{(3.38)}{=} \widetilde{\tau}_{x y, z} \widetilde{\tau}_{x, y}\left(\left(a_{x}\left(v_{x}\right) \stackrel{\ominus}{\circ} a_{y}\left(v_{y}\right)\right) \stackrel{\ominus}{\circ} a_{z}\left(v_{z}\right)\right) \\
& =\widetilde{\tau}_{x y, z} \widetilde{\tau}_{x, y}\left(a_{x}\left(v_{x}\right) \stackrel{\ominus}{\circ}\left(a_{y}\left(v_{y}\right) \stackrel{\ominus}{\circ} a_{z}\left(v_{z}\right)\right)\right) \\
& \stackrel{(3.38)}{=} \widetilde{\tau}_{x y, z} \widetilde{\tau}_{x, y}\left(a_{x}\left(v_{x}\right) \stackrel{\ominus}{\circ} \widetilde{\tau}_{y, z}^{-1} a_{y z}\left(v_{y} \circ v_{z}\right)\right) \\
& =\widetilde{\tau}_{x y, z} \widetilde{\tau}_{x, y}\left(a_{x}\left(v_{x}\right) \widetilde{\tau}_{y, z}^{-1} \stackrel{\ominus}{\circ} a_{y z}\left(v_{y} \stackrel{\Gamma}{\circ} v_{z}\right)\right) \\
& \stackrel{(3.14)}{=} \widetilde{\tau}_{x y, z} \widetilde{\tau}_{x, y} \alpha_{x}\left(\widetilde{\tau}_{y, z}^{-1} 1_{x^{-1}}\right)\left(a_{x}\left(v_{x}\right) \stackrel{\ominus}{\circ} a_{y z}\left(v_{y} \stackrel{\Gamma}{\circ} v_{z}\right)\right) \\
& \stackrel{(3.38)}{=} \widetilde{\tau}_{x y, z} \widetilde{\tau}_{x, y} \alpha_{x}\left(\widetilde{\tau}_{y, z}^{-1} 1_{x^{-1}}\right) \widetilde{\tau}_{x, y z}^{-1} a_{x y z}\left(v_{x} \stackrel{\Gamma}{\circ}\left(v_{y} \circ v_{z}\right)\right) \\
& =\widetilde{\tau}_{x y, z} \widetilde{\tau}_{x, y} \alpha_{x}\left(\widetilde{\tau}_{y, z}^{-1} 1_{x^{-1}}\right) \widetilde{\tau}_{x, y z}^{-1} a_{x y z}\left(\left(v_{x} \circ v_{y}\right) \stackrel{\Gamma}{\circ} v_{z}\right) \\
& =a_{x y z}\left(\widetilde{\tau}_{x y, z} \widetilde{\tau}_{x, y} \alpha_{x}\left(\widetilde{\tau}_{y, z}^{-1} 1_{x^{-1}}\right) \widetilde{\tau}_{x, y z}^{-1}\left(v_{x} \circ v_{y}\right) \circ v_{z}\right) \text {. }
\end{aligned}
$$

Como $a_{x y z}$ é isomorfismo de $R$-bimódulos, temos

$$
\left(\left(v_{x} \circ v_{y}\right) \stackrel{\Gamma}{\circ} v_{z}\right)=\widetilde{\tau}_{x y, z} \widetilde{\tau}_{x, y} \alpha_{x}\left(\widetilde{\tau}_{y, z}^{-1} 1_{x^{-1}}\right) \widetilde{\tau}_{x, y z}^{-1}\left(\left(v_{x} \circ v_{y}\right) \circ v_{z}\right),
$$

para todo $v_{x} \in \Gamma_{x}, v_{y} \in \Gamma_{y}$ e $v_{z} \in \Gamma_{z}$. Segue análogo a demonstração da Proposição 3.3.5 que

$$
\alpha_{x}\left(\widetilde{\tau}_{y, z}^{-1} 1_{x^{-1}}\right) \widetilde{\tau}_{x y, z} \widetilde{\tau}_{x, y z}^{-1} \widetilde{\tau}_{x, y}=1_{x} 1_{x y} 1_{x y z}, \text { para todo } x, y, z \in G .
$$

Isso implica que $\widetilde{\tau}_{-,-}^{-1} \in Z_{\Theta}^{2}(G, \alpha, \mathcal{Z})$ e consequentemente, $\widetilde{\tau}_{-,-} \in Z_{\Theta}^{2}(G, \alpha, \mathcal{Z})$. Também análogo a demonstração da Proposição 3.3.5, temos que $\widetilde{\tau}_{-,-}$é normalizado. 
Teorema 3.4.3. A aplicação

$$
\begin{aligned}
& \zeta: \mathcal{C}_{0}(\Theta / R) \quad \longrightarrow \quad H_{\Theta}^{2}(G, \alpha, \mathcal{Z}) \\
& {[\Delta(\Gamma)] \quad \longrightarrow \quad\left[\widetilde{\tau}_{-,-}\right]}
\end{aligned}
$$

onde $\widetilde{\tau}_{-,-} \in Z^{2}(G, \alpha, \mathcal{Z})$ é definido no Lema 3.4.2, é isomorfismo de grupos.

Demonstração. Sejam $[\Delta(\Gamma)],[\Delta(\Omega)]$ em $C_{0}(\Theta / R)$ com isomorfismos de $R$-bimódulos $a_{x}: \Gamma_{x} \longrightarrow \Theta_{x} \mathrm{e}$ $b_{x}: \Omega_{x} \longrightarrow \Theta_{x}$, para todo $x \in G$. Denote,

$$
\begin{aligned}
& \tau_{x, y} \circ f_{x, y}^{\Theta} \circ\left(a_{x} \otimes a_{y}\right)=a_{x y} \circ f_{x, y}^{\Gamma}, \quad \forall x, y \in G, \\
& \gamma_{x, y} \circ f_{x, y}^{\Theta} \circ\left(b_{x} \otimes b_{y}\right)=b_{x y} \circ f_{x, y}^{\Omega}, \quad \forall x, y \in G .
\end{aligned}
$$

Vejamos primeiro que $\zeta$ está bem definida. Suponha $[\Delta(\Gamma)]=[\Delta(\Omega)]$ em $\mathcal{C}_{0}(\Theta / R)$. Então existem isomorfismos de $R$-bimódulos $\xi_{x}: \Gamma_{x} \longrightarrow \Omega_{x}$, para todo $x \in G$, tal que o digrama abaixo é comutativo

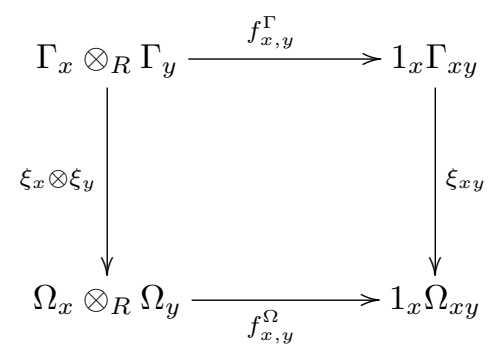

Para cada $x \in G$ considere o isomorfismo de $R$-bimódulos definido por

$$
\beta_{x}: \Theta_{x} \stackrel{a_{x}^{-1}}{\longrightarrow} \Gamma_{x} \stackrel{\xi_{x}}{\longrightarrow} \Omega_{x} \stackrel{b_{x}}{\longrightarrow} \Theta_{x}
$$

ou seja,

$$
\beta_{x}=b_{x} \circ \xi_{x} \circ a_{x}^{-1}, \quad \forall x \in G .
$$

Afirmação 3.4.4. $\beta_{x y} \circ \tau_{x, y} \circ f_{x, y}^{\Theta}=\gamma_{x, y} \circ f_{x, y}^{\Theta} \circ\left(\beta_{x} \otimes \beta_{y}\right)$, para todos $x, y \in G$.

De fato, por (3.40), (3.41) e pela comutatividade do diagrama (3.42), temos

$$
\begin{aligned}
& \gamma_{x, y} \circ f_{x, y}^{\Theta} \circ\left(\beta_{x} \otimes \beta_{y}\right)=\gamma_{x, y} \circ f_{x, y}^{\Theta} \circ\left(\left(b_{x} \circ \xi_{x} \circ a_{x}^{-1}\right) \otimes\left(b_{y} \circ \xi_{y} \circ a_{y}^{-1}\right)\right) \\
& =\gamma_{x, y} \circ f_{x, y}^{\Theta} \circ\left(b_{x} \otimes b_{y}\right) \circ\left(\xi_{x} \otimes \xi_{y}\right) \circ\left(a_{x}^{-1} \otimes a_{y}^{-1}\right) \\
& \stackrel{(3.41)}{=} \quad b_{x y} \circ f_{x, y}^{\Omega} \circ\left(\xi_{x} \otimes \xi_{y}\right) \circ\left(a_{x}^{-1} \otimes a_{y}^{-1}\right) \\
& =b_{x y} \circ \xi_{x y} \circ f_{x, y}^{\Gamma} \circ\left(a_{x}^{-1} \otimes a_{y}^{-1}\right) \\
& \stackrel{(3.40)}{=} b_{x y} \circ \xi_{x y} \circ a_{x y}^{-1} \circ \tau_{x, y} \circ f_{x, y}^{\Theta} \\
& =\beta_{x y} \circ \tau_{x, y} \circ f_{x, y}^{\Theta} \text {. }
\end{aligned}
$$

Então, para todos $u_{x} \in \Theta_{x}, u_{y} \in \Theta_{y}$, temos

$$
\beta_{x y}\left(\tau_{x, y}\left(u_{x} \stackrel{\ominus}{\circ} u_{y}\right)\right)=\gamma_{x, y}\left(\beta_{x}\left(u_{x}\right) \stackrel{\ominus}{\circ} \beta_{y}\left(u_{y}\right)\right) .
$$

Por um lado temos

$$
\beta_{x y}\left(\tau_{x, y}\left(u_{x} \stackrel{\ominus}{\circ} u\right)\right)=\beta_{x y}\left(\widetilde{\tau}_{x, y}\left(u_{x} \stackrel{\ominus}{\circ} u_{y}\right)\right)=\widetilde{\beta}_{x y} \widetilde{\tau}_{x, y}\left(u_{x} \stackrel{\ominus}{\circ} u_{y}\right) .
$$


Por outro lado,

$$
\begin{aligned}
\gamma_{x, y}\left(\beta_{x}\left(u_{x}\right) \stackrel{\ominus}{\circ} \beta_{y}\left(u_{y}\right)\right) & =\widetilde{\gamma}_{x, y}\left(\beta_{x}\left(u_{x}\right) \stackrel{\ominus}{\circ} \beta_{y}\left(u_{y}\right)\right)=\widetilde{\gamma}_{x, y}\left(\widetilde{\beta}_{x} u_{x} \stackrel{\ominus}{\circ} \widetilde{\beta}_{y} u_{y}\right) \\
& =\widetilde{\gamma}_{x, y}\left(\widetilde{\beta}_{x} u_{x} \widetilde{\beta}_{y} \stackrel{\ominus}{\circ} u_{y}\right) \stackrel{(3.14)}{=} \widetilde{\gamma}_{x, y}\left(\widetilde{\beta}_{x} \alpha_{x}\left(\widetilde{\beta}_{y} 1_{x^{-1}}\right) u_{x} \stackrel{\ominus}{\circ} u_{y}\right) \\
& =\widetilde{\gamma}_{x, y} \widetilde{\beta}_{x} \alpha_{x}\left(\widetilde{\beta}_{y} 1_{x^{-1}}\right)\left(u_{x} \stackrel{\ominus}{\circ} u_{y}\right) .
\end{aligned}
$$

Então,

$$
\widetilde{\beta}_{x y} \widetilde{\tau}_{x, y}\left(u_{x} \stackrel{\ominus}{\circ} u_{y}\right)=\widetilde{\gamma}_{x, y} \widetilde{\beta}_{x} \alpha_{x}\left(\widetilde{\beta}_{y} 1_{x-1}\right)\left(u_{x} \stackrel{\ominus}{\circ} u_{y}\right),
$$

para todos $u_{x} \in \Theta_{x}$ e $u_{y} \in \Theta_{y}$. Aplicando o mesmo argumento da Proposição 3.3 .5 temos

$$
\widetilde{\beta}_{x y} \widetilde{\tau}_{x, y}=\widetilde{\gamma}_{x, y} \widetilde{\beta}_{x} \alpha_{x}\left(\widetilde{\beta}_{y} 1_{x^{-1}}\right) \text {, para todos } x, y \in G \text {. }
$$

Defina

$$
\begin{aligned}
h: G & \longrightarrow \mathcal{Z} \\
x & \longmapsto \widetilde{\beta}_{x},
\end{aligned}
$$

então $h_{x} \in \mathcal{U}\left(\mathcal{Z} 1_{x}\right)$, para todo $x \in G$ e temos

$$
\widetilde{\tau}_{x, y}=\widetilde{\gamma}_{x, y} \alpha_{x}\left(h_{y} 1_{x^{-1}}\right) h_{x y}^{-1} h_{x}=\widetilde{\gamma}_{x, y}\left(\delta^{1} h\right)(x, y), \quad \forall x, y \in G .
$$

Isso implica que $\left[\widetilde{\tau}_{-,-}\right]=\left[\widetilde{\gamma}_{-,-}\right]$em $H_{\Theta}^{2}(G, \alpha, \mathcal{Z})$. Portanto, $\zeta$ está bem definida.

Para mostrar que $\zeta$ é injetora, suponha que $\zeta([\Delta(\Gamma)])=\zeta([\Delta(\Omega)])$ em $H_{\Theta}^{2}(G, \alpha, \mathcal{Z})$. Então, existe $h: G \longrightarrow \mathcal{Z}$, com $h_{x} \in \mathcal{U}\left(\mathcal{Z} 1_{x}\right)$, para todo $x \in G$, e

$$
\widetilde{\tau}_{x, y}=\widetilde{\gamma}_{x, y} \alpha_{x}\left(h_{y} 1_{x^{-1}}\right) h_{x y}^{-1} h_{x}, \quad \forall x, y \in G .
$$

Seja

$$
\begin{aligned}
\beta_{x}: \Theta_{x} & \longrightarrow \Theta_{x} \\
u_{x} & \longmapsto h_{x} u_{x},
\end{aligned}
$$

então $\beta_{x}$ é isomorfismo de $R$-bimódulos e $\widetilde{\beta}_{x}=h_{x}$, para todo $x \in G$ (ver Lema 3.2.7). Assim,

$$
\widetilde{\tau}_{x, y} \widetilde{\beta}_{x y}=\widetilde{\gamma}_{x, y} \alpha_{x}\left(\widetilde{\beta}_{y} 1_{x^{-1}}\right) \widetilde{\beta}_{y}, \quad \forall x, y \in G .
$$

Defina o isomorfismo $\xi_{x}$ por

$$
\xi_{x}: \Gamma_{x} \stackrel{a_{x}}{\longrightarrow} \Theta_{x} \stackrel{\beta_{x}}{\longrightarrow} \Theta_{x} \stackrel{b_{x}^{-1}}{\longrightarrow} \Omega_{x}
$$

ou seja,

$$
\xi_{x}=b_{x}^{-1} \circ \beta_{x} \circ a_{x}, \text { para todo } x \in G .
$$

Vamos verificar que o diagrama abaixo é comutativo

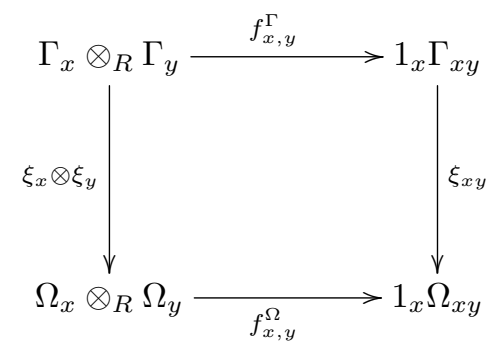

Para isso, vamos precisar da seguinte observação:

Afirmação 3.4.5. $\beta_{x y} \circ \tau_{x, y} \circ f_{x, y}^{\Theta}=\gamma_{x, y} \circ f_{x, y}^{\Theta} \circ\left(\beta_{x} \otimes \beta_{y}\right)$, para todo $x, y \in G$. 
De fato, para $u_{x} \in \Theta_{x}$ e $u_{y} \in \Theta_{y}$, temos

$$
\beta_{x y}\left(\tau_{x, y}\left(u_{x} \stackrel{\ominus}{\circ} u_{y}\right)\right)=\widetilde{\beta}_{x, y} \widetilde{\tau}_{x, y}\left(u_{x} \stackrel{\ominus}{\circ} u_{y}\right) .
$$

Por outro lado,

$$
\begin{aligned}
& \gamma_{x, y}\left(\beta_{x}\left(u_{x}\right) \stackrel{\ominus}{\circ} \beta_{y}\left(u_{y}\right)\right)=\widetilde{\gamma}_{x, y}\left(\widetilde{\beta}_{x} u_{x} \stackrel{\ominus}{\circ} \widetilde{\beta}_{y} u_{y}\right)=\widetilde{\gamma}_{x, y}\left(\widetilde{\beta}_{x} u_{x} \widetilde{\beta}_{y} \stackrel{\ominus}{\circ} u_{y}\right) \\
& =\widetilde{\gamma}_{x, y}\left(\widetilde{\beta}_{x} \alpha_{x}\left(\widetilde{\beta}_{y} 1_{x^{-1}}\right) u_{x} \stackrel{\ominus}{\circ} u_{y}\right) \\
& =\widetilde{\gamma}_{x, y} \widetilde{\beta}_{x} \alpha_{x}\left(\widetilde{\beta}_{y} 1_{x^{-1}}\right)\left(u_{x} \stackrel{\ominus}{\circ} u_{y}\right) \\
& \stackrel{(3.44)}{=} \widetilde{\beta}_{x, y} \widetilde{\tau}_{x, y}\left(u_{x} \stackrel{\ominus}{\circ} u_{y}\right) \\
& \stackrel{(3.46)}{=} \beta_{x y}\left(\tau_{x, y}\left(u_{x} \stackrel{\ominus}{\circ} u_{y}\right)\right) \text {. }
\end{aligned}
$$

Por (3.40), (3.41) e pela Afirmação 3.4.5, temos:

$$
\begin{aligned}
\xi_{x y} \circ f_{x, y}^{\Gamma} & =b_{x y}^{-1} \circ \beta_{x y} \circ a_{x y} \circ f_{x, y}^{\Gamma} \\
& \stackrel{(3.40)}{=} \quad b_{x y}^{-1} \circ \beta_{x y} \circ \tau_{x, y} \circ f_{x, y}^{\Theta} \circ\left(a_{x} \otimes a_{y}\right) \\
& =\quad b_{x y}^{-1} \circ \gamma_{x y} \circ f_{x, y}^{\Theta} \circ\left(\beta_{x} \otimes \beta_{y}\right) \circ\left(a_{x} \otimes a_{y}\right) \\
& \stackrel{(3.41)}{=} \\
& b_{x y}^{-1} \circ b_{x y} \circ f_{x, y}^{\Omega} \circ\left(b_{x}^{-1} \otimes b_{y}^{-1}\right) \circ\left(\beta_{x} \otimes \beta_{y}\right) \circ\left(a_{x} \otimes a_{y}\right) \\
& =f_{x, y}^{\Omega} \otimes\left(\xi_{x} \otimes \xi_{y}\right) .
\end{aligned}
$$

Logo o diagrama (3.45) é comutativo. Assim, temos um isomorfismo de produtos cruzados generalizados parciais. Portanto, $[\Delta(\Gamma)]=[\Delta(\Omega)]$ em $\mathcal{C}_{0}(\Theta / R)$, mostrando que $\zeta$ é injetora.

Seja $\sigma: G \times G \longrightarrow \mathcal{Z}$ um 2-cociclo normalizado em $H_{\Theta}^{2}(G, \alpha, \mathcal{Z})$, então $\sigma_{x, y} \in \mathcal{U}\left(\mathcal{Z} 1_{x} 1_{x y}\right)$ e

$$
\alpha_{x}\left(\sigma_{y, z} 1_{x^{-1}}\right) \sigma_{x, y z}=\sigma_{x y, z} \sigma_{x, y}, \forall x, y, z \in G .
$$

Considere o isomorfismo de $R$-bimódulos

$$
\begin{aligned}
\rho_{x}: \Theta_{x} & \longrightarrow \Theta_{x} \\
u_{x} & \longmapsto \sigma_{x, x} u_{x}
\end{aligned}
$$

e defina $\Sigma_{x}=\Theta_{x}$, como $R$-bimódulos. Então, $\rho_{x}: \Sigma_{x} \longrightarrow \Theta_{x}$ é isomorfismo de $R$-bimódulos. Seja

$$
\begin{aligned}
f_{x, y}^{\Sigma}: \Sigma_{x} \otimes_{R} \Sigma_{y} & \longrightarrow 1_{x} \Sigma_{x y} \\
u_{x} \otimes u_{y} & \longmapsto \sigma_{x, y} f_{x, y}^{\Theta}\left(u_{x} \otimes u_{y}\right) .
\end{aligned}
$$

Como $\sigma$ é um 2-cociclo, então $\left\{f_{x, y}^{\Sigma}\right\}$ é um conjunto de fatores para $\Sigma$. De fato, sejam $u_{x} \in \Sigma_{x}, u_{y} \in \Sigma_{y}$ $\mathrm{e} u_{z} \in \Sigma_{z}$, então

$$
\begin{array}{rll}
f_{x, y z}^{\Sigma}\left(u_{x} \otimes f_{y, z}^{\Sigma}\left(u_{y} \otimes u_{z}\right)\right) & = & \sigma_{x, y z} f_{x, y z}^{\Theta}\left(u_{x} \otimes \sigma_{y, z} f_{x, y}^{\Theta}\left(u_{y} \otimes u_{z}\right)\right) \\
& = & \sigma_{x, y z} f_{x, y z}^{\Theta}\left(u_{x} \sigma_{y, z} \otimes f_{x, y}^{\Theta}\left(u_{y} \otimes u_{z}\right)\right) \\
& \stackrel{(3.14)}{=} & \sigma_{x, y z} \alpha_{x}\left(\sigma_{y, z} 1_{x-1}\right) f_{x, y z}^{\Theta}\left(u_{x} \otimes f_{x, y}^{\Theta}\left(u_{y} \otimes u_{z}\right)\right) \\
& \stackrel{(3.47)}{=} & \sigma_{x y, z} \sigma_{x, y} f_{x y, z}^{\Theta}\left(f_{x, y}^{\Theta}\left(u_{x} \otimes u_{y}\right) \otimes u_{z}\right) \\
& = & \sigma_{x y, z} f_{x y, z}^{\Theta}\left(\sigma_{x, y} f_{x, y}^{\Theta}\left(u_{x} \otimes u_{y}\right) \otimes u_{z}\right) \\
& = & f_{x y, z}^{\Sigma}\left(f_{x, y}^{\Sigma}\left(u_{x} \otimes u_{y}\right) \otimes u_{z}\right) .
\end{array}
$$


Assim, $[\Delta(\Sigma)] \in \mathcal{C}_{0}(\Theta / R)$. Defina

$$
\begin{aligned}
\lambda: \quad & \longrightarrow \mathcal{Z} \\
x & \longmapsto \lambda_{x}=\sigma_{x, x}^{-1}
\end{aligned}
$$

Então $\lambda_{x} \in \mathcal{U}\left(\mathcal{Z} 1_{x}\right)$, para todo $x \in G$. Agora, observe que

$$
\begin{aligned}
\rho_{x y}\left(f_{x, y}^{\Sigma}\left(u_{x} \otimes u_{y}\right)\right) & =\rho_{x y}\left(\sigma_{x, y} f_{x, y}^{\Theta}\left(u_{x} \otimes u_{y}\right)\right) \\
& =\sigma_{x, y} \rho_{x y}\left(f_{x, y}^{\Theta}\left(u_{x} \otimes u_{y}\right)\right) \\
& =\sigma_{x, y} \sigma_{x y, x y} f_{x, y}^{\Theta}\left(u_{x} \otimes u_{y}\right) \\
& =\sigma_{x, y} \sigma_{x y, x y} f_{x, y}^{\Theta}\left(\sigma_{x, x}^{-1} \rho_{x}\left(u_{x}\right) \otimes \sigma_{y, y}^{-1} \rho_{y}\left(u_{y}\right)\right) \\
& =\sigma_{x, y} \sigma_{x y, x y} f_{x, y}^{\Theta}\left(\sigma_{x, x}^{-1} \rho_{x}\left(u_{x}\right) \sigma_{y, y}^{-1} \otimes \rho_{y}\left(u_{y}\right)\right) \\
& =\sigma_{x, y} \sigma_{x y, x y} f_{x, y}^{\Theta}\left(\sigma_{x, x}^{-1} \alpha_{x}\left(\sigma_{y, y}^{-1} 1_{x-1}\right) \rho_{x}\left(u_{x}\right) \otimes \rho_{y}\left(u_{y}\right)\right) \\
& =\sigma_{x, y} \sigma_{x y, x y} \sigma_{x, x}^{-1} \alpha_{x}\left(\sigma_{y, y}^{-1} 1_{x^{-1}}\right) f_{x, y}^{\Theta}\left(\rho_{x}\left(u_{x}\right) \otimes \rho_{y}\left(u_{y}\right)\right) \\
& =\sigma_{x, y}\left(\delta^{1} \lambda\right)(x, y) f_{x, y}^{\Theta}\left(\rho_{x}\left(u_{x}\right) \otimes \rho_{y}\left(u_{y}\right)\right) .
\end{aligned}
$$

Logo,

$$
\zeta([\Delta(\Sigma)])=\left[\sigma\left(\delta^{1} \lambda\right)\right]=[\sigma] \text { em } H_{\Theta}^{2}(G, \alpha, \mathcal{Z})
$$

Portanto, $\zeta$ é sobrejetora.

Por fim, vejamos que $\zeta$ é morfismo de grupos. Por definição temos que $[\Delta(\Gamma)][\Delta(\Omega)]=\left[\bigoplus_{x \in G} \Gamma_{x} \otimes \Theta_{x^{-1}} \otimes \Omega_{x}\right]$ em $\mathcal{C}_{0}(\Theta / R)$. Considere o isomorfismo de $R$-bimódulos

$$
\begin{aligned}
d_{x}: \Gamma_{x} \otimes_{R} \Theta_{x^{-1}} \otimes_{R} \Omega_{x} & \longrightarrow \Theta_{x} \\
v_{x} \otimes u_{x^{-1}} \otimes \omega_{x} & \longmapsto a_{x}\left(v_{x}\right)\left(u_{x^{-1}} \stackrel{\ominus}{\circ} b_{x}\left(\omega_{x}\right)\right) .
\end{aligned}
$$

Observe que $d_{x y} \circ f_{x, y}^{\Gamma \Omega}=d_{x y} \circ\left(f_{x, y}^{\Gamma} \otimes f_{y^{-1}, x^{-1}}^{\Theta} \otimes f_{x y}^{\Omega}\right) \circ\left(\Gamma_{x} \otimes T \otimes \Omega_{y}\right)$, onde $T$ é o isomorfismo

$$
T: \Theta_{x^{-1}} \otimes_{R} \Omega_{x} \otimes_{R} \Gamma_{y} \otimes_{R} \Theta_{y^{-1}} \longrightarrow \Gamma_{y} \otimes_{R} \Theta_{y^{-1}} \otimes_{R} \Theta_{x^{-1}} \otimes_{R} \Omega_{x}
$$

Defina

$$
\begin{gathered}
f_{x}: \Theta_{x^{-1}} \otimes_{R} \Omega_{x} \stackrel{\Theta_{x-1} \otimes b_{x}}{\longrightarrow} \Theta_{x^{-1}} \otimes_{R} \Theta_{x} \longrightarrow R 1_{x^{-1}} \hookrightarrow R, \\
g_{x}: R \longrightarrow R 1_{x^{-1}} \longrightarrow \Theta_{x^{-1}} \otimes_{R} \Theta_{x} \stackrel{\Theta_{x-1} \otimes b_{x}^{-1}}{\longrightarrow} \Theta_{x^{-1}} \otimes_{R} \Omega_{x} .
\end{gathered}
$$

É fácil ver que $g_{x} f_{x}=I d_{\Theta_{x-1} \otimes \Omega_{x}}$. Então, podemos escrever o isomorfismo $T$ em função de $f_{x}$ e $g_{x}$.

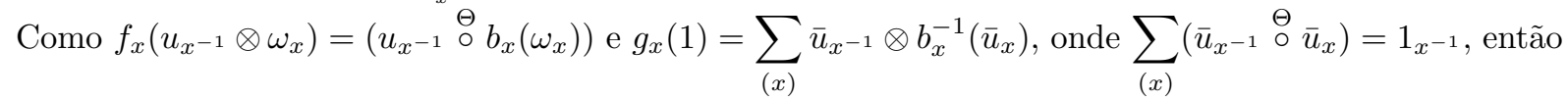

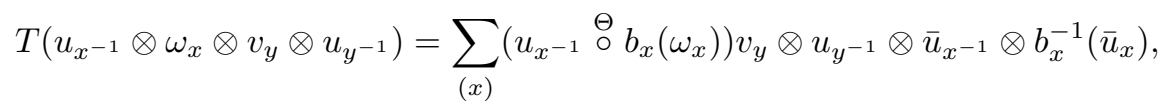


Assim, $d_{x y} \circ f_{x, y}^{\Gamma \Omega}$ :

$$
\begin{aligned}
& v_{x} \otimes u_{x^{-1}} \otimes \omega_{x} \otimes v_{y} \otimes u_{y^{-1}} \otimes \omega_{y} \\
& \stackrel{T}{\mapsto} \sum_{(x)} v_{x} \otimes\left(u_{x^{-1}} \stackrel{\ominus}{\circ} b_{x}\left(\omega_{x}\right)\right) v_{y} \otimes u_{y^{-1}} \otimes \bar{u}_{x^{-1}} \otimes b_{x}^{-1}\left(\bar{u}_{x}\right) \otimes \omega_{y}
\end{aligned}
$$

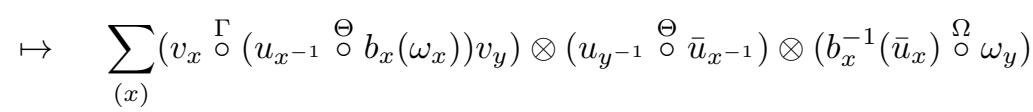

$$
\begin{aligned}
& \left.\stackrel{d_{x y}}{\mapsto} \sum_{(x)} a_{x y}\left(v_{x}\left(u_{x^{-1}} \stackrel{\ominus}{\circ} b_{x}\left(\omega_{x}\right)\right)\right) \stackrel{\Gamma}{\circ} v_{y}\right)\left(\left(u_{y^{-1}} \stackrel{\ominus}{\circ} \bar{u}_{x^{-1}}\right) \stackrel{\ominus}{\circ} b_{x y}\left(b_{x}^{-1}\left(\bar{u}_{x}\right) \stackrel{\Omega}{\circ} \omega_{y}\right)\right)
\end{aligned}
$$

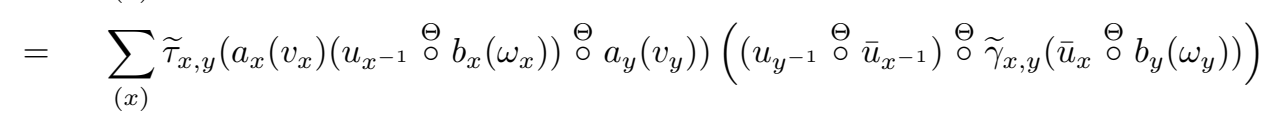

$$
\begin{aligned}
& \stackrel{(3.14)}{=} \sum_{(x)} \widetilde{\tau}_{x, y} \widetilde{\gamma}_{x, y}\left(a_{x}\left(v_{x}\right)\left(u_{x^{-1}} \stackrel{\ominus}{\circ} b_{x}\left(\omega_{x}\right)\right) \stackrel{\ominus}{\circ} a_{y}\left(v_{y}\right)\right)\left(\left(u_{y^{-1}} \stackrel{\ominus}{\circ} \bar{u}_{x^{-1}}\right) \stackrel{\ominus}{\circ}\left(\bar{u}_{x} \stackrel{\ominus}{\circ} b_{y}\left(\omega_{y}\right)\right)\right)
\end{aligned}
$$

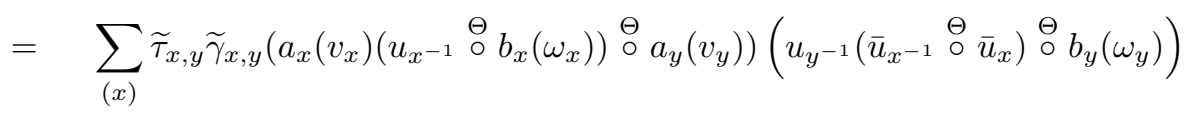

$$
\begin{aligned}
& =\widetilde{\tau}_{x, y} \widetilde{\gamma}_{x, y}\left(a_{x}\left(v_{x}\right)\left(u_{x^{-1}} \stackrel{\ominus}{\circ} b_{x}\left(\omega_{x}\right)\right) \stackrel{\ominus}{\circ} a_{y}\left(v_{y}\right)\right)\left(u_{y^{-1}} 1_{x^{-1}} \stackrel{\ominus}{\circ} b_{y}\left(\omega_{y}\right)\right) \\
& \stackrel{(3.10)}{=} \widetilde{\tau}_{x, y} \widetilde{\gamma}_{x, y}\left(a_{x}\left(v_{x}\right)\left(u_{x^{-1}} \stackrel{\ominus}{\circ} b_{x}\left(\omega_{x}\right)\right) \stackrel{\ominus}{\circ} a_{y}\left(v_{y}\right) 1_{(x y)^{-1}}\left(u_{y^{-1}} \stackrel{\ominus}{\circ} b_{y}\left(\omega_{y}\right)\right)\right) \\
& \stackrel{(3.10)}{=} \widetilde{\tau}_{x, y} \widetilde{\gamma}_{x, y}\left(a_{x}\left(v_{x}\right)\left(u_{x^{-1}} \stackrel{\ominus}{\circ} b_{x}\left(\omega_{x}\right) 1_{x^{-1}}\right) \stackrel{\ominus}{\circ} a_{y}\left(v_{y}\right)\left(u_{y^{-1}} \stackrel{\ominus}{\circ} b_{y}\left(\omega_{y}\right)\right)\right) \\
& =\widetilde{\tau}_{x, y} \widetilde{\gamma}_{x, y}\left(a_{x}\left(v_{x}\right)\left(u_{x^{-1}} \stackrel{\ominus}{\circ} b_{x}\left(\omega_{x}\right)\right) \stackrel{\ominus}{\circ} a_{y}\left(v_{y}\right)\left(u_{y^{-1}} \stackrel{\ominus}{\circ} b_{y}\left(\omega_{y}\right)\right)\right) \\
& =\quad \widetilde{\tau}_{x, y} \widetilde{\gamma}_{x, y}\left(d_{x}\left(v_{x} \otimes u_{x^{-1}} \otimes \omega_{x}\right) \stackrel{\ominus}{\circ} d_{y}\left(v_{y} \otimes u_{y^{-1}} \otimes \omega_{y}\right)\right) .
\end{aligned}
$$

Logo,

$$
\tau_{x, y} \circ \gamma_{x, y} \circ f_{x, y}^{\Theta} \circ\left(d_{x} \otimes d_{y}\right)=d_{x y} \circ f_{x, y}^{\Gamma \Omega}, \quad \forall x, y \in G .
$$

Portanto, $\zeta([\Delta(\Gamma)][\Delta(\Omega)])=\left[\widetilde{\tau}_{-,-} \widetilde{\gamma}_{-,-}\right]=\zeta([\Delta(\Gamma)]) \zeta([\Delta(\Omega)])$. 


\section{Capítulo 4}

\section{A sequência exata de sete termos}

Nesse Capítulo vamos construir a versão parcial da sequência de Miyashita para anéis não comutativos com unidade. A sequência construída nesse capítulo generaliza a sequência dada em [5, Teorema 2.12]. Dados uma extensão de anéis com mesma unidade $R \subseteq S$ e uma representação parcial unital

$$
\begin{aligned}
\Theta: G & \longrightarrow \mathcal{S}_{R}(S) \\
x & \longmapsto \Theta_{x}
\end{aligned}
$$

com $\varepsilon_{x}=\Theta_{x} \Theta_{x^{-1}}=R 1_{x}$, para todo $x \in G$. Considere $\Delta(\Theta)$ o produto cruzado generalizado parcial construído através de $\Theta$ como na Observação 3.3.13. Denotamos $f^{\Theta}=\left\{f_{x, y}^{\Theta}: \Theta_{x} \otimes \Theta_{y} \longrightarrow 1_{x} \Theta_{x y}, x, y \in\right.$ $G\}$ o conjunto de fatores de $\Theta$ e $\iota: R \longrightarrow \Theta_{1}$ o isomorfismo de anéis como na Observação 3.3.4.

Em todo o Capítulo $\otimes$ significa $\otimes_{R}$, a menos de menção contrária.

\subsection{A primeira sequência exata}

Seja $\mathcal{P}_{\mathcal{Z}}(S / R)$ o grupo definido na Seção 2.4. Definimos

$$
\mathcal{P}_{\mathcal{Z}}(S / R)^{(G)}=\left\{([P]=[\phi] \Longrightarrow[X]) \in \mathcal{P}_{\mathcal{Z}}(S / R) ; \Theta_{x} \phi(P)=\phi(P) \Theta_{x} \text { para todo } x \in G\right\} \text {. }
$$

Observação 4.1.1. Seja $([P]=[\phi] \Longrightarrow[X]) \in \mathcal{P}_{\mathcal{Z}}(S / R)$. Então,

$$
\Theta_{x} \phi(P)=\phi(P) \Theta_{x} \text { se, e somente se, } \Theta_{x} \phi(P) \Theta_{x^{-1}}=\phi(P) 1_{x} \text {, para todo } x \in G \text {. }
$$

De fato, como $P$ é ZZ-bimódulo central, então $P 1_{x}=1_{x} P$, para todo $x \in G$. Assim, pela R-bilinearidade de $\phi$ temos $1_{x} \phi(P)=\phi(P) 1_{x}$, para todo $x \in G$. Se $\Theta_{x} \phi(P)=\phi(P) \Theta_{x}$, para todo $x \in G$, então

$$
\Theta_{x} \phi(P) \Theta_{x^{-1}}=\phi(P) \Theta_{x} \Theta_{x^{-1}}=\phi(P) 1_{x}, \text { para todo } x \in G \text {. }
$$

Por outro lado,

$$
\begin{aligned}
\Theta_{x} \phi(P) \Theta_{x^{-1}}=\phi(P) 1_{x} & \Rightarrow \Theta_{x} \phi(P) \Theta_{x^{-1}} \Theta_{x}=\phi(P) 1_{x} \Theta_{x} \\
& \Rightarrow \Theta_{x} \phi(P) 1_{x^{-1}}=\phi(P) \Theta_{x} \\
& \Rightarrow \Theta_{x} 1_{x^{-1}} \phi(P)=\phi(P) \Theta_{x} \\
& \Rightarrow \Theta_{x} \phi(P)=\phi(P) \Theta_{x}
\end{aligned}
$$


Lema 4.1.2. $\mathcal{P}_{\mathcal{Z}}(S / R)^{(G)}$ é um subgrupo de $\mathcal{P}_{\mathcal{Z}}(S / R)$.

Demonstração. É fácil ver que $\mathcal{P}_{\mathcal{Z}}(S / R)^{(G)}$ é fechado para o produto. Vejamos que é fechado para o inverso. Seja $[P]=[\phi] \Longrightarrow[X] \in \mathcal{P}_{\mathcal{Z}}(S / R)^{(G)}$. Pela Proposição 2.4.4, seu inverso em $\mathcal{P}_{\mathcal{Z}}(S / R)$ é dado por $\left[P^{*}\right]=\left[\phi^{*}\right] \Rightarrow\left[X^{*}\right]$. Como $\phi$ é injetora podemos ver $P \subseteq X$ e $P^{*} \subseteq X^{*}$. Observe que $P^{*}=\left\{f \in X^{*} ; f(P) \subseteq R\right\}$. Assim, $P^{*} \cdot 1_{x}=\left\{f \in X^{*} ; f(P) \subseteq R 1_{x}\right\}$. De fato, seja $f \in X^{*}$, tal que $f(P) \subseteq R 1_{x}$, então $f \in P^{*}$ e

$$
\left(f \cdot 1_{x}\right)(p)=f(p) 1_{x}=f(p) .
$$

Logo, $f \in P^{*} \cdot 1_{x}$. Por outro lado, se $f \in P^{*} \cdot 1_{x}$, então $f=f^{\prime} \cdot 1_{x}$, para algum $f^{\prime} \in P^{*}$. Então,

$$
f(p)=\left(f^{\prime} \cdot 1_{x}\right)(p)=f^{\prime}(p) 1_{x} \in R 1_{x}, \text { para todo } p \in P .
$$

Portanto, vale a igualdade. Vejamos agora que $\Theta_{x} \cdot P^{*} \cdot \Theta_{x^{-1}}=P^{*} \cdot 1_{x}$, para todo $x \in G$. Temos,

$$
\begin{aligned}
\left(\Theta_{x} \cdot P^{*} \cdot \Theta_{x^{-1}}\right)(P) & =\left(P^{*} \cdot \Theta_{x^{-1}}\right)\left(P \Theta_{x}\right)=\left(P^{*} \cdot \Theta_{x^{-1}}\right)\left(\Theta_{x} P\right) \\
& =\Theta_{x}\left(P^{*} \cdot \Theta_{x^{-1}}\right)(P)=\Theta_{x}\left[P^{*}(P)\right] \Theta_{x^{-1}} \\
& \subseteq \Theta_{x} R \Theta_{x^{-1}}=R 1_{x} .
\end{aligned}
$$

Logo, $\Theta_{x} \cdot P^{*} \cdot \Theta_{x^{-1}} \subseteq P^{*} \cdot R 1_{x}$, para todo $x \in G$. Agora, observe que

$$
\begin{aligned}
P^{*} \cdot R 1_{x} & =R 1_{x} \cdot P^{*} \cdot R 1_{x}=\left(\Theta_{x} \Theta_{x^{-1}}\right) \cdot P^{*} \cdot\left(\Theta_{x} \Theta_{x^{-1}}\right) \\
& =\Theta_{x} \cdot\left(\Theta_{x^{-1}} \cdot P^{*} \cdot \Theta_{x}\right) \cdot \Theta_{x^{-1}} \\
& \subseteq \Theta_{x} \cdot\left(P^{*} \cdot R 1_{x^{-1}}\right) \cdot \Theta_{x^{-1}} \\
& =\Theta_{x} \cdot P^{*} \cdot\left(R 1_{x^{-1}} \Theta_{x^{-1}}\right) \\
& =\Theta_{x} \cdot P^{*} \cdot \Theta_{x^{-1}} .
\end{aligned}
$$

Logo vale a igualdade. Portanto, $\left[P^{*}\right]=\left[\phi^{*}\right] \Rightarrow\left[X^{*}\right] \in \mathcal{P}_{\mathcal{Z}}(S / R)^{(G)}$.

Proposição 4.1.3. A aplicação

$$
\begin{aligned}
& \varphi_{2}: \quad \mathcal{P}_{\mathcal{Z}}(S / R)^{(G)} \quad \longrightarrow \quad \operatorname{Pic}_{\mathcal{Z}}(R) \cap \mathbf{P i c S}_{\mathcal{Z}}(R)^{\alpha^{*}} \\
& ([P]=[\phi] \Longrightarrow[X]) \longmapsto[P]
\end{aligned}
$$

é um morfismo de grupos bem definido.

Demonstração. Claramente $\varphi_{2}$ é morfismo de grupos. Basta mostrar que se $[P]=[\phi] \Longrightarrow[X] \in$ $\mathcal{P}_{\mathcal{Z}}(S / R)^{(G)}$, então $[P] \in \mathbf{P i c S}_{\mathcal{Z}}(R)^{\alpha^{*}}$. Por (4.1) temos que $\Theta_{x} \phi(P) \Theta_{x^{-1}}=\phi\left(P R 1_{x}\right)$, para todo $x \in G$. Como $\phi$ é $R$-bilinear, então $\phi(P)$ é um $R$-subbimodulo de $X$. Pela Proposição 3.3.11 e por $\phi$ ser injetora, temos que $f_{x}$ definida por

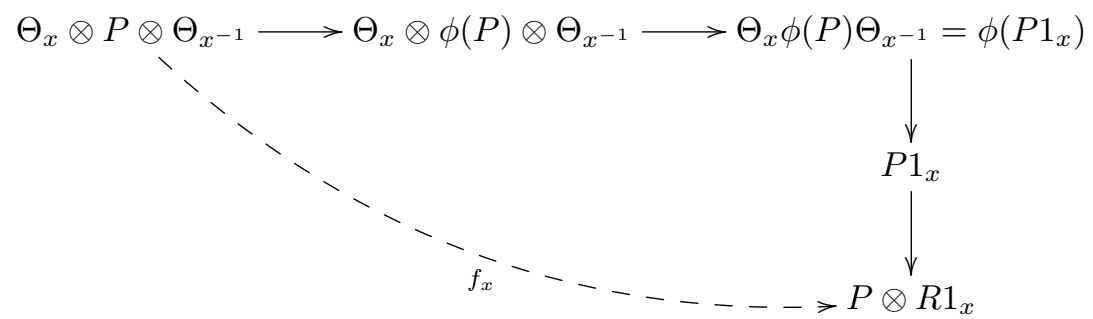

ou seja,

$$
f_{x}\left(u_{x} \otimes p \otimes u_{x^{-1}}\right)=p^{\prime} \otimes 1_{x}
$$


onde $\phi\left(p^{\prime}\right)=u_{x} \phi(p) u_{x^{-1}}$, é um isomorfismo de $R$-bimódulos. Logo, $[P] \in \mathbf{P i c}_{\mathcal{Z}}(R)^{\alpha^{*}}$.

Vamos denotar

$$
\operatorname{Aut}_{R \text {-rings }}(S)^{(G)}=\left\{f \in \mathbf{A u t}_{R \text {-rings }}(S) ; f\left(\Theta_{x}\right)=\Theta_{x}, \forall x \in G\right\} .
$$

Claramente, $\mathbf{A u t}_{R \text {-rings }}(S)^{(G)}$ é um subgrupo de $\operatorname{Aut}_{R \text {-rings }}(S)$.

Lema 4.1.4. O diagrama abaixo é comutativo com linhas exatas

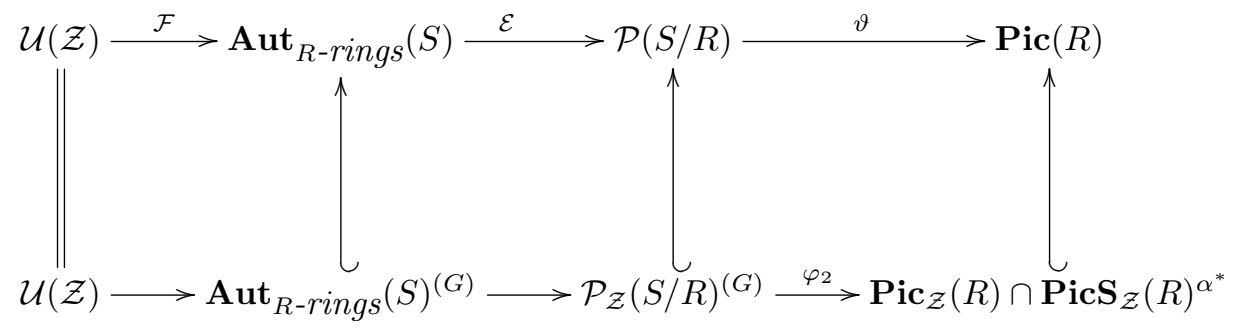

Demonstração. A exatidão da primeira linha é dada no Teorema 2.5.1. A restrição de $\vartheta$ a $\mathcal{P}_{\mathcal{Z}}(S / R)^{(G)}$ é o morfismo $\varphi_{2}$ definido em Proposição 4.1.3. Seja $r \in \mathcal{U}(\mathcal{Z})$, como $\Theta_{x}$ é $R$-bimódulo, temos que $\mathcal{F}(r) \in \mathbf{A u t}_{R \text {-rings }}(S)^{(G)}$. Dado $f \in \mathbf{A u t}_{R \text {-rings }}(S)^{(G)}$, então

$$
i_{f}(R) \cdot \Theta_{x}=R f\left(\Theta_{x}\right)=R \Theta_{x}=\Theta_{x} R=\Theta_{x} \cdot i_{f}(R) \text {, para todo } x \in G .
$$

Como $R$ é $\mathcal{Z}$-bimódulo central, então $\mathcal{E}(f)=\left([R]={ }^{\left[\iota_{f}\right]} \Rightarrow\left[S_{f}\right]\right) \in \mathcal{P}_{\mathcal{Z}}(S / R)^{(G)}$. Logo, os morfismos da segunda linha estão bem definidas. Vejamos que a segunda linha é exata.

A exatidão no primeiro termo e a inclusão $\mathcal{E}\left(\operatorname{Aut}_{R \text {-Rings }}(S)^{(G)}\right) \subseteq \operatorname{ker}\left(\varphi_{2}\right)$ segue diretamente da exatidão da primeira linha. Seja $[P]=[\phi] \Longrightarrow[X] \in \operatorname{ker}\left(\varphi_{2}\right)$. Então, existe $f \in \operatorname{Aut}_{R \text {-rings }}(S)$ tal que $\mathcal{E}(f)=\left([R]=\left[\iota_{f}\right] \Rightarrow\left[S_{f}\right]\right)=([P]=[\phi] \Longrightarrow[X])$ em $\mathcal{P}(S / R)$. Pela demonstração do Teorema 2.5.1 temos que se $\lambda: R \longrightarrow P$ é um isomorfismo de $R$-bimódulos, definindo $\alpha$ e $\beta$ por

$$
\begin{gathered}
\alpha: S \longrightarrow R \otimes_{R} S \longrightarrow P \otimes_{R} S \stackrel{\bar{\phi}_{r}}{\longrightarrow} X \\
\beta: S \longrightarrow S \otimes_{R} R \longrightarrow S \otimes_{R} P \stackrel{\bar{\phi}_{l}}{\longrightarrow} X
\end{gathered}
$$

então $f=\beta^{-1} \circ \alpha$ e o diagrama

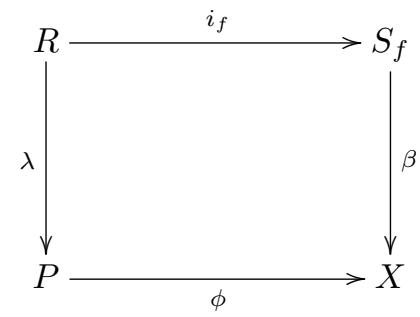

é comutativo. Por $[P]=[\phi] \Longrightarrow[X] \in \mathcal{P}_{\mathcal{Z}}(S / R)^{(G)}$, então $\phi(P) \Theta_{x}=\Theta_{x} \phi(P)$, para todo $x \in G$. Vamos verificar que $f\left(\Theta_{x}\right)=\Theta_{x}$, para todo $x \in G$.

Dado $u_{x} \in \Theta_{x}$, temos que $\phi(\lambda(1)) u_{x} \in \phi(P) \Theta_{x}=\Theta_{x} \phi(P)$, assim existem $u_{x}^{i} \in \Theta_{x}$ e $p_{i} \in P$ com $i=1,2, \ldots, n$, tais que $\phi(\lambda(1)) u_{x}=\sum_{i=1}^{n} u_{x}^{i} \phi\left(p_{i}\right)$. Como $\lambda$ é isomorfismo existem $r_{i} \in R$ tais que $\lambda\left(r_{i}\right)=p_{i}$, 
para todo $i=1,2, \ldots, n$. Logo, temos

$$
\begin{aligned}
\alpha\left(u_{x}\right) & =\phi(\lambda(1)) u_{x}=\sum_{i=1}^{n} u_{x}^{i} \phi\left(p_{i}\right) \\
& =\sum_{i=1}^{n} u_{x}^{i} \phi\left(\lambda\left(r_{i}\right)\right)=\sum_{i=1}^{n} u_{x}^{i} \beta\left(i_{f}\left(r_{i}\right)\right) \\
& =\sum_{i=1}^{n} u_{x}^{i} \beta\left(r_{i}\right)=\sum_{i=1}^{n} \beta\left(u_{x}^{i} r_{i}\right) .
\end{aligned}
$$

Assim,

$$
f\left(u_{x}\right)=\left(\beta^{-1} \circ \alpha\right)\left(u_{x}\right)=\sum_{i=1}^{n} u_{x}^{i} r_{i} \in \Theta_{x} .
$$

Logo, $f\left(\Theta_{x}\right) \subseteq \Theta_{x}$, para todo $x \in G$.

Por outro lado, dado $u_{x} \in \Theta_{x}$ temos $u_{x} \phi(\lambda(1)) \in \Theta_{x} \phi(P)$ e assim existem $v_{x}^{j} \in \Theta_{x}$ e $p_{j} \in P$ com $j=1, \ldots, m$, tais que $u_{x} \phi(\lambda(1))=\sum_{j=1}^{m} \phi\left(p_{j}\right) v_{x}^{j}$. Novamente por $\lambda$ ser isomorfismo existem $r_{j} \in R$ tais que $\lambda\left(r_{j}\right)=p_{j}$ para $j=1, \ldots, m$. Então,

$$
\begin{aligned}
\beta\left(u_{x}\right) & =u_{x} \phi(\lambda(1))=\sum_{j=1}^{m} \phi\left(p_{j}\right) v_{x}^{j}=\sum_{j=1}^{m} \phi\left(\lambda\left(r_{j}\right)\right) v_{x}^{j} \\
& =\sum_{j=1}^{m} \phi(\lambda(1)) r_{j} v_{x}^{j}=\alpha\left(\sum_{j=1}^{m} r_{j} v_{x}^{j}\right) .
\end{aligned}
$$

Assim,

$$
u_{x}=\left(\beta^{-1} \circ \alpha\right)\left(\sum_{j=1}^{m} r_{j} v_{x}^{j}\right)=f\left(\sum_{j=1}^{m} r_{j} v_{x}^{j}\right) \in f\left(\Theta_{x}\right) .
$$

Logo, $\Theta_{x} \subseteq f\left(\Theta_{x}\right)$ e portanto temos a igualdade. Segue então $f \in \mathbf{A u t}_{R \text {-rings }}(S)^{(G)}$ e portanto $[P]=[\phi] \Longrightarrow[X] \in \mathcal{E}\left(\right.$ Aut $\left._{R \text {-rings }}(S)^{(G)}\right)$.

Como $R \subseteq \Delta(\Theta)$ é uma extensão de anéis com mesma unidade, aplicando o Lema 4.1.4 para essa extensão temos:

Teorema 4.1.5. Existe uma sequência exata

$$
1 \longrightarrow H_{\Theta}^{1}(G, \alpha, \mathcal{Z}) \stackrel{\varphi_{1}}{\longrightarrow} \mathcal{P}_{\mathcal{Z}}(\Delta(\Theta) / R)^{(G)} \stackrel{\varphi_{2}}{\longrightarrow} \operatorname{Pic}_{\mathcal{Z}}(R) \cap \operatorname{PicS}_{\mathcal{Z}}(R)^{\alpha^{*}} .
$$

Demonstração. Pelo Lema 4.1.4 a sequência

$$
\mathcal{U}(\mathcal{Z}) \stackrel{\mathcal{F}}{\longrightarrow} \operatorname{Aut}_{R \text {-rings }}(\Delta(\Theta))^{(G)} \longrightarrow \mathcal{P}_{\mathcal{Z}}(\Delta(\Theta) / R)^{(G)} \stackrel{\varphi_{2}}{\longrightarrow} \operatorname{Pic}_{\mathcal{Z}}(R) \cap \operatorname{PicS}_{\mathcal{Z}}(R)^{\alpha^{*}}
$$

é exata. Logo,

$$
1 \longrightarrow \frac{\operatorname{Aut}_{R \text {-rings }}(\Delta(\Theta))^{(G)}}{\operatorname{Im}(\mathcal{F})} \longrightarrow \mathcal{P}_{\mathcal{Z}}(\Delta(\Theta) / R)^{(G)} \stackrel{\varphi_{2}}{\longrightarrow} \operatorname{Pic}_{\mathcal{Z}}(R) \cap \operatorname{PicS}_{\mathcal{Z}}(R)^{\alpha^{*}}
$$


é exata. Basta então mostrar que existe um isomorfismo de grupos $\frac{\operatorname{Aut}_{R \text {-rings }}(\Delta(\Theta))^{(G)}}{\operatorname{Im}(\mathcal{F})} \simeq H_{\Theta}^{1}(G, \alpha, \mathcal{Z})$.

Seja $f \in \mathbf{A u t}_{R \text {-rings }}(\Delta(\Theta))^{(G)}$. Como $f\left(\Theta_{x}\right)=\Theta_{x}$, para todo $x \in G$ e $f$ fixa cada elemento de $R$, então restringindo $f$ a $\Theta_{x}$, temos isomorfismos de $R$-bimódulos $f_{x}: \Theta_{x} \longrightarrow \Theta_{x}$, para cada $x \in G$. Seja $\widetilde{f}_{x} \in \mathcal{U}\left(\mathcal{Z} 1_{x}\right)$ como no Lema 3.2.7. Por $f$ ser automorfismo de anéis temos $f\left(u_{x} \stackrel{\ominus}{\circ} u_{y}\right)=f\left(u_{x}\right) \stackrel{\ominus}{\circ} f\left(u_{y}\right)$, para todo $u_{x} \in \Theta_{x}$ e $u_{y} \in \Theta_{y}$. Então,

$$
f_{x y}\left(u_{x} \stackrel{\ominus}{\circ} u_{y}\right)=f_{x}\left(u_{x}\right) \stackrel{\ominus}{\circ} f_{y}\left(u_{y}\right), u_{x} \in \Theta_{x}, u_{y} \in \Theta_{y} .
$$

Por (3.17) temos

$$
\begin{gathered}
f_{x y}\left(u_{x} \stackrel{\ominus}{\circ} u_{y}\right)=\widetilde{f_{x y}}\left(u_{x} \stackrel{\ominus}{\circ} u_{y}\right), \\
f_{x}\left(u_{x}\right) \stackrel{\ominus}{\circ} f_{y}\left(u_{y}\right) \stackrel{\left(=f_{x} u_{x} \stackrel{\ominus}{\circ} \widetilde{f}_{y} u_{y}=\widetilde{f}_{x} u_{x} \widetilde{f}_{y} \stackrel{\ominus}{\circ} u_{y}\right.}{\stackrel{(3.14)}{=} \widetilde{f}_{x} \alpha_{x}\left(\widetilde{f}_{y} 1_{x^{-1}}\right)\left(u_{x} \stackrel{\ominus}{\circ} u_{y}\right) .}
\end{gathered}
$$

Logo, $\widetilde{f_{x y}}\left(u_{x} \stackrel{\ominus}{\circ} u_{y}\right)=\widetilde{f}_{x} \alpha_{x}\left(\widetilde{f}_{y} 1_{x^{-1}}\right)\left(u_{x} \stackrel{\ominus}{\circ} u_{y}\right)$, para todo $u_{x} \in \Theta_{x}$ e $u_{y} \in \Theta_{y}$. Seguindo o argumento da Proposição 3.3.5, temos

$$
\widetilde{f}_{x} \alpha_{x}\left(\widetilde{f}_{y} 1_{x^{-1}}\right)=\widetilde{f_{x y}} 1_{x}, \text { para todos } x, y \in G .
$$

Então,

$$
\begin{aligned}
\tilde{f}: G & \longrightarrow \mathcal{Z} \\
x & \longmapsto \widetilde{f}_{x},
\end{aligned}
$$

pertence a $Z_{\Theta}^{1}(G, \alpha, \mathcal{Z})$. Como $\widetilde{f}_{1}=f_{1}(1)=1$ segue que $\tilde{f}$ é um elemento normalizado em $Z_{\Theta}^{1}(G, \alpha, \mathcal{Z})$. Temos o morfismo de grupos

$$
\begin{aligned}
\Psi: \operatorname{Aut}_{R \text {-rings }}(\Delta(\Theta))^{(G)} & \longrightarrow Z_{\Theta}^{1}(G, \alpha, \mathcal{Z}) \\
f & \longmapsto \widetilde{f}
\end{aligned}
$$

Reciprocamente, seja $\sigma \in Z_{\Theta}^{1}(G, \alpha, \mathcal{Z})$ um 1-cociclo normalizado, então

$$
\alpha_{x}\left(\sigma_{y} 1_{y^{-1}}\right) \sigma_{x}=\sigma_{x y} 1_{x}, \quad \text { para todo } x, y \in G .
$$

Para cada $x \in G$ defina

$$
\begin{aligned}
g_{x}: \Theta_{x} & \longrightarrow \Theta_{x} \\
u_{x} & \longmapsto \sigma_{x} u_{x} .
\end{aligned}
$$

Como $\sigma_{x} \in \mathcal{U}\left(\mathcal{Z} 1_{x}\right)$, segue do Lema 3.2.7 que $g_{x}$ é um isomorfismo $R$-bilinear e $\widetilde{g_{x}}=\sigma_{x}$, para todo $x \in G$. Considere

$$
\begin{aligned}
g:=\bigoplus_{x \in G} g_{x}: \Delta(\Theta) & \longrightarrow \Delta(\Theta) \\
u_{x} & \longrightarrow g_{x}\left(u_{x}\right)
\end{aligned} .
$$

Então, $g_{x}\left(\Theta_{x}\right)=\Theta_{x}$, para todo $x \in G$. Como $\sigma$ é normalizado temos

$$
g(r)=g_{1}(r)=\sigma_{1} r=1 r=r, \quad \forall r \in R .
$$

Logo, $g$ fixa cada elemento de $R$. Agora, dados $u_{x} \in \Theta_{x}$ e $u_{y} \in \Theta_{y}$, temos

$$
\begin{aligned}
g\left(u_{x} \stackrel{\ominus}{\circ} u_{y}\right) & =\sigma_{x y}\left(u_{x} \stackrel{\ominus}{\circ} u_{y}\right) \stackrel{(4.2)}{=} \sigma_{x} \alpha_{x}\left(\sigma_{y} 1_{x^{-1}}\right)\left(u_{x} \stackrel{\ominus}{\circ} u_{y}\right) \\
& =\left(\sigma_{x} \alpha_{x}\left(\sigma_{y} 1_{x^{-1}}\right) u_{x} \stackrel{\ominus}{\circ} u_{y}\right) \stackrel{(3.14)}{=}\left(\sigma_{x} u_{x} \sigma_{y} \stackrel{\ominus}{\circ} u_{y}\right) \\
& =\left(\sigma_{x} u_{x} \stackrel{\ominus}{\circ} \sigma_{y} u_{y}\right)=\left(g_{x}\left(u_{x}\right) \stackrel{\ominus}{\circ} g_{y}\left(u_{y}\right)\right) .
\end{aligned}
$$


Portanto, $g \in \mathbf{A u t}_{R \text {-rings }}(\Delta(\Theta))^{(G)}$ e temos a aplicação

$$
\begin{aligned}
\Phi: Z_{\Theta}^{1}(G, \alpha, \mathcal{Z}) & \longrightarrow \text { Aut }_{R \text {-rings }}(\Delta(\Theta) / R)^{(G)} \\
\sigma & \longmapsto g
\end{aligned}
$$

Vejamos que $\Phi$ é a inversa de $\Psi$. Seja $f \in$ Aut $_{R \text {-rings }}(\Delta(\Theta))^{(G)}$, então

$$
\Phi(\Psi(f))=\Phi(\widetilde{f})=\bigoplus_{x \in G} g_{x}
$$

onde $g_{x}\left(u_{x}\right)=\widetilde{f}_{x} u_{x}=f_{x}\left(u_{x}\right)$, para todo $u_{x} \in \Theta_{x}, x \in G$. Logo, $\Psi(\Phi(f))=f$, para todo $f \in$ $\operatorname{Aut}_{R \text {-rings }}(\Delta(\Theta))^{(G)}$.

Por outro lado, seja $\sigma \in Z_{\Theta}^{1}(G, \alpha, \mathcal{Z})$, então

$$
\Psi(\Phi(\sigma))=\Psi(g)=\widetilde{g},
$$

onde $g_{x}\left(u_{x}\right)=\sigma_{x} u_{x}$, para todo $x \in G$. Assim, $\widetilde{g_{x}}=\sigma_{x}$, para todo $x \in G$. Logo $\Psi(\Phi(\sigma))=\sigma$, para todo $\sigma \in Z_{\Theta}^{1}(G, \alpha, \mathcal{Z})$. Portanto, $\Psi: \mathbf{A u t}_{R \text {-rings }}(\Delta(\Theta))^{(G)} \longrightarrow Z_{\Theta}^{1}(G, \alpha, \mathcal{Z})$ é um isomorfismo de grupos.

Afirmação 4.1.6. $\Psi(\operatorname{Im}(\mathcal{F}))=B_{\Theta}^{1}(G, \alpha, \mathcal{Z})$.

De fato, seja $r \in \mathcal{U}(\mathcal{Z})$, então

$$
\mathcal{F}(r)\left(u_{x}\right)=r^{-1} u_{x} r=r^{-1} \alpha_{x}\left(r 1_{x^{-1}}\right) u_{x} \text {, para todo } u_{x} \in \Theta_{x} .
$$

Logo, $\Psi(\mathcal{F}(r))=\delta^{1} r \in B_{\Theta}^{1}(G, \alpha, \mathcal{Z})$. Reciprocamente, se $\sigma \in B_{\Theta}^{1}(G, \alpha, \mathcal{Z})$, então existe um $r \in \mathcal{U}(\mathcal{Z})$ tal que $\delta^{1} r=\sigma$, ou seja,

$$
\sigma(x)=\alpha_{x}\left(r 1_{x^{-1}}\right) r^{-1} .
$$

Então, $\mathcal{F}(r)\left(u_{x}\right)=r^{-1} u_{x} r=r^{-1} \alpha_{x}\left(r 1_{x^{-1}}\right) u_{x}$, para todo $u_{x} \in \Theta_{x}$. Assim, $\widetilde{\mathcal{F}(r)_{x}}=\sigma_{x}$, para todo $x \in G$. Logo, $\Psi(\mathcal{F}(r))=\sigma$. O que mostra a afirmação.

Temos então o isomorfismo

$$
\frac{\text { Aut }_{R \text {-rings }}(\Delta(\Theta))^{(G)}}{\operatorname{Im}(\mathcal{F})} \simeq H_{\Theta}^{1}(G, \alpha,, \mathcal{Z}) .
$$

\subsection{A segunda sequência exata}

Seja

$$
\operatorname{Pic}_{\mathcal{Z}}(R)^{(G)}=\left\{[P] \in \mathbf{P i c}_{\mathcal{Z}}(R) ; P \otimes \Theta_{x} \otimes P^{-1} \mid \Theta_{x}, \text { para todo } x \in G\right\} .
$$

Lema 4.2.1. $\mathbf{P i c}_{\mathcal{Z}}(R)^{(G)}$ é um subgrupo de $\mathbf{P i c}_{\mathcal{Z}}(R)$ que contém $\mathbf{P i c}_{\mathcal{Z}}(R) \cap \mathbf{P i c S}_{\mathcal{Z}}(R)^{\alpha^{*}}$.

Demonstração. Sejam $[P],[Q] \in \mathbf{P i c}_{\mathcal{Z}}(R)^{(G)}$. Como $Q \otimes \Theta_{x} \otimes Q^{-1} \mid \Theta_{x}$, segue da compatibilidade com o produto tensorial que $P \otimes Q \otimes \Theta_{x} \otimes Q^{-1} \otimes_{R} P^{-1} \mid P \otimes \Theta_{x} \otimes P^{-1}$. Portanto, pela transitividade, temos que

$$
P \otimes Q \otimes \Theta_{x} \otimes Q^{-1} \otimes P^{-1} \mid \Theta_{x}, \quad \text { para todo } x \in G .
$$


Logo, $[P \otimes Q] \in \mathbf{P i c}_{\mathcal{Z}}(R)^{(G)}$. Vejamos que $\mathbf{P i c}_{\mathcal{Z}}(R)^{(G)}$ também é fechado para inverso. Seja $[P] \in$ $\operatorname{Pic}_{\mathcal{Z}}(R)^{(G)}$. Temos

$$
\begin{aligned}
P^{-1} \otimes \Theta_{x} \otimes P & \simeq P^{-1} \otimes \Theta_{x} \otimes R 1_{x^{-1}} \otimes P \simeq P^{-1} \otimes \Theta_{x} \otimes P \otimes R 1_{x^{-1}} \\
& \simeq P^{-1} \otimes \Theta_{x} \otimes P \otimes \Theta_{x^{-1}} \otimes \Theta_{x} \simeq P^{-1} \otimes \Theta_{x} \otimes P \otimes \Theta_{x^{-1}} \otimes R \otimes \Theta_{x} \\
& \simeq P^{-1} \otimes \Theta_{x} \otimes P \otimes \Theta_{x^{-1}} \otimes P^{-1} \otimes P \otimes \Theta_{x} .
\end{aligned}
$$

Assim, como $P \otimes \Theta_{x} \otimes P^{-1} \mid \Theta_{x}$, para todo $x \in G$, temos

$$
P^{-1} \otimes \Theta_{x} \otimes P \otimes \Theta_{x^{-1}} \otimes P^{-1} \otimes P \otimes \Theta_{x} \mid P^{-1} \otimes \Theta_{x} \otimes \Theta_{x^{-1}} \otimes P \otimes \Theta_{x} .
$$

Porém,

$$
P^{-1} \otimes \Theta_{x} \otimes \Theta_{x^{-1}} \otimes P \otimes \Theta_{x} \simeq P^{-1} \otimes R 1_{x} \otimes P \otimes \Theta_{x} \simeq P^{-1} \otimes P \otimes R 1_{x} \otimes \Theta_{x} \simeq \Theta_{x} .
$$

Logo, $P^{-1} \otimes \Theta_{x} \otimes P \mid \Theta_{x}$, para todo $x \in G$. Assim, $\left[P^{-1}\right] \in \mathbf{P i c}_{\mathcal{Z}}(R)^{(G)}$. Portanto, $\mathbf{P i c}_{\mathcal{Z}}(R)^{(G)}$ é um subgrupo de $\mathbf{P i c}_{\mathcal{Z}}(R)$.

Seja $[P] \in \mathbf{P i c}_{\mathcal{Z}}(R) \cap \operatorname{PicS}_{\mathcal{Z}}(R)^{\alpha^{*}}$. Por (3.18) temos que $\Theta_{x} \otimes P \simeq P \otimes \Theta_{x}$, para todo $x \in G$. Com $[P] \in \operatorname{Pic}_{\mathcal{Z}}(R)$, segue que

$$
P \otimes \Theta_{x} \otimes P^{-1} \simeq \Theta_{x}, \forall x \in G,[P] \in \operatorname{Pic}_{\mathcal{Z}}(R) \cap \operatorname{PicS}_{\mathcal{Z}}(R)^{\alpha^{*}} .
$$

Em particular, $P \otimes \Theta_{x} \otimes P^{-1} \mid \Theta_{x}$, para todo $x \in G$. Portanto, $\mathbf{P i c}_{\mathcal{Z}}(R) \cap \mathbf{P i c S}_{\mathcal{Z}}(R)^{\alpha^{*}} \subseteq \mathbf{P i c}_{\mathcal{Z}}(R)^{(G)}$.

Nosso objetivo é construir um produto cruzado generalizado parcial a partir de um elemento $[P] \in$ $\operatorname{Pic}_{\mathcal{Z}}(R)^{(G)}$. Vamos começar construindo uma representação parcial unital.

Lema 4.2.2. Seja $[P] \in \mathbf{P i c}_{\mathcal{Z}}(R)^{(G)}$ e denote $\Omega_{x}^{P}=P \otimes \Theta_{x} \otimes P^{-1}$, para todo $x \in G$. Então

$$
\begin{aligned}
\Omega^{P}: \quad G & \longrightarrow \operatorname{PicS}(R) \\
x & \longmapsto\left[\Omega_{x}^{P}\right] .
\end{aligned}
$$

é uma representação parcial unital com $\Omega_{x}^{P} \otimes \Omega_{x^{-1}}^{P} \simeq R 1_{x}$ e $\Omega_{x}^{P} \mid \Theta_{x}$, para todo $x \in G$.

Demonstração. Claramente $\left[\Omega_{1}^{P}\right]=[R]$. Dados $x, y \in G$ temos

$$
\begin{aligned}
\Omega_{x}^{P} \otimes \Omega_{y}^{P} \otimes \Omega_{y^{-1}}^{P} & =P \otimes \Theta_{x} \otimes P^{-1} \otimes P \otimes \Theta_{y} \otimes P^{-1} \otimes P \otimes \Theta_{y^{-1}} \otimes P^{-1} \\
& \simeq P \otimes \Theta_{x} \otimes \Theta_{y} \otimes \Theta_{y^{-1}} \otimes P^{-1} \\
& \simeq P \otimes \Theta_{x y} \otimes \Theta_{y^{-1}} \otimes P^{-1} \\
& \simeq P \otimes \Theta_{x y} \otimes P^{-1} \otimes P \otimes \Theta_{y^{-1}} \otimes P^{-1} \\
& =\Omega_{x y}^{P} \otimes \Omega_{y^{-1}}^{P} .
\end{aligned}
$$

Analogamente, temos que $\Omega_{x^{-1}}^{P} \otimes \Omega_{x}^{P} \otimes \Omega_{y}^{P} \simeq \Omega_{x^{-1}}^{P} \otimes \Omega_{x y}^{P}$, para todo $x, y \in G$.

Além disso, temos

$$
\begin{aligned}
\Omega_{x}^{P} \otimes \Omega_{x^{-1}}^{P} & =P \otimes \Theta_{x} \otimes P^{-1} \otimes P \otimes \Theta_{x^{-1}} \otimes P^{-1} \\
& \simeq P \otimes \Theta_{x} \otimes \Theta_{x^{-1}} \otimes P^{-1} \\
& \simeq P \otimes R 1_{x} \otimes P^{-1} \\
& \simeq R 1_{x} \otimes P \otimes P^{-1} \\
& \simeq R 1_{x} \otimes R \simeq R 1_{x}, \quad \forall x \in G .
\end{aligned}
$$


Portanto, $\Omega^{P}$ é uma representação parcial unital com $\Omega_{x}^{P} \otimes \Omega_{x^{-1}}^{P} \simeq R 1_{x}$, para todo $x \in G$.

Dado $[P] \in \mathbf{P i c}_{\mathcal{Z}}(R)^{(G)}$ denote $P^{-1} \otimes P \stackrel{\mathfrak{r}}{\longrightarrow} R \stackrel{\mathfrak{l}}{\longleftarrow} P \otimes P^{-1}$ isomorfismo de $R$-bimódulos. Defina os isomorfismos de $R$-bimódulos $f_{x, y}^{P}: \Omega_{x}^{P} \otimes \Omega_{y}^{P} \longrightarrow 1_{x} \Omega_{x y}^{P}$ via

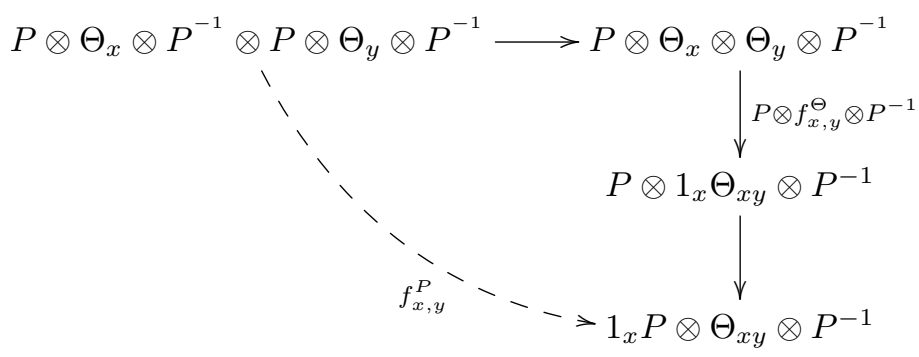

onde o primeiro isomorfismo é induzido por $\mathfrak{r}$, ou seja,

$$
f_{x, y}^{P}\left(p_{1} \otimes u_{x} \otimes \bar{p}_{1} \otimes p_{2} \otimes u_{y} \otimes \bar{p}_{2}\right)=p_{1} \otimes\left(u_{x} \mathfrak{r}\left(\bar{p}_{1} \otimes p_{2}\right) \stackrel{\ominus}{\circ} u_{y}\right) \otimes \bar{p}_{2},
$$

para $u_{x} \in \Theta_{x}, u_{y} \in \Theta_{y}, p_{1}, p_{2} \in P$ e $\bar{p}_{1}, \bar{p}_{2} \in P^{-1}$.

Afirmação 4.2.3. $f^{P}=\left\{f_{x, y}^{P}: \Omega_{x}^{P} \otimes \Omega_{y}^{P} \longrightarrow 1_{x} \Omega_{x y}^{P}, x, y \in G\right\}$ é um conjunto de fatores para $\Omega^{P}$.

De fato, dados $x, y, z \in G$, vejamos que o diagrama abaixo é comutativo

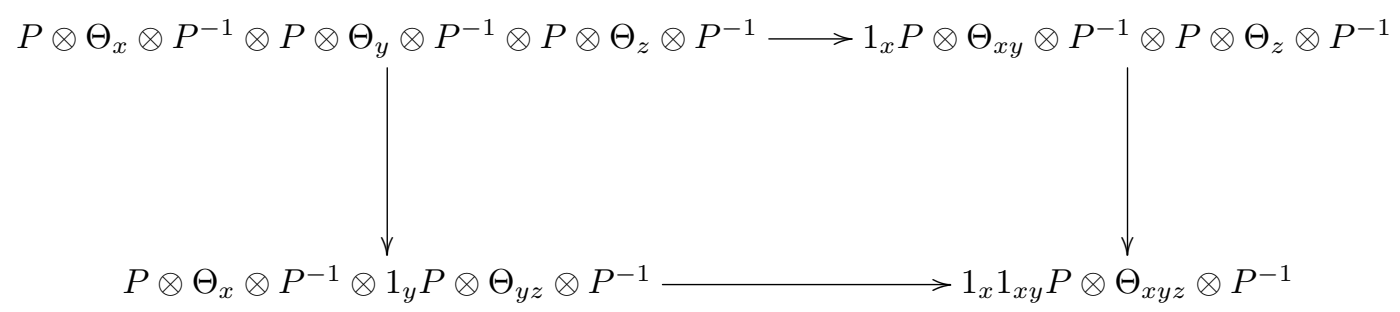

Considere $u_{x} \in \Theta_{x}, u_{y} \in \Theta_{y}, u_{z} \in \Theta_{z}, p_{1}, p_{2}, p_{3} \in P$ e $\bar{p}_{1}, \bar{p}_{2}, \bar{p}_{3} \in P^{-1}$, então

$$
\begin{aligned}
& {\left[f_{x y, z}^{P} \circ\left(f_{x, y}^{P} \otimes \Omega_{z}^{P}\right)\right]\left(p_{1} \otimes u_{x} \otimes \bar{p}_{1} \otimes p_{2} \otimes u_{y} \otimes \bar{p}_{2} \otimes p_{3} \otimes u_{z} \otimes \bar{p}_{3}\right) } \\
= & f_{x y, z}^{P}\left(p_{1} \otimes\left(u_{x} \mathfrak{r}\left(\bar{p}_{1} \otimes p_{2}\right) \stackrel{\ominus}{\circ} u_{y}\right) \otimes \bar{p}_{2} \otimes p_{3} \otimes u_{z} \otimes \bar{p}_{3}\right) \\
= & p_{1} \otimes\left(\left(u_{x} \mathfrak{r}\left(\bar{p}_{1} \otimes p_{2}\right) \stackrel{\ominus}{\circ} u_{y}\right) \mathfrak{r}\left(\bar{p}_{2} \otimes p_{3}\right) \stackrel{\ominus}{\circ} u_{z}\right) \otimes \bar{p}_{3} \\
= & p_{1} \otimes\left(u_{x} \mathfrak{r}\left(\bar{p}_{1} \otimes p_{2}\right) \stackrel{\ominus}{\circ}\left(u_{y} \mathfrak{r}\left(\bar{p}_{2} \otimes p_{3}\right) \stackrel{\ominus}{\circ} u_{z}\right)\right) \otimes \bar{p}_{3} \\
= & f_{x, y z}^{P}\left(p_{1} \otimes u_{x} \otimes \bar{p}_{1} \otimes p_{2} \otimes\left(u_{y} \mathfrak{r}\left(\bar{p}_{2} \otimes p_{3}\right) \stackrel{\ominus}{\circ} u_{z}\right) \otimes \bar{p}_{3}\right) \\
= & {\left[f_{x, y z}^{P} \circ\left(\Omega_{x}^{P} \otimes f_{y, z}^{P}\right)\right]\left(p_{1} \otimes u_{x} \otimes \bar{p}_{1} \otimes p_{2} \otimes u_{y} \otimes \bar{p}_{2} \otimes p_{3} \otimes u_{z} \otimes \bar{p}_{3}\right) . }
\end{aligned}
$$

Logo, $f_{x y, z}^{P} \circ\left(f_{x, y}^{P} \otimes \Omega_{z}^{P}\right)=f_{x, y z}^{P} \circ\left(\Omega_{x}^{P} \otimes f_{y, z}^{P}\right)$ o que mostra a comutatividade do diagrama acima. Portanto, $f^{P}=\left\{f_{x, y}^{P}: \Omega_{x}^{P} \otimes \Omega_{y}^{P} \longrightarrow 1_{x} \Omega_{x y}^{P}, x, y \in G\right\}$ é um conjunto de fatores para $\Omega^{P}$.

Temos então um produto cruzado generalizado parcial

$$
\Delta\left(\Omega^{P}\right)=\bigoplus_{x \in G} P \otimes \Theta_{x} \otimes P^{-1},
$$

tal que $P \otimes \Theta_{x} \otimes P^{-1} \mid \Theta_{x}$ e $\Omega_{x}^{P} \otimes \Omega_{x^{-1}}^{P} \simeq R 1_{x}$, para todo $x \in G$. Logo, $\left[\Delta\left(\Omega^{P}\right)\right] \in \mathcal{C}(\Theta / R)$, para todo $[P] \in \mathbf{P i c}_{\mathcal{Z}}(R)^{(G)}$. 
Observação 4.2.4. É importante observar que a classe de isomorfismo de $\Delta\left(\Omega^{P}\right)$ em $\mathcal{C}(\Theta / R)$ não depende da escolha do isomorfismo $\mathfrak{r}$ usado para definir os isomorfismo $f_{x, y}^{P}$ no conjunto de fatores de $\Omega^{P}$. De fato, vamos considerar um novo isomorfismo de $R$-bimódulos $\widetilde{\mathfrak{r}}: P \otimes_{R} P^{-1} \longrightarrow R$, e denote $\widetilde{f}_{x, y}^{P}$ o isomorfismo definido a partir de $\widetilde{\mathfrak{r}}$ e $\Delta\left(\widetilde{\Omega}^{P}\right)$ o produto cruzado generalizado parcial com conjunto de fatores $\widetilde{f}^{P}$. Como $\widetilde{\mathfrak{r}} \circ \mathfrak{r}^{-1} \in \mathbf{A u t}_{R-R}(R) \simeq \mathcal{U}(\mathcal{Z})$, então existe $a \in \mathcal{U}(\mathcal{Z})$ tal que $\widetilde{\mathfrak{r}}=$ ar. Considere o isomorfismo de R-bimódulos

$$
\begin{aligned}
\varphi_{x}: \quad P \otimes \Theta_{x} \otimes P^{-1} & \longrightarrow P \otimes \Theta_{x} \otimes P^{-1} \\
p \otimes u_{x} \otimes p^{-1} & \longmapsto p \otimes a^{-1} u_{x} \otimes p^{-1} .
\end{aligned}
$$

Então o diagrama abaixo é comutativo

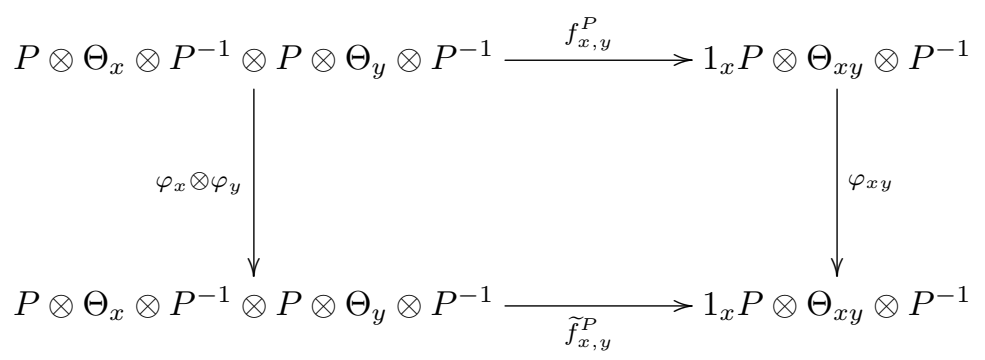

De fato, sejam $p_{1}, p_{2} \in P, \bar{p}_{1}, \bar{p}_{2} \in P^{-1}, u_{x} \in \Theta_{x}$ e $u_{y} \in \Theta_{y}$. Por um lado,

$$
\varphi_{x y} \circ f_{x, y}^{P}\left(p_{1} \otimes u_{x} \otimes \bar{p}_{1} \otimes p_{2} \otimes u_{y} \otimes \bar{p}_{2}\right)=p_{1} \otimes\left(a^{-1} u_{x} \mathfrak{r}\left(\bar{p}_{1} \otimes p_{2}\right) \stackrel{\ominus}{\circ} u_{y}\right) \otimes \bar{p}_{2} .
$$

Por outro lado,

$$
\begin{aligned}
\widetilde{f}_{x, y}^{P} \circ\left(\varphi_{x} \otimes \varphi_{y}\right)\left(p_{1} \otimes u_{x} \otimes \bar{p}_{1} \otimes p_{2} \otimes u_{y} \otimes \bar{p}_{2}\right) & =\widetilde{f}_{x, y}^{P}\left(p_{1} \otimes a^{-1} u_{x} \otimes \bar{p}_{1} \otimes p_{2} \otimes a^{-1} u_{y} \otimes \bar{p}_{2}\right) \\
& =p_{1} \otimes\left(a^{-1} u_{x} \widetilde{\mathfrak{r}}\left(\bar{p}_{1} \otimes p_{2}\right) \stackrel{\ominus}{\circ} a^{-1} u_{y}\right) \otimes \bar{p}_{2} \\
& =p_{1} \otimes\left(a^{-1} u_{x} a \mathfrak{r}\left(\bar{p}_{1} \otimes p_{2}\right) \stackrel{\ominus}{\circ} a^{-1} u_{y}\right) \otimes \bar{p}_{2} \\
& =p_{1} \otimes\left(a^{-1} u_{x} \mathfrak{r}\left(\bar{p}_{1} \otimes p_{2}\right) \stackrel{\ominus}{\circ} a a^{-1} u_{y}\right) \otimes \bar{p}_{2} \\
& =p_{1} \otimes\left(a^{-1} u_{x} \mathfrak{r}\left(\bar{p}_{1} \otimes p_{2}\right) \stackrel{\ominus}{\circ} u_{y}\right) \otimes \bar{p}_{2} .
\end{aligned}
$$

Logo, o diagrama é comutativo. Portanto, $\left[\Delta\left(\Omega^{P}\right)\right]=\left[\Delta\left(\widetilde{\Omega}^{P}\right)\right]$ em $\mathcal{C}(\Theta / R)$.

Sendo $\Delta\left(\Omega^{P}\right)$ um produto cruzado generalizado parcial, pela Observação 3.3.4, existe um isomorfismo $\nu_{P}: R \longrightarrow P \otimes \Theta_{1} \otimes P^{-1}$ que satisfaz os diagramas comutativos como em (3.23). Na próxima observação vamos definir esse isomorfismo. Ele será usado na demonstração de resultados mais a diante.

Observação 4.2.5. Seja $\iota: R \longrightarrow \Theta_{1}$ o isomorfismo de R-bimódulos que satisfaz os diagramas comutativos em (3.23). Considere $\nu_{P}: R \longrightarrow P \otimes \Theta_{1} \otimes P^{-1}$ o isomorfismo definido por

$$
\nu_{P}(r)=\sum_{k=1}^{n} r p_{k} \otimes \iota(1) \otimes \bar{p}_{k}
$$

onde $\sum_{k=1}^{n} \mathfrak{r}\left(p_{k} \otimes \bar{p}_{k}\right)=1$. Então, $\nu_{P}$ satisfaz os diagramas comutativos
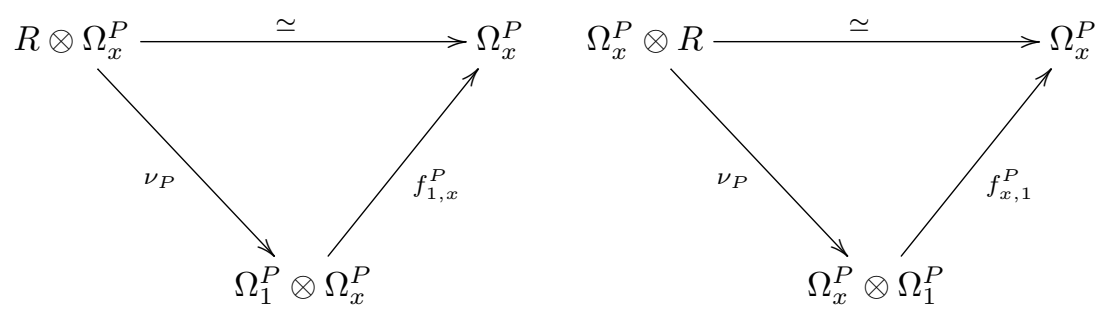
De fato, dados $r \in R, p \in P, u_{x} \in \Theta_{x}$ e $\bar{p} \in P^{-1}$ temos

$$
\begin{aligned}
f_{1, x}^{P}\left(\nu_{P}(r) \otimes p \otimes u_{x} \otimes \bar{p}\right) & =\sum_{k=1}^{n} f_{1, x}^{P}\left(r p_{k} \otimes \iota(1) \otimes \bar{p}_{k} \otimes p \otimes u_{x} \otimes \bar{p}\right) \\
& =\sum_{i=1}^{n} r p_{k} \otimes f_{1, x}^{\Theta}\left(\iota(1) \otimes \mathfrak{r}\left(\bar{p}_{k} \otimes p\right) u_{x}\right) \otimes \bar{p} \\
& =\sum_{i=1}^{n} r p_{k} \otimes \mathfrak{r}\left(\bar{p}_{k} \otimes p\right) u_{x} \otimes \bar{p} \\
& =\sum_{i=1}^{n} r p_{k} \mathfrak{r}\left(\bar{p}_{k} \otimes p\right) \otimes u_{x} \otimes \bar{p} \\
& =\sum_{i=1}^{n} r \mathfrak{l}\left(p_{k} \otimes \bar{p}_{k}\right) p \otimes u_{x} \otimes \bar{p} \\
& =r p \otimes u_{x} \otimes \bar{p} .
\end{aligned}
$$

A comutatividade do segundo diagrama é análogo.

Teorema 4.2.6. A aplicação

$$
\begin{aligned}
\mathcal{L}: \operatorname{Pic}_{\mathcal{Z}}(R)^{(G)} & \longrightarrow \mathcal{C}(\Theta / R) \\
{[P] } & \longmapsto
\end{aligned}
$$

é um morfismo de grupos.

Demonstração. Vejamos primeiro que $\mathcal{L}$ está bem definida. Seja $[P]=[Q]$ em $\mathbf{P i c}_{\mathcal{Z}}(R)^{(G)}$, então existem isomorfismos de $R$-bimódulos $\varphi: Q \longrightarrow P$ e $\bar{\varphi}: Q^{-1} \longrightarrow P^{-1}$. Seja $\mathfrak{r}: P^{-1} \otimes P \longrightarrow R$ um isomorfismo de $R$-bimódulos, vamos considerar o isomorfismo de $R$-bimódulos $\mathfrak{r}^{\prime}: Q^{-1} \otimes Q \longrightarrow R$ definido por $\mathfrak{r}^{\prime}(\bar{q} \otimes q)=\mathfrak{r}(\bar{\varphi}(\bar{q}) \otimes \varphi(q))$.

Para cada $x \in G$ considere o isomorfismo de $R$-bimódulos

$$
\begin{aligned}
\varphi_{x}: \quad Q \otimes \Theta_{x} \otimes Q^{-1} & \longrightarrow P \otimes \Theta_{x} \otimes P^{-1} \\
q \otimes u_{x} \otimes \bar{q} & \longmapsto \varphi(q) \otimes u_{x} \otimes \bar{\varphi}(\bar{q}) .
\end{aligned}
$$

Então, o diagrama

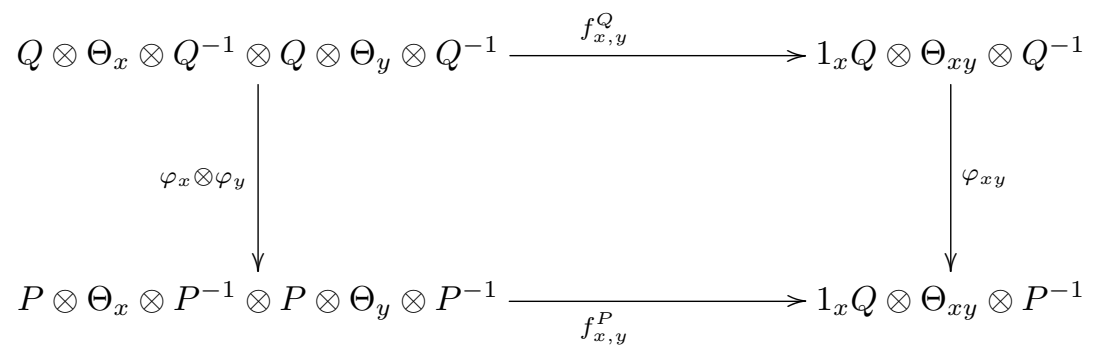

é comutativo. De fato, sejam $u_{x} \in \Theta_{x}, u_{y} \in \Theta_{y}, q_{1}, q_{2} \in Q$ e $\bar{q}_{1}, \bar{q}_{2} \in Q^{-1}$, então

$$
\begin{aligned}
\left(\varphi_{x y} \circ f_{x, y}^{Q}\right)\left(q_{1} \otimes u_{x} \otimes \bar{q}_{1} \otimes q_{2} \otimes u_{y} \otimes \bar{q}_{2}\right) & =\varphi_{x y}\left(q_{1} \otimes\left(u_{x} \mathfrak{r}^{\prime}\left(\bar{q}_{1} \otimes q_{2}\right) \stackrel{\ominus}{\circ} u_{y}\right) \otimes \bar{q}_{2}\right) \\
& =\varphi_{x y}\left(q_{1} \otimes\left(u_{x} \mathfrak{r}\left(\bar{\varphi}\left(\bar{q}_{1}\right) \otimes \varphi\left(q_{2}\right)\right) \stackrel{\ominus}{\circ} u_{y}\right) \otimes \bar{q}_{2}\right) \\
& =\varphi\left(q_{1}\right) \otimes\left(u_{x} \mathfrak{r}\left(\bar{\varphi}\left(\bar{q}_{1}\right) \otimes \varphi\left(q_{2}\right)\right) \stackrel{\ominus}{\circ} u_{y}\right) \otimes \bar{\varphi}\left(\bar{q}_{2}\right) \\
& =f_{x, y}^{P}\left(\varphi\left(q_{1}\right) \otimes u_{x} \otimes \bar{\varphi}\left(\bar{q}_{1}\right) \otimes \varphi\left(q_{2}\right) \otimes u_{y} \otimes \bar{\varphi}\left(\bar{q}_{2}\right)\right) \\
& =\left(f_{x, y}^{P} \circ\left(\varphi_{x} \otimes \varphi_{y}\right)\right)\left(q_{1} \otimes u_{x} \otimes \bar{q}_{1} \otimes q_{2} \otimes u_{y} \otimes \bar{q}_{2}\right) .
\end{aligned}
$$


Logo, $\left[\Delta\left(\Omega^{P}\right)\right]=\left[\Delta\left(\Omega^{Q}\right)\right]$ em $\mathcal{C}(\Theta / R)$ e portanto $\mathcal{L}$ está bem definida.

Sejam $[P],[Q] \in \mathbf{P i c}_{\mathcal{Z}}(R)^{(G)}$. Vamos considerar os isomorfismos de $R$-bimódulos

$$
P \otimes P^{-1} \stackrel{\mathfrak{l}}{\longrightarrow} R \stackrel{\mathfrak{r}}{\longleftarrow} P^{-1} \otimes P \quad \text { e } \quad Q \otimes Q^{-1} \stackrel{\mathfrak{l}^{\prime}}{\longrightarrow} R \stackrel{\mathfrak{r}^{\prime}}{\longleftarrow} Q^{-1} \otimes Q .
$$

Então,

$$
\mathcal{L}([P \otimes Q])=\left[\bigoplus_{x \in G} P \otimes Q \otimes \Theta_{x} \otimes Q^{-1} \otimes P^{-1}\right]
$$

Por outro lado,

$$
\mathcal{L}([P]) \mathcal{L}([Q])=\left[\bigoplus_{x \in G} P \otimes \Theta_{x} \otimes P^{-1} \otimes \Theta_{x^{-1}} \otimes Q \otimes \Theta_{x} \otimes Q^{-1}\right] .
$$

Como $Q \otimes \Theta_{x} \otimes Q^{-1} \mid \Theta_{x}$, então $\Theta_{x^{-1}} \otimes Q \otimes \Theta_{x} \otimes Q^{-1} \mid R$. Por $P^{-1}$ ser um $\mathcal{Z}$-bimódulos central, segue do Corolário 2.3.6, que existe um isomorfismo de $R$-bimódulos

$$
T: P^{-1} \otimes \Theta_{x^{-1}} \otimes Q \otimes \Theta_{x} \otimes Q^{-1} \longrightarrow \Theta_{x^{-1}} \otimes Q \otimes \Theta_{x} \otimes Q^{-1} \otimes P^{-1} .
$$

Analogamente, por $Q$ ser $\mathcal{Z}$-bimódulo central e $R 1_{x} \mid R$, temos o isomorfismo de $R$-bimódulos

$$
T^{\prime}: R 1_{x} \otimes Q \longrightarrow Q \otimes R 1_{x}, \quad \text { para todo } \quad x \in G .
$$

Vamos definir o isomorfismo de $R$-bimódulo $F_{x}: \Omega_{x}^{P} \otimes \Theta_{x^{-1}} \otimes \Omega_{x}^{Q} \longrightarrow \Omega_{x}^{P \otimes Q}$ via:

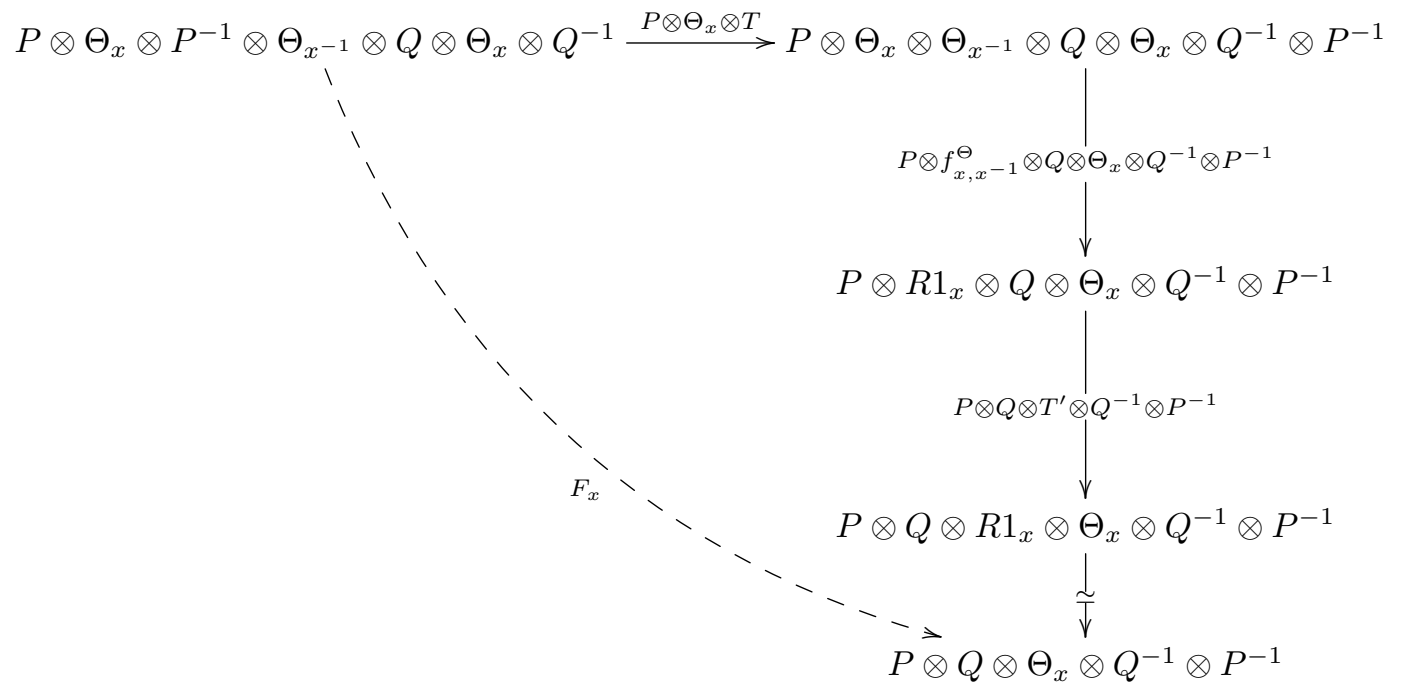

Vamos verificar que o diagrama abaixo é comutativo

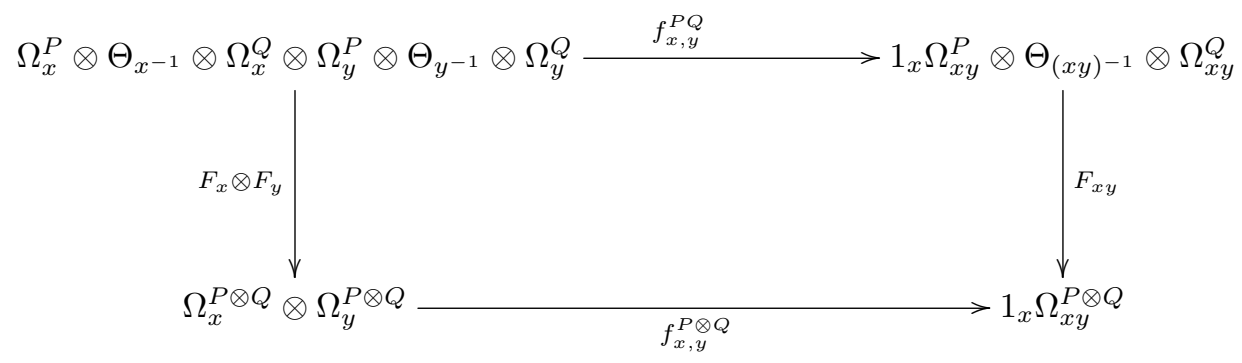


Por um lado, temos que $F_{x y} \circ f_{x, y}^{P Q}$ é dado pelal sequência de isomorfismos

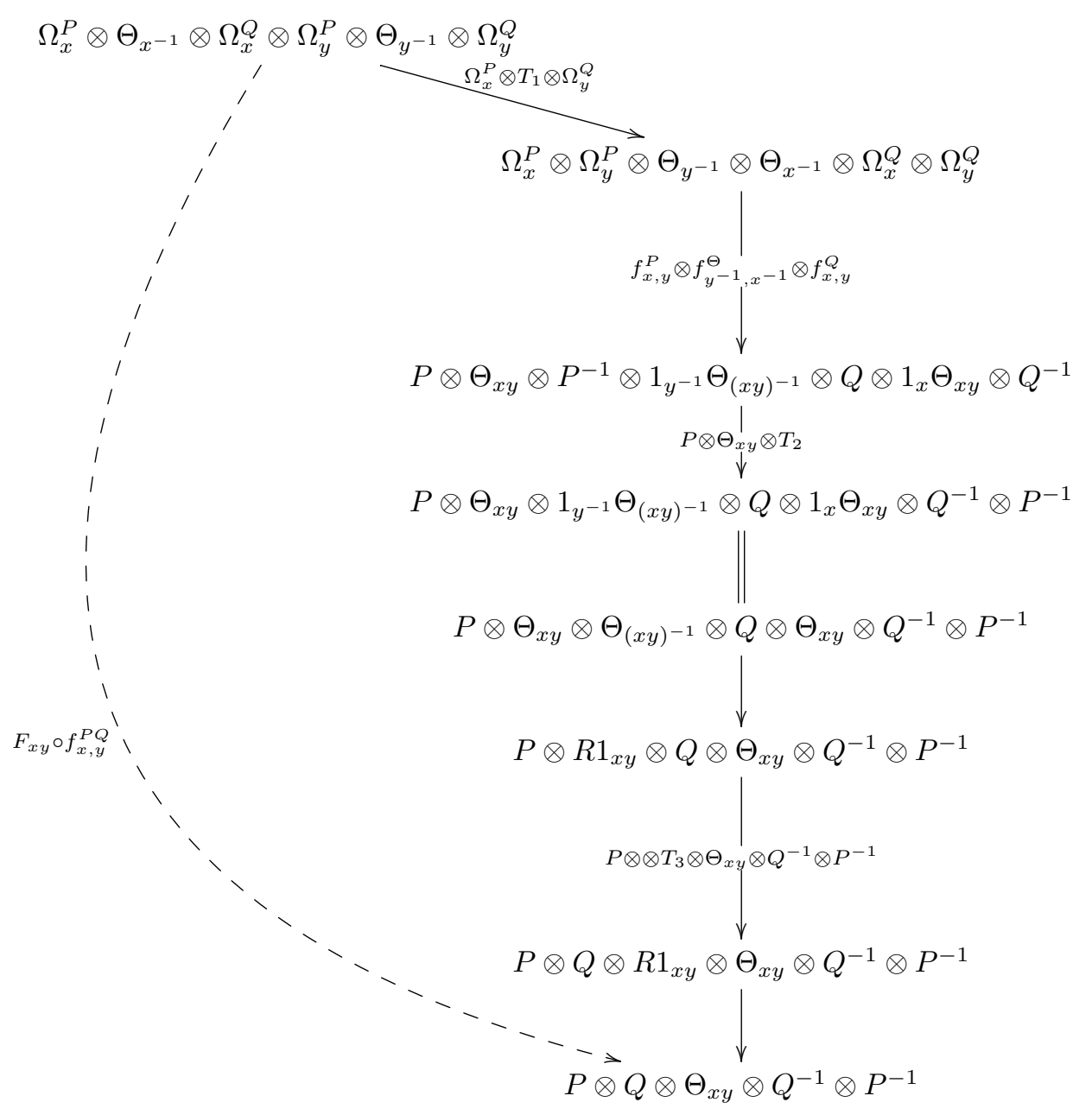

onde,

$T_{1}: \Theta_{x^{-1}} \otimes Q \otimes \Theta_{x} \otimes Q^{-1} \otimes P \otimes \Theta_{y} \otimes P^{-1} \otimes \Theta_{y^{-1}} \longrightarrow P \otimes \Theta_{y} \otimes P^{-1} \otimes \Theta_{y^{-1}} \otimes \Theta_{x^{-1}} \otimes Q \otimes \Theta_{x} \otimes Q^{-1}$ $T_{2}: P^{-1} \otimes 1_{y^{-1}} \Theta_{(x y)^{-1}} \otimes Q \otimes 1_{x} \Theta_{x y} \otimes Q^{-1} \longrightarrow \Theta_{(x y)^{-1}} \otimes Q \otimes \Theta_{x y} \otimes Q^{-1} \otimes P^{-1}$

e

$$
T_{3}: R 1_{x y} \otimes Q \longrightarrow Q \otimes R 1_{x y}
$$

Por outro lado, $f_{x, y}^{P \otimes Q} \circ\left(F_{x} \otimes F_{y}\right)$ é dado por: Sejam

$$
\begin{aligned}
& T_{4}: P^{-1} \otimes \Theta_{x^{-1}} \otimes Q \otimes \Theta_{x} \otimes Q^{-1} \longrightarrow \Theta_{x^{-1}} \otimes Q \otimes \Theta_{x} \otimes Q^{-1} \otimes P^{-1} \\
& T_{5}: P^{-1} \otimes \Theta_{y^{-1}} \otimes Q \otimes \Theta_{y} \otimes Q^{-1} \longrightarrow \Theta_{y^{-1}} \otimes Q \otimes \Theta_{y} \otimes Q^{-1} \otimes P^{-1}
\end{aligned}
$$

e

$$
T_{6}: R 1_{x} \otimes Q \longrightarrow Q \otimes R 1_{x}, \quad T_{7}: R 1_{y} \otimes Q \longrightarrow Q \otimes R 1_{y}
$$

Então 


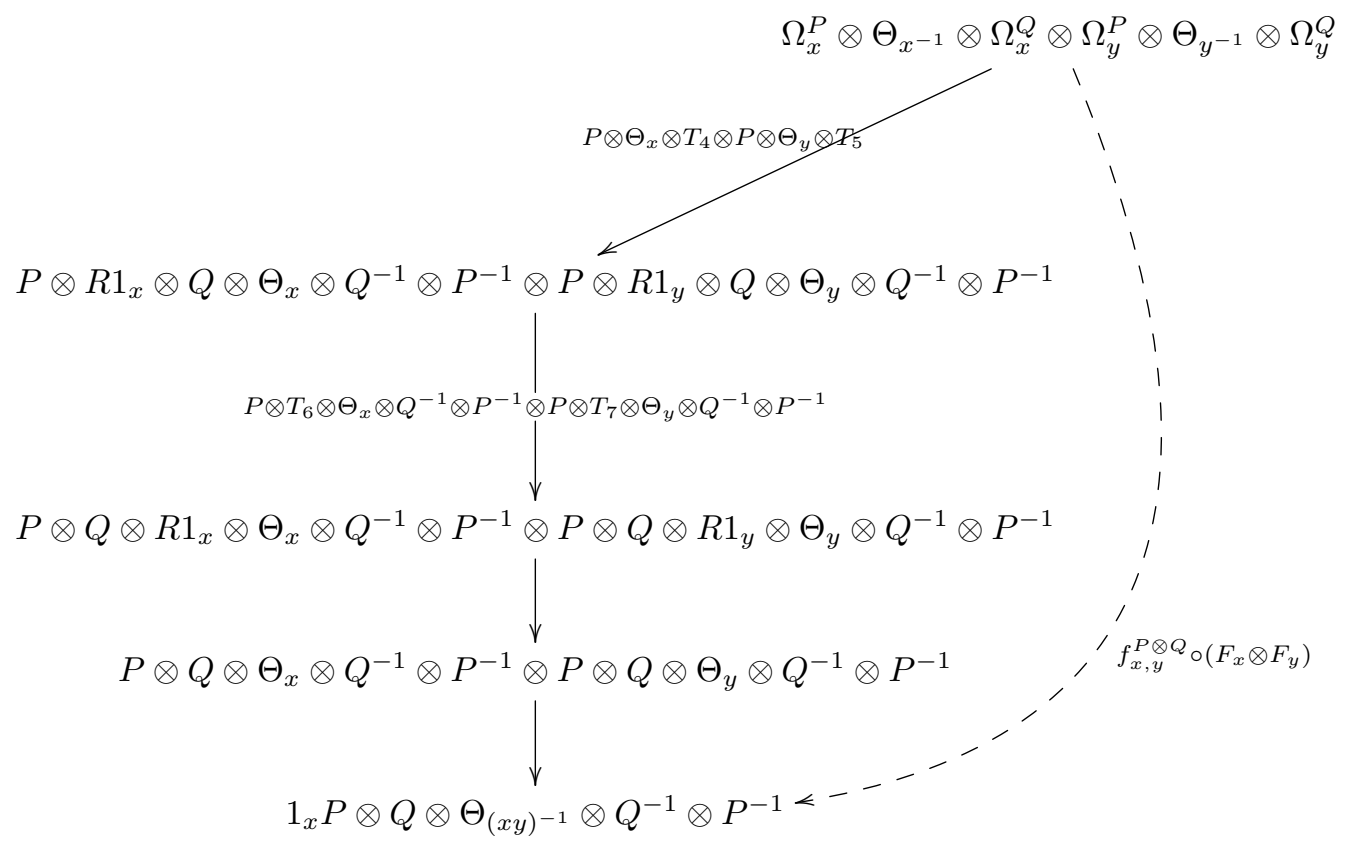

Vamos construir os isomorfismos: Considere as aplicações $R$-bilineares

$$
\begin{aligned}
& f_{i}^{x}: \Theta_{x^{-1}} \otimes Q \otimes \Theta_{x} \otimes Q^{-1} \longrightarrow R \text { e } g_{i}^{x}: R \longrightarrow \Theta_{x^{-1}} \otimes Q \otimes \Theta_{x} \otimes Q^{-1}, \text { com } i=1,2, \ldots, n \\
& f_{j}^{y}: \Theta_{y^{-1}} \otimes Q \otimes \Theta_{y} \otimes Q^{-1} \longrightarrow R \text { e } g_{j}^{y}: R \longrightarrow \Theta_{y^{-1}} \otimes Q \otimes \Theta_{y} \otimes Q^{-1}, \text { com } j=1,2, \ldots, m,
\end{aligned}
$$

tais que $\sum_{i=1}^{n} g_{i}^{x} f_{i}^{x}=I d_{\Theta_{x^{-1}} \otimes Q \otimes \Theta_{x} \otimes Q^{-1}}$ e $\sum_{j=1}^{m} g_{j}^{y} f_{j}^{y}=I d_{\Theta_{y^{-1}} \otimes Q \otimes \Theta_{y} \otimes Q^{-1}}$. Denote,

$$
g_{i}^{x}(1)=\sum_{l} u_{x^{-1}}^{i, l} \otimes q_{x}^{i, l} \otimes u_{x}^{i, l} \otimes q_{x^{-1}}^{i, l} \text { e } g_{j}^{y}(1)=\sum_{k} \widetilde{u}_{y^{-1}}^{j, k} \otimes \widetilde{q}_{y}^{j, k} \otimes \widetilde{u}_{y}^{j, k} \otimes \widetilde{q}_{y^{-1}}^{j, k} .
$$

Então,

$$
\begin{aligned}
T_{4}: \quad P^{-1} \otimes \Theta_{x^{-1}} \otimes Q \otimes \Theta_{x} \otimes Q^{-1} & \longrightarrow \Theta_{x^{-1}} \otimes Q \otimes \Theta_{x} \otimes Q^{-1} \otimes P^{-1} \\
\bar{p} \otimes u_{x^{-1}} \otimes q \otimes u_{x} \otimes \bar{q} & \longmapsto \sum u_{x^{-1}}^{i, l} \otimes q_{x}^{i, l} \otimes u_{x}^{i, l} \otimes q_{x^{-1}}^{i, l} \otimes \bar{p} f_{i}^{x}\left(u_{x^{-1}} \otimes q \otimes u_{x} \otimes \bar{q}\right)
\end{aligned}
$$

$$
\begin{aligned}
& T_{5}: \quad P^{-1} \otimes \Theta_{y^{-1}} \otimes Q \otimes \Theta_{y} \otimes Q^{-1} \longrightarrow \Theta_{y^{-1}} \otimes Q \otimes \Theta_{y} \otimes Q^{-1} \otimes P^{-1} \\
& \bar{p} \otimes u_{y^{-1}} \otimes q \otimes u_{y} \otimes \bar{q} \quad \longmapsto \sum \sum_{y^{-1}}^{j, k} \otimes \widetilde{q}_{y}^{j, k} \otimes \widetilde{u}_{y}^{j, k} \otimes \widetilde{q}_{y^{-1}}^{j, k} \otimes \bar{p} f_{j}^{y}\left(u_{y^{-1}} \otimes q \otimes u_{y} \otimes \bar{q}\right),
\end{aligned}
$$

e

$T_{1}: \quad \Theta_{x^{-1}} \otimes Q \otimes \Theta_{x} \otimes Q^{-1} \otimes P \otimes \Theta_{y} \otimes P^{-1} \otimes \Theta_{y^{-1}} \quad \rightarrow \quad P \otimes \Theta_{y} \otimes P^{-1} \otimes \Theta_{y^{-1}} \otimes \Theta_{x^{-1}} \otimes Q \otimes \Theta_{x} \otimes Q^{-1}$ $u_{x^{-1}} \otimes q \otimes u_{x} \otimes \bar{q} \otimes p \otimes u_{y} \otimes \bar{p} \otimes u_{y^{-1}} \quad \mapsto \sum f_{i}^{x}\left(u_{x^{-1}} \otimes q \otimes u_{x} \otimes \bar{q}\right) p \otimes u_{y} \otimes \bar{p} \otimes u_{y^{-1}} \otimes g_{i}^{x}(1)$.

Para construir $T_{2}$, vamos considerar as aplicações $R$-bilineares $f_{i, j}^{x y}: 1_{y^{-1}} \Theta_{x y} \otimes Q \otimes 1_{x} \Theta_{x y} \otimes Q^{-1} \longrightarrow R$ 
e $g_{i, j}^{x y}: R \longrightarrow 1_{y^{-1}} \Theta_{x y} \otimes Q \otimes 1_{x} \Theta_{x y} \otimes Q^{-1}$ definidas da seguinte forma:

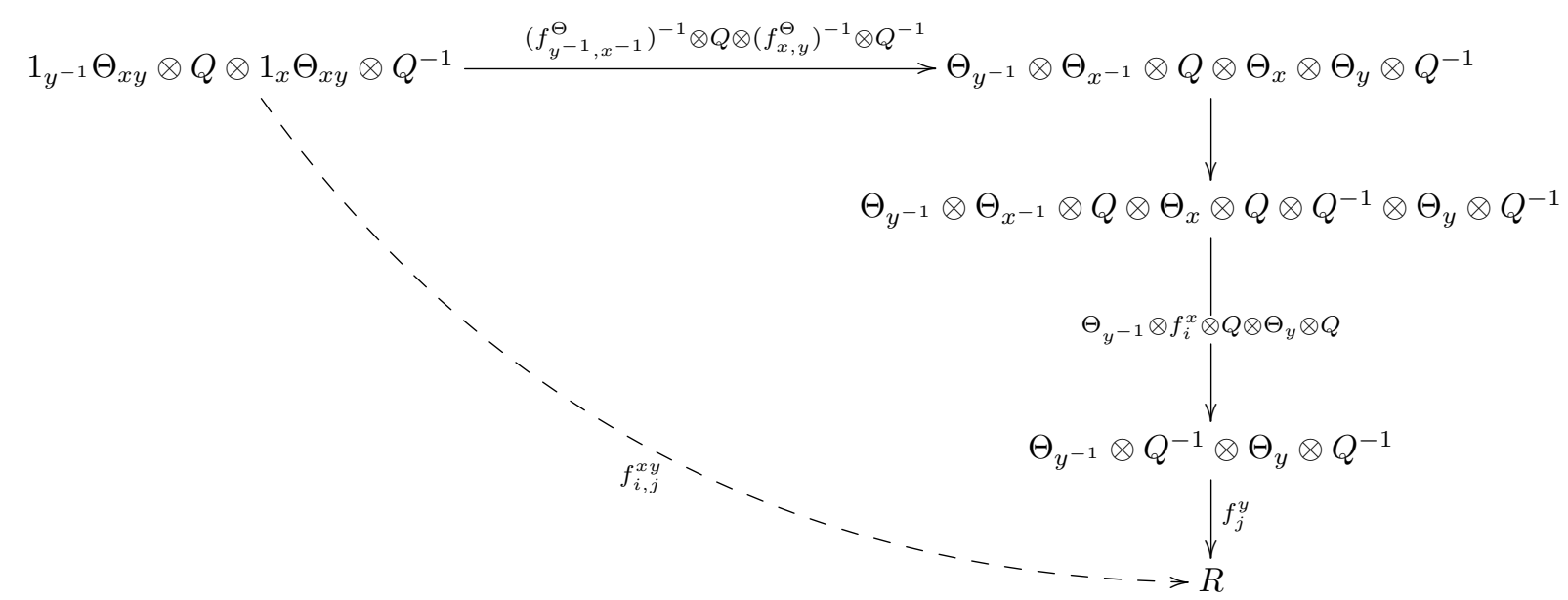

Então,

$$
f_{i, j}^{x y}\left(u_{(x y)^{-1}} \otimes q \otimes u_{x y} \otimes \bar{q}\right)=\sum f_{j}^{y}\left(u_{y^{-1}} f_{i}^{x}\left(u_{x^{-1}} \otimes q \otimes u_{x} \otimes \bar{q}_{e}\right) \otimes q_{e} \otimes u_{y} \otimes \bar{q}\right),
$$

onde $\sum\left(u_{y^{-1}} \stackrel{\ominus}{\circ} u_{x^{-1}}\right)=u_{(x y)^{-1}}, \sum\left(u_{x} \stackrel{\ominus}{\circ} u_{y}\right)=u_{x y}$ e $\sum_{t} \mathfrak{r}^{\prime}\left(\bar{q}_{t} \otimes q_{t}\right)=1$.

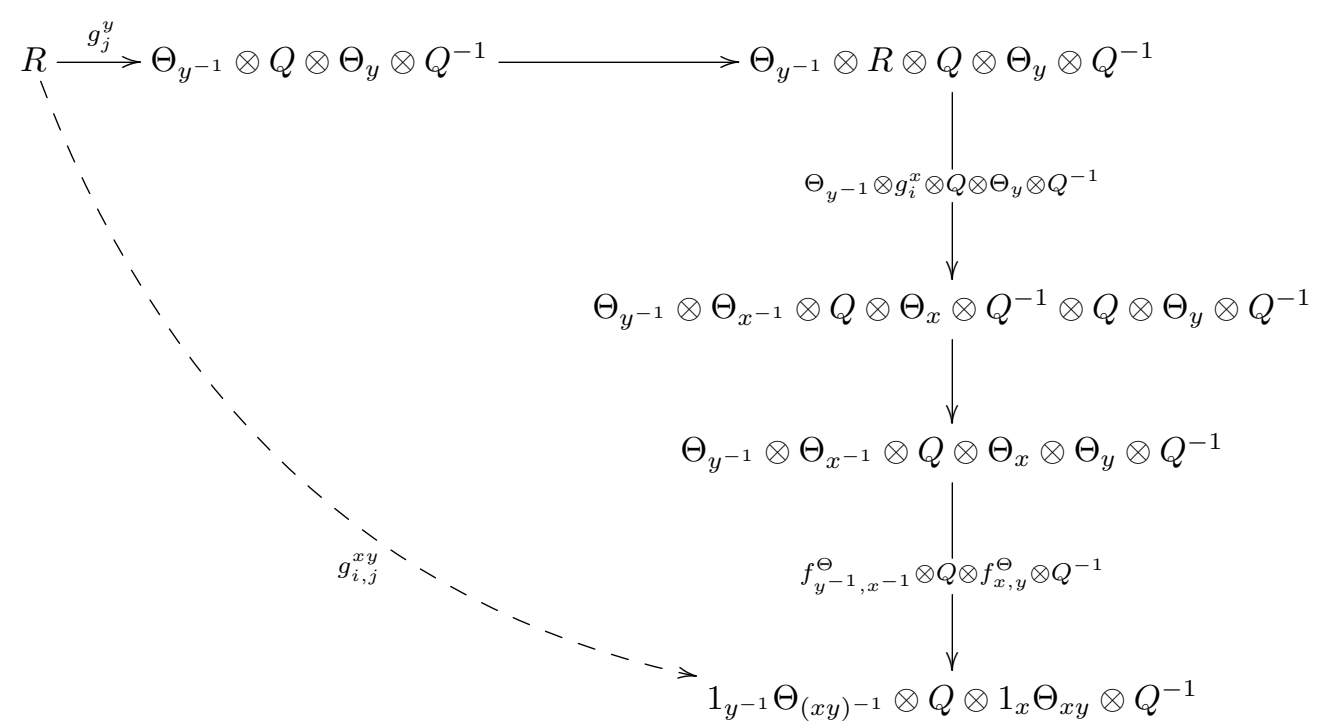

Então,

$$
g_{i, j}^{x y}(1)=\sum\left(\widetilde{u}_{y^{-1}}^{j, k} \stackrel{\ominus}{\circ} \widetilde{u}_{x^{-1}}^{i, l}\right) \otimes \widetilde{q}_{x}^{i, l} \otimes\left(\widetilde{u}_{x}^{i, l} \mathfrak{r}^{\prime}\left(\widetilde{q}_{x^{-1}}^{i, l} \otimes \widetilde{q}_{y}^{j, k}\right) \stackrel{\ominus}{\circ} \widetilde{u}_{y}^{j, k}\right) \otimes \widetilde{q}_{y^{-1}}^{j, k} .
$$

É fácil ver que $\sum_{i, j} g_{i, j}^{x y} f_{i, j}^{x y}=I d_{1_{y^{-1}} \Theta_{(x y)^{-1}} \otimes Q \otimes 1_{x} \Theta_{x y} \otimes Q^{-1}}$. Então, o isomorfismo $T_{2}$ é dado por

$$
\begin{aligned}
T_{2}: \quad P^{-1} \otimes \Theta_{(x y)^{-1}} \otimes Q \otimes \Theta_{x y} \otimes Q^{-1} & \longrightarrow \Theta_{(x y)^{-1}} \otimes Q \otimes \Theta_{x y} \otimes Q^{-1} \otimes P^{-1} \\
\bar{p} \otimes u_{(x y)^{-1}} \otimes q \otimes u_{x y} \otimes \bar{q} & \longmapsto \sum g_{i, j}^{x y}(1) \otimes \bar{p} f_{i, j}^{x y}\left(u_{(x y)^{-1}} \otimes q \otimes u_{x y} \otimes \bar{q}\right) .
\end{aligned}
$$

Pelo Exemplo 2.3.7, temos que os isomorfismos $T_{3}, T_{6}$ e $T_{7}$ são definidos por:

$$
\begin{aligned}
& R 1_{x y} \otimes Q \stackrel{T_{3}}{\longrightarrow} Q \otimes R 1_{x y}, \quad R 1_{x} \otimes Q \quad \stackrel{T_{6}}{\longrightarrow} Q \otimes R 1_{x}, \quad R 1_{y} \otimes Q \quad \stackrel{T_{7}}{\longrightarrow} \quad Q \otimes R 1_{y} \\
& r \otimes q \longmapsto r q \otimes 1_{x y}, \quad r \otimes q \longmapsto r q \otimes 1_{x} \quad, \quad r \otimes q \longmapsto r q \otimes 1_{y} \text {. }
\end{aligned}
$$


Vamos calcular primeiro $f_{x, y}^{P \otimes Q} \circ\left(F_{x} \otimes F_{y}\right)$ :

$p_{1} \otimes u_{x} \otimes \bar{p}_{1} \otimes u_{x^{-1}} \otimes q_{1} \otimes u_{x}^{\prime} \otimes \bar{q}_{1} \otimes p_{2} \otimes u_{y} \otimes \bar{p}_{2} \otimes u_{y^{-1}} \otimes q_{2} \otimes u_{y}^{\prime} \otimes \bar{q}_{2}$

$\stackrel{T_{4}, T_{5}}{\longmapsto} \sum_{i, l, j, k} p_{1} \otimes u_{x} \otimes u_{x^{-1}}^{i, l} \otimes q_{x}^{i, l} \otimes u_{x}^{i, l} \otimes q_{x-1}^{i, l} \otimes \bar{p}_{1} \underbrace{f_{i}^{x}\left(u_{x^{-1}} \otimes q_{1} \otimes u_{x}^{\prime} \otimes \bar{q}_{1}\right)}_{=a_{i}^{x} \in R} \otimes p_{2} \otimes u_{y}$

$\otimes \widetilde{u}_{y^{-1}}^{j, k} \otimes \widetilde{q}_{y}^{j, k} \otimes \widetilde{u}_{y}^{j, k} \otimes \widetilde{q}_{y^{-1}}^{j, k} \otimes \bar{p}_{2} \underbrace{f_{j}^{y}\left(u_{y^{-1}} \otimes q_{2} \otimes u_{y}^{\prime} \otimes \bar{q}_{2}\right)}_{=a_{j}^{y} \in R}$

$\mapsto \quad \sum_{i, l, j, k} p_{1} \otimes\left(u_{x} \stackrel{\ominus}{\circ} u_{x^{-1}}^{i, l}\right) \otimes q_{x}^{i, l} \otimes u_{x}^{i, l} \otimes q_{x^{-1}}^{i, l} \otimes \bar{p}_{1} a_{i}^{x} \otimes p_{2} \otimes\left(u_{y} \stackrel{\ominus}{\circ} \widetilde{u}_{y^{-1}}^{j, k}\right) \otimes \widetilde{q}_{y}^{j, k} \otimes \widetilde{u}_{y}^{j, k} \otimes \widetilde{q}_{y^{-1}}^{j, k} \otimes \bar{p}_{2} a_{j}^{y}$

$\stackrel{T_{6}, T_{7}}{\longmapsto} \sum_{i, l, j, k} p_{1} \otimes\left(u_{x} \stackrel{\ominus}{\circ} u_{x^{-1}}^{i, l}\right) q_{x}^{i, l} \otimes 1_{x} \otimes u_{x}^{i, l} \otimes q_{x^{-1}}^{i, l} \otimes \bar{p}_{1} a_{i}^{x} \otimes p_{2} \otimes\left(u_{y} \stackrel{\ominus}{\circ} \widetilde{u}_{y^{-1}}^{j, k}\right) \widetilde{q}_{y}^{j, k} \otimes 1_{y} \otimes \widetilde{u}_{y}^{j, k} \otimes \widetilde{q}_{y^{-1}}^{j, k} \otimes \bar{p}_{2} a_{j}^{y}$

$\mapsto \quad \sum_{i, l, j, k} p_{1} \otimes\left(u_{x} \stackrel{\ominus}{\circ} u_{x^{-1}}^{i, l}\right) q_{x}^{i, l} \otimes u_{x}^{i, l} \otimes q_{x^{-1}}^{i, l} \otimes \bar{p}_{1} a_{i}^{x} \otimes p_{2} \otimes\left(u_{y} \stackrel{\ominus}{\circ} \widetilde{u}_{y^{-1}}^{j, k}\right) \widetilde{q}_{y}^{j, k} \otimes \widetilde{u}_{y}^{j, k} \otimes \widetilde{q}_{y^{-1}}^{j, k} \otimes \bar{p}_{2} a_{j}^{y}$

$\mapsto \sum_{i, l, j, k} p_{1} \otimes\left(u_{x} \stackrel{\ominus}{\circ} u_{x^{-1}}^{i, l}\right) q_{x}^{i, l} \otimes(u_{x}^{i, l} \underbrace{\mathfrak{r}^{\prime}\left(\widetilde{q}_{x^{-1}}^{i, l} \mathfrak{r}\left(\bar{p}_{1} a_{i}^{x} \otimes p_{2}\right) \otimes\left(u_{y} \stackrel{\ominus}{\circ} \widetilde{u}_{y^{-1}}^{j, k}\right) \widetilde{q}_{y}^{j, k}\right)}_{=a \in R} \otimes \stackrel{\ominus}{\circ} \widetilde{u}_{y}^{j, k}) \otimes \widetilde{q}_{y^{-1}}^{j, k} \otimes \bar{p}_{2} a_{j}^{y}$

$=\sum_{i, l, j, k} p_{1} \otimes\left(u_{x} \stackrel{\ominus}{\circ} u_{x^{-1}}^{i, l}\right) q_{x}^{i, l} \otimes\left(u_{x}^{i, l} a \otimes \stackrel{\ominus}{\circ} \widetilde{u}_{y}^{j, k}\right) \otimes \widetilde{q}_{y^{-1}}^{j, k} \otimes \bar{p}_{2} f_{j}^{y}\left(u_{y^{-1}} \otimes q_{2} \otimes u_{y}^{\prime} \otimes \bar{q}_{2}\right)$,

Para $F_{x y} \circ f_{x, y}^{P Q}$, temos:

$p_{1} \otimes u_{x} \otimes \bar{p}_{1} \otimes u_{x^{-1}} \otimes q_{1} \otimes u_{x}^{\prime} \otimes \bar{q}_{1} \otimes p_{2} \otimes u_{y} \otimes \bar{p}_{2} \otimes u_{y^{-1}} \otimes q_{2} \otimes u_{y}^{\prime} \otimes \bar{q}_{2}$

$\stackrel{T_{7}}{\mapsto} \sum_{h, l^{\prime}} p_{1} \otimes u_{x} \otimes \bar{p}_{1} \otimes f_{h}^{x}\left(u_{x^{-1}} \otimes q_{1} \otimes u_{x}^{\prime} \otimes \bar{q}_{1}\right) p_{2} \otimes u_{y} \otimes \bar{p}_{2} \otimes u_{y^{-1}} \otimes u_{x^{-1}}^{h, l^{\prime}} \otimes q_{x}^{h, l^{\prime}}$ $\otimes u_{x}^{h, l^{\prime}} \otimes q_{x^{-1}}^{h, l^{\prime}} \otimes q_{2} \otimes u_{y}^{\prime} \otimes \bar{q}_{2}$

$\mapsto \sum_{h, l^{\prime}} p_{1} \otimes\left(u_{x} \mathfrak{r}\left(\bar{p}_{1} f_{h}^{x}\left(u_{x^{-1}} \otimes q_{1} \otimes u_{x}^{\prime} \otimes \bar{q}_{1}\right) \otimes p_{2}\right) \stackrel{\ominus}{\circ} u_{y}\right) \otimes \bar{p}_{2} \otimes\left(u_{y^{-1}} \stackrel{\ominus}{\circ} u_{x^{-1}}^{h, l^{\prime}}\right) \otimes q_{x}^{h, l^{\prime}}$ $\otimes\left(u_{x}^{h, l^{\prime}} \mathfrak{r}^{\prime}\left(q_{x^{-1}}^{h, l^{\prime}} \otimes q_{2}\right) \stackrel{\ominus}{\circ} u_{y}^{\prime}\right) \otimes \bar{q}_{2}$

$\stackrel{T_{2}}{\longmapsto} \sum_{h, l^{\prime}, i, l, j, k, t} p_{1} \otimes\left(u_{x} \mathfrak{r}\left(\bar{p}_{1} f_{h}^{x}\left(u_{x^{-1}} \otimes q_{1} \otimes u_{x}^{\prime} \otimes \bar{q}_{1}\right) \otimes p_{2}\right) \stackrel{\ominus}{\circ} u_{y}\right) \otimes\left(\widetilde{u}_{y^{-1}}^{j, k} \stackrel{\ominus}{\circ} u_{x^{-1}}^{h, l^{\prime}}\right) \otimes q_{x}^{h, l^{\prime}}$ $\otimes\left(u_{x}^{h, l^{\prime}} \mathfrak{r}^{\prime}\left(q_{x^{-1}}^{h, l^{\prime}} \otimes \widetilde{q}_{y}^{j, k}\right) \stackrel{\Theta}{\circ} \widetilde{u}_{y}^{j, k}\right) \otimes \widetilde{q}_{y^{-1}}^{j, k} \otimes \bar{p}_{2} f_{j}^{y}\left(u_{y^{-1}} f_{i}^{x}\left(u_{x^{-1}}^{h, l^{\prime}} \otimes q_{x}^{h, l^{\prime}} \otimes u_{x}^{h, l^{\prime}} \mathfrak{r}^{\prime}\left(q_{x^{-1}}^{h, l^{\prime}} \otimes q_{2}\right) \otimes \bar{q}_{t}\right)\right.$ $\left.\otimes q_{t} \otimes u_{y}^{\prime} \otimes \bar{q}_{2}\right)$

$=\sum_{h, l^{\prime}, i, l, j, k, t} p_{1} \otimes\left(u_{x} \mathfrak{r}\left(\bar{p}_{1} f_{h}^{x}\left(u_{x^{-1}} \otimes q_{1} \otimes u_{x}^{\prime} \otimes \bar{q}_{1}\right) \otimes p_{2}\right) \stackrel{\ominus}{\circ} u_{y}\right) \otimes\left(\widetilde{u}_{y^{-1}}^{j, k} \stackrel{\ominus}{\circ} u_{x^{-1}}^{i, l}\right) \otimes q_{x}^{i, l}$ $\otimes\left(u_{x}^{i, l} \mathfrak{r}^{\prime}\left(q_{x^{-1}}^{i, l} \otimes \widetilde{q}_{y}^{j, k}\right) \stackrel{\Theta}{\circ} \widetilde{u}_{y}^{j, k}\right) \otimes \widetilde{q}_{y^{-1}}^{j, k} \otimes \bar{p}_{2} f_{j}^{y}\left(u_{y^{-1}} f_{i}^{x}\left(u_{x^{-1}}^{h, l^{\prime}} \otimes q_{x}^{h, l^{\prime}} \otimes u_{x}^{h, l^{\prime}} \otimes q_{x^{-1}}^{h, l^{\prime}} \mathfrak{l}^{\prime}\left(q_{2} \otimes \bar{q}_{t}\right)\right)\right.$ $\left.\otimes q_{t} \otimes u_{y}^{\prime} \otimes \bar{q}_{2}\right)$ 


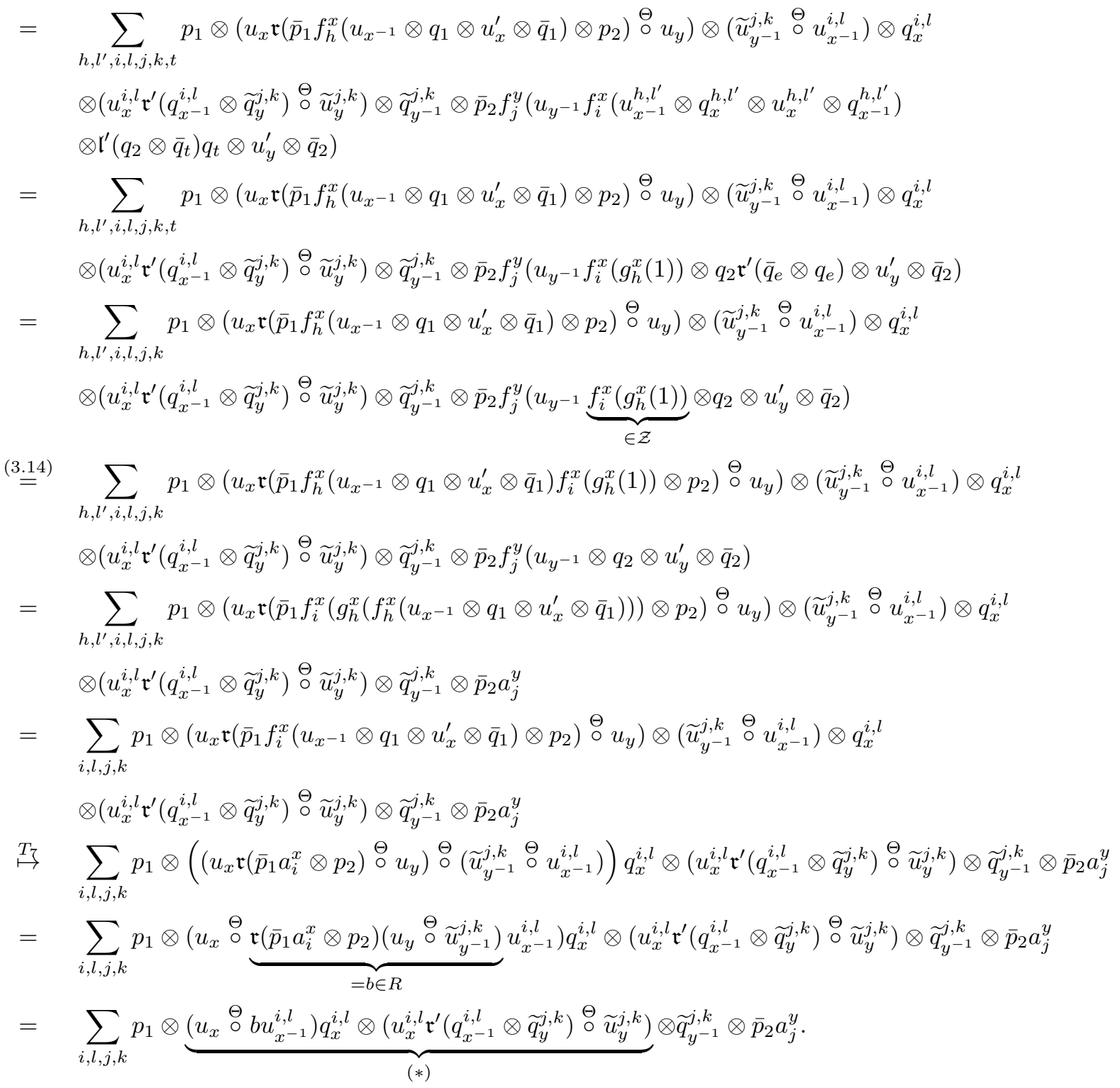

Observe que $(*)$ pode ser escrito como:

$$
\begin{aligned}
& \left(u_{x} \stackrel{\ominus}{\circ} b u_{x^{-1}}^{i, l}\right) q_{x}^{i, l} \otimes\left(u_{x}^{i, l} \mathfrak{r}^{\prime}\left(q_{x^{-1}}^{i, l} \otimes \widetilde{q}_{y}^{j, k}\right) \stackrel{\ominus}{\circ} \widetilde{u}_{y}^{j, k}\right) \\
= & \left(Q \otimes f_{x, y}^{\Theta}\right) \circ\left(f_{x, x^{-1}}^{\Theta} \otimes Q \otimes \Theta_{x} \mathfrak{r}^{\prime} \otimes \Theta_{y}\right)(u_{x} \otimes b \underbrace{u_{x^{-1}}^{i, l} \otimes q_{x}^{i, l} \otimes u_{x}^{i, l} \otimes q_{x-1}^{i, l}}_{\in C_{\Theta_{x^{-1}} \otimes Q \otimes \Theta_{x} \otimes Q^{-1}}(R)} \otimes \widetilde{q}_{y}^{j, k} \otimes \widetilde{u}_{y}^{j, k}) \\
= & \left(Q \otimes f_{x, y}^{\Theta}\right) \circ\left(f_{x, x^{-1}}^{\Theta} \otimes Q \otimes \Theta_{x} \mathfrak{r}^{\prime} \otimes \Theta_{y}\right)\left(u_{x} \otimes u_{x^{-1}}^{i, l} \otimes q_{x}^{i, l} \otimes u_{x}^{i, l} \otimes q_{x^{-1}}^{i, l} b \otimes \widetilde{q}_{y}^{j, k} \otimes \widetilde{u}_{y}^{j, k}\right) \\
= & \left(u_{x} \stackrel{\ominus}{\circ} u_{x^{-1}}^{i, l}\right) q_{x}^{i, l} \otimes\left(u_{x}^{i, l} \mathfrak{r}^{\prime}\left(q_{x^{-1}}^{i, l} \mathfrak{r}\left(\bar{p}_{1} a_{i}^{x} \otimes p_{2}\right)\left(u_{y} \stackrel{\ominus}{\circ} \widetilde{u}_{y^{-1}}^{j, k}\right) \otimes \widetilde{q}_{y}^{j, k}\right) \stackrel{\ominus}{\circ} \widetilde{u}_{y}^{j, k}\right) \\
= & \left(u_{x} \stackrel{\ominus}{\circ} u_{x^{-1}}^{i, l}\right) q_{x}^{i, l} \otimes\left(u_{x}^{i, l} \mathfrak{r}^{\prime}\left(q_{x^{-1}}^{i, l} \mathfrak{r}\left(\bar{p}_{1} a_{i}^{x} \otimes p_{2}\right) \otimes\left(u_{y} \stackrel{\ominus}{\circ} \widetilde{u}_{y^{-1}}^{j, k}\right) \widetilde{q}_{y}^{j, k}\right) \stackrel{\ominus}{\circ} \widetilde{u}_{y}^{j, k}\right) \\
= & \left(u_{x} \stackrel{\ominus}{\circ} u_{x^{-1}}^{i, l}\right) q_{x}^{i, l} \otimes\left(u_{x}^{i, l} a \stackrel{\ominus}{\circ} \widetilde{u}_{y}^{j, k}\right)
\end{aligned}
$$


Logo, temos que

$$
\begin{aligned}
& \left(F_{x y} \circ f_{x, y}^{P Q}\right)\left(p_{1} \otimes u_{x} \otimes \bar{p}_{1} \otimes u_{x^{-1}} \otimes q_{1} \otimes u_{x}^{\prime} \otimes \bar{q}_{1} \otimes p_{2} \otimes u_{y} \otimes \bar{p}_{2} \otimes u_{y^{-1}} \otimes q_{2} \otimes u_{y}^{\prime} \otimes \bar{q}_{2}\right) \\
= & \sum_{i, l, j, k} p_{1} \otimes\left(u_{x} \stackrel{\ominus}{\circ} u_{x^{-1}}^{i, l}\right) q_{x}^{i, l} \otimes\left(u_{x}^{i, l} a \stackrel{\ominus}{\circ} \widetilde{u}_{y}^{j, k}\right) \otimes \widetilde{q}_{y^{-1}}^{j, k} \otimes \bar{p}_{2} f_{j}^{y}\left(u_{y^{-1}} \otimes q_{2} \otimes u_{y}^{\prime} \otimes \bar{q}_{2}\right)
\end{aligned}
$$

Portanto $\mathcal{L}$ é um morfismo de grupos.

Por (4.3), temos que se $[P] \in \mathbf{P i c}_{\mathcal{Z}}(R) \cap \mathbf{P i c S}_{\mathcal{Z}}(R)^{\alpha^{*}}$, então $\left[\Delta\left(\Omega^{P}\right)\right] \in \mathcal{C}_{0}(\Theta / R)$. Definimos o morfismo $\varphi_{3}: \mathbf{P i c}_{\mathcal{Z}}(R) \cap \mathbf{P i c S}_{\mathcal{Z}}(R)^{\alpha^{*}} \longrightarrow \mathcal{C}_{0}(\Theta / R)$ como sendo a restrição do morfismo $\mathcal{L}$. Ou seja,

$$
\begin{aligned}
\varphi_{3}: \mathbf{P i c}_{\mathcal{Z}}(R) \cap \mathbf{P i c S}_{\mathcal{Z}}(R)^{\alpha^{*}} & \longrightarrow \mathcal{C}_{0}(\Theta / R) \\
{[P] } & \longmapsto\left[\Delta\left(\Omega^{P}\right)\right]
\end{aligned}
$$

Teorema 4.2.7. A sequência

$$
\mathcal{P}_{\mathcal{Z}}(\Delta(\Theta) / R)^{(G)} \stackrel{\varphi_{2}}{\longrightarrow} \operatorname{Pic}_{\mathcal{Z}}(R) \cap \operatorname{PicS}_{\mathcal{Z}}(R)^{\alpha^{*}} \stackrel{\varphi_{3}}{\longrightarrow} \mathcal{C}_{0}(\Theta / R)
$$

é exata.

Demonstração. Seja $[Q] \in \operatorname{Im}\left(\varphi_{2}\right)$, então existe $[Q]=[\phi] \Longrightarrow[X] \in \mathcal{P}_{\mathcal{Z}}(\Delta(\Theta) / R)^{(G)}$. Pela Proposição 4.1.3 existe um isomorfismo de $R$-bimódulos $g_{x}: \Theta_{x} \otimes Q \otimes \Theta_{x^{-1}} \longrightarrow Q \otimes R 1_{x}$ definido por

$$
g_{x}\left(u_{x} \otimes q \otimes u_{x^{-1}}\right)=q^{\prime} \otimes 1_{x}, \text { onde } \phi\left(q^{\prime}\right)=u_{x} \phi(q) u_{x^{-1}}
$$

Seja $h_{x}: Q \otimes \Theta_{x} \longrightarrow \Theta_{x} \otimes Q$ o isomorfismo de $R$-bimódulos que faz o diagrama abaixo comutar

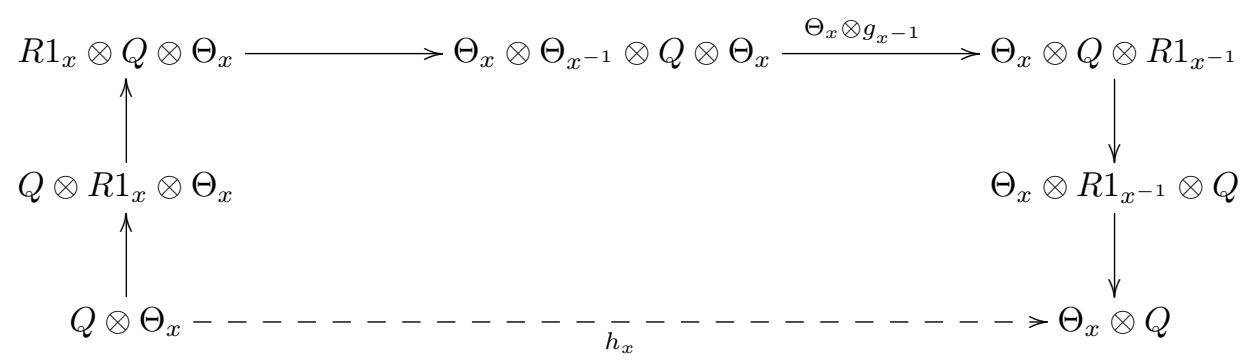

ou seja,

$$
h_{x}\left(q \otimes u_{x}\right)=\sum_{(x)} \omega_{x} \otimes g_{x^{-1}}\left(\omega_{x^{-1}} \otimes q \otimes u_{x}\right)
$$

onde $1_{x}=\sum_{(x)} \omega_{x} \stackrel{\ominus}{\circ} \omega_{x^{-1}}, \operatorname{com} \omega_{x} \in \Theta_{x}$ e $\omega_{x^{-1}} \in \Theta_{x^{-1}}$.

Afirmação 4.2.8. Os isomorfismos $h_{x}$ satisfazem o diagrama comutativo: 


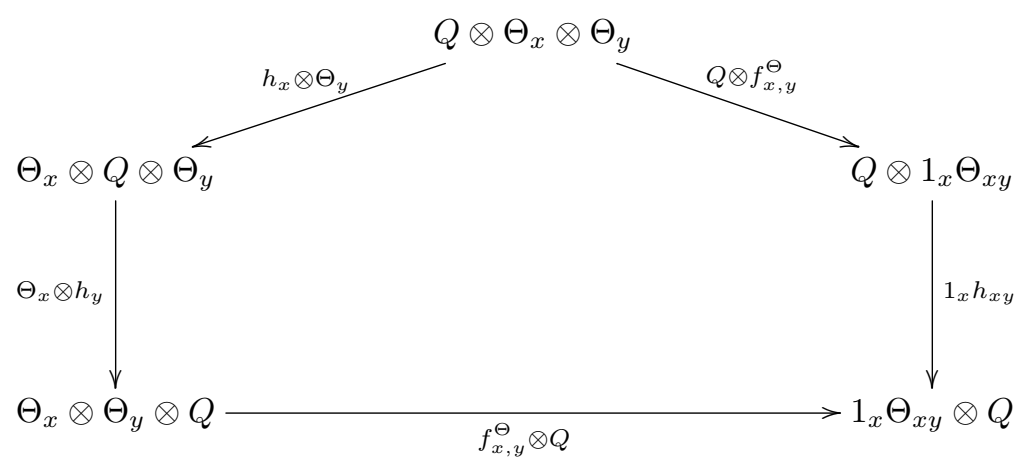

De fato, sejam $1_{x}=\sum_{(x)}\left(\omega_{x} \stackrel{\ominus}{\circ} \omega_{x^{-1}}\right)$ e $1_{y}=\sum_{(y)}\left(\omega_{y} \stackrel{\ominus}{\circ} \omega_{y^{-1}}\right)$. Pela Observação 3.3.10, a restrição do isomorfismo $1_{x} h_{x y}: Q \otimes 1_{x} \Theta_{x y} \longrightarrow 1_{x} \Theta_{x y} \otimes Q$ é dado por

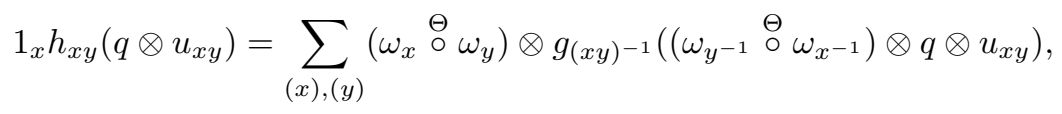

para todo $u_{x y} \in 1_{x} \Theta_{x y}$. Assim, dados $q \in Q, u_{x} \in \Theta_{x}$ e $u_{y} \in \Theta_{y}$, temos

$$
\begin{aligned}
1_{x} h_{x y} \circ\left(Q \otimes f_{x, y}^{\ominus}\right)\left(q \otimes u_{x} \otimes u_{y}\right) & =1_{x} h_{x y}\left(q \otimes\left(u_{x} \stackrel{\ominus}{\circ} u_{y}\right)\right) \\
& =\sum_{(x),(y)}\left(\omega_{x} \stackrel{\ominus}{\circ} \omega_{y}\right) \otimes g_{(x y)^{-1}}\left(\left(\omega_{y^{-1}} \stackrel{\ominus}{\circ} \omega_{x^{-1}}\right) \otimes q \otimes\left(u_{x} \stackrel{\ominus}{\circ} u_{y}\right)\right) \\
& =\sum_{(x),(y)}\left(\omega_{x} \stackrel{\ominus}{\circ} \omega_{y}\right) \otimes q_{x, y},
\end{aligned}
$$

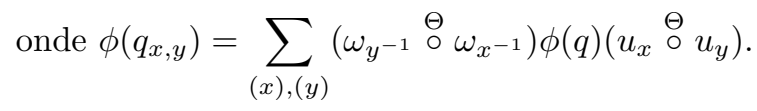

Por outro lado, aplicando $\left(f_{x, y}^{\Theta} \otimes Q\right) \circ\left(\Theta_{x} \otimes h_{y}\right) \otimes\left(h_{x} \otimes \Theta_{y}\right)$ temos:

$$
\begin{aligned}
& q \otimes u_{x} \otimes u_{y} \stackrel{h_{x} \otimes \Theta_{y}}{\mapsto} \sum_{(x)} \omega_{x} \otimes g_{x}\left(\omega_{x^{-1}} \otimes q \otimes u_{x}\right) \otimes u_{y} \\
& =\sum_{(x)} \omega_{x} \otimes q_{x}^{\prime} \otimes u_{y}, \text { onde } \phi\left(q_{x}^{\prime}\right)=\sum_{(x)} \omega_{x^{-1}} \phi(q) u_{x}, \\
& \stackrel{\Theta_{x} \otimes h_{y}}{\mapsto} \sum_{(x),(y)} \omega_{x} \otimes \omega_{y} \otimes g_{y}\left(\omega_{y^{-1}} \otimes q_{x} \otimes u_{y}\right) \\
& =\sum_{(x),(y)} \omega_{x} \otimes \omega_{y} \otimes q_{x, y}^{\prime} \\
& \stackrel{\ominus}{f_{x, y}^{\ominus} \otimes Q} \sum_{(x),(y)}\left(\omega_{x} \stackrel{\ominus}{\circ} \omega_{y}\right) \otimes q_{x, y}^{\prime},
\end{aligned}
$$

onde

$$
\begin{aligned}
\phi\left(q_{x, y}^{\prime}\right) & =\sum_{(x),(y)} \omega_{y^{-1}} \phi\left(q_{x}^{\prime}\right) u_{y}=\sum_{(x),(y)} \omega_{y^{-1}}\left(\omega_{x^{-1}} \phi(q) u_{x}\right) u_{y} \\
& =\sum_{(x),(y)}\left(\omega_{y^{-1}} \stackrel{\ominus}{\circ} \omega_{x^{-1}}\right) \phi(q)\left(u_{x} \stackrel{\ominus}{\circ} u_{y}\right)=\phi\left(q_{x, y}\right) .
\end{aligned}
$$


Como $\phi$ é injetora, temos que $q_{x, y}^{\prime}=q_{x, y}$. Logo, de (4.7) e (4.8) temos que o diagrama é comutativo.

A comutatividade do diagrama (4.6) pode ser expressa na seguinte igualdade: dados $q \in Q, u_{x} \in \Theta_{x}$ e $u_{y} \in \Theta_{y}$, denote

$$
h_{x}\left(q \otimes u_{x}\right)=\sum_{i=1}^{n} u_{x}^{i} \otimes q_{i} \quad \text { e } h_{y}\left(q_{i} \otimes u_{y}\right)=\sum_{j=1}^{m} u_{y}^{i, j} \otimes q_{i, j} .
$$

Então,

$$
h_{x y}\left(q \otimes\left(u_{x} \stackrel{\ominus}{\circ} u_{y}\right)\right)=\sum_{i, j}\left(u_{x}^{i} \stackrel{\ominus}{\circ} u_{y}^{i, j}\right) \otimes q_{i, j} .
$$

Considere agora o isomorfismo de $R$-bimódulos $F_{x}: Q \otimes \Theta_{x} \otimes Q^{-1} \longrightarrow \Theta_{x}$ definido por $F_{x}:=h_{x} \otimes Q^{-1}$, ou seja, dados $q \in Q, u_{x} \in \Theta_{x}$ e $\bar{q} \in Q^{-1}$, denotando $h_{x}\left(q \otimes u_{x}\right)=\sum_{i=1}^{n} u_{x}^{i} \otimes q_{i}$, então

$$
F_{x}\left(q \otimes u_{x} \otimes \bar{q}\right)=\sum_{i=1}^{n} u_{x}^{i} \mathfrak{l}\left(q_{i} \otimes \bar{q}\right) \in Q
$$

onde $Q \otimes_{R} Q^{-1} \stackrel{\mathfrak{l}}{\longrightarrow} R \stackrel{\mathfrak{r}}{\longleftarrow} Q^{-1} \otimes_{R} Q$ são isomorfismos de $R$-bimódulos.

Afirmação 4.2.9. $\varphi_{3}([Q])=[\Delta(\Theta)] e m \mathcal{C}_{0}(\Theta / R)$.

De fato, basta verificar que o seguinte diagrama é comutativo

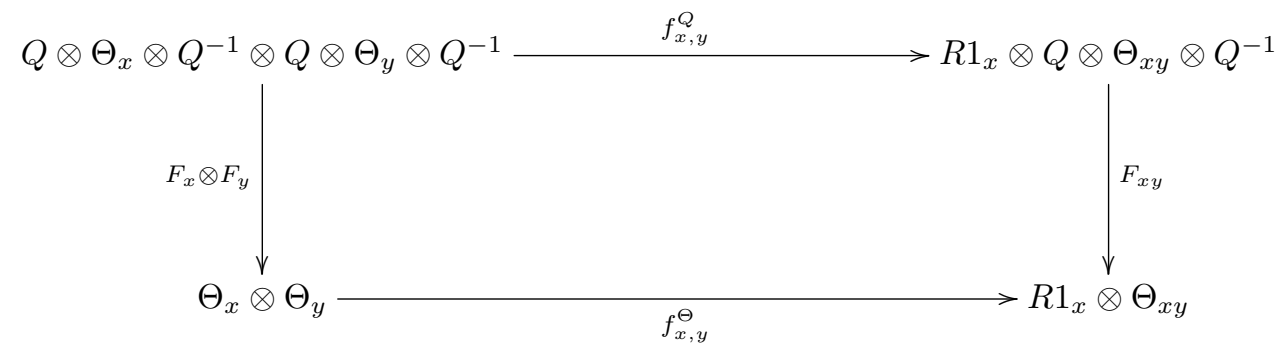

Sejam $q_{1}, q_{2} \in Q, \bar{q}_{1}, \bar{q}_{2} \in Q^{-1}, u_{x} \in \Theta_{x}$ e $u_{y} \in \Theta_{y}$. Denote

$$
h_{x}\left(q_{1} \otimes u_{x}\right)=\sum_{i=1}^{n} u_{x}^{i} \otimes q_{i} \quad \text { e } h_{y}\left(q_{i} \otimes \mathfrak{r}\left(\bar{q}_{1} \otimes q_{2}\right) u_{y}\right)=\sum_{j=1}^{m} u_{y}^{i, j} \otimes q_{i, j} .
$$

Então, por (4.9)

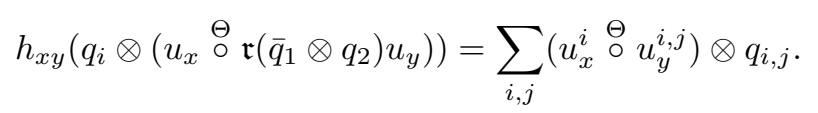

Assim,

$$
\begin{aligned}
\left(F_{x y} \circ f_{x, y}^{Q}\right)\left(q_{1} \otimes u_{x} \otimes \bar{q}_{1} \otimes q_{2} \otimes u_{y} \otimes \bar{q}_{2}\right) & =F_{x y}\left(q_{1} \otimes\left(u_{x} \stackrel{\ominus}{\circ} \mathfrak{r}\left(\bar{q}_{1} \otimes q_{2}\right) u_{y}\right) \otimes \bar{q}_{2}\right) \\
& =\sum_{i, j}\left(u_{x}^{i} \stackrel{\ominus}{\circ} u_{y}^{i, j}\right) \mathfrak{l}\left(q_{i, j} \otimes \bar{q}_{2}\right) .
\end{aligned}
$$


Por outro lado, aplicando $f_{x, y}^{\Theta} \circ\left(F_{x} \otimes F_{y}\right)$ em $q_{1} \otimes u_{x} \otimes \bar{q}_{1} \otimes q_{2} \otimes u_{y} \otimes \bar{q}_{2}$, temos

$$
\begin{aligned}
& \left(q_{1} \otimes u_{x} \otimes \bar{q}_{1} \otimes q_{2} \otimes u_{y} \otimes \bar{q}_{2}\right) \mapsto \sum_{i=1}^{n} u_{x}^{i} \mathfrak{l}\left(q_{i} \otimes \bar{q}_{1}\right) \otimes h_{y}\left(q_{2} \otimes u_{y}\right) \otimes \bar{q}_{2} \\
& =\sum_{i=1}^{n} u_{x}^{i} \otimes \mathfrak{l}\left(q_{i} \otimes \bar{q}_{1}\right) f_{y}\left(q_{2} \otimes u_{y}\right) \otimes \bar{q}_{2}=\sum_{i=1}^{n} u_{x}^{i} \otimes h_{y}\left(\mathfrak{l}\left(q_{i} \otimes \bar{q}_{1}\right) q_{2} \otimes u_{y}\right) \otimes \bar{q}_{2} \\
& =\sum_{i=1}^{n} u_{x}^{i} \otimes h_{y}\left(q_{i} \mathfrak{r}\left(\bar{q}_{1} \otimes q_{2}\right) \otimes u_{y}\right) \otimes \bar{q}_{2}=\sum_{i=1}^{n} u_{x}^{i} \otimes h_{y}\left(q_{i} \otimes \mathfrak{r}\left(\bar{q}_{1} \otimes q_{2}\right) u_{y}\right) \otimes \bar{q}_{2} \\
& =\sum_{i, j} u_{x}^{i} \otimes u_{y}^{i, j} \otimes q_{i, j} \otimes \bar{q}_{2}=\sum_{i, j} u_{x}^{i} \otimes u_{y}^{i, j} \mathfrak{l}\left(q_{i, j} \otimes \bar{q}_{2}\right) \\
& \mapsto \sum_{i, j}\left(u_{x}^{i} \stackrel{\ominus}{\circ} u_{y}^{i, j}\right) \mathfrak{l}\left(q_{i, j} \otimes \bar{q}_{2}\right)
\end{aligned}
$$

Logo, $F_{x} \circ f_{x, y}^{Q}=f_{x, y}^{\Theta} \circ\left(F_{x} \otimes F_{y}\right)$, para todo $x, y \in G$. Assim, $\Delta\left(\Omega^{Q}\right) \simeq \Delta(\Theta)$ como produtos cruzados generalizados parciais. Isso implica que $\varphi_{3}([Q])=\left[\Delta\left(\Omega^{Q}\right)\right]=[\Delta(\Theta)]$ em $\mathcal{C}_{0}(\Theta / R)$ e portanto $[Q] \in$ $\operatorname{ker}\left(\varphi_{3}\right)$.

Seja $[P] \in \operatorname{ker}\left(\varphi_{3}\right)$, então existe $j: \Delta\left(\Omega^{P}\right) \longrightarrow \Delta(\Theta)$ isomorfismo de produtos cruzados generalizados parciais. Ou seja, para cada $x \in G$, existe isomorfismo de $R$-bimódulos $j_{x}: P \otimes \Theta_{x} \otimes P^{-1} \longrightarrow \Theta_{x}$ tal que o diagrama

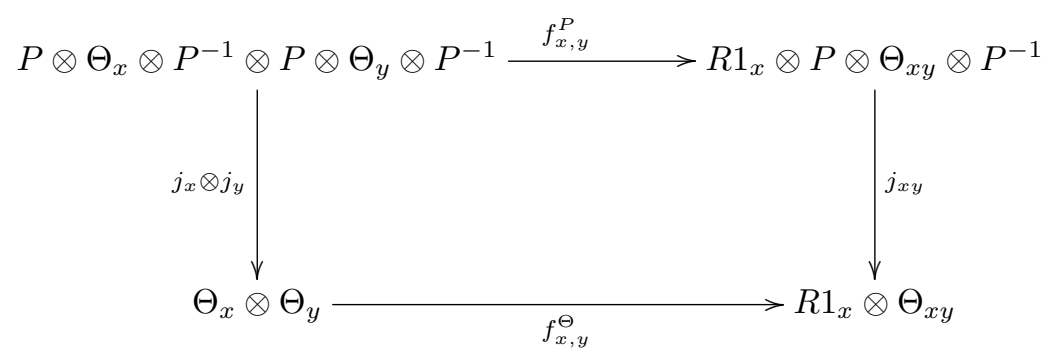

é comutativo. Dados $u_{x} \in \Theta_{x}$ e $u_{y} \in \Theta_{y}$, denote

$$
j_{x}^{-1}\left(u_{x}\right)=\sum_{i=1}^{n} p_{i} \otimes u_{x}^{i} \otimes \bar{p}_{i} \quad \text { e } j_{y}^{-1}\left(u_{y}\right)=\sum_{j=1}^{m} p_{j} \otimes u_{y}^{j} \otimes \bar{p}_{j} .
$$

Então, pela comutatividade do diagrama, temos

$$
j_{x y}^{-1}\left(u_{x} \stackrel{\ominus}{\circ} u_{y}\right)=\sum_{i, j} p_{i} \otimes\left(u_{x}^{i} \mathfrak{r}\left(\bar{p}_{i} \otimes p_{j}\right) \stackrel{\ominus}{\circ} u_{y}^{j}\right) \otimes \bar{p}_{j} .
$$

Seja $i_{x}: \Theta_{x} \otimes_{R} P \longrightarrow P \otimes_{R} \Theta_{x}$ definida por

$$
i_{x}: \Theta_{x} \otimes_{R} P \stackrel{j_{x}^{-1} \otimes P}{\longrightarrow} P \otimes \Theta_{x} \otimes P^{-1} \otimes P \longrightarrow P \otimes_{R} \Theta_{x}
$$

ou seja, dados $p \in P$ e $u_{x} \in \Theta_{x}$, se $j_{x}^{-1}\left(u_{x}\right)=\sum_{i=1}^{n} p_{i} \otimes u_{x}^{i} \otimes \bar{p}_{i}$, então

$$
i_{x}\left(u_{x} \otimes p\right)=\sum_{i=1}^{n} p_{i} \otimes u_{x}^{i} \mathfrak{r}\left(\bar{p}_{i} \otimes p\right) .
$$


Defina $i:=\bigoplus_{x \in G} i_{x}: \Delta(\Theta) \otimes_{R} P \longrightarrow P \otimes_{R} \Delta(\Theta)$. Vejamos que $i$ satisfaz as condições (2.13) e (2.14) do Lema 2.1.16. A primeira condição é:

$$
\left(P \otimes f_{x, y}^{\Theta}\right) \circ\left(i_{x} \otimes \Theta_{y}\right) \circ\left(\Theta_{x} \otimes i_{y}\right)=i_{x y} \circ\left(f_{x, y}^{\Theta} \otimes P\right) \quad \forall x, y \in G .
$$

Sejam $p \in P, u_{x} \in \Theta_{x}, u_{y} \in \Theta_{y}$ usando a notação em (4.11), então $i_{x y} \circ\left(f_{x, y}^{\Theta} \otimes P\right)$ é dado por:

$$
\begin{aligned}
u_{x} \otimes u_{y} \otimes p & \mapsto \quad\left(u_{x} \stackrel{\ominus}{\circ} u_{y}\right) \otimes p \mapsto j_{x y}^{-1}\left(u_{x} \stackrel{\ominus}{\circ} u_{y}\right) \otimes p \\
\stackrel{(4.12)}{=} & \sum_{i, j} p_{i} \otimes\left(u_{x}^{i} \mathfrak{r}\left(\bar{p}_{i} \otimes p_{j}\right) \stackrel{\ominus}{\circ} u_{y}^{j}\right) \otimes \bar{p}_{j} \otimes p \\
& \mapsto \sum_{i, j} p_{i} \otimes\left(u_{x}^{i} \mathfrak{r}\left(\bar{p}_{i} \otimes p_{j}\right) \stackrel{\ominus}{\circ} u_{y}^{j}\right) \mathfrak{r}\left(\bar{p}_{j} \otimes p\right) .
\end{aligned}
$$

Por outro lado, $\left(P \otimes f_{x, y}^{\Theta}\right) \circ\left(i_{x} \otimes \Theta_{y}\right) \circ\left(\Theta_{x} \otimes i_{y}\right)$ é dado por:

$$
\begin{aligned}
& u_{x} \otimes u_{y} \otimes p \stackrel{\Theta_{x} \otimes i_{y}}{\longmapsto} \sum_{j=1}^{m} u_{x} \otimes p_{j} \otimes u_{y}^{j} \mathfrak{r}\left(\bar{p}_{j} \otimes p\right) \\
& \stackrel{i_{x} \otimes \Theta_{y}}{\longmapsto} \sum_{i, j} p_{i} \otimes u_{x}^{i} \mathfrak{r}\left(\bar{p}_{i} \otimes p_{j}\right) \otimes u_{y}^{j} \mathfrak{r}\left(\bar{p}_{j} \otimes p\right) \\
& \stackrel{P \otimes f_{x, y}^{\Theta}}{\longmapsto} \sum_{i, j} p_{i} \otimes\left(u_{x}^{i} \mathfrak{r}\left(\bar{p}_{i} \otimes p_{j}\right) \stackrel{\ominus}{\circ} u_{y}^{j} \mathfrak{r}\left(\bar{p}_{j} \otimes p\right)\right) \\
&=\sum_{i, j} p_{i} \otimes\left(u_{x}^{i} \mathfrak{r}\left(\bar{p}_{i} \otimes p_{j}\right) \stackrel{\ominus}{\circ} u_{y}^{j}\right) \mathfrak{r}\left(\bar{p}_{j} \otimes p\right) .
\end{aligned}
$$

Logo, $i$ satisfaz a condição (2.13). Como $j$ é isomorfismo de produtos cruzados generalizados parciais, então $j_{1} \circ \nu_{P}=\iota$, onde $\nu_{P}$ é dado como na Observação 4.2.5. Então

$$
j_{1}^{-1}(1)=j_{1}^{-1}(\iota(1))=\nu_{P}(1)=\sum_{k=1}^{n} p_{k} \otimes \iota(1) \otimes \bar{p}_{k},
$$

onde $\sum_{k=1}^{n} \mathfrak{l}\left(p_{k} \otimes \bar{p}_{k}\right)=1$, para todo $r \in R$. Logo,

$$
\begin{aligned}
i_{1}(1 \otimes p) & =\sum_{k=1}^{n} p_{k} \otimes \mathfrak{r}\left(\bar{p}_{k} \otimes p\right)=\sum_{k=1}^{n} p_{k} \mathfrak{r}\left(\bar{p}_{k} \otimes p\right) \otimes 1 \\
& =\sum_{k=1}^{n} \mathfrak{r}\left(p_{k} \otimes \bar{p}_{k}\right) p \otimes 1=p \otimes 1 .
\end{aligned}
$$

Portanto, $i$ também satisfaz a condição (2.14). Segue do Lema 2.1.16 que $P \otimes_{R} \Delta(\Theta)$ é um $\Delta(\Theta)$-bimódulo via:

$$
\begin{gathered}
\left(p \otimes u_{x}\right) * u_{y}=p \otimes\left(u_{x} \stackrel{\ominus}{\circ} u_{y}\right) \\
u_{y} *\left(p \otimes u_{x}\right)=\sum_{i=1}^{n} p_{i} \otimes\left(u_{y}^{i} \stackrel{\ominus}{\circ} u_{x}\right), \text { onde } i_{y}\left(u_{y} \otimes p\right)=\sum_{i=1}^{n} p_{i} \otimes u_{y}^{i} .
\end{gathered}
$$

Vamos construir o isomorfismo $\overline{i_{x}}: \Theta_{x} \otimes_{R} P^{-1} \longrightarrow P^{-1} \otimes_{R} \Theta_{x}$ por:

$$
\overline{i_{x}}: \Theta_{x} \otimes_{R} P^{-1} \longrightarrow P^{-1} \otimes_{R} P \otimes_{R} \Theta_{x} \otimes_{R} P^{-1} \stackrel{j_{x}}{\longrightarrow} P^{-1} \otimes_{R} \Theta_{x},
$$


ou seja,

$$
\overline{i_{x}}\left(u_{x} \otimes \bar{p}\right)=\sum_{k=1}^{n} \bar{p}_{k} \otimes j_{x}\left(p_{k} \otimes u_{x} \otimes \bar{p}\right)
$$

onde $\sum_{k=1}^{n} \mathfrak{r}\left(\bar{p}_{x} \otimes p_{k}\right)=1$. Considere $\bar{i}=\bigoplus_{x \in G} \overline{i_{x}}: \Delta(\Theta) \otimes_{R} P \longrightarrow P \otimes_{R} \Delta(\Theta)$. Vamos verificar que $\bar{i}$ também satisfaz as condições do Lema 2.1.16. Sejam $u_{x} \in \Theta_{x}, u_{y} \in \Theta_{y}$ e $\bar{p} \in P^{-1}$. Considere $\sum_{k=1}^{n} \mathfrak{r}\left(\bar{p}_{k} \otimes p_{k}\right)=\sum_{l=1}^{m} \mathfrak{r}\left(\bar{p}_{l} \otimes p_{l}\right)=1$. Então, $\left(P \otimes f_{x, y}^{\Theta}\right) \circ\left(\overline{i_{x}} \otimes \Theta_{y}\right) \circ\left(\Theta_{x} \otimes \overline{i_{y}}\right)$ é dado por:

$$
\begin{aligned}
u_{x} \otimes u_{y} \otimes \bar{p} & \stackrel{\Theta_{x} \otimes \overline{i_{y}}}{\longmapsto} \sum_{l=1}^{m} u_{x} \otimes \bar{p}_{l} \otimes j_{y}\left(p_{l} \otimes u_{y} \otimes \bar{p}\right) \\
\stackrel{\bar{i}_{x} \otimes \Theta_{y}}{\longmapsto} & \sum_{k, l} \bar{p}_{k} \otimes j_{x}\left(p_{k} \otimes u_{x} \otimes \bar{p}_{l}\right) \otimes j_{y}\left(p_{l} \otimes u_{y} \otimes \bar{p}\right) \\
& \stackrel{P \otimes f_{x, y}^{\ominus}}{\longmapsto} \sum_{k, l} \bar{p}_{k} \otimes\left(j_{x}\left(p_{k} \otimes u_{x} \otimes \bar{p}_{l}\right) \stackrel{\ominus}{\circ} j_{y}\left(p_{l} \otimes u_{y} \otimes \bar{p}\right)\right) \\
& \stackrel{(*)}{=} \sum_{k, l} \bar{p}_{k} \otimes j_{x y}\left(p_{k} \otimes\left(u_{x} \mathfrak{r}\left(\bar{p}_{l} \otimes p_{l}\right) \stackrel{\ominus}{\circ} u_{y}\right) \otimes \bar{p}\right) \\
& =\sum_{k=1}^{n} \bar{p}_{k} \otimes j_{x y}\left(p_{k} \otimes\left(u_{x} \stackrel{\ominus}{\circ} u_{y}\right) \otimes \bar{p}\right) \\
& =i_{x y}\left(\left(u_{x} \stackrel{\ominus}{\circ} u_{y}\right) \otimes \bar{p}\right) \\
& =\quad i_{x y} \circ\left(f_{x, y}^{\Theta} \otimes P^{-1}\right)\left(u_{x} \otimes u_{y} \otimes \bar{p}\right),
\end{aligned}
$$

onde a igualdade $(*)$ vem da comutatividade do diagrama (4.10). Logo, temos $\left(P \otimes f_{x, y}^{\Theta}\right) \circ\left(\overline{i_{x}} \otimes \Theta_{y}\right) \circ$ $\left(\Theta_{x} \otimes \overline{i_{y}}\right)=\overline{i_{x y}} \circ\left(f_{x, y}^{\Theta} \otimes P^{-1}\right)$. Para a segunda condição temos

$$
\begin{aligned}
\overline{i_{1}}(1 \otimes \bar{p}) & =\sum_{k=1}^{n} \bar{p}_{k} \otimes j_{1}\left(p_{k} \otimes 1 \otimes \bar{p}\right)=\sum_{k=1}^{n} \bar{p}_{k} \otimes \mathfrak{l}\left(p_{k} \otimes \bar{p}\right) \\
& =\sum_{k=1}^{n} \bar{p}_{k} \mathfrak{l}\left(p_{k} \otimes \bar{p}\right) \otimes 1=\sum_{k=1}^{n} \mathfrak{r}\left(\bar{p}_{k} \otimes p_{k}\right) \bar{p} \otimes 1 \\
& =\bar{p} \otimes 1 .
\end{aligned}
$$

Segue do Lema 2.1.16 que $P^{-1} \otimes_{R} \Delta(\Theta)$ tem uma estrutura de $\Delta(\Theta)$-bimódulo dada por $\bar{i}$.

Afirmação 4.2.10. O isomorfismo $\bar{i}$ é dado por $\bar{i}=P^{-1} \otimes i^{-1} \otimes P^{-1}$, onde $P^{-1} \otimes i^{-1} \otimes P^{-1}: \Delta(\Theta) \otimes$ $P^{-1} \longrightarrow P^{-1} \otimes \Delta(\Theta)$ é definida como no item (i) Lema 2.1.19.

De fato, primeiramente observe que $i_{x}^{-1}: P \otimes \Theta_{x} \longrightarrow \Theta_{x} \otimes P$ é dado por

$$
i_{x}^{-1}\left(p \otimes u_{x}\right)=\sum_{l=1}^{m} j_{x}\left(p \otimes u_{x} \otimes \bar{p}_{l}\right) \otimes p_{l},
$$

onde $\sum_{l=1}^{m} \mathfrak{r}\left(\bar{p}_{l} \otimes p_{l}\right)=1$. Agora, aplicando $P^{-1} \otimes i^{-1} \otimes P^{-1}$ para $u_{x} \otimes \bar{p}$ temos: 


$$
\begin{aligned}
u_{x} \otimes \bar{p} & \mapsto \sum_{k=1}^{n} \bar{p}_{k} \otimes p_{k} \otimes u_{x} \otimes \bar{p} \mapsto \sum_{k=1}^{n} \bar{p}_{k} \otimes i^{-1}\left(p_{k} \otimes u_{x}\right) \otimes \bar{p} \\
& =\sum_{k, l} \bar{p}_{k} \otimes j_{x}\left(p_{k} \otimes u_{x} \otimes \bar{p}_{l}\right) \otimes p_{l} \otimes \bar{p} \\
& \mapsto \sum_{k, l} \bar{p}_{k} \otimes j_{x}\left(p_{k} \otimes u_{x} \otimes \bar{p}_{l}\right) \mathfrak{l}\left(p_{l} \otimes \bar{p}\right) \\
& =\sum_{k, l} \bar{p}_{k} \otimes j_{x}\left(p_{k} \otimes u_{x} \otimes \bar{p}_{l} \mathfrak{l}\left(p_{l} \otimes \bar{p}\right)\right) \\
& =\sum_{k, l} \bar{p}_{k} \otimes j_{x}\left(p_{k} \otimes u_{x} \otimes \mathfrak{r}\left(\bar{p}_{l} \otimes p_{l}\right) \bar{p}\right) \\
& =\sum_{k} \bar{p}_{k} \otimes j_{x}\left(p_{k} \otimes u_{x} \otimes \bar{p}\right) \\
& =\bar{i}_{x}\left(u_{x} \otimes \bar{p}\right) .
\end{aligned}
$$

Pelo item (iv) do Lema 2.1.19, temos que $\left[P \otimes_{R} \Delta(\Theta)\right] \in \operatorname{Pic}(\Delta(\Theta))$.

Vejamos que $[P]=[\phi] \Longrightarrow\left[P \otimes_{R} \Delta(\Theta)\right] \in \mathcal{P}_{\mathcal{Z}}(\Delta(\Theta) / R)^{(G)}$, onde

$$
\begin{aligned}
\phi: \quad P & \longrightarrow P \otimes_{R} \Delta(\Theta) \\
p & \longmapsto p \otimes 1 .
\end{aligned}
$$

Observe que

$$
\begin{aligned}
\bar{\phi}_{r}: \quad P \otimes_{R} \Delta(\Theta) & \longrightarrow P \otimes_{R} \Delta(\Theta) \\
p \otimes u_{x} & \longrightarrow \phi(p) * u_{x}
\end{aligned}
$$

é a identidade, pois

$$
\phi(p) * u_{x}=(p \otimes 1) * u_{x}=p \otimes u_{x}=p \otimes u_{x} .
$$

Assim, $\bar{\phi}_{r}$ é um isomorfismo de $R-\Delta(\Theta)$-bimódulos. Logo, $[P]=[\phi] \Longrightarrow\left[P \otimes_{R} \Delta(\Theta)\right] \in \mathcal{P}_{\mathcal{Z}}(\Delta(\Theta) / R)$. Vejamos que $\phi(P) * \Theta_{x}=\Theta_{x} * \phi(P)$, para todo $x \in G$. Sejam $p \in P$ e $u_{x} \in \Theta_{x}$. Denotando $i_{x}\left(u_{x} \otimes p\right)=$ $\sum_{i=1}^{n} p_{i} \otimes u_{x}^{i}$, temos

$$
\begin{aligned}
u_{x} * \phi(p) & =u_{x} *(p \otimes 1)=\sum_{i=1}^{n} p_{i} \otimes\left(u_{x}^{i} \stackrel{\ominus}{\circ} 1\right) \\
& =\sum_{i=1}^{n} p_{i} \otimes u_{x}^{i}=\sum_{i=1}^{n}\left(p_{i} \otimes 1\right) * u_{x}^{i} \\
& =\sum_{i=1}^{n} \phi\left(p_{i}\right) * u_{x}^{i} \in \phi(P) * \Theta_{x} .
\end{aligned}
$$

Logo, $\Theta_{x} * \phi(P) \subseteq \phi(P) * \Theta_{x}$. Por outro lado, como $p \otimes u_{x} \in P \otimes_{R} \Delta(\Theta)$ e $i: \Delta(\Theta) \otimes_{R} P \longrightarrow P \otimes_{R} \Delta(\Theta)$ é um isomorfismo, existem $u_{x}^{j} \in \Theta_{x}$ e $p_{j} \in P, \operatorname{com} j=1,2, \ldots, m$, tais que

$$
i_{x}\left(\sum_{j=1}^{m} u_{x}^{j} \otimes p_{j}\right)=p \otimes u_{x} .
$$


Então,

$$
\begin{aligned}
\phi(p) * u_{x} & =p \otimes u_{x}=i_{x}\left(\sum_{j=1}^{m} u_{x}^{j} \otimes p_{j}\right) \\
& =\sum_{j=1}^{m} u_{x}^{j} *\left(p_{j} \otimes 1\right)=\sum_{j=1}^{m} u_{x}^{j} * \phi\left(p_{j}\right) \in \Theta_{x} * \phi(P) .
\end{aligned}
$$

Assim, $\phi(P) \Theta_{x} \subseteq \Theta_{x} \phi(P)$ e consequentemente temos a igualdade. Portanto,

$$
[P]=[\phi] \Longrightarrow\left[P \otimes_{R} \Delta(\Theta)\right] \in \mathcal{P}_{\mathcal{Z}}(\Delta(\Theta) / R)^{(G)} .
$$

Claramente, $\varphi_{2}\left([P]=[\phi] \Longrightarrow\left[P \otimes_{R} \Delta(\Theta)\right]\right)=[P]$. Portanto, $[P] \in \operatorname{Im}\left(\varphi_{2}\right)$.

\subsection{O grupo $\mathcal{B}(\Theta / R)$ e a terceira sequência exata}

Definimos o grupo $\mathcal{B}(\Theta / R)$ via a sequência exata

$$
\operatorname{Pic}_{\mathcal{Z}}(R)^{(G)} \stackrel{\mathcal{L}}{\longrightarrow} \mathcal{C}(\Theta / R) \longrightarrow \mathcal{B}(\Theta / R) \longrightarrow 1
$$

ou seja,

$$
\mathcal{B}(\Theta / R)=\frac{\mathcal{C}(\Theta / R)}{\operatorname{Im}(\mathcal{L})}
$$

Notação: Seja $f: G_{1} \longrightarrow G_{2}$ um morfismo de grupos, vamos denotar por $f^{c}$ o cokernel de $f$, ou seja, $f^{c}: G_{2} \longrightarrow \frac{G_{2}}{\operatorname{Im}(f)}$.

Proposição 4.3.1. A sequência

$$
\mathbf{P i c}_{\mathcal{Z}}(R) \cap \mathbf{P i c S}_{\mathcal{Z}}(R)^{\alpha^{*}} \stackrel{\varphi_{3}}{\longrightarrow} \mathcal{C}_{0}(\Theta / R) \stackrel{\varphi_{4}}{\longrightarrow} \mathcal{B}(\Theta / R)
$$

onde $\varphi_{4}$ é $\mathcal{L}^{c}$ restrito a $\mathcal{C}_{0}(\Theta / R)$, é exata.

Demonstração. Claramente $\operatorname{Im}\left(\varphi_{3}\right) \subseteq \operatorname{ker}\left(\varphi_{4}\right)$. Seja $[\Delta(\Gamma)] \in \mathcal{C}_{0}(\Theta / R)$, tal que $\varphi_{4}([\Delta(\Gamma)])=[1]$ em $\mathcal{B}(\Theta / R)$. Então existe $[P] \in \mathbf{P i c}_{\mathcal{Z}}(R)^{(\bar{G})}$ tal que $\mathcal{L}([P])=[\Delta(\Gamma)]$. Em particular, $\Gamma_{x} \simeq P \otimes \Theta_{x} \otimes P^{-1}$, para todo $x \in G$. Assim, temos

$$
\Theta_{x} \simeq \Gamma_{x} \simeq P \otimes \Theta_{x} \otimes P^{-1} \text {, para todo } x \in G .
$$

Então, $\Theta_{x} \otimes P \simeq P \otimes \Theta_{x}$, para todo $x \in G$. Logo, $[P] \in \operatorname{Pic}_{\mathcal{Z}}(R) \cap \operatorname{PicS}_{\mathcal{Z}}(R)^{\alpha^{*}}$. Portanto, $[\Delta(\Gamma)] \in$ $\operatorname{Im}\left(\varphi_{3}\right)$.

\subsection{O grupo $\overline{H^{1}}\left(G, \alpha^{*}, \operatorname{PicS}_{0}(R)\right)$ e a quarta sequência exata}

Defina

$$
\mathbf{P i c S}_{0}(R)=\{[P] \in \mathbf{P i c S}(R) ; P \mid R \text { como bimódulos }\}
$$


Lema 4.4.1. (i) $\operatorname{PicS}_{0}(R)$ é comutativo.

(ii) $\operatorname{PicS}_{0}(R) \subseteq \operatorname{PicS}_{\mathcal{Z}}(R)$.

(iii) Se $[P] \in \mathbf{P i c S}_{0}(R)$, então $\left[\Theta_{x} \otimes P \otimes \Theta_{x^{-1}}\right] \in \mathbf{P i c S}_{0}(R)$.

(iv) $\mathcal{U}\left(\mathbf{P i c S}_{0}(R)\right) \subseteq \mathbf{P i c}_{\mathcal{Z}}(R)^{(G)}$.

Demonstração. (i) Segue diretamente da Proposição 2.3.5.

(ii) Seja $[P] \in \mathbf{P i c S}_{0}(R)$, pela Observação 2.3.3 temos que $P$ é um $\mathcal{Z}$-bimódulo central. Portanto, $\operatorname{PicS}_{0}(R) \subseteq \operatorname{PicS}_{\mathcal{Z}}(R)$.

(iii) Se $[P] \in \mathbf{P i c S}_{0}(R)$, então $P \mid R$ e pela compatibilidade com o produto tensorial, $\Theta_{x} \otimes P \otimes$ $\Theta_{x^{-1}} \mid \Theta_{x} \otimes \Theta_{x^{-1}} \simeq R 1_{x}$. Segue então pela transitividade que $\Theta_{x} \otimes P \otimes \Theta_{x^{-1}} \mid R$, para todo $x \in G$. Portanto, $\left[\Theta_{x} \otimes P \otimes \Theta_{x^{-1}}\right] \in \mathbf{P i c S}_{0}(R)$.

(iv) Se $[P] \in \mathcal{U}\left(\mathbf{P i c S}_{0}(R)\right)$, então $[P] \in \mathbf{P i c}_{\mathcal{Z}}(R)$. Como $P \mid R$ e $P^{-1} \mid R$, então $P \otimes \Theta_{x} \otimes P^{-1} \mid \Theta_{x}$, para todo $x \in G$. Logo, $[P] \in \operatorname{Pic}_{\mathcal{Z}}(R)^{(G)}$.

Segue dos itens (ii) e (iii) do Lema 4.4.1 que podemos restringir os isomorfismos $\alpha_{x}^{*}: \mathbf{P i c S}_{\mathcal{Z}}(R)\left[R 1_{x^{-1}}\right] \longrightarrow$ $\operatorname{PicS}_{\mathcal{Z}}(R)\left[R 1_{x}\right]$, definido por $\alpha_{x}^{*}\left([P]\left[R 1_{x}\right]\right)=\left[\Theta_{x} \otimes P \otimes \Theta_{x^{-1}}\right]$, para os ideais $\mathbf{P i c} \mathbf{S}_{0}(R)\left[R 1_{x^{-1}}\right]$ e temos uma ação parcial de $G$ sobre $\mathbf{P i c S}_{0}(R)$.

Lema 4.4.2. A aplicação

$$
\begin{aligned}
\zeta: \mathcal{C}(\Theta / R) & \longrightarrow Z^{1}\left(G, \alpha^{*}, \mathbf{P i c S}_{0}(R)\right) \\
{[\Delta(\Gamma)] } & \longmapsto
\end{aligned}
$$

é um morfismo de grupos cujo núcleo é $\mathcal{C}_{0}(\Theta / R)$.

Demonstração. Dado $[\Delta(\Gamma)] \in \mathcal{C}(\Theta / R)$, vamos denotar $\zeta([\Delta(\Gamma)])=f^{\Gamma}$. Então $f^{\Gamma}(x)=\left[\Gamma_{x} \otimes \Theta_{x^{-1}}\right]$, para todo $x \in G$. Como $\Gamma_{x} \mid \Theta_{x}$, então $\Gamma_{x} \otimes \Theta_{x^{-1}} \mid R$, para todo $x \in G$. Assim, $\left[\Gamma_{x} \otimes \Theta_{x^{-1}}\right] \in \operatorname{PicS}_{0}(R)$. Além disso, temos $\left[\Theta_{x} \otimes_{R} \Gamma_{x^{-1}}\right] \in \mathbf{P i c S}_{0}(R)\left[R 1_{x}\right] \mathrm{e}$

$$
\begin{aligned}
{\left[\Gamma_{x} \otimes \Theta_{x^{-1}}\right]\left[\Theta_{x} \otimes \Gamma_{x^{-1}}\right] } & =\left[\Gamma_{x} \otimes \Theta_{x^{-1}} \otimes \Theta_{x} \otimes \Gamma_{x^{-1}}\right] \\
& =\left[\Gamma_{x} \otimes R 1_{x^{-1}} \otimes \Gamma_{x^{-1}}\right] \\
& =\left[\Gamma_{x} \otimes \Gamma_{x^{-1}}\right]=\left[R 1_{x}\right] .
\end{aligned}
$$

Logo, $f^{\Gamma}(x)=\left[\Gamma_{x} \otimes_{R} \Theta_{x^{-1}}\right] \in \mathcal{U}\left(\mathbf{P i c S}_{0}(R)\left[R 1_{x}\right]\right)$, para todo $x \in G$. Dados $x, y \in G$, por Lema 3.2.1 item (iii) temos

$$
\begin{aligned}
\alpha_{x}^{*}\left(f^{\Gamma}(y)\left[R 1_{x^{-1}}\right]\right) f^{\Gamma}(x y)^{-1} f^{\Gamma}(x) & =\left[\Theta_{x} \otimes \Gamma_{y} \otimes \Theta_{y^{-1}} \otimes \Theta_{x^{-1}}\right]\left[\Theta_{x y} \otimes \Gamma_{(x y)^{-1}}\right]\left[\Gamma_{x} \otimes \Theta_{x^{-1}}\right] \\
& =\left[\Theta_{x} \otimes \Gamma_{y} \otimes \Theta_{y^{-1}} \otimes \Theta_{x^{-1}} \otimes \Theta_{x y} \otimes \Gamma_{(x y)^{-1}} \otimes \Gamma_{x} \otimes \Theta_{x^{-1}}\right] \\
& =\left[\Theta_{x} \otimes \Gamma_{y} \otimes R 1_{y^{-1}} \otimes R 1_{(x y)^{-1}} \otimes \Gamma_{(x y)^{-1}} \otimes \Gamma_{y^{-1}} \otimes \Theta_{x^{-1}}\right] \\
& =\left[\Theta_{x} \otimes \Gamma_{y} \otimes \Gamma_{(x y)^{-1}} \otimes \Gamma_{y^{-1}} \otimes \Theta_{x^{-1}}\right] \\
& =\left[\Theta_{x} \otimes R 1_{y} \otimes R 1_{x^{-1}} \otimes \Theta_{x^{-1}}\right] \\
& =\left[R 1_{x y} \otimes \Theta_{x} \otimes \Theta_{x^{-1}}\right] \\
& =\left[R 1_{x y} \otimes R 1_{x}\right]=\left[R 1_{x}\right]\left[R 1_{x y}\right] .
\end{aligned}
$$

Logo, $f^{\Gamma} \in Z^{1}\left(G, \alpha^{*}, \mathbf{P i c S}_{0}(R)\right)$, para todo $[\Delta(\Gamma)] \in \mathcal{C}(\Theta / R)$. 
Se $[\Delta(\Gamma)]=[\Delta(\Omega)]$ em $\mathcal{C}(\Theta / R)$, então $\Gamma_{x} \simeq \Omega_{x}$, para todo $x \in G$. Então, $\Gamma_{x} \otimes \Theta_{x^{-1}} \simeq \Omega_{x} \otimes \Theta_{x^{-1}}$, para todo $x \in G$. Logo,

$$
f^{\Gamma}(x)=\left[\Gamma_{x} \otimes \Theta_{x^{-1}}\right]=\left[\Omega_{x} \otimes \Theta_{x^{-1}}\right]=f^{\Omega}(x), \text { para todo } x \in G .
$$

Assim, $f^{\Gamma}=f^{\Omega}$ em $Z^{1}\left(G, \alpha^{*}, \mathbf{P i c S}_{0}(R)\right)$ e portanto $\zeta$ está bem definida.

$\operatorname{Sejam}[\Delta(\Gamma)],[\Delta(\Omega)] \in \mathcal{C}(\Theta / R)$. Como $[\Delta(\Gamma)][\Delta(\Omega)]=\left[\bigoplus_{x \in G} \Gamma_{x} \otimes \Theta_{x^{-1}} \otimes \Omega_{x}\right]$ em $\mathcal{C}(\Theta / R)$, então

$$
\begin{aligned}
f^{\Gamma \Omega}(x) & =\left[\Gamma_{x} \otimes \Theta_{x^{-1}} \otimes \Omega_{x} \otimes \Theta_{x^{-1}}\right] \\
& =\left[\Gamma_{x} \otimes \Theta_{x^{-1}}\right]\left[\Omega_{x} \otimes \Theta_{x^{-1}}\right]=f^{\Gamma}(x) f^{\Omega}(x),
\end{aligned}
$$

para todo $x \in G$. Logo, $f^{\Gamma \Omega}=f^{\Gamma} f^{\Omega}$ em $Z^{1}\left(G, \alpha^{*}, \mathbf{P i c S}_{0}(R)\right)$. Portanto $\zeta$ é morfismo de grupos.

Resta verificar que $\operatorname{ker}(\zeta)=\mathcal{C}_{0}(\Theta / R)$. Seja $[\Delta(\Gamma)] \in \mathcal{C}_{0}(\Theta / R)$, então $\Gamma_{x} \simeq \Theta_{x}$, para todo $x \in G$. Assim,

$$
f^{\Gamma}(x)=\left[\Gamma_{x} \otimes \Theta_{x^{-1}}\right]=\left[\Theta_{x} \otimes \Theta_{x^{-1}}\right]=\left[R 1_{x}\right], \text { para todo } x \in G .
$$

Logo, $[\Delta(\Gamma)] \in \operatorname{ker}(\zeta)$. Por outro lado, seja $[\Delta(\Gamma)] \in \operatorname{ker}(\zeta)$. Então, $\Gamma_{x} \otimes \Theta_{x^{-1}} \simeq R 1_{x}$, para todo $x \in G$. Fazendo o produto tensorial com $\Theta_{x}$, temos

$$
\Gamma_{x} \otimes R 1_{x^{-1}} \simeq R 1_{x} \otimes \Theta_{x} \Rightarrow \Gamma_{x} \simeq \Theta_{x}, \text { para todo } x \in G .
$$

Assim, $[\Delta(\Gamma)] \in \mathcal{C}_{0}(\Theta / R)$.

Definimos o grupo $\overline{H^{1}}\left(G, \alpha^{*}, \mathbf{P i c S}_{0}(R)\right)$ pela sequência exata

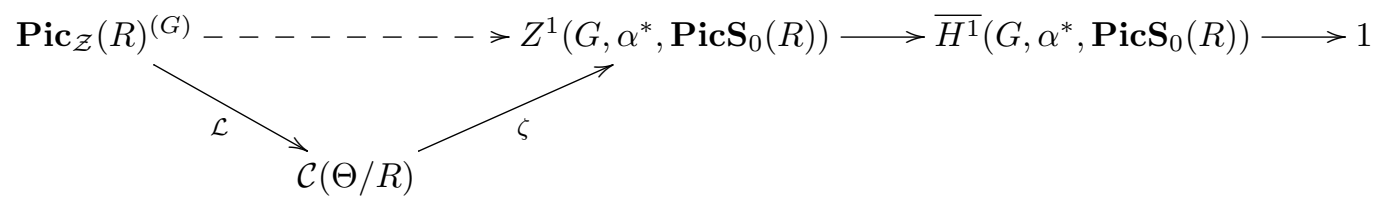

ou seja,

$$
\overline{H^{1}}\left(G, \alpha^{*}, \mathbf{P i c S}_{0}(R)\right)=\frac{Z^{1}\left(G, \alpha^{*}, \mathbf{P i c S}_{0}(R)\right)}{(\zeta \circ \mathcal{L})\left(\mathbf{P i c}_{\mathcal{Z}}(R)^{(G)}\right)} .
$$

Observação 4.4.3. Uma pergunta que surge naturalmente é: Qual a relação entre $B^{1}\left(G, \alpha^{*}, \mathbf{P i c S}_{0}(R)\right)$ e a imagem de $\zeta \circ \mathcal{L}$ ? A inclusão $B^{1}\left(G, \alpha^{*}, \mathbf{P i c S}_{0}(R)\right) \subseteq \operatorname{Im}(\zeta \circ \mathcal{L})$ é sempre verdadeira. De fato, seja $\rho \in B^{1}\left(G, \alpha^{*}, \mathbf{P i c S}_{0}(R)\right)$, então existe $[P] \in \mathcal{U}\left(\mathbf{P i c S}_{0}(R)\right) \subseteq \mathbf{P i c}_{\mathcal{Z}}(R)^{(G)}$ (ver Lema 4.4.1), tal que

$$
\begin{aligned}
\rho(x) & =\alpha_{x}^{*}\left([P]\left[R 1_{x^{-1}}\right]\right)\left[P^{-1}\right]=\left[\Theta_{x} \otimes P \otimes \Theta_{x^{-1}}\right]\left[P^{-1}\right] \\
& =\left[\Theta_{x} \otimes P \otimes \Theta_{x^{-1}} \otimes P^{-1}\right]=\left[P^{-1} \otimes \Theta_{x} \otimes P \otimes \Theta_{x^{-1}}\right] \\
& =\zeta\left(\mathcal{L}\left(\left[P^{-1}\right]\right)\right)(x),
\end{aligned}
$$

para todo $x \in G$. Logo, $B^{1}\left(G, \alpha^{*}, \mathbf{P i c S}_{0}(R)\right) \subseteq \operatorname{Im}(\zeta \circ \mathcal{L})$.

Vamos definir o morfismo $\varphi_{5}: \mathcal{B}(\Theta / R) \longrightarrow \overline{H^{1}}\left(G, \alpha^{*}, \mathbf{P i c}_{0}(R)\right)$ através do diagrama comutativo

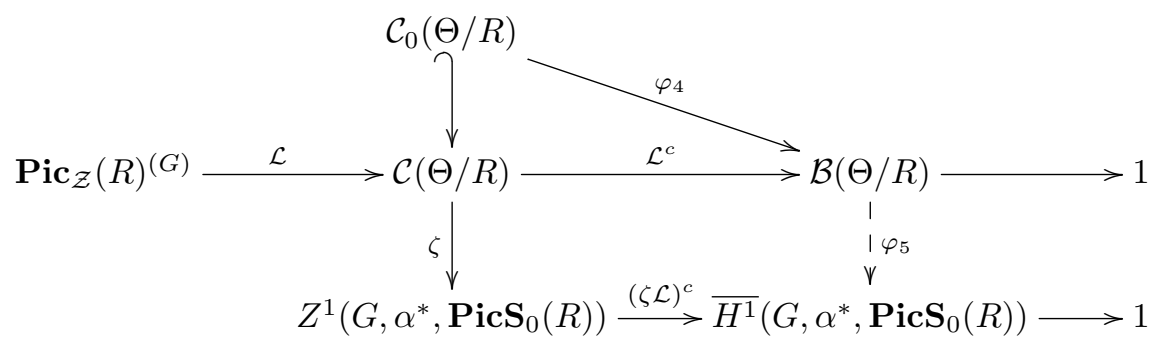


Observação 4.4.4. $\varphi_{5}$ está bem definida.

De fato, sejam $[\Delta(\Gamma)]$ e $[\Delta(\Omega)]$ em $\mathcal{C}(\Theta / R)$, tais que $\mathcal{L}^{c}([\Delta(\Gamma)])=\mathcal{L}^{c}([\Delta(\Omega)])$ em $\mathcal{B}(\Theta / R)$. Então, existe $[P] \in \mathbf{P i c}_{\mathcal{Z}}(R)^{(G)}$, com $[\Delta(\Gamma)]=[\Delta(\Omega)] \mathcal{L}([P])$. Pela comutatividade do diagrama, temos

$$
\begin{aligned}
\varphi_{5}\left(\mathcal{L}^{c}([\Delta(\Gamma)])\right) & =\left((\zeta \mathcal{L})^{c} \circ \zeta\right)([\Delta(\Gamma)]) \\
& =\left((\zeta \mathcal{L})^{c} \circ \zeta\right)([\Delta(\Omega)] \mathcal{L}([P])) \\
& =(\zeta \mathcal{L})^{c}(\zeta([\Delta(\Omega)] \zeta \mathcal{L}([P])) \\
& =(\zeta \mathcal{L})^{c}(\zeta([\Delta(\Omega)]) \\
& =\varphi_{5}\left(\mathcal{L}^{c}([\Delta(\Omega)])\right) .
\end{aligned}
$$

Portanto, $\varphi_{5}$ não depende da escolha do representante da classe em $\mathcal{B}(\Theta / R)$.

Proposição 4.4.5. A sequência

$$
\mathcal{C}_{0}(\Theta / R) \stackrel{\varphi_{4}}{\longrightarrow} \mathcal{B}(\Theta / R) \stackrel{\varphi_{5}}{\longrightarrow} \overline{H^{1}}\left(G, \alpha^{*}, \mathbf{P i c S}_{0}(R)\right)
$$

é exata.

Demonstração. Seja $[\Delta(\Gamma)] \in \mathcal{C}_{0}(\Theta / R)$. Pela comutatividade do diagrama temos que $\varphi_{5}\left(\varphi_{4}([\Delta(\Gamma)])\right)=$ $\left((\zeta \mathcal{L})^{c} \circ \zeta\right)([\Delta(\Gamma)])$. Pelo Lema 4.4.2, temos que $\zeta([\Delta(\Gamma)])=[1]$. Assim, $\varphi_{5}\left(\varphi_{4}([\Delta(\Gamma)])\right)=[1]$. Logo, $\operatorname{Im}\left(\varphi_{4}\right) \subseteq \operatorname{ker}\left(\varphi_{5}\right)$.

Dado $[\Delta(\Gamma)] \in \mathcal{C}(\Theta / R)$, denote $\overline{[\Delta(\Gamma)]}=\mathcal{L}^{c}([\Delta(\Gamma)])$ em $\mathcal{B}(\Theta / R)$. Se $\varphi_{5}(\overline{[\Delta(\Gamma)]})=[1]$, pela comutatividade do diagrama, temos

$$
\left((\zeta \mathcal{L})^{c} \circ \zeta\right)([\Delta(\Gamma)])=[1] \text { em } \overline{H^{1}}\left(G, \alpha^{*}, \mathbf{P i c S}_{0}(R)\right) .
$$

Então, $\zeta([\Delta(\Gamma)]) \in(\zeta \mathcal{L})\left(\mathbf{P i c}_{\mathcal{Z}}(R)^{(G)}\right)$. Seja $[P] \in \mathbf{P i c}_{\mathcal{Z}}(R)^{(G)}$, tal que $\zeta([\Delta(\Gamma)])=\zeta(\mathcal{L}([P]))$. Como $\zeta$ é morfismo de grupos, segue que $[\Delta(\Gamma)] \mathcal{L}[P]^{-1} \in \operatorname{ker}(\zeta)=\mathcal{C}_{0}(\Theta / R)$. Então,

$$
\begin{aligned}
\varphi_{4}\left([\Delta(\Gamma)] \mathcal{L}([P])^{-1}\right) & =\varphi_{4}\left([\Delta(\Gamma)] \mathcal{L}\left(\left[P^{-1}\right]\right)\right) \\
& =\mathcal{L}^{c}([\Delta(\Gamma)]) \mathcal{L}^{c}\left(\mathcal{L}\left(\left[P^{-1}\right]\right)\right) \\
& =\mathcal{L}^{c}([\Delta(\Gamma)])=\overline{[\Delta(\Gamma)]}
\end{aligned}
$$

Logo, $\operatorname{ker}\left(\varphi_{5}\right) \subseteq \operatorname{Im}\left(\varphi_{4}\right)$. Portanto, a sequência é exata.

\subsection{A quinta sequência exata}

Seja $g$ um 1-cociclo normalizado em $Z^{1}\left(G, \alpha^{*}, \mathbf{P i c S}_{0}(R)\right)$, ou seja,

$$
\begin{aligned}
g: G & \longrightarrow \operatorname{PicS}_{0}(R) \\
x & \longrightarrow\left[\nabla_{x}\right]
\end{aligned}
$$

onde $\left[\nabla_{x}\right] \in \mathcal{U}\left(\mathbf{P i c S}_{0}(R)\left[R 1_{x}\right]\right)$, para todo $x \in G$ e

$$
\alpha_{x}^{*}\left(g_{y}\left[R 1_{x^{-1}}\right]\right) g_{x y}^{-1} g_{x}=\left[R 1_{x}\right]\left[R 1_{x y}\right], \quad \text { para todo } x, y \in G .
$$

Observe que temos

$$
\alpha_{x}^{*}\left(g_{y}\left[R 1_{x^{-1}}\right]\right)\left[\Theta_{x}\right]=\left[\Theta_{x}\right] g_{y}, \text { para todo } x, y \in G
$$


De fato,

$$
\begin{aligned}
\alpha_{x}^{*}\left(g_{y}\left[R 1_{x^{-1}}\right]\right)\left[\Theta_{x}\right] & =\left[\Theta_{x} \otimes \nabla_{y} \otimes \Theta_{x^{-1}}\right]\left[\Theta_{x}\right] \\
& =\left[\Theta_{x} \otimes \nabla_{y} \otimes R 1_{x^{-1}}\right] \\
& =\left[\Theta_{x} \otimes R 1_{x^{-1}} \otimes \nabla_{y}\right] \\
& =\left[\Theta_{x} \otimes \nabla_{y}\right] \\
& =\left[\Theta_{x}\right] g_{y} .
\end{aligned}
$$

Lema 4.5.1. Seja $g$ um elemento normalizado em $Z^{1}\left(G, \alpha^{*}, \mathbf{P i c}_{0}(R)\right)$. Então,

$$
\begin{aligned}
U: G & \longrightarrow \operatorname{PicS}(R) \\
x & \longmapsto g_{x}\left[\Theta_{x}\right]
\end{aligned}
$$

é uma representação parcial unital com $U_{x} \otimes U_{x^{-1}} \simeq R 1_{x}$ e $U_{x} \mid \Theta_{x}$, para todo $x \in G$.

Demonstração. Como $g$ é normalizado, então $\left[U_{1}\right]=g_{1}\left[\Theta_{1}\right]=[R]$. Dados $x, y \in G$ temos

$$
\begin{aligned}
& {\left[U_{x}\right]\left[U_{y}\right]\left[U_{y^{-1}}\right]=g_{x}\left[\Theta_{x}\right] g_{y}\left[\Theta_{y}\right] g_{y^{-1}}\left[\Theta_{y^{-1}}\right]} \\
& \stackrel{(4.14)}{=} g_{x} \alpha_{x}^{*}\left(g_{y}\left[R 1_{x^{-1}}\right]\right)\left[\Theta_{x}\right]\left[\Theta_{y}\right] g_{y^{-1}}\left[\Theta_{y^{-1}}\right] \\
& \stackrel{(4.13)}{=} g_{x y}\left[R 1_{x}\right]\left[R 1_{x}\right]\left[\Theta_{x y}\right] g_{y^{-1}}\left[\Theta_{y^{-1}}\right] \\
& =g_{x y}\left[R 1_{x}\right]\left[\Theta_{x y}\right] g_{y^{-1}}\left[\Theta_{y^{-1}}\right] \\
& =g_{x y}\left[\Theta_{x y}\right]\left[R 1_{y^{-1}}\right] g_{y^{-1}}\left[\Theta_{y^{-1}}\right] \\
& =g_{x y}\left[\Theta_{x y}\right] g_{y^{-1}}\left[R 1_{y^{-1}}\right]\left[\Theta_{y^{-1}}\right] \\
& =g_{x y}\left[\Theta_{x y}\right] g_{y^{-1}}\left[\Theta_{y^{-1}}\right] \\
& =\left[U_{x y}\right]\left[U_{y^{-1}}\right] \text {. }
\end{aligned}
$$

Analogamente,

$$
\begin{aligned}
& {\left[U_{x^{-1}}\right]\left[U_{x}\right]\left[U_{y}\right]=g_{x^{-1}}\left[\Theta_{x^{-1}}\right] g_{x}\left[\Theta_{x}\right] g_{y}\left[\Theta_{y}\right]} \\
& \stackrel{(4.14)}{=} g_{x^{-1}}\left[\Theta_{x^{-1}}\right] g_{x} \alpha_{x}^{*}\left(g_{y}\left[R 1_{x^{-1}}\right]\right)\left[\Theta_{x}\right]\left[\Theta_{y}\right] \\
& \stackrel{(4.13)}{=} g_{x^{-1}}\left[\Theta_{x^{-1}}\right] g_{x y}\left[R 1_{x}\right]\left[R 1_{x}\right]\left[\Theta_{x y}\right] \\
& =g_{x^{-1}}\left[\Theta_{x^{-1}}\right] g_{x y}\left[R 1_{x}\right]\left[\Theta_{x y}\right] \\
& =g_{x^{-1}}\left[\Theta_{x^{-1}}\right]\left[R 1_{x}\right] g_{x y}\left[\Theta_{x y}\right] \\
& =g_{x^{-1}}\left[\Theta_{x^{-1}}\right] g_{x y}\left[\Theta_{x y}\right] \\
& =\left[U_{x^{-1}}\right]\left[U_{x y}\right],
\end{aligned}
$$

para todo $x, y \in G$. Novamente por $g$ ser normalizado temos:

$$
\begin{array}{rll}
{\left[U_{x}\right]\left[U_{x^{-1}}\right]} & \stackrel{(4.14)}{=} g_{x}\left[\Theta_{x}\right] g_{x^{-1}}\left[\Theta_{x^{-1}}\right] \\
& \stackrel{\left({ }_{x} \alpha_{x}^{*}\left(g_{x^{-1}}\left[R 1_{x^{-1}}\right]\right)\left[\Theta_{x}\right]\left[\Theta_{x^{-1}}\right]\right.}{=} \quad\left[g_{1}\right]\left[R 1_{x}\right]=[R]\left[R 1_{x}\right]=\left[R 1_{x}\right],
\end{array}
$$

para todo $x \in G$. Além disso, como $g_{x}=\left[\nabla_{x}\right] \in \mathbf{P i c S}_{0}(R)$, então $\nabla_{x} \mid R$, para todo $x \in G$. Assim $U_{x} \simeq \nabla_{x} \otimes \Theta_{x} \mid \Theta_{x}$, para todo $x \in G$.

Por $U$ ser representação parcial unital segue do Lema 3.2.1 que existe uma família de isomorfismos de $R$-bimódulos

$$
f^{g}=\left\{f_{x, y}^{g}: U_{x} \otimes_{R} U_{y} \longrightarrow R 1_{x} \otimes U_{x y} \simeq 1_{x} U_{x y}, \forall x, y \in G\right\}
$$


Como $\left[U_{1}\right]=[R]$, podemos escolher $\left\{U_{x}\right\}_{x \in G}$ uma família de representantes com $U_{1}=R$ e $f^{g}$ uma família de isomorfismos de $R$-bimódulos com $f_{x, 1}^{g}: U_{x} \otimes R \longrightarrow U_{x}$ e $f_{1, x}^{g}: R \otimes U_{x} \longrightarrow U_{x}$ dados pela ação de $R$ sobre $U_{x}$ à direita e à esquerda, respectivamente. Então, os diagramas comutativos em (3.23) são trivialmente satisfeitos. Logo, pelo Corolário 3.3.6, existe um 3-cociclo parcial $\widetilde{\beta_{-,-,-}^{g}}$ em $Z_{\Theta}^{3}(G, \alpha, \mathcal{Z})$ associado a $f^{g}$. Pela Proposição 3.3.7, $\left[\widetilde{\beta_{-,-,-}^{g}}\right]$ em $H_{\Theta}^{3}(G, \alpha, \mathcal{Z})$ não depende da escolha da família de $R$-bimódulos $\left\{U_{x}\right\}_{x \in G}$ e nem da escolha da família de isomorfismos de $R$-bimódulos $f^{g}$, então temos uma aplicação bem definido:

Lema 4.5.2. A aplicação

$$
\begin{array}{cl}
\delta: Z^{1}\left(G, \alpha^{*}, \mathbf{P i c S}_{0}(R)\right) & \longrightarrow H_{\Theta}^{3}(G, \alpha, \mathcal{Z}) \\
g & \longmapsto\left[\beta_{-,-,-}^{g}\right]
\end{array}
$$

é um morfismo de grupos. Além disso,

$$
\delta \circ \zeta=[1]
$$

onde $\zeta$ é o morfismo definido no Lema 4.4.2.

Demonstração. Sejam $g, h \in Z^{1}\left(G, \alpha^{*}, \mathbf{P i c S}_{0}(R)\right)$. Denote $\left[U_{x}\right]=g_{x}\left[\Theta_{x}\right]$ e $\left[V_{x}\right]=h_{x}\left[\Theta_{x}\right]$, para todo $x \in G$. Considere

$$
f_{x, y}^{g}: U_{x} \otimes U_{y} \longrightarrow 1_{x} U_{x y} \text { e } f_{x, y}^{h}: V_{x} \otimes V_{y} \longrightarrow 1_{x} V_{x, y} \quad x, y \in G
$$

famílias de isomorfismos de $R$-bimódulos e $\widetilde{\beta_{-,-,-}^{g}}$ e $\widetilde{\beta_{-,-,-}^{h}}$ os 3-cociclos em $Z_{\Theta}^{3}(G, \alpha, \mathcal{Z})$ associados. Seja $\left[W_{x}\right]=g_{x} h_{x}\left[\Theta_{x}\right]$, para todo $x \in G$. Então,

$$
\begin{aligned}
{\left[W_{x}\right] } & =g_{x} h_{x}\left[\Theta_{x}\right]=g_{x} h_{x}\left[R 1_{x}\right]\left[\Theta_{x}\right] \\
& =g_{x}\left[R 1_{x}\right] h_{x}\left[\Theta_{x}\right]=g_{x}\left[\Theta_{x}\right]\left[\Theta_{x^{-1}}\right] h_{x}\left[\Theta_{x}\right] \\
& =\left[U_{x} \otimes \Theta_{x^{-1}} \otimes V_{x}\right],
\end{aligned}
$$

para todo $x \in G$. Considere os isomorfismos de $R$-bimódulos $f_{x, y}^{g h}$ definidos por

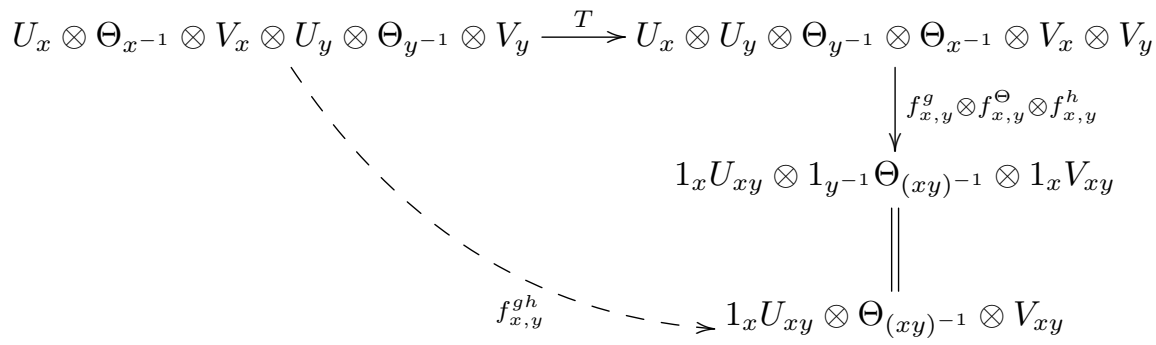

onde $T: \Theta_{x^{-1}} \otimes V_{x} \otimes U_{y} \otimes \Theta_{y^{-1}} \longrightarrow U_{y} \otimes \Theta_{y^{-1}} \otimes \Theta_{x^{-1}} \otimes V_{x}$ é o isomorfismo da Proposição 2.3.5. Pelo Lema 3.3.8, temos que $\widetilde{\beta_{-,-,-}^{g h}}=\widetilde{\beta_{-,-,-}^{g}} \widetilde{\beta_{-,-,-}^{h}}$. Logo,

$$
\left.\delta(g h)=\left[\widetilde{\beta_{-,-,-}^{g h}}\right]=\left[\widetilde{\beta_{-,-,-}^{g}} \widetilde{\beta_{-,-,-}^{h}}\right]=\left[\widetilde{\beta_{-,-,-}^{g}}\right] \widetilde{\beta_{-,-,-}^{h}}\right]=\delta(g) \delta(h) .
$$

e portanto $\delta$ é um morfismo de grupos. Para a ultima afirmação, seja $[\Delta(\Gamma)] \in \mathcal{C}(\Theta / R)$ e denote $f^{\Gamma}=\zeta([\Delta(\Gamma)])$, ou seja, $f_{x}^{\Gamma}=\left[\Gamma_{x}\right]\left[\Theta_{x^{-1}}\right]$, para todo $x \in G$. Assim,

$$
\left[U_{x}\right]:=f_{x}^{\Gamma}\left[\Theta_{x}\right]=\left[\Gamma_{x}\right]\left[\Theta_{x^{-1}}\right]\left[\Theta_{x}\right]=\left[\Gamma_{x}\right]\left[R 1_{x^{-1}}\right]=\left[\Gamma_{x}\right], \text { para todo } x \in G .
$$

Considerando a família de isomorfismos de $R$-bimódulos $f^{\Gamma}=\left\{f_{x, y}^{\Gamma}: \Gamma_{x} \otimes \Gamma_{y} \longrightarrow 1_{x} \Gamma_{x y}, x, y \in G\right\}$ que é um conjunto de fatores para $\Delta(\Gamma)$. Então, $\widetilde{\beta_{-,-,-}^{f^{\Gamma}}}$ é trivial. Logo, $\delta(\zeta([\Delta(\Gamma)]))=[1] \operatorname{em~} H_{\Theta}^{3}(G, \alpha, \mathcal{Z})$. 
Vamos definir $\varphi_{6}: \overline{H^{1}}\left(G, \alpha^{*}, \mathbf{P i c S}_{0}(R)\right) \longrightarrow H_{\Theta}^{3}(G, \alpha, \mathcal{Z})$ via o diagrama comutativo

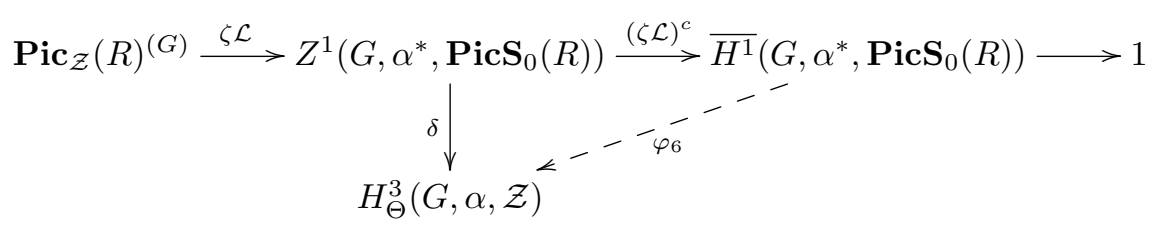

Vejamos que $\varphi_{6}$ está bem definida: Sejam $h, g \in Z^{1}\left(G, \alpha^{*}, \mathbf{P i c S}_{0}(R)\right)$ tais que $[h]=[g] \operatorname{em} \overline{H^{1}}\left(G, \alpha^{*}, \mathbf{P i c S}_{0}(R)\right)$, então existe $[P] \in \mathbf{P i c}_{\mathcal{Z}}(R)^{(G)}$ tal que $h=g \zeta(\mathcal{L}([P]))$. Por $(4.16)$, temos que

$$
\delta(\zeta(\mathcal{L}([P])))=(\delta \circ \zeta)(\mathcal{L}([P]))=[1] \text { em } H_{\Theta}^{3}(G, \alpha, \mathcal{Z}) .
$$

Pela comutatividade do diagrama, temos

$$
\begin{aligned}
\varphi_{6}([h]) & =\left(\varphi_{6} \circ(\zeta \mathcal{L})^{c}\right)(h) \\
& =\delta(h)=\delta(g \zeta(\mathcal{L}([P]))) \\
& =\delta(g) \delta(\zeta(\mathcal{L}([P]))) \\
& =\delta(g)=\left(\varphi_{6} \circ(\zeta \mathcal{L})^{c}\right)(g) \\
& =\varphi_{6}([g]) .
\end{aligned}
$$

Portanto, $\varphi_{6}$ está bem definida.

Assim, temos o seguinte diagrama comutativo

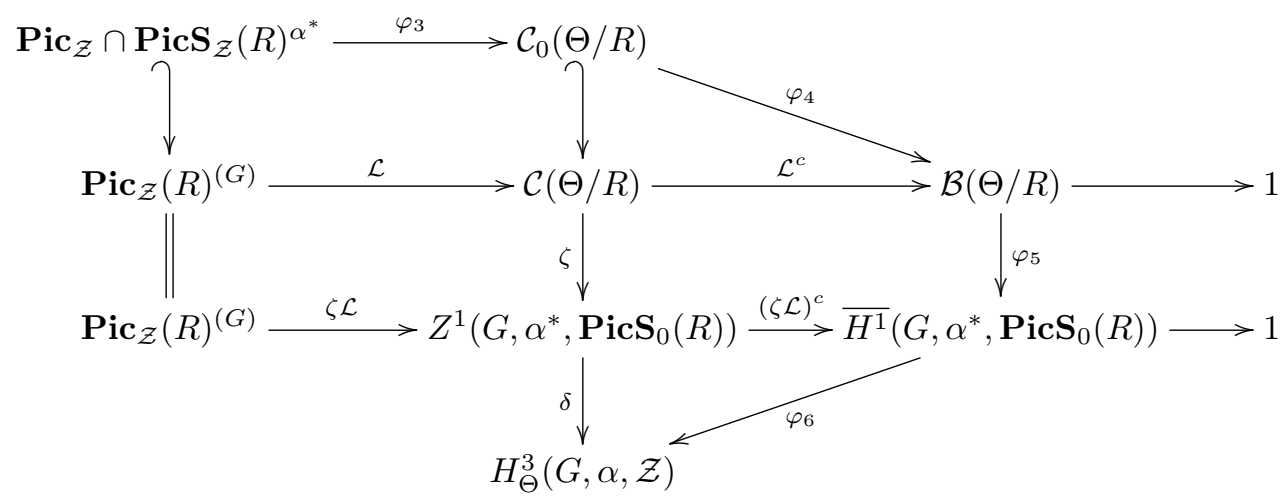

Proposição 4.5.3. A sequência

$$
\mathcal{B}(\Theta / R) \stackrel{\varphi_{5}}{\longrightarrow} \overline{H^{1}}\left(G, \alpha^{*}, \mathbf{P i c S}_{0}(R)\right) \stackrel{\varphi_{6}}{\longrightarrow} H_{\Theta}^{3}(G, \alpha, \mathcal{Z})
$$

é exata.

Demonstração. Seja $[h] \in \overline{H^{1}}\left(G, \alpha^{*}, \mathbf{P i c S}_{0}(R)\right)$, tal que existe $[\Delta(\Gamma)] \in \mathcal{C}_{0}(\Theta / R), \operatorname{com} \varphi_{5}\left(\mathcal{L}^{c}([\Delta(\Gamma)])\right)=$ $[h]$. Pela comutatividade do diagrama e por (4.16) temos

$$
\varphi_{6}([h])=\varphi_{6}\left(\varphi_{5}\left(\mathcal{L}^{c}([\Delta(\Gamma)])\right)\right)=(\delta \circ \zeta)([\Delta(\Gamma)])=[1] .
$$

Logo, $\operatorname{Im}\left(\varphi_{5}\right) \subseteq \operatorname{ker}\left(\varphi_{6}\right)$.

Seja $[h] \in \overline{H^{1}}\left(G, \alpha^{*}, \mathbf{P i c S}_{0}(R)\right)$ tal que $\varphi_{6}([h])=[1]$. Pela comutatividade do diagrama temos que $\delta(h)=[1]$ em $H_{\Theta}^{3}(G, \alpha, \mathcal{Z})$. Denotando $\delta(h)=\left[\beta_{-,-,-}\right] \in B_{\Theta}^{3}(G, \alpha, \mathcal{Z})$, então existe $\sigma_{-,-}: G \times G \longrightarrow \mathcal{Z}$, com $\sigma_{x, y} \in \mathcal{U}\left(\mathcal{Z} 1_{x} 1_{x y}\right)$, para todo $x, y \in G$, e

$$
\beta_{x, y, z}=\alpha_{x}\left(\sigma_{y, z} 1_{x^{-1}}\right) \sigma_{x y, z}^{-1} \sigma_{x, y z} \sigma_{x, y}^{-1},
$$


para todo $x, y, z \in G$. Defina $\left[\Omega_{x}\right]:=h_{x}\left[\Theta_{x}\right]$, para todo $x \in G$ e considere a família de isomorfismos $f^{h}$ associada como em (4.15). Então, por definição, temos que

$$
\beta_{x, y, x} \circ f_{x, y z}^{h} \circ\left(\Omega_{x} \otimes f_{y, z}^{h}\right)=f_{x y, z}^{h} \circ\left(f_{x, y}^{h} \otimes \Omega_{z}\right),
$$

para todo $x, y, z \in G$. Pelo Lema 4.5.1, temos que $\Omega: G \longrightarrow \operatorname{PicS}(R)$ definida por $\left[\Omega_{x}\right]=h_{x}\left[\Theta_{x}\right]$ é uma representação parcial unital com $\Omega_{x} \otimes \Omega_{x^{-1}} \simeq R 1_{x}$ e $\Omega_{x} \mid \Theta_{x}$, para todo $x \in G$. Pela Proposição 3.3.5, temos que

$$
\begin{aligned}
\bar{f}_{x, y}^{h}: \Omega_{x} \otimes \Omega_{y} & \longrightarrow 1_{x} \Omega_{x y} \\
\omega_{x} \otimes \omega_{y} & \longmapsto \sigma_{x, y} f_{x, y}^{h}\left(\omega_{x} \otimes \omega_{y}\right)
\end{aligned}
$$

é um conjunto de fatores para $\Omega$. Logo, $\Delta(\Omega)$ é um produto cruzado generalizado parcial com $[\Delta(\Omega)] \in$ $\mathcal{C}(\Theta / R)$. Além disso, $\zeta([\Delta(\Omega)])=h$. Então, pela comutatividade do diagrama, temos

$$
[h]=(\zeta \mathcal{L})^{c}(h)=\left((\zeta \mathcal{L})^{c} \circ \zeta\right)([\Delta(\Omega)])=\varphi_{5}\left(\mathcal{L}^{c}([\Delta(\Omega)])\right),
$$

ou seja, $[h] \in \operatorname{Im}\left(\varphi_{5}\right)$. Logo, $\operatorname{ker}\left(\varphi_{6}\right) \subseteq \operatorname{Im}\left(\varphi_{5}\right)$ e portanto a sequência é exata.

Resumimos no seguinte diagrama comutativo os resultados deste capítulo:

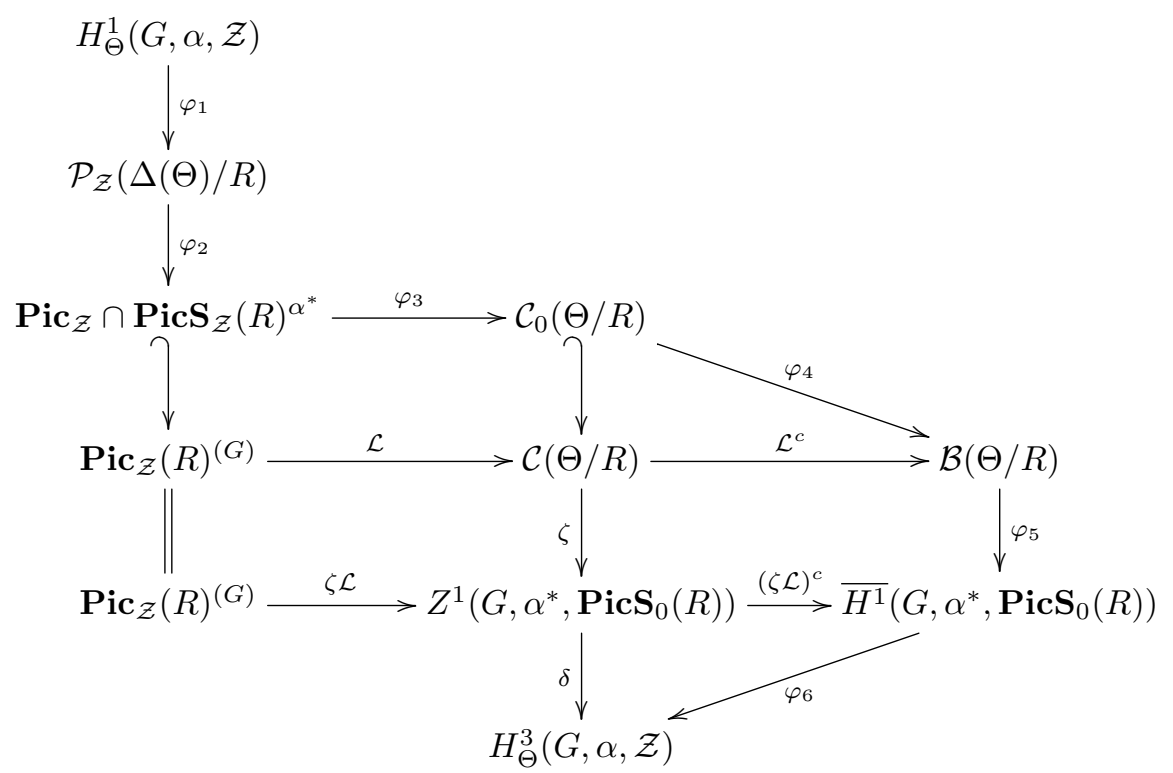

Pelo Teorema 3.4.3 temos que $\mathcal{C}_{0}(\Theta / R) \simeq H_{\Theta}^{2}(G, \alpha, \mathcal{Z})$, então pelos Teoremas 4.1.5 e 4.2 .7 e pelas Proposições 4.3.1, 4.4.5 e 4.5.3, temos o nosso resultado principal:

Teorema 4.5.4. A sequência

$$
\begin{aligned}
& 1 \longrightarrow H_{\Theta}^{1}(G, \alpha, \mathcal{Z}) \stackrel{\varphi_{1}}{\longrightarrow} \mathcal{P}_{\mathcal{Z}}(\Delta(\Theta) / R)^{(G)} \stackrel{\varphi_{2}}{\longrightarrow} \operatorname{Pic}_{\mathcal{Z}}(R) \cap \operatorname{PicS}_{\mathcal{Z}}(R)^{\alpha^{*}} \stackrel{\varphi_{3}}{\longrightarrow} H_{\Theta}^{2}(G, \alpha, \mathcal{Z}) \\
& \stackrel{\varphi_{4}}{\longrightarrow} \mathcal{B}(\Theta / R) \stackrel{\varphi_{5}}{\longrightarrow} \bar{H}^{1}\left(G, \alpha^{*}, \mathbf{P i c S}_{0}(R)\right) \stackrel{\varphi_{6}}{\longrightarrow} H_{\Theta}^{3}(G, \alpha, \mathcal{Z})
\end{aligned}
$$

é exata. 


\section{Referências Bibliográficas}

[1] M. Auslander, O. Goldman, The brauer group of a commutative ring, Transactions of the American Mathematical Society 97 (3) (1960) 367-409.

[2] S. U. Chase, D. K. Harrison, A. Rosenberg, Galois theory and cohomology of commutative rings, Vol. 52, American Mathematical Soc., 1965.

[3] S. U. Chase, A. Rosenberg, Amitsur cohomology and the Brauer group, American Mathematical Society, 1965.

[4] T. Kanzaki, On generalized crossed product and brauer group, Osaka Journal of Mathematics 5 (2) (1968) $175-188$.

[5] Y. Miyashita, An exact sequence associated with a generalized crossed product, Nagoya Mathematical Journal 49 (1973) 21-51.

[6] L. El Kaoutit, J. Gómez-Torrecillas, Invertible unital bimodules over rings with local units, and related exact sequences of groups, Journal of Algebra 323 (1) (2010) 224-240.

[7] L. El Kaoutit, J. Gómez-Torrecillas, Invertible unital bimodules over rings with local units, and related exact sequences of groups, ii, Journal of Algebra 370 (2012) 266-296.

[8] D. Crocker, I. Raeburn, D. P. Williams, Equivariant brauer and picard groups and a chase-harrisonrosenberg exact sequence, Journal of Algebra 307 (1) (2007) 397-408.

[9] R. Exel, Twisted partial actions: a classification of regular $\mathrm{c}^{*}$-algebraic bundles, Proceedings of the London Mathematical Society 74 (2) (1997) 417-443.

[10] R. Exel, Partial actions of groups and actions of inverse semigroups, Proceedings of the American Mathematical Society 126 (12) (1998) 3481-3494.

[11] F. Abadie, Sobre açoes parciais, fibrados de fell e grupóides, Ph.D. thesis, PhD Thesis, Universidade de Sao Paulo (1999).

[12] F. Abadie, Enveloping actions and takai duality for partial actions, Journal of Functional Analysis 197 (1) (2003) 14-67.

[13] M. Dokuchaev, R. Exel, Associativity of crossed products by partial actions, enveloping actions and partial representations, Transactions of the American Mathematical Society 357 (5) (2005) 19311952.

[14] M. Dokuchaev, M. Ferrero, A. Paques, Partial actions and galois theory, Journal of Pure and Applied Algebra 208 (1) (2007) 77-87.

[15] M. Dokuchaev, M. Khrypchenko, Partial cohomology of groups, Journal of Algebra 427 (2015) 142182. 
[16] M. Dokuchaev, A. Paques, H. Pinedo, Partial galois cohomology, extensions of the picard group and related homomorphisms, preprint.

[17] M. Dokuchaev, A. Paques, H. Pinedo, Partial generalized crossed products and a seven-term exact sequence, preprint.

[18] M. Yôichi, On galois extensions and crossed products, Journal of the Faculty of Science, Hokkaido University. Ser. 1, 21 (2) (1971) 97-121.

[19] F. DeMeyer, E. Ingraham, Separable algebras over commutative rings, Vol. 181, Springer, 1971.

[20] T.-Y. Lam, Lectures on modules and rings, Vol. 189, Springer Science \& Business Media, 2012.

[21] F. W. Anderson, K. R. Fuller, Rings and Categories of Modules, Vol. 13, Springer Science \& Business Media, 1992.

[22] N. Jacobson, Basic algebra I, Courier Corporation, 2012.

[23] A. Fröhlich, The picard group of noncommutative rings, in particular of orders, Transactions of the American Mathematical Society 180 (1973) 1-45.

[24] K. Morita, The endomorphism ring theorem for frobenius extensions, Mathematische Zeitschrift 102 (5) (1967) 385-404.

[25] L. El Kaoutit, Extended distributive law: cowreath over corings, Journal of Algebra and Its Applications 9 (01) (2010) 135-171.

[26] M. Dokuchaev, R. Exel, P. Piccione, Partial representations and partial group algebras, Journal of Algebra 226 (1) (2000) 505-532.

[27] M. Dokuchaev, R. Exel, J. Simón, Crossed products by twisted partial actions and graded algebras, Journal of Algebra 320 (8) (2008) 3278-3310. 